\title{
Atomic-scale insights into emergent photovoltaic absorbers
}

\author{
Alex Ganose \\ UNiversity COLLEge LONDON
}

Supervisor: Dr David Scanlon

A thesis submitted in partial fulfilment of the requirements for the degree of Doctor of Engineering at University College London.

August 27, 2018 



\section{Declaration}

I, Alexander Miguel Ganose, confirm that the work presented in this thesis is my own. Where information has been derived from other sources, I confirm that this has been indicated in the work.

Alexander Miguel Ganose

August 27, 2018

University College London 


\begin{abstract}
The world is currently experiencing an energy crisis; as energy reserves continue to be depleted at record pace, there is a growing demand for a clean and renewable energy source capable of sustaining economic growth. Arguably, solar power is the most promising renewable technology due to the enormous amount of energy that reaches the earth in the form of solar radiation. Traditional solar cells, such as those based on crystalline silicon, have achieved efficiencies up to $25 \%$ but are limited in their widespread application due to limits in their costcompetitiveness. Recently, the lead hybrid perovskites have emerged as a highly efficient class of solar absorber, with efficiencies reaching over $23 \%$ within just nine years. Unfortunately, the stability of these materials is poor and concerns over the toxicity of lead have sparked significant research effort toward the search for alternative absorbers capable of achieving comparable efficiencies.

This thesis investigates a number of materials for their suitability as solar absorbers. Throughout, $a b$ initio methods are used to provide insight into the structural, electronic, and optical properties that determine their performance. Special attention is paid to understanding the behaviour of intrinsic defects due to their critical role in determining carrier recombination and transport. Initially, perovskite-based materials are discussed, including a new family of layered perovskites, and the lead-free vacancy-ordered double perovskites. In the second part of this thesis, the search for emerging photovoltaics is extended to a promising family of bismuth-based absorbers, of interest due to their non-toxic and earth-abundant nature. Throughout this work, we aim to provide specific guidance for experimental researchers hoping to produce more efficient photovoltaic devices.
\end{abstract}




\section{Impact statement}

Climate change is currently the most pressing challenge faced by society. Photovoltaics offer some hope due to the enormous amounts of energy the sun can provide. Indeed, the potential capacity from solar technologies far exceeds that of our remaining non-renewable energy reserves. Current commercial photovoltaics are unlikely to be able to achieve the cost-competitiveness needed for large scale energy generation. There is, therefore, a strong demand both in the EU and globally, for cost-effective photovoltaics. The work presented in this thesis provides insight into novel absorber materials that may one day be used as efficienct photovoltaics at reduced cost.

In particular, this thesis examines several materials that are at the forefront of international photovoltaic research. By adopting a computational approach we are able to investigate atomic-scale properties at a level of accuracy that is difficult, if not impossible, to achieve using traditional experimental techniques. Throughout, we provide insight into the fundamental optoelectronic properties that determine photovoltaic performance. Much attention is given toward providing specific advice to experimental colleagues, with the aim of enabling more efficient devices. In this way, this work can increase the pace of photovoltaic progress by directing the focus of future research. In addition, while in this thesis our results have been interpreted in the context of photovoltaics, this work may be of interest for multiple practical applications. This research has been disseminated through both research publications and conference presentations, including 14 published manuscripts relating directly to the thesis topic. 


\section{Acknowledgements}

I owe a huge debt of gratitude to my supervisor Dr David Scanlon — initially for taking the risk of hiring an organic chemist, and subsequently for his generous advice and support throughout the last four years. I feel incredibly fortunate to have had the opportunity of his mentorship. I would like to thank all members of the Scanlon Materials Theory Group. In particular, special thanks go to Chris Savory for being the font of all learning, Dr Ben Williamson for his discerning taste in figures, Dr Katie Inzani for her knowledge of pre-1990s music, and Dr Adam Jackson for many in-depth discussions. Additional thanks go to the long-lost members of KLB room 349, including Dougal, Ian, Mia, and Rachel, for the constant supply of beer and conversation.

Prof. Aron Walsh and many of his current/ex-group members, including Jarvist, John, Keith, Dan, Chris, Suzy, and Lucy have been excellent fun at conferences and never stopped answering my Slack questions. Particular thanks go to Prof. Jamie Neilson and Annalise Maughan at CSU for making my visit so enjoyable. Prof. Brent Melot, Prof. Louis Piper, Dr Anna Regoutz, and Dr Ben Morgan deserve thanks for the number of drinks they have bought me at conferences.

This work was funded by EPSRC and Diamond Light Source through the EPSRC Centre for Doctoral Training in Molecular Modelling and Materials Science (EP/L015862/1). The computational work in this thesis was performed on a number of supercomputing centres: ARCHER UK National Supercomputing Service, via membership of the UK's HEC Materials Chemistry Consortium, funded by EPSRC (EP/L000202); Iridis cluster, provided by the EPSRC funded Centre for Innovation (EP/K000144/1 and EP/K000136/1); and the UCL Legion and Grace HPC facilities.

On a personal note, Nikul and Jack mean everything to me. Many thanks to DK and Standi in their own ways. Lastly, I would like to thank my family. My brothers, Ryan and Liam, for always being so sarcastic and keeping me in my place. My dad for his constant support. And my mother for everything she has done for me. 


\section{CONTENTS}

Publications $\quad$ xi

Notation $\quad$ xvi

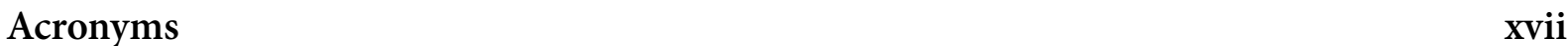

I Introduction 1

1 Photovoltaics 3

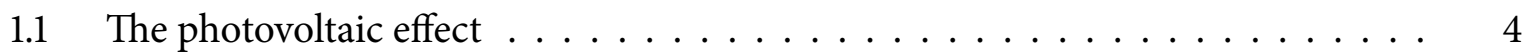

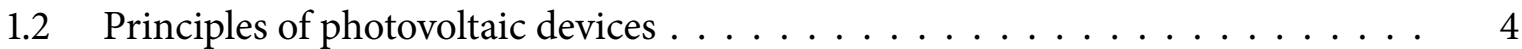

1.2.1 Key performance characteristics of solar cells . . . . . . . . . . . 6

1.2.2 Loss mechanisms in photovoltaic devices . . . . . . . . . 8

1.3 Desired solar absorber properties . . . . . . . . . . . . . . . . . . 9

1.3.1 Magnitude and nature of the band gap . . . . . . . . . . . . 10

1.3.2 Strength of optical absorption . . . . . . . . . . . . . . 10

1.3.3 Charge-carrier effective mass . . . . . . . . . . . . . 11

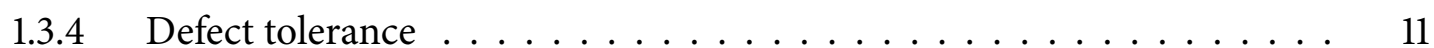

1.3.5 Dielectric constant and ferroelectric behaviour . . . . . . . . . . . . . 11

1.3.6 Rashba splitting . . . . . . . . . . . . . . . . . . . . . 12

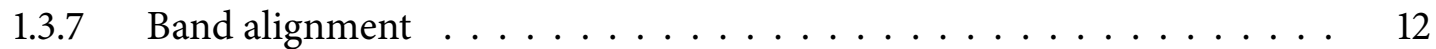

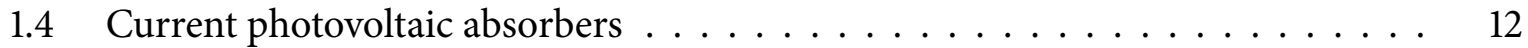

1.4.1 Third generation solar absorbers f . . . . . . . . . . . . . 13

1.4.2 Hybrid halide perovskites . . . . . . . . . . . . . . . . 14

1.4 .3 Emerging absorbers . . . . . . . . . . . . . . . . . 14

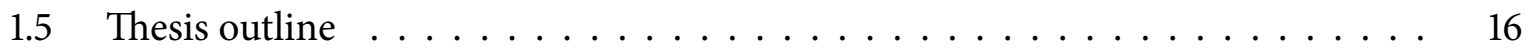

2 Computational theory $\quad 17$

2.1 Quantum mechanical approaches . . . . . . . . . . . . . . . 17

2.2 Hartree-Fock method . . . . . . . . . . . . . . . . . . . . . . . . 19

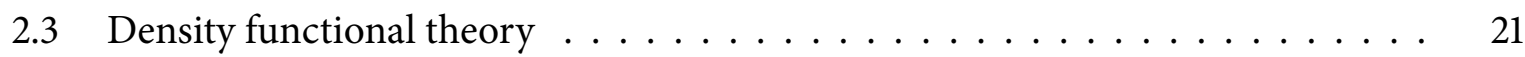

2.4 Density functional theory: implementation . . . . . . . . . . . . . . 23 
2.4.1 Exchange-correlation functionals . . . . . . . . . . 23

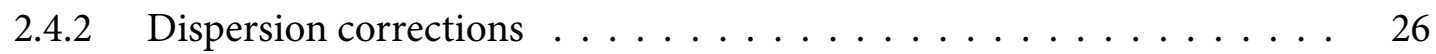

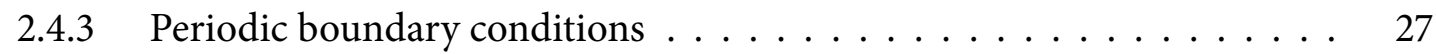

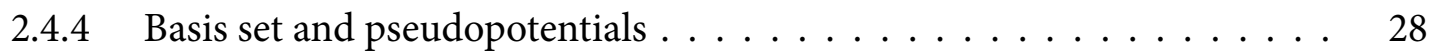

3 Computational methodology 31

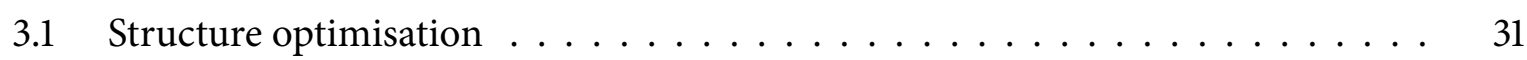

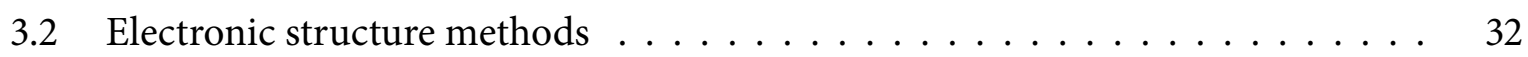

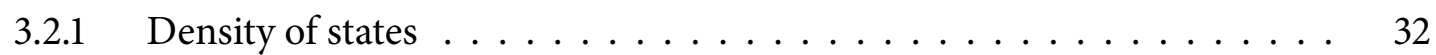

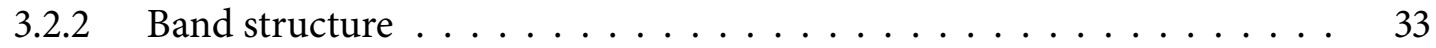

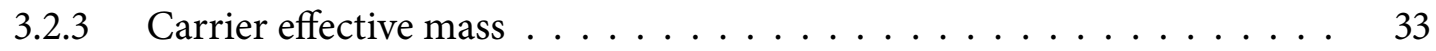

3.2 .4 Band alignment $\ldots \ldots \ldots \ldots \ldots \ldots \ldots \ldots \ldots$

3.2.5 Crystal orbital Hamilton population $\ldots \ldots \ldots \ldots . \ldots \ldots$

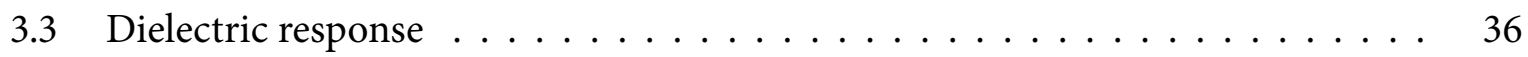

3.3.1 High-frequency dielectric spectra . . . . . . . . . . . 36

3.3.2 Ionic contribution to the dielectric constant . . . . . . . . . . . . 37

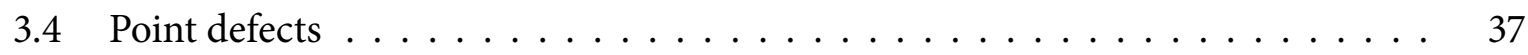

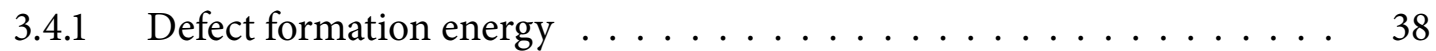

3.4.2 Thermodynamic transition levels . . . . . . . . . . . . . . . . 41

3.4.3 Chemical potential limits . . . . . . . . . . . . . . . 41

3.4.4 Transition level diagrams . . . . . . . . . . . . . . . . . 42

3.4 .5 Self-consistent Fermi level . . . . . . . . . . . . . . . . 43

3.4.6 Shockley-Read-Hall recombination rate . . . . . . . . . . . . . 44

3.5 Vienna $A b$ initio Simulation Package . . . . . . . . . . . . . . . . . 45

II Perovskite-inspired absorbers $\quad 47$

4 Review: perovskite photovoltaics 49

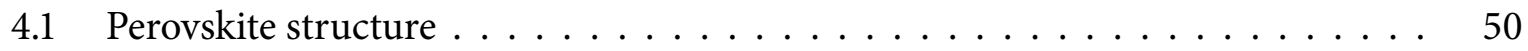

4.2 Reduced dimensionality perovskites . . . . . . . . . . . . . . . 52

4.3 Tin-based perovskites . . . . . . . . . . . . . . . . . . . . 54

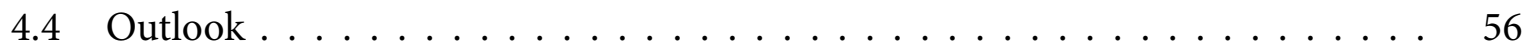

5 Pseudohalide perovskite absorbers $\quad 59$

5.1 Introduction . . . . . . . . . . . . . . . . . . . . . . . . 59 
5.2 Methodology ....................... 61

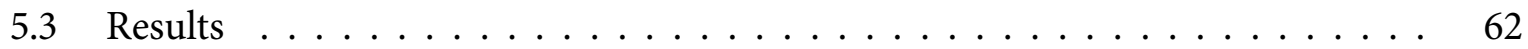

5.3.1 Geometric structure . . . . . . . . . . . . . . . . . . 62

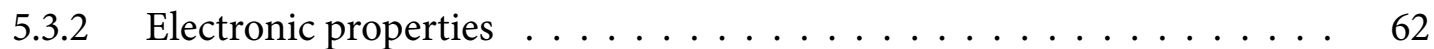

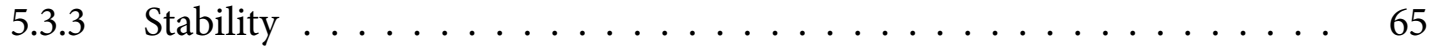

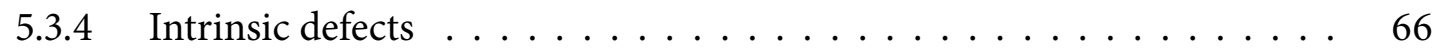

5.4 MAPSI structured analogues $\ldots \ldots \ldots \ldots \ldots \ldots \ldots \ldots$

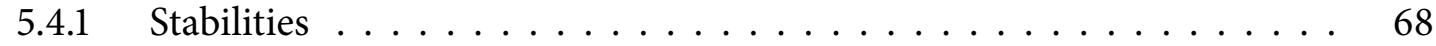

5.4 .2 Electronic properties . . . . . . . . . . . . . . . . . 69

5.4 .3 Dielectric constants . . . . . . . . . . . . . . . . . . . 74

5.4 .4 Band alignments . . . . . . . . . . . . . . . 75

5.4 .5 Defect chemistry . . . . . . . . . . . . . 76

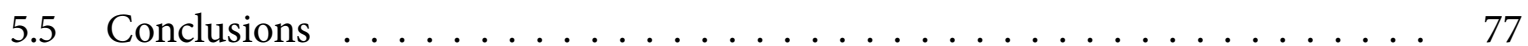

6 Vacancy-ordered double perovskites $\quad 79$

6.1 Introduction . . . . . . . . . . . . . . . . . . . . . 79

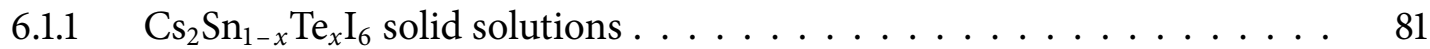

6.2 Methodology ......................... 83

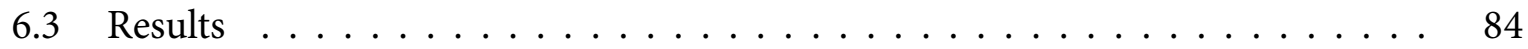

$6.3 .1 \quad$ Geometric structure . . . . . . . . . . . . . . . . . . . 84

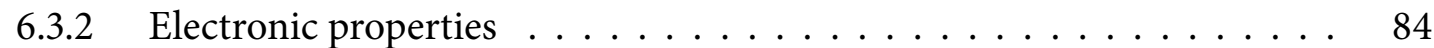

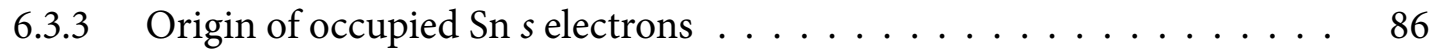

6.3.4 Nature of the $\mathrm{Cs}_{2} \mathrm{SnI}_{6}$ band gap . . . . . . . . . . . . . . . 89

6.3.5 Effective masses . . . . . . . . . . . . . . . . . 90

6.3.6 Reconciling small effective masses and ordered-vacancies . . . . . . . 91

6.3 .7 Band alignments . . . . . . . . . . . . . . . . 93

6.3.8 Defect chemistry . . . . . . . . . . . . . . . . . . 94

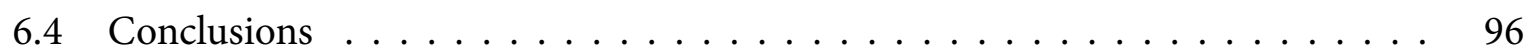

\section{Bismuth-based absorbers $\quad 99$}

7 Review: bismuth-based photovoltaics $\quad 101$

$7.1 \quad$ Bismuth sulfide . . . . . . . . . . . . . . . . . . . . . . . . . . . . . 101

7.2 Bismuth iodide . . . . . . . . . . . . . . . . . . 102 
7.3 Silver bismuth sulfide and iodide . . . . . . . . . . . . . . . . . 102

7.4 Caesium and hybrid bismuth iodides $\ldots \ldots \ldots \ldots \ldots \ldots$

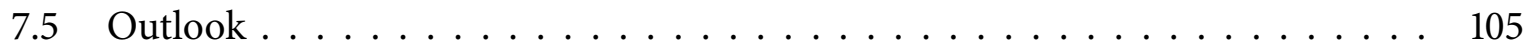

8 Bismuth chalcoiodides $\quad 107$

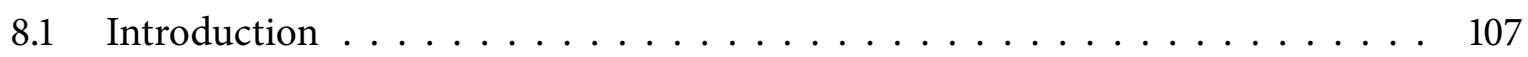

8.2 Methodology . . . . . . . . . . . . . . . . . . 108

8.3 Results . . . . . . . . . . . . . . . . . . . . . 109

8.3.1 Geometric structure . . . . . . . . . . . . . . . . . . . 109

8.3.2 Electronic properties . . . . . . . . . . . . . . . . . 110

8.3.3 The bismuth lone pair . . . . . . . . . . . . . . . . . . . 112

8.3.4 Effective masses and dielectric constants . . . . . . . . . . . . . . 114

8.3.5 Alignments with electron and hole contact layers . . . . . . . . . . . . 115

8.3.6 Defect chemistry . . . . . . . . . . . . . . . . . . 117

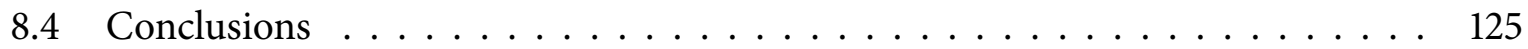

$\begin{array}{lll}9 & \text { Summary } & 127\end{array}$

$\begin{array}{ll}\text { Appendices } & 134\end{array}$

$\begin{array}{lll}\text { A Pseudohalide perovskite absorbers } & 134\end{array}$

B Vacancy-ordered double perovskites $\quad 139$

$\begin{array}{ll}\text { C Bismuth chalcoiodides } & 140\end{array}$

Indexes and Bibliography $\quad 144$

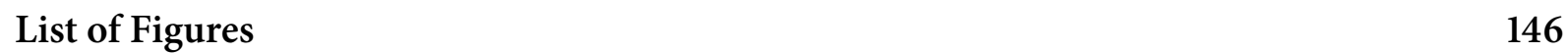

$\begin{array}{ll}\text { Bibliography } & 147\end{array}$ 


\section{PUBLICATIONS}

\section{Relating to thesis work}

1. Z. Wang, A. M. Ganose, C. Niu, and D. O. Scanlon, Two-dimensional hybrid perovskites for tunable energy level alignments and photovoltaics, submitted, (2018)

2. K. J. Fallon, P. Budden, E. Salvadori, A. M. Ganose, C. N. Savory, L. Eyre, D. O. Scanlon, C. W. M. Kay, A. Rao, R. H. Friend, A. J. Musser, and H. Bronstein, Exploiting excited-state aromaticity to design highly stable singlet fission materials, submitted, (2018)

3. A. M. Ganose, S. Matsumoto, J. Buckeridge, and D. O. Scanlon, Defect Engineering of Earth-Abundant Solar Absorbers BiSI and BiSeI, Chemistry of Materials, 30, 3827-3835 (2018)

4. A. E. Maughan, A. M. Ganose, M. A. Almaker, D. O. Scanlon, and J. R. Neilson, Tolerance Factor and Cooperative Tilting Effects in Vacancy-Ordered Double Perovskite Halides, Chemistry of Materials, 30, 3909-3919 (2018)

5. Z. Wang, A. M. Ganose, C. Niu, and D. O. Scanlon, First-principles insights into tin-based two-dimensional hybrid halide perovskites for photovoltaics, Journal of Materials Chemistry A, 6, 5652-5660 (2018)

6. A. E. Maughan, A. M. Ganose, A. M. Candia, J. T. Granger, D. O. Scanlon, and J. R. Neilson, Anharmonicity and Octahedral Tilting in Hybrid Vacancy-Ordered Double Perovskites, Chemistry of Materials, 30,472-483 (2018)

7. C. N. Savory, A. M. Ganose, and D. O. Scanlon, Exploring the $\mathrm{PbS}-\mathrm{Bi}_{2} \mathrm{~S}_{3}$ series for next generation energy conversion materials, Chemistry of Materials, 29, 5156-5167 (2017)

8. A. M. Ganose, C. N. Savory, and D. O. Scanlon, $\left(\mathrm{CH}_{3} \mathrm{NH}_{3}\right)_{2} \mathrm{PbI}_{2}(\mathrm{SCN})_{2}$ analogues for photovoltaic applications, Journal of Materials Chemistry A, 5, 7845-7853 (2017)

9. A. M. Ganose, C. N. Savory, and D. O. Scanlon, Beyond methylammonium lead iodide: prospects for the emergent field of $n s^{2}$ containing solar absorbers, Chemical Communications, 53, 20-44 (2017)

10. C. N. Savory, A. M. Ganose, W. Travis, R. S. Atri, R. G. Palgrave, and D. O. Scanlon, An Assessment of Silver Copper Sulphides for Photovoltaic Applications: Theoretical and Ex- 
perimental Insights, Journal of Materials Chemistry A, 4, 12648-12657 (2016)

11. A. E. Maughan, A. M. Ganose, M. M. Bordelon, E. M. Miller, D. O. Scanlon, and J. R. Neilson, Defect Tolerance to Intolerance in the Vacancy Ordered Double Perovskite Semiconductors $\mathrm{Cs}_{2} \mathrm{SnI}_{6}$ and $\mathrm{Cs}_{2} \mathrm{TeI}_{6}$, Journal of the American Chemical Society, 138, 8453-8464 (2016)

12. A. M. Ganose, M. Cuff, K. T. Butler, A. Walsh, and D. O. Scanlon, Interplay of Orbital and Relativistic Effects in Bismuth Oxyhalides: $\mathrm{BiOF}, \mathrm{BiOCl}, \mathrm{BiOBr}$ and $\mathrm{BiOI}$, Chemistry of Materials, 28, 1980 (2016)

13. W. Travis, C. Knapp, C. N. Savory, A. M. Ganose, P. Kafourou, X. Song, Z. Sharif, J. K. Cockcroft, D. O. Scanlon, H. Bronstein, and R. G. Palgrave, Hybrid Organic-Inorganic Coordination Complexes as Tunable Optical Response Materials, Inorganic Chemistry, 55, 3393 (2016)

14. A. M. Ganose, K. T. Butler, A. Walsh, and D. O. Scanlon, Relativistic Electronic Structure and Band Alignment of BiSI and BiSeI: Candidate Photovoltaic Materials, Journal of Materials Chemistry A, 4, 2060 (2016)

15. A. M. Ganose and D. O. Scanlon, Band gap and work function tailoring of $\mathrm{SnO}_{2}$ for improved transparent conducting ability in photovoltaics, Journal of Materials Chemistry C, 4, 1467 (2016)

16. A. M. Ganose, C. N. Savory, and D. O. Scanlon, $\left(\mathrm{CH}_{3} \mathrm{NH}_{3}\right)_{2} \mathrm{~Pb}(\mathrm{SCN})_{2} \mathrm{I}_{2}$ : a more stable structural motif for hybrid halide photovoltaics?, Journal of Physical Chemistry Letters, 6 , 4594 (2015)

\section{Not relating to thesis work}

17. Z. Wang, A. M. Ganose, C. Niu, and D. O. Scanlon, $\mathrm{Cd}_{2} \mathrm{Sb}_{2} \mathrm{O}_{7}$ : A high performance $n$-type thermoelectric with low lattice thermal conductivity, submitted, (2018)

18. A. J. Jackson, W. W. Leung, A. M. Ganose, I. J. Godfrey, M. Birkett, T. D. Veal, G. Sankar, R. G. Palgrave, and D. O. Scanlon, Hidden structural order in the new transparent conducting oxide $\mathrm{GaSbO}_{4}$, submitted, (2018)

19. A. Regoutz, A. M. Ganose, L. Blumenthal, C. Schlueter, T.-L. Lee, G. Kieslich, A. K. Cheetham, G. M. Vinai, T. Pincelli, G. Panaccione, H. K. Zhang, R. G. Egdell, J. Lischner, D. O. Scan- 
lon, and D. J. Payne, Insights into the Electronic Structure of $\mathrm{OsO}_{2}$, submitted, (2018)

20. N. H. Bashian, S. Zhou, A. M. Ganose, J. W. Stiles, A. Ee, D. S. Ashby, M. Zuba, D. O. Scanlon, L. F. Piper, B. Dunn, and B. C. Melot, Correlated Polyhedral Rotations in the Absence of Polarons During Electrochemical Insertion of Lithium in $\mathrm{ReO}_{3}, A C S$ Energy Letters, accepted (2018)

21. A. M. Ganose, A. J. Jackson, and D. O. Scanlon, sumo: Command-line tools for plotting and analysis of ab initio calculations, Journal of Open Source Software, 3, 717 (2018)

22. A. J. Jackson, A. M. Ganose, A. Regoutz, R. G. Egdell, and D. O. Scanlon, Galore: Broadening and weighting for simulation of photoelectron spectroscopy, Journal of Open Source Software, 3, 773 (2018)

23. A. M. Ganose, L. Gannon, F. Fabrizi, H. Nowell, S. Barnett, H. Lei, X. Zhu, C. Petrovic, D. O. Scanlon, and M. Hoesch, Local corrugation and persistent charge density wave in $\mathrm{ZrTe}_{3}$ with Ni intercalation Physical Review B, 97155103 (2018)

24. D. Biswas, A. M. Ganose, R. Yano, J. M. Riley, L. Bawden, O. J. Clark, J. Feng, L. CollinsMcintyre, M. T. Sajjad, W. Meevasana, T. K. Kim, M. Hoesch, J. E. Rault, T. Sasagawa, D. O. Scanlon, and P. D. C. King, Narrow-band anisotropic electronic structure of $\operatorname{ReS}_{2}$, Physical Review B, 96, 085205 (2017)

25. C. H. Hendon, K. T. Butler, A. M. Ganose, D. O. Scanlon, G. A. Ozin, and A. Walsh, Electroactive Nanoporous Metal Oxides and Chalcogenides, Journal of Materials Chemistry A, 29, 3663-3670 (2017)

26. N. F. Quackenbush, H. Paik, M. J. Wahila, S. Sallis, M. E. Holtz, X. Huang, A. M. Ganose, B. J. Morgan, D. O. Scanlon, Y. Gu, F. Xue, L.-Q. Chen, G. E. Sterbinsky, C. Schlueter, T.L. Lee, J. C. Woicik, J.-H. Guo, J. D. Brock, D. A. Muller, D. A. Arena, D. G. Schlom, and L. F. J. Piper, Stability of the M2 phase of vanadium dioxide induced by coherent epitaxial strain, Physical Review B, 94, 085105 (2016)

27. C. I. Hiley, D. O. Scanlon, A. A. Sokol, S. M. Woodley, A. M. Ganose, S. Angio, J. M. De Teresa, P. Manuel, D. D. Khalyavin, M. Walker, M. R. Lees, and R. I. Walton, Antiferromagnetism at $\mathrm{T}>500 \mathrm{~K}$ in the layered hexagonal ruthenate $\mathrm{SrRu}_{2} \mathrm{O}_{6}$, Physical Review B, 92, $104413(2015)$

28. Y. Hu, N. Goodeal, Y. Chen, A. M. Ganose, R. G. Palgrave, H. Bronstein and M. O. Blunt, 
PUBLICATIONS

Probing the Chemical Structure of Monolayer Covalent-Organic Frameworks Grown via Schiff-Base Condensation Reactions, Chemical Communications, 52, 9941-9944 (2016) 

notation

$$
\begin{aligned}
a() & \text { function } \\
a[] & \text { functional } \\
\{a\} & \text { set of all } a \\
\hat{a} & \text { operator } \\
\mathbf{a} & \text { vector }
\end{aligned}
$$$$
\text { Re real part }
$$

\section{functions}

$\delta$ Dirac delta function

$\Theta \quad$ Heaviside step function

\section{constants}

$$
\begin{aligned}
Z & \text { atomic number } \\
\hbar & \text { reduced Planck constant } \\
e & \text { electron charge } \\
k_{\mathrm{B}} & \text { Boltzmann constant } \\
m_{e} & \text { electron rest mass }
\end{aligned}
$$

\section{coordinates}

r Cartesian coordinate

R lattice

G reciprocal lattice

$\mathbf{x}$ generalised coordinate

k wave vector

\section{energies}

$E_{\mathrm{F}} \quad$ Fermi level

$E_{\mathrm{H}} \quad$ Hartree energy

$E_{\mathrm{g}}$ band gap

$E_{\mathrm{xc}}$ exchange-correlation energy

$T$ kinetic energy operators

$$
\begin{aligned}
\hat{H} & \text { Hamiltonian operator } \\
\hat{h} & \text { single-particle Hamiltonian operator } \\
\hat{T} & \text { kinetic energy operator } \\
\nabla^{2} & \text { Laplace operator }
\end{aligned}
$$

\section{potentials}

$V_{\mathrm{H}} \quad$ Hartree potential

$V_{\text {ext }}$ external potential

$V_{\mathrm{xc}}$ exchange-correlation potential

\section{thermodynamics}

$$
\begin{aligned}
\mu & \text { chemical potential } \\
T & \text { temperature } \\
\Delta_{\mathrm{f}} H & \text { formation energy }
\end{aligned}
$$

\section{electronic properties}

$$
\begin{aligned}
q & \text { charge } \\
g & \text { density of states } \\
\varepsilon_{\mathrm{r}} & \text { dielectric constant } \\
\alpha & \text { madelung constant } \\
\mu & \text { mobility } \\
V_{\mathrm{oc}} & \text { open-circuit voltage } \\
J_{\mathrm{sc}} & \text { short-circuit current } \\
m^{*} & \text { effective mass }
\end{aligned}
$$

\section{wavefunctions}

$$
\begin{array}{ll}
\Psi & \text { many-body wavefunction } \\
\chi & \text { single-particle wavefunction } \\
\psi & \text { Kohn-Sham wavefunction } \\
\phi & \text { atomic-like orbital } \\
\rho & \text { electron density }
\end{array}
$$




\section{ACRONYMS}

theory

DFT density functional theory

DFPT density functional

perturbation theory

HF Hartree-Fock

KS Kohn-Sham

SCF self-consistent field

SOC spin-orbit coupling

density functionals

GGA generalised gradient approximation

HSE06 Heyd-Scuseria-Ernzerhof functional

LDA local density approximation

PBE Perdew-Burke-Ernzerhof functional

PBEsol Perdew-Burke-Ernzerhof functional revised for solid

PBE0 Perdew-Burke-Ernzerhof hybrid functional

methods

D3 Grimme's D3 correction

PAW projector augmented wave

XRD X-ray diffraction

VASP Vienna $A b$ initio Simulation Package electronic properties

$\begin{aligned} \text { CB } & \text { conduction band } \\ \text { CBM } & \text { conduction band minimum } \\ \text { DOS } & \text { density of states } \\ \text { EA } & \text { electron affinity } \\ \text { IP } & \text { ionisation potential } \\ \text { VB } & \text { valence band } \\ \text { VBM } & \text { valence band maximum }\end{aligned}$

photovoltaic properties

AM 1.5G air mass 1.5 global spectrum

EQE external quantum efficiency

FF fill factor

SRH Shockley-Read-Hall

materials

Ch chalcogenide

HTM hole transporting material

MA methylammonium

FA formammidinium

FTO fluorine-doped tin oxide

TCO transparent conducting oxide

ITO tin-doped indium oxide

MAPI $\mathrm{CH}_{3} \mathrm{NH}_{3} \mathrm{PbI}_{3}$

MAPSI $\left(\mathrm{CH}_{3} \mathrm{NH}_{3}\right)_{2} \mathrm{~Pb}_{2} \mathrm{I}_{2}(\mathrm{SCN})_{2}$

P3HT poly(3-hexylthiophene)

$\mathrm{X}$ halide 

Part I

\section{Introduction}





\section{Photovoltaics}

The use of fossil fuels as an energy source has enabled the rapid industrialisation seen across the world since the 18th century. Today, the burning of oil, coal, and natural gas is at a record high and shows little sign of abating. Indeed, global energy consumption is expected to rise to over $30 \mathrm{PW}$ by 2050 from just $19 \mathrm{PW}$ in $2012 .{ }^{1}$ This inexorable demand for energy is problematic for several reasons: despite the relatively low cost of fossil fuels, their use is not sustainable due to finite reserves. Furthermore, and perhaps most pressing, the combustion of fossil fuels is the leading human source of greenhouses gases, which are detrimental both to the environment - where they are a major factor contributing towards global warming ${ }^{2}-$ and to the health of the population - where one in eight of total global deaths are linked to high levels of air pollution. ${ }^{3}$ Lastly, the uneven distribution of oil reserves creates significant challenges for nations without access to their own energy pipelines. Here, the European Union particularly suffers, with recent estimates suggesting that by 2020 over $80 \%$ of its energy supply will originate from outside its borders. ${ }^{4}$

There is, therefore, a growing demand for a clean energy source capable of providing sustainable economic growth. ${ }^{5}$ Of the currently available technologies, photovoltaics are perhaps the most promising due to the enormous amount of energy the sun can provide. Over 1,500 exawatt-hours of energy reach the earth in the form of solar radiation each year, dwarfing the total know reserves of oil, gas, and coal, which amount to fewer than 9 exawatt-hours. ${ }^{6,7}$ While current photovoltaic technologies have reached efficiencies high enough to capture a significant proportion of this energy, the cost of manufacturing these devices is a major roadblock preventing their widespread 
I Alongside the direct photoexcitation of electrons, a photovoltage can also arise due to light generated heating of a material. The resulting increase in temperature can produce a temperature gradient, which may create a voltage through the Seebeck effect. This mechanism is exploited in thermoelectrics - an emerging class of renewable energy materials. adoption. As such, there is a need for cost-effective solar cell technologies if photovoltaics are to compete in utility scale power generation. $^{8}$

\section{The photovoltaic effect}

The capacity of certain materials to produce a voltage and electric current upon exposure to light has been known for almost 200 years. It was first documented in 1839 by Edmond Becquerel, who noted a light-generated electric current between two platinum electrodes placed in a solution of sodium chloride. Subsequent experiments by Adams and Day in 1877 confirmed this effect arose from the properties of light rather than heat, ${ }^{\mathrm{I}}$ prompting them to term this phenomenon the photoelectric effect - today, referred to as the photovoltaic effect.

The origins of the photovoltaic effect in a semiconductor can be summarised as follows: the absorption of light results in the photoexcitation of an electron from the occupied valence band to the unoccupied conduction band, producing an electron-hole pair. If the pair is able to separate in space (termed exciton dissociation), both the electron and hole may behave as free charge carriers and contribute to the conductivity of the material by providing a photocurrent. Furthermore, as the electron and hole possess opposing charges and are separated in energy by the band gap of the semiconductor, the resulting potential difference produces a photovoltage. The existence of both a current and a voltage therefore allows for the generation of useable electrical power.

\section{Principles of photovoltaic devices}

The three main challenges required to efficiently harness the energy produced by the photovoltaic effect are:

1. To maximise the absorption of light and generate a large photocurrent. 
2. To maximise the potential difference between electron and hole and generate a large photovoltage.

3. To separate the electron-hole pair with high efficiency.

As we shall see later on, challenges 1 and 2 are interdependent and therefore must balanced against each other. The challenge presented by 3 is largely tackled through the formation of a $p-n$ junction, as used in a traditional solid-state solar cell.

A $p-n$ junction is formed when a $p$-type material (doped with excess holes) and an $n$-type material (doped with excess electrons) are brought into contact (Fig. 1.1). At the interface, some of the excess electrons in the $n$-type layer will diffuse into the $p$-type layer. Similarly, some of the excess holes in the $p$-type layer will diffuse into the $n$-type material. In the n-type layer, the diffusion of electrons leaves behind positively charged dopant atoms which are fixed in place in the crystal structure. Conversely, the diffusion of holes out of the $p$-type layer leaves behind negatively charged dopant sites. The resulting build-up of opposing charges at either side of the interface results in an electric field, which extends part way into the $n$ - and $p$ type layers. The area in which this field acts is termed the depletion region, since any charge carriers generated in this region are quickly swept out by the electric field - holes toward to the $p$-type layer and electrons toward the $n$-type layer. As such, the region itself is largely depleted of free carriers. As exciton dissociation will be considerably more efficient if photoexcitation occurs within the depletion region, the doped semiconductors comprising the $p-n$ junction are generally strong visible light absorbers.

a)

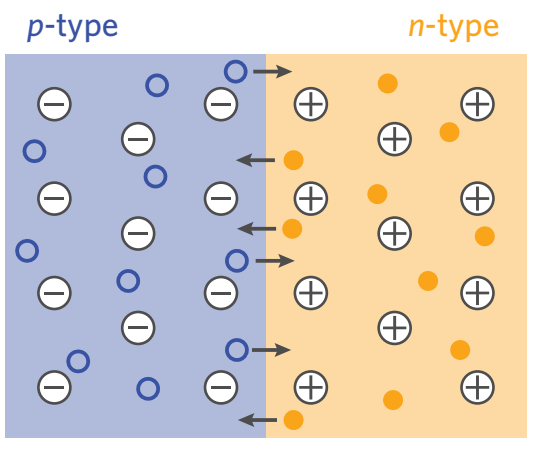

b)

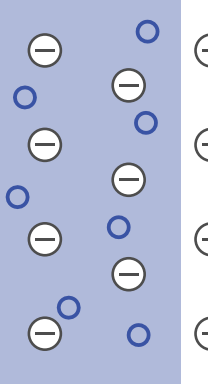

depletion region

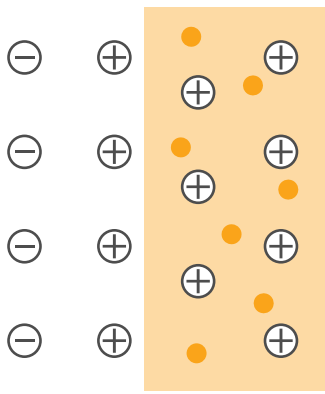

FIGURE 1.1: Simplified representation of a $p-n$ junction: a) Upon contact of $p$-type (shaded blue) and $n$-type (shaded orange) semiconductors, majority carriers (orange circles for electrons; empty blue circles for holes) diffuse across the interface. b) The diffusion leaves dopant ion cores exposed (negative ions in the $p$ type layer; positive ions in the $n$ type layer), leading to a build-up of opposing charges and a corresponding electric field at the interface. The region in which the electric field acts is termed the depletion region, since any free charge carriers will be quickly swept out. c) Photoexcitation results in the formation of an electron-hole pair. The field acts to separate the exciton, with the hole extracted to the $p$-type layer and electron extracted to the $n$ type layer.

c)

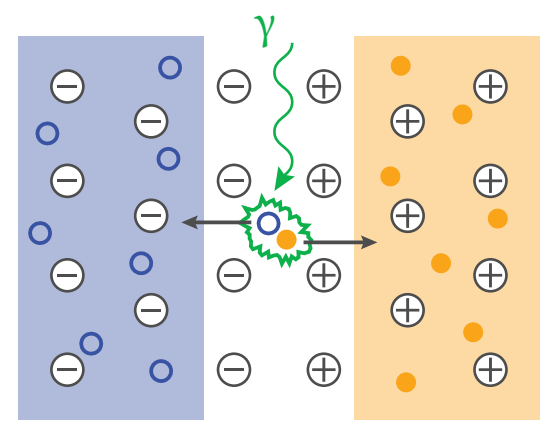




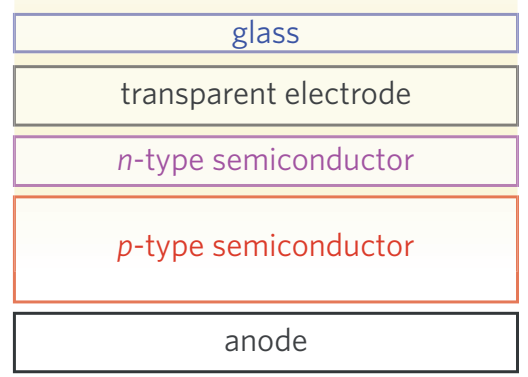

FIGURE 1.2: Schematic of a simplified $p-n$ junction solar cell.
To obtain useable electrical energy from a photovoltaic device, the separated charge carriers must be extracted out of the $p-n$ junction and into an external circuit. Solar cells, therefore, require highly conductive front and back contacts to enable the transport of electrons and holes out of the device. Traditionally, a metal is used as the back contact for collection of holes. The front contact poses an additional challenge in that it must be transparent to allow light into the device. As such, transparent conducting oxides, such as indium tin oxide (ITO) or $\mathrm{SnO}_{2}$, are employed as the $n$-type front contact layer. Many devices also contain buffer layers, which facilitate the transport of charge carriers to the electrodes. A simplified solar cell architecture is presented in Figure 1.2.

\section{Key performance characteristics of solar cells}

The performance of solar cells is quantified by the power conversion efficiency, $\eta$, which measures the percentage of power output, $P_{\max }$, to power input, $P_{\mathrm{in}}$, according to

$$
\eta=\frac{P_{\mathrm{max}}}{P_{\mathrm{in}}}=\frac{V_{\mathrm{oc}} J_{\mathrm{sc}} F F}{P_{\mathrm{in}}},
$$

II The AM $1.5 \mathrm{G}$ spectrum is a standard terrestrial reference spectra defined by the American Society for Testing and Materials. The spectra is based on the global average spectral irradiance if the Sun were at an angle of $42^{\circ}$ and the solar cell were at sea level, including losses due water, ozone, and other molecules in the atmosphere. where $V_{\mathrm{oc}}$ is the open-circuit voltage, $J_{\mathrm{sc}}$ is the short-circuit current, and FF is the fill factor. When measuring the efficiency of a photovoltaic, a set of standard conditions are employed to ensure consistency across different devices. In particular, measurements are performed at a temperature of $25^{\circ} \mathrm{C}$, using the AM $1.5 \mathrm{G}$ spectrum ${ }^{\mathrm{II}}$ as an illumination source, and with the incident power defined as $1 \mathrm{~kW} \mathrm{~cm}^{-2}$.

To optimise the efficiency of a solar cell it is essential to maximise the terms that contribute to the power output. The short-circuit current is the current passing through the cell under illumination when the contacts on either side of the cell are connected directly - resulting in zero voltage across the cell. The short-circuit current is strongly dependent on the ability of the absorbing layer to generate charge carriers. In an ideal device, every incident photon would be absorbed to produce an electron and hole that can contribute to the 
overall current. As only photons with energies greater than the band gap of the absorber can be absorbed, the largest short-circuit current will be achieved when then band gap is smallest.

The open-circuit voltage is the maximum voltage across the cell under illumination if the net current is zero - i.e. when the external circuit is not complete. The open-circuit voltage is greatest when there is the largest potential difference between the separated electrons and holes. As such, the open-circuit voltage is controlled primarily by the band gap of the semiconductor, with large band gaps allowing for greater potential difference between the charge carriers. The theoretical maximum open-circuit voltage of a device is roughly equivalent to the band gap of the absorber - assuming ohmic contacts with all buffer and transport layers. In practice, however, the open-circuit voltage is reduced by radiative and non-radiative carrier recombination, which act to increase the forward bias diffusion current and limit device performance.

The final performance characteristic required to calculate the power conversion efficiency is the fill factor, which is determined from the current-voltage $(J-V)$ curve of a cell under illumination. An example $J-V$ curve for a hypothetical cell is provided in Figure 1.3. The fill factor is a measure of the "squareness" of the $J-V$ curve and is defined as the ratio between the maximum power output versus the product of $V_{\mathrm{oc}}$ and $J_{\mathrm{sc}}$, according to

$$
F F=\frac{P_{\mathrm{max}}}{V_{\mathrm{oc}} J_{\mathrm{sc}}}=\frac{V_{\mathrm{mp}} J_{\mathrm{mp}}}{V_{\mathrm{oc}} J_{\mathrm{sc}}},
$$

where $V_{\mathrm{mp}}$ and $J_{\mathrm{mp}}$ are the voltage and current at the point of maximum power output. A fill factor closer to 1 indicates a highly ideal cell. In practice, recombination, interfacial effects, and other parasitic resistances generally result in a $J-V$ curve that deviates significantly from the perfect behaviour.

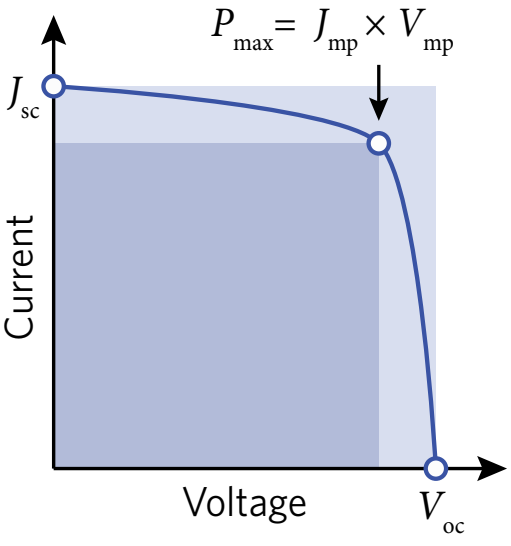

FIGURE 1.3: Example $J-V$ curve for a solar cell under illumination indicated by dark blue line, with the open-circuit voltage $\left(V_{\text {oc }}\right)$ and short-circuit current $\left(J_{\text {sc }}\right)$ highlighted. The dark blue square is defined by the location of the maximum power output $\left(P_{\max }\right)$, with the corresponding voltage and current at these points termed $V_{\mathrm{mp}}$ and $J_{\mathrm{mp}}$. The fill factor is the ratio between the dark and light blue squares. 


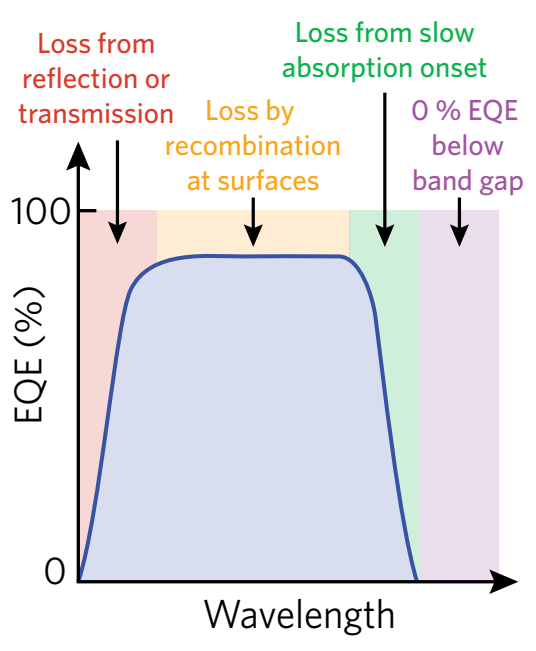

FIGURE 1.4: Example plot of external quantum efficiency (EQE) against photon wavelength, indicated by a solid blue line. Regions of the spectrum are highlighted to demonstrate potential optical loss mechanisms.

\section{Loss mechanisms in photovoltaic devices}

The performance of a solar cell is strongly impacted by device losses that can limit overall efficiency. When designing new absorber materials, it is crucial that these loss mechanisms are understood so their effects can be minimised. In solar absorbers, the dominating loss mechanisms are recombination of charge carriers, optical losses, and parasitic resistances that occur across the cell. Parasitic resistances are generally introduced during device fabrication and have minimal dependence on the intrinsic properties of the absorber or contact layers. As such, this thesis is mainly concerned with optical and recombination losses.

Optical losses manifest as reduction in short-circuit current and arise due to incomplete conversion of incident photons into separated electron-hole pairs. The degree of optical loss in a device is quantified by the external quantum efficiency (EQE) - namely, the ratio of the number of carriers collected by the solar cell versus the total number of photons incident on the device. The main sources of optical losses are transmission or reflection of visible light (Fig. 1.4). In particular, photons with energy below the band gap of the absorber will not be absorbed, resulting in an EQE of $0 \%$ for this energy range. Additional losses can result from front and rear surface reflection, and shading by the device electrodes.

Charge-carrier recombination is the process by which a metastable conduction band electron de-excites by combining with a hole in the valence band, thereby returning to equilibrium conditions. Recombination is detrimental for solar cell performance - by recombining, the free charge carriers cannot contribute the photocurrent (fewer charge carriers) and limit the photovoltage (increased dark forward bias current). The three most common types of electronhole recombination are presented in Figure 1.5 and are defined as:

Radiative Recombination Also termed spontaneous emission, here an excited electron combines with a hole, releasing a photon in the process. Radiative recombination can be considered the reverse of absorption and is the most common mode of recom- 
bination in direct band gap semiconductors.

Shockley-Read-Hall (SRH) Recombination All materials contain some concentration of defects (such as point defects, complexes, and grain boundaries), which can lead to the presence of isolated electronic states in the band gap. SRH or trap-assisted recombination is a two step process in which an electron (or hole) is trapped by the defect state, before a hole (or electron) is also trapped and the carriers recombine. If the trap is close in energy to the band edges (termed a shallow defect), thermal energy may be sufficient to promote the electron or hole back into the band where it can again act as a free carrier. As such, traps further away from the band edges, termed deep traps, are considerably more active as recombination centres.

Auger Recombination Here, an electron and hole recombine, however, rather than emitting a photon or phonon, the energy is given to an electron in the conduction band, promoting it to a further excited state. This electron will then thermalise to back to the conduction band edge. As this is a three-particle process, the probability of Auger recombination is significantly lower than for the two-particle recombination pathways, except in heavily doped systems.

In practice, the rates of radiative and Auger recombination are largely independent of materials synthesis and device engineering methods. In contrast, Shockley-Read-Hall recombination depends on the type and number of defects in the system, so may be controlled by doping, surface passivation, and synthesis technique. As such, attempting to tune the rate of Shockley-Read-Hall recombination is a primary strategy when optimising a potential photovoltaic material.

\section{Desired solar absorber properties}

It is difficult to predict how a novel solar absorber will perform in practice, due to the dependence of efficiency on many external conditions, including the deposition method, quality of precursors, and device architecture. However, studies into the best performing solar
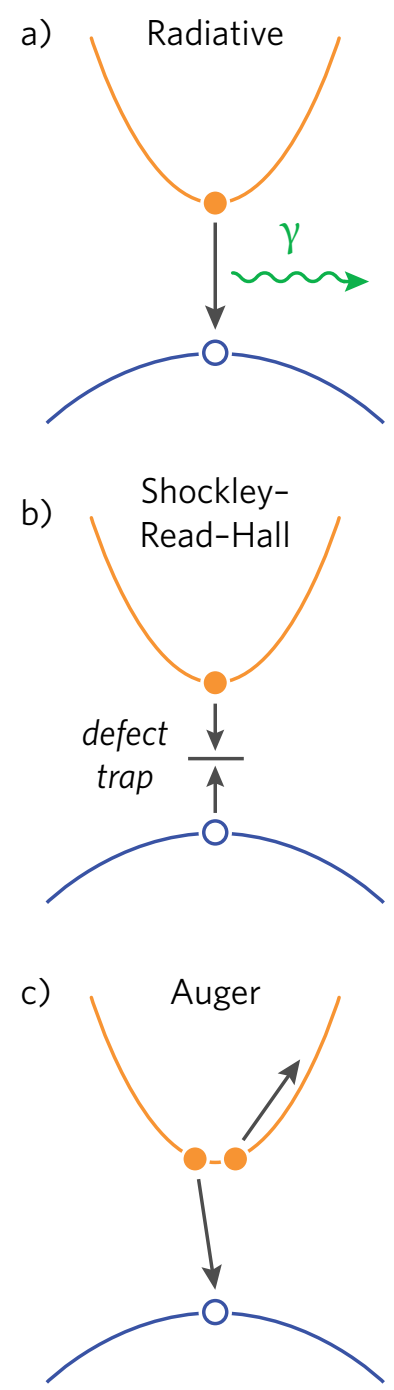

FIGURE 1.5: Representation of common electron-hole recombination mechanisms in photovoltaic devices: a) Radiative recombination, b) ShockleyRead-Hall recombination, and c) Auger Recombination. Valence and conduction bands indicated by blue and orange lines, respectively. Holes and electrons indicated by empty blue circles and filled orange circles, respectively. 


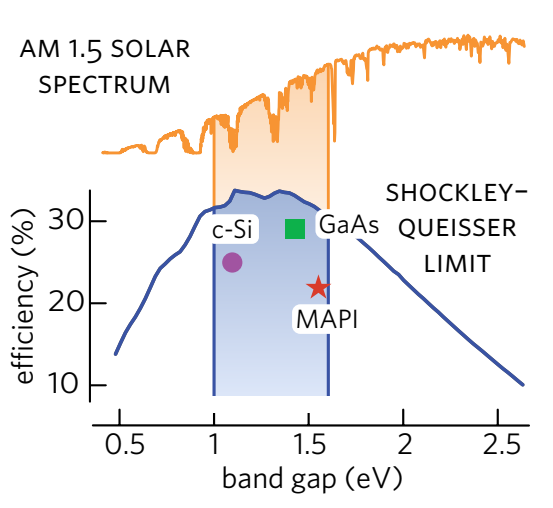

FIGURE 1.6: The optimal range of photovoltaic absorber band gaps (shaded) projected on to the AM 1.5 solar spectrum (yellow) and the Shockley-Queisser limit (blue).

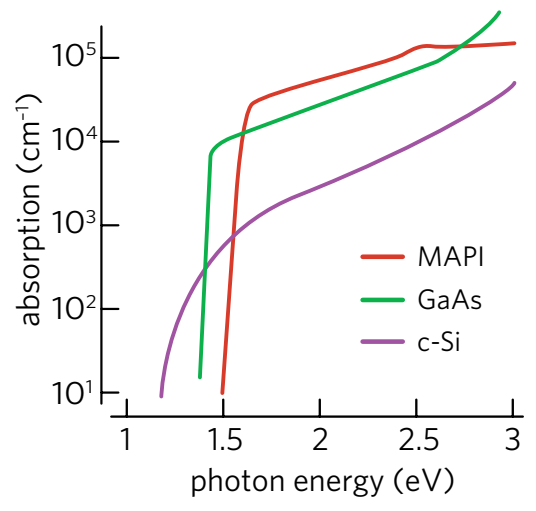

FIGURE 1.7: The importance of a direct fundamental band gap - crystalline silicon (c-Si, purple) has drastically reduced absorption compared to $\mathrm{CH}_{3} \mathrm{NH}_{3} \mathrm{Pbl}_{3}$ ( $\mathrm{MAPI}$, red) and $\mathrm{GaAs}$ (green). materials have revealed a number of key properties likely to result in efficient devices. ${ }^{9}$ Crucially, many of these properties, while difficult to measure experimentally, can be obtained relatively cheaply from theoretical methods, highlighting the importance of a combined experimental/theoretical approach when screening novel materials.

\section{Magnitude and nature of the band gap}

Perhaps the most important fundamental property of a solar absorber is its band gap. The Shockley-Queisser limit, ${ }^{10}$ which takes into account the antagonistic dependence of the short-circuit current $\left(J_{s c}\right)$ and open-circuit voltage $\left(V_{\text {oc }}\right)$ on the band gap and solar spectrum (Fig. 1.6), provides a theoretical cap on the maximum obtainable efficiency for a material. The highest performing absorbers possess direct band gaps in the range $1.0-1.6 \mathrm{eV}$ and while other materials may still be of interest, a band gap as close to $1.3 \mathrm{eV}$ is desired in order to maximise efficiency at around $33 \%$.

\section{Strength of optical absorption}

Strong optical absorption — characterised by a steep absorption edge in the absorption coefficient, $\alpha$, greater than $10^{4}-10^{5} \mathrm{~cm}^{-1}-$ is crucial if a solar absorber is to achieve high efficiencies (Fig. 1.7). ${ }^{11} \mathrm{In}$ deed, many compounds with ideal band gaps perform poorly due to weak absorption, typically as a result of an indirect band gap, such as in crystalline silicon. However, as recently highlighted by Yu and Zunger, indirect band gap materials may still perform well if a direct transition with an optimal energy is also available. ${ }^{12}$ Further reduction in absorption can occur if the fundamental band gap is dipole disallowed, thereby widening the optical band gap relative to the fundamental gap, as often seen in centrosymmetric systems. ${ }^{13,14}$ 


\section{Charge-carrier effective mass}

Large charge-carrier mobilities enable efficient electron-hole separation and are essential for high-performance devices. The mobility of electrons and holes, $\mu$, is related to the dispersion of the band edges in a material, with greater dispersion - or curvature - resulting in smaller effective masses and, in turn, greater charge-carrier mobilities (Fig. 1.8). Carrier mobilities are primarily limited through phonon and defect scattering processes. Minority-carrier lifetimes, $\tau$, have also recently been proposed as a further transport screening metric for novel PV materials, due to their role in Shockley-ReadHall recombination. ${ }^{9,15,16}$

\section{Defect tolerance}

Defect tolerance enables photovoltaics to retain strong optoelectronic properties, such as efficient carrier transport and power conversion efficiencies, despite the existence of defects. ${ }^{17}$ The bonding structure shown in Figure 1.9, whereby the valence band maximum is composed of antibonding interactions and the conduction band minimum is formed of bonding interactions, is thought to promote high levels of defect tolerance. In particular, it has been proposed that any dangling bonds formed due to vacancy defects will produce shallow states close to the band edges, rather than deep states in the middle of the band gap. ${ }^{18}$

\section{Dielectric constant and ferroelectric behaviour}

The electric response of a material is also vital in determining its photovoltaic performance. A large static dielectric constant indicates a high level of charge screening, enabling smaller defect chargecapture cross sections and reduced levels of non-radiative electronhole recombination. Additionally, in the case of "hydrogenic" defects, a large dielectric constant enables smaller defect binding energies, resulting in shallower defect levels. ${ }^{19}$ Ferroelectric photovoltaics

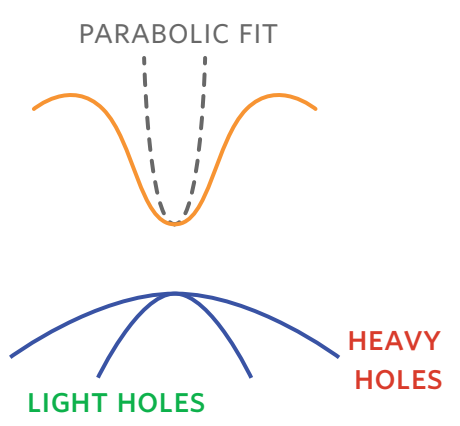

FIGURE 1.8: Band structure schematic demonstrating how increased band dispersion (curvature) results in smaller effective masses.
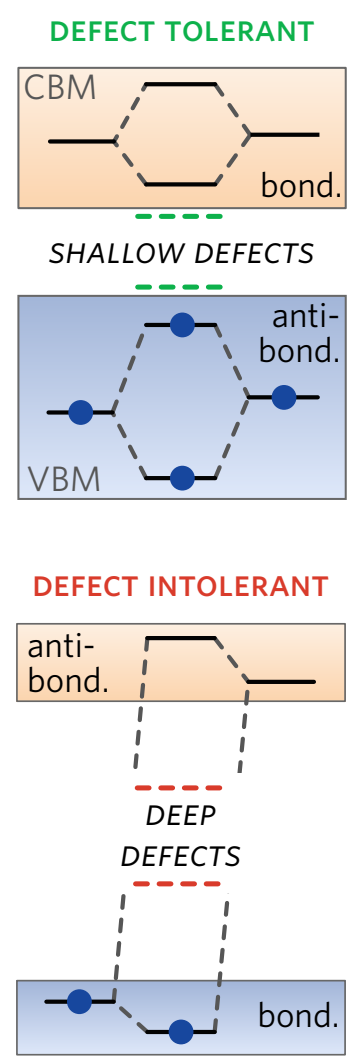

FIGURE 1.9: The importance of bonding structure on defect tolerance: an electronic structure with antibonding states at the valence band maximum is thought to promote shallow over deep defects. Adapted from ref. 18. 


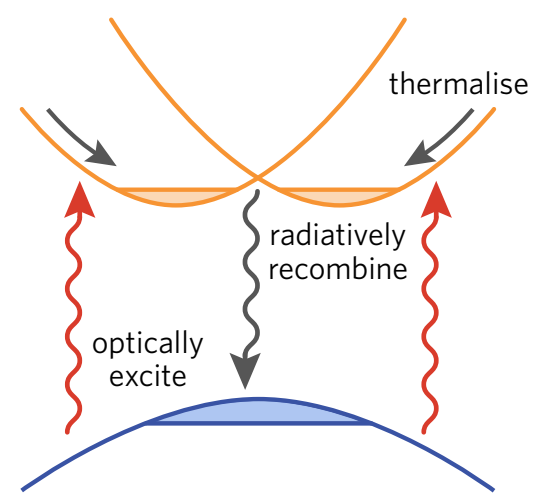

FIGURE 1.10: The processes of absorption and recombination in Rashba and Dresselhaus spinsplit systems. Adapted from ref. 26.

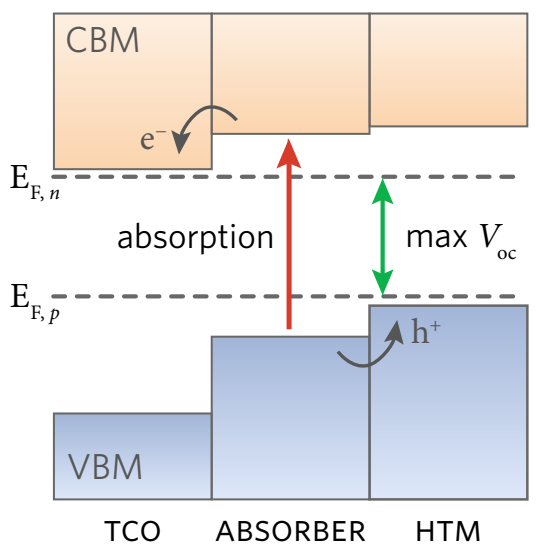

FIGURE 1.11: Schematic of band alignment in a heterojunction solar cell. Close alignment between components allows for maximisation of the open circuit voltage $\left(V_{\mathrm{oc}}\right)$. The Fermi level of the $n$ type transparent conducting oxide (TCO) and $p$-type hole transporting material (HTM) layers is denoted by $\mathrm{E}_{\mathrm{F}, n}$ and $\mathrm{E}_{\mathrm{F}, p}$, respectively. have undergone a revival in recent months due to the potential link between high levels of hysteresis and ferroelectricity in emerging hybrid halide perovskite absorbers. ${ }^{20-24}$

\section{Rashba splitting}

Hybrid-halide perovskite absorbers have recently made their mark on the emergent field of 'spintronics', due to the large Rashba and Dresselhaus splitting seen in their electronic band structures. ${ }^{25}$ Such a "spin-split indirect band gap" has been shown, both theoretically and experimentally, to dramatically reduce the rate of radiative recombination by a factor of more than $350 \%{ }^{26,27}$ Here, recombination is suppressed by a mismatch in electron momentum between the conduction band minima and valence band maximum, and is thought to enable the long charge-carrier lifetimes seen in the hybrid perovskites (Fig. 1.10). ${ }^{25,28}$

\section{Band alignment}

When manufacturing solar devices, judicious choice of electron and hole contact materials is essential to ensure favourable band alignment with the photovoltaic absorber. By obtaining an efficient band alignment, the open circuit voltage can be maximised and optimal carrier transport promoted (Fig. 1.11). In order to reduce costs and streamline the manufacturing process, ideal solar absorbers should align well with ubiquitous contact materials, such as indium tin oxide and $\mathrm{TiO}_{2}$.

\section{Current photovoltaic absorbers}

Most commercially available solar cells contain crystalline silicon as the absorber layer. Crystalline silicon devices have benefited from over four decades of research and possess efficiencies up to $26 \%$, ${ }^{29}$ however, their performance is limited by the indirect band gap of 


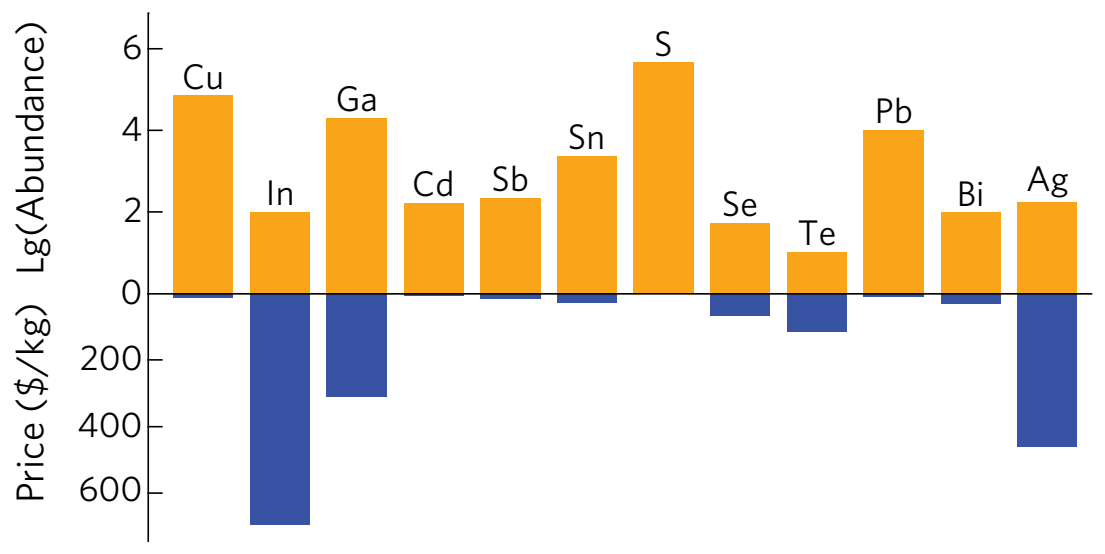

silicon, resulting in low absorption coefficients. ${ }^{30}$ Despite advanced manufacturing techniques such as localised back contacts and textured surfaces designed to maximise light capture, the efficiency of silicon devices has seen little improvement over the last five years. In addition, while the cost-per-panel of silicon cells has fallen steadily in recent years, this is mostly due to optimised fabrication methods and improved economies of scale. ${ }^{31,32}$ Further reduction in the price per Watt of panels is constrained by the underlying cost of producing silicon wafers, which is unlikely to change after over half a century of development in the transistor industry. These challenges have lead to the realisation of thin-film photovoltaics, in which use of a strongly absorbing material allows for thinner devices thereby reducing fabrication costs. These have shown record efficiencies up to $28.8 \%$ - for GaAs devices - but, due to the extremely high price of raw materials (Fig. 1.12), are limited to extraterrestrial applications where efficiency per gram takes precedence over cost. ${ }^{33,34}$

\section{Third generation solar absorbers}

The drawbacks of commercially available photovoltaics have prompted research into possible alternatives, collectively termed third generation materials. In order to be competitive with crystalline silicon, these materials require comparable efficiencies combined with dramatically reduced manufacturing costs. This has been approached primarily through use of low-cost and earth-abundant materials coupled with solution-processing synthesis methods. Third-generation
FIGURE 1.12: Comparison of elemental cost and abundance in the earth's crust. Data from the London Metal Exchange. 
devices include those based on $\mathrm{Cu}_{2} \mathrm{ZnSnS}_{4}$ (CZTS) and dye-sensitised solar cells employing a liquid electrolyte. Unfortunately, despite over ten years of development both technologies suffer from poor efficiencies that limit their commercial potential.

\section{Hybrid halide perovskites}

In 2009, lead hybrid halide perovskites emerged as a novel class of solar absorber, sparking a global race to ever-more-efficient devices. ${ }^{35,36}$ Cells containing the champion hybrid halide perovskite, $\mathrm{CH}_{3} \mathrm{NH}_{3} \mathrm{PbI}_{3}$ (MAPI), have been the subject of huge research interest, reaching efficiencies up to $22.7 \%$ in $2018 .{ }^{37}$ These record efficiencies have been enabled by a unique combination of properties, including a direct band gap of $1.55 \mathrm{eV},{ }^{38}$ small exciton binding energies, ${ }^{39}$ high levels of defect self-regulation, ${ }^{40}$ remarkably long charge-carrier diffusion lengths, ${ }^{41,42}$ and excellent charge-carrier mobilities. ${ }^{43,44}$ MAPI based devices are also cheap to produce due to low-temperature solutionprocessable synthesis routes and favourable band alignment with many commonly used hole and electron contact materials. ${ }^{45-47}$

Unfortunately, the commercialisation of hybrid halide solar cells is currently restricted by several key issues: critically, the sensitivity of the perovskite structure toward oxidation by water has led to the need for strict device encapsulation, necessarily increasing manufacturing costs. ${ }^{36}$ Furthermore, a growing number of reports suggest that the MAPI structure is intrinsically unstable, leading to decomposition even in the absence of moisture. ${ }^{48}$ As such, there are concerns over the leaching of lead into the environment posing a risk to human and aquatic life. While many studies have attempted to increase the stability of MAPI, this has so far proved challenging and remains a significant issue facing the hybrid perovskite community. ${ }^{49,50}$

\section{Emerging absorbers}

The success of the hybrid perovskites has energised the photovoltaic community. That a hitherto unknown material can surpass all other third-generation materials in such short a period of time highlights 


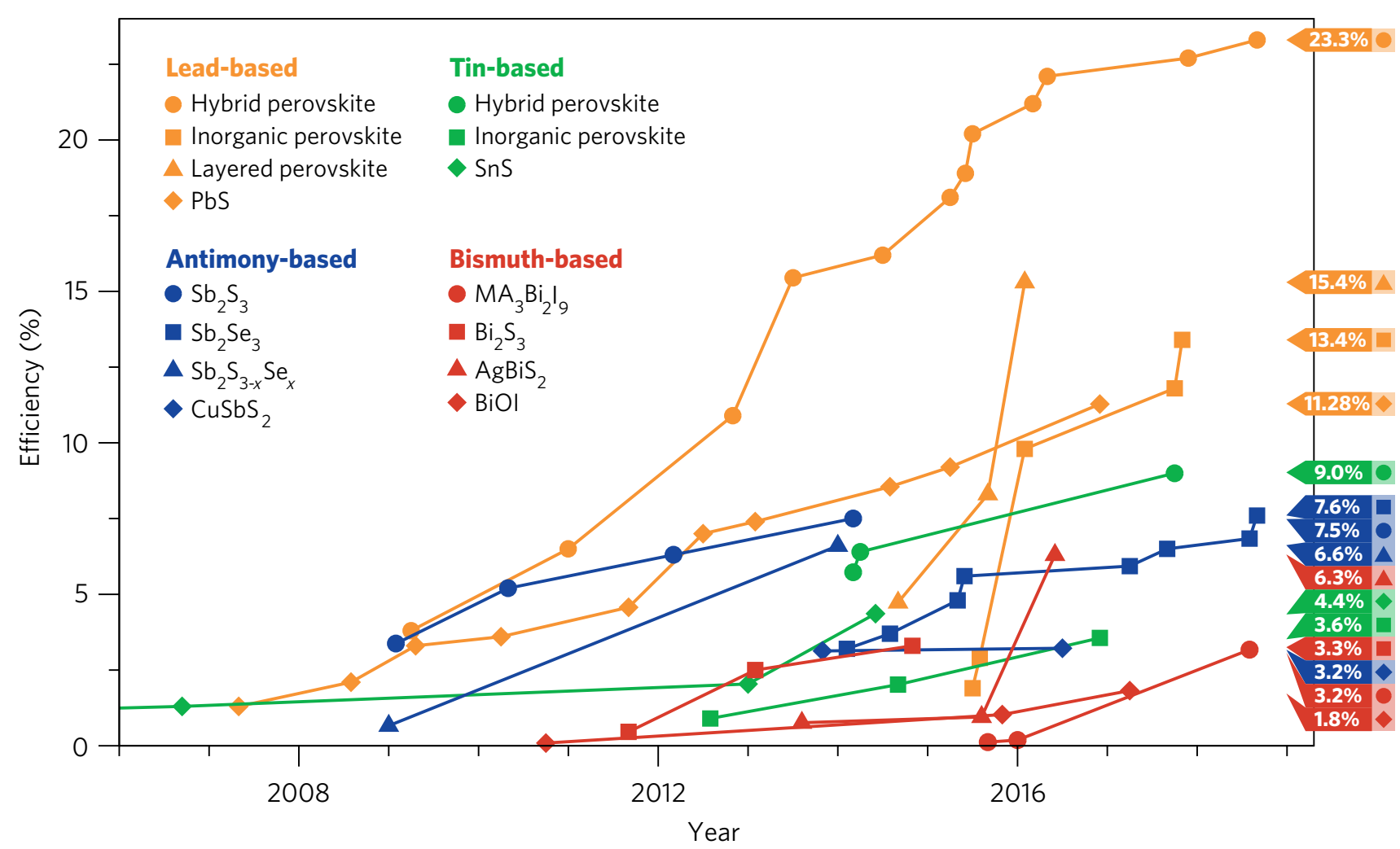

the potential for an earth-abundant and low-cost alternative to crystalline silicon. This rapid rise in performance has been based on an interdisciplinary approach combining a decades worth of developments in the synthesis, engineering, and photonics communities. Nevertheless, MAPI displays a fortuitous set of properties that enable remarkably efficient devices from films with high defect concentrations. As such, the speed of MAPI's success is likely to be an exception rather than a precedent to expect from all emerging absorbers. Indeed, any novel photovoltaic will likely require significant development before it can match the efficiency of the hybrid perovskites.

The last few years have seen the development of a broad array of emerging photovoltaic materials, several of which are highlighted in Figure 1.13. In particular, the layered hybrid perovskites, along with a variety of absorbers containing bismuth, antimony, and tin have shown promise, with significant efficiency improvements seen in the last 2-3 years. As such, there is currently tremendous scope for the discovery and optimisation of emerging solar absorbers.
FIGURE 1.13: Power conversion efficiency improvements over time for a selection of emerging photovoltaic absorbers. Lead-, tin-, antimony-, and bismuthbased absorbers are shown in orange, green, blue, and red, respectively. 


\section{Thesis outline}

This thesis investigates a number of materials for their suitability as solar absorbers for use in photovoltaics. Throughout, ab initio methods are used to provide insight into the structural, electronic, and optical properties that determine real-world performance. Special attention is paid to understanding the behaviour of intrinsic defects due to their critical role in determining recombination and charge transport.

The rest of Part 1 examines the theoretical basis and methodology of the calculations undertaken. The quantum theory underpinning the approach is discussed, including the limitations and advantages of the various methods used, before the implementation of these methods is outlined. In particular, the processes for obtaining accurate predictions of important photovoltaic properties, such as the band gap and optical absorption, are presented.

Part 2 is concerned with perovskite-related absorbers. The intrinsic instability of MAPI is addressed, before several strategies for improving the stability of perovskite-based devices are proposed. A novel layered perovskite is examined, based on which the existence of a new family of layered-perovskite materials - expected to show high efficiencies with improved thermodynamic stability - is predicted. In addition, the vacancy-ordered double perovskites are examined as potential lead-free perovskite photovoltaics.

In Part 3, the search for emerging photovoltaic absorbers is expanded beyond the hybrid perovskite family. The bismuth chalcohalides are investigated due to their earth-abundant and non-toxic nature. The electronic and defect properties of BiSI and BiSeI are calculated and used to map the rate of Shockley-Read-Hall recombination across chemical potential space. The implications of these results for experimental groups aiming to produce efficient bismuth chalcohalide photovoltaic devices is addressed. The final chapter of this thesis contains a summary of all findings and discusses the impact of this work on the field of photovoltaics. 


\section{COMPUTATIONAL THEORY}

\section{Quantum mechanical approaches}

All calculations presented in this thesis were performed using ab initio methods. These describe chemical systems by working up from fundamental physics, in contrast to empirical or semi-empirical approaches which rely on fitting to experimental measurements. The principle goal is finding a solution to the time-independent Schrödinger equation, ${ }^{51-53}$

$$
\hat{H} \Psi=E \Psi,
$$

where $\hat{H}$ is the Hamiltonian operator composed of kinetic and potential energy terms, $E$ is the system energy, and

$$
\Psi=\Psi\left(\mathbf{r}_{1}, \ldots, \mathbf{r}_{N}, \mathbf{r}_{1}^{n}, \ldots, \mathbf{r}_{M}^{n}\right)
$$

is a wavefunction that depends on the position of $N$ electrons $\{\mathbf{r}\}$ and $M$ nuclei $\left\{\mathbf{r}^{n}\right\}$. The Hamiltonian is of the form

$$
\begin{aligned}
\hat{H}= & -\overbrace{\frac{\hbar^{2}}{2 m_{e}} \sum_{i} \nabla_{i}^{2}}^{\hat{T}_{\text {elec }}}-\overbrace{\frac{\hbar^{2}}{2} \sum_{k} \frac{\nabla_{k}^{2}}{M_{k}}}^{\hat{T}_{\text {nuc }}}+\overbrace{\frac{1}{2} \sum_{i \neq j} \frac{e^{2}}{\left|\mathbf{r}_{i}-\mathbf{r}_{j}\right|}}^{V_{\text {elec-elec }}} \\
& +\underbrace{\frac{1}{2} \sum_{k \neq l} \frac{Z_{k} Z_{l}}{\left|\mathbf{r}_{k}^{n}-\mathbf{r}_{l}^{n}\right|}}_{V_{\text {nuc }- \text { nuc }}}-\underbrace{}_{\sum_{\text {nuc-elec }} \frac{e Z_{k}}{\left|\mathbf{r}_{i}-\mathbf{r}_{k}^{n}\right|}},
\end{aligned}
$$

where $\hbar$ is the reduced Planck constant, $i$ is the index over all electrons, $k$ is the index over all nuclei, $\nabla^{2}$ is the Laplacian operator, $e$ is the electron charge, $m_{e}$ is the electron rest mass, $M$ is the nuclear mass, and $Z$ is the nuclear charge. 
I Walter Kohn, a pioneer of quantum mechanical modelling, termed this challenge an "exponential wall" in his 1999 Nobel Prize lecture. ${ }^{54}$ Indeed, for a system containing 100 electrons, one would need to minimise a parameter space with $\sim 10^{150}$ dimensions.
Theoretically, finding an exact solution to the Schrödinger equation would allow one to calculate the exact ground state energy and wavefunction of a system. However, an analytic solution to the Schrödinger equation is only possible for systems containing a single atom with a single electron. While numerical methods allow for very accurate solutions up to several electrons, beyond this the number of parameters that must be optimised is so large as to be computationally intractable. ${ }^{I}$ As such, several approximations must be made, with the hope that the model retains physical relevance.

The first approximation is that the nuclei are considered stationary with respect to the movement of the electrons. This is termed the Born-Oppenheimer approximation and is generally valid given that the mass of the proton is $\sim 2000$ times the mass of the electron. This allows us to ignore the kinetic energy of the nuclei and rewrite the Hamiltonian as

$$
\hat{H}=\hat{T}_{\text {elec }}+V_{\text {nuc-elec }}+V_{\text {elec-elec }}+V_{\text {nuc-nuc }}
$$

where the potential energy of the nuclear interactions, $V_{\text {nuc-nuc }}$, is simply a constant for a static set of atomic coordinates. ${ }^{53}$ The terms describing the kinetic energy of the electrons, $\hat{T}_{\text {elec }}$, and the nuclearelectron interactions, $V_{\text {nuc-elec }}$, are relatively simple to evaluate. Calculating the potential energy between electrons, $V_{\text {elec-elec }}$, however, presents a significant challenge due to the many-body problem.

The variational principle is crucial to obtaining numerical solutions to the Schrödinger equation. It states that the expectation value of a Hamiltonian calculated using a trial wavefunction, $\Psi_{\mathrm{T}}$, will always be greater than the true ground state energy, $E_{0}$, produced from the true wavefunction, $\Psi_{0}$ :

$$
\left\langle\Psi_{\mathrm{T}}|\hat{H}| \Psi_{\mathrm{T}}\right\rangle=E_{\mathrm{T}} \geq E_{0}=\left\langle\Psi_{0}|\hat{H}| \Psi_{0}\right\rangle .
$$

Accordingly, by using an increasing number of trial wavefunctions we can obtain a more faithful description of the true wavefunction and convergence towards a more accurate total energy. 


\section{Hartree-Fock method}

To avoid the challenges posed by the many-body problem, in 1928 Hartree posited that the full multi-electron wavefunction could be approximated as a set of one-electron wavefunctions $(\chi)$ termed the Hartree product,

$$
\Psi\left(\mathbf{x}_{1}, \mathbf{x}_{2}, \ldots, \mathbf{x}_{N}\right)=\chi_{1}\left(\mathbf{x}_{1}\right) \chi_{2}\left(\mathbf{x}_{2}\right) \ldots \chi_{N}\left(\mathbf{x}_{N}\right),
$$

where $\mathbf{x}_{i}$ represents a generalised coordinate including the $x, y$, and $z$ cartesian coordinates and spin. He further introduced the idea of a one-electron Hamiltonian operator, $\hat{h}$, which acted on a one-electron wavefunction to produce the eigenequation

$$
\hat{h}_{i} \chi_{i}\left(\mathbf{x}_{i}\right)=\varepsilon_{i} \chi_{i}\left(\mathbf{x}_{i}\right)
$$

where $i$ is an electron occupying a single spin-orbital $\chi_{i}$, with energy $\varepsilon_{i}$, and the one-electron Hamiltonian takes the form

$$
\hat{h}_{i}=-\frac{\hbar^{2}}{2 m_{e}} \sum_{i} \nabla_{i}^{2}+\sum_{k} \frac{e Z_{k}}{\left|\mathbf{r}_{i}-\mathbf{r}_{k}^{n}\right|}
$$

The total energy of this non-interacting system is defined as the sum of the one-electron energies.

Due to the omission of electron-electron interactions, this model fails to realistically describe the behaviour of most systems. Crucially, the Hartree product does not obey the Pauli exclusion principle namely, that the wavefunction is antisymmetric with respect to the exchange of two fermions:

$$
\Psi\left(\ldots, \mathbf{x}_{1}, \mathbf{x}_{2}, \ldots\right)=-\Psi\left(\ldots, \mathbf{x}_{2}, \mathbf{x}_{1}, \ldots\right) .
$$

Subsequent work by Fock and Slater exploited the well-known signchanging property of matrix determinants. ${ }^{53,55}$ Rather than constructing the electronic wavefunction as a product of individual spin-orbitals, they noted that a single Slater determinant will instead produce a wavefunction that obeys the Pauli principle. For example, in a two- 
electron system:

$\Psi\left(\mathbf{x}_{1}, \mathbf{x}_{2}\right)=\frac{1}{\sqrt{2}}\left|\begin{array}{ll}\chi_{1}\left(\mathbf{x}_{1}\right) & \chi_{2}\left(\mathbf{x}_{1}\right) \\ \chi_{1}\left(\mathbf{x}_{2}\right) & \chi_{2}\left(\mathbf{x}_{2}\right)\end{array}\right|=\frac{1}{\sqrt{2}}\left[\chi_{1}\left(\mathbf{x}_{1}\right) \chi_{2}\left(\mathbf{x}_{2}\right)-\chi_{1}\left(\mathbf{x}_{2}\right) \chi_{2}\left(\mathbf{x}_{1}\right)\right]$

The antisymmetric nature of the wavefunction can be seen if the two electrons are exchanged:

$$
\begin{aligned}
\Psi\left(\mathbf{x}_{2}, \mathbf{x}_{1}\right) & =\frac{1}{\sqrt{2}}\left[\chi_{1}\left(\mathbf{x}_{2}\right) \chi_{2}\left(\mathbf{x}_{1}\right)-\chi_{1}\left(\mathbf{x}_{1}\right) \chi_{2}\left(\mathbf{x}_{2}\right)\right] \\
& =-\frac{1}{\sqrt{2}}\left[\chi_{1}\left(\mathbf{x}_{1}\right) \chi_{2}\left(\mathbf{x}_{2}\right)-\chi_{1}\left(\mathbf{x}_{2}\right) \chi_{2}\left(\mathbf{x}_{1}\right)\right] \\
& =-\Psi\left(\mathbf{x}_{1}, \mathbf{x}_{2}\right)
\end{aligned}
$$

Based on this, Hartree and Fock introduced two further terms to allow for calculation of the Hartree-Fock energy:

$$
\begin{aligned}
J_{i j} & =\frac{1}{2} \iint \chi_{i}\left(\mathbf{x}_{1}\right) \chi_{j}^{*}\left(\mathbf{x}_{2}\right) \frac{e^{2}}{\left|\mathbf{r}_{1}-\mathbf{r}_{2}\right|} \chi_{i}\left(\mathbf{x}_{1}\right) \chi_{j}^{*}\left(\mathbf{x}_{2}\right) \mathrm{d} \tau_{1} \mathrm{~d} \tau_{2} \\
K_{i j} & =\frac{1}{2} \iint \chi_{i}\left(\mathbf{x}_{1}\right) \chi_{j}^{*}\left(\mathbf{x}_{2}\right) \frac{e^{2}}{\left|\mathbf{r}_{1}-\mathbf{r}_{2}\right|} \chi_{j}\left(\mathbf{x}_{1}\right) \chi_{i}^{*}\left(\mathbf{x}_{2}\right) \mathrm{d} \tau_{1} \mathrm{~d} \tau_{2},
\end{aligned}
$$

where integrals with respect to $\mathrm{d} \tau$ represent integration over all space, $J_{i j}$ is the Coulomb term representing the electrostatic repulsion between electrons, and $K_{i j}$ is the exchange integral representing a quantum mechanical effect arising from the antisymmetric wavefunction. Electron exchange is a purely quantum mechanical effect driven by the Pauli principle. The Slater determinant wavefunctions ensure the exchange term will only be non-zero for electrons of the same spin, with the resulting exchange energy manifesting as a negative correction to the Coulomb energy.

The Hartree-Fock equations can then be written

$$
\left[\hat{h}_{i}+\sum_{i \neq j} J_{i j}-\sum_{i \neq j} K_{i j}\right] \chi_{i}=\varepsilon_{i} \chi_{i}
$$

The formulation of the Coulomb term simplifies the many-body problem of interacting electrons by adopting the mean field approximation. Here, each electron will move in an average potential field gen- 
erated by the presence of all other independent electrons. This allows one to solve the Hartree-Fock equations iteratively. Initially, a trial set of wavefunctions is chosen, from which the potential field for each electron is calculated. As the fields depend on the spin-orbitals of the other electrons, solving equation 2.13 will result in a new set of spinorbitals that can in turn be used to obtain a new set of potential fields. This procedure is repeated until the fields no longer change, resulting in a self-consistent field. To solve the entire system, the variational principle is employed to find the set of wavefunctions that produce the lowest energy.

While the Hartree-Fock method is still used today, its adoption of the mean-field approach results in a number of limitations. In particular, as each electron moves independently of the other electrons in the system, electron correlation - the property of electrons whereby they "avoid" each other - is ignored. ${ }^{56}$ As such, HartreeFock fails for systems containing localised electrons, such as transition metals. Furthermore, while the energy difference due to electron correlation generally amounts to only $\sim 0.1 \%$ of the total energy of a system, this is similar in magnitude to binding and reaction energies and can therefore lead to incorrect predictions.

\section{Density functional theory}

Density functional theory (DFT) takes an alternative approach in attempting to solve the total energy of a system. In 1964, Hohenberg and Kohn demonstrated that the ground state energy, along with all other ground state properties, is uniquely defined by the electron density, $\rho(\mathbf{r}) .{ }^{57}$ In contrast to Hartree-Fock theory, which relies on solving the wavefunction, density functional theory attempts to solve for the electron density. This presents a significant simplification as the density depends on just 3 variables ( $x, y$, and $z$ coordinates), whereas the wavefunction depends on $3 N$ variables, where $N$ is the total number of electrons in the system. The variational principle again applies, as the total energy calculated from the trial density will always be greater or equal to the true ground state energy. 
Hohenberg and Kohn proved that the total energy functional can be written

$$
E[\rho(\mathbf{r})]=\int \rho(\mathbf{r}) V_{\mathrm{ext}}(\mathbf{r}) \mathrm{d} \mathbf{r}+F_{\mathrm{HK}}[\rho(\mathbf{r})]
$$

where $V_{\text {ext }}$ is the external potential created by the nuclei and any external fields, and $F_{\mathrm{HK}}$ is the Hohenberg and Kohn universal functional containing all information about the kinetic energies and electronelectron interactions. Should the form of the universal functional be known, theoretically it should be possible to access the exact ground state properties of any system. In 1965, Kohn and Sham suggested a scheme for approximating $F_{\mathrm{HK}}$ by considering a fictitious non-interacting system of quasiparticles with an identical density to that of the fully interacting system of electrons. They started by separating the universal functional into interacting and non-interacting terms

$$
F_{\mathrm{HK}}[\rho(\mathbf{r})]=E_{\mathrm{H}}[\rho(\mathbf{r})]+T_{\mathrm{S}}[\rho(\mathbf{r})]+E_{\mathrm{xc}}[\rho(\mathbf{r})]
$$

where $E_{\mathrm{H}}$ is the classical (non-interacting) Hartree energy calculated as

$$
E_{\mathrm{H}}=\frac{e^{2}}{2} \iint \frac{\rho\left(\mathbf{r}_{i}\right) \rho\left(\mathbf{r}_{j}\right)}{\left|\mathbf{r}_{i}-\mathbf{r}_{j}\right|} \mathrm{d} \mathbf{r}_{i} \mathrm{~d} \mathbf{r}_{j},
$$

$T_{\mathrm{S}}$ is the kinetic energy of the non-interacting electrons, and $E_{\mathrm{xc}}$ is the sum of the non-classical electron-electron interactions and a correction to the kinetic energy accounting for non-classical interactions (thereby yielding the correct total kinetic energy).

To represent this system of non-interacting quasiparticles, Kohn and Sham reintroduced the idea of a wavefunction comprising a Slater determinant of Kohn-Sham wavefunctions, $\psi_{i}$. Crucially, this allowed for the exact solution of the non-interacting kinetic energy in the same manner as in Hartree-Fock

$$
T_{\mathrm{S}}[\rho(\mathbf{r})]=-\frac{\hbar^{2}}{2 m_{e}} \sum_{i}\left\langle\psi_{i}\left|\nabla^{2}\right| \psi_{i}\right\rangle .
$$

The density could therefore be written as the sum of the densities of the Kohn-Sham wavefunctions, which exactly recreate the density 
from the true multibody wavefunction

$$
\rho(\mathbf{r})=\sum_{i}\left|\psi_{i}(\mathbf{r})\right|^{2}=\left|\Psi_{\mathrm{mb}}(\mathbf{r})\right|^{2}
$$

By applying the appropriate variational condition, the one-electron Kohn-Sham equations can be written

$$
\left[-\frac{\hbar^{2}}{2 m_{e}} \nabla^{2}+e^{2} \int \frac{\rho\left(\mathbf{r}^{\prime}\right)}{\left|\mathbf{r}-\mathbf{r}^{\prime}\right|} \mathrm{d} \mathbf{r}-\sum_{k} \frac{e Z_{k}}{\left|\mathbf{r}-\mathbf{r}_{k}^{n}\right|}+V_{\mathrm{xc}}[\rho(\mathbf{r})]\right] \psi_{i}(\mathbf{r})=\varepsilon_{i} \psi_{i}(\mathbf{r}),
$$

where $V_{\mathrm{xc}}$ is the exchange-correlation functional related to the exchangecorrelation energy, $E_{\mathrm{xc}}{ }^{\text {II }}$ The ground state energy may then be solved in a self-consistent manner similar to as in the Hartree-Fock method. Initially, a set of trial wavefunctions are generated from which the density can be determined. This density is fed into equation 2.19 to produce a new set of Kohn-Sham wavefunctions and the process repeated until convergence is achieved.

While density functional theory is exact if the exact exchangecorrelation functional is known, the true functional form has not been elucidated and therefore must be approximated. As such, unlike Hartree-Fock which contains exact exchange and zero correlation, DFT instead obtains approximate exchange and approximate correlation. Despite this, DFT is widely employed in computational semiconductor research in both physics and chemistry communities.

\section{Density functional theory: implementation}

\section{Exchange-correlation functionals}

The design of the exchange-correlation functional is the primary factor in determining the accuracy of a DFT calculation. One of DFT's successes is that simple approximations to $E_{\mathrm{xc}}[\rho(\mathbf{r})]$ can produce accurate results. The most basic exchange-correlation functionals employ the local-density approximation (LDA), in which the amount of exchange and correlation at a given point is based solely on the local density - namely, the density of electrons at that point. The values

II It should be noted that the Kohn-Sham orbitals obtained from equation 2.19 need not resemble traditional chemical orbitals. Indeed, they can be any set of functions, provided they result in a density that minimises the total energy. 
of exchange and correlation have been extracted from high-precision quantum-Monte-Carlo simulations of uniform electron gases - hypothetic systems in which a number of electrons are free to move in an electrically neutral environment. ${ }^{58-60}$ In particular, the parameterisation of these calculations by Vosko, Wilk, and Nusair ${ }^{61}$ is often employed by DFT packages. The LDA is best suited for systems in which the electron density varies slowly but suffers from a number of failures when elements with tightly bound valence electrons, such as transition metals, are introduced. These manifest as an overbinding of electrons resulting in the overestimation of atomisation and ionisation energies and the underestimation of bond lengths.

Gradient-corrected functionals attempt to improve on the LDA by including not only the local density, $\rho(\mathbf{r})$, but also the gradient of the density, $\nabla \rho(\mathbf{r})$. A large variety of formulations exist; some are based solely on first-principles approaches whereas others are fitted to experimental data (semi-empirical functionals). In general, those which rely on local gradients within cutoff regions are termed generalised-gradient approximation (GGA) functionals. ${ }^{62}$

\section{$P B E$ and PBEsol}

Perdew, Burke, and Ernzerhof introduced their popular GGA functional, PBE, in 1996. ${ }^{63}$ PBE provides several improvements for periodic systems when compared to other GGA functionals but slightly underbinds electrons and therefore overestimates bond lengths. ${ }^{65}$ The PBEsol functional was released by Perdew and coworkers in 2008 as a version of the PBE functional revised for solids. ${ }^{64}$ By providing the functional with more "LDA-like" behaviour, PBEsol occupies a happy medium between the LDA and PBE, producing both better energies and lattice parameters in periodic systems. ${ }^{65} \mathrm{PBEsol}$ is employed extensively throughout this thesis.

Despite their widespread adoption, the LDA and GGA suffer from several issues which limit their accuracy. Primarily, both display the self-interaction error (SIE) in which an electron may spuriously interact with itself. This arises due to the use of the mean field approximation coupled with an approximate exchange-correlation functional. 
In contrast, Hartree-Fock avoids the SIE due to the exchange integral and Slater determinant basis (i.e. the presence of exact exchange) which prevent two electrons with the same spin from occupying the same position. This issue is most apparent for systems containing highly localised orbitals, such the first-row transition metals where there are a greater number of electrons in close proximity. The SIE leads to unphysical delocalisation of electrons in an attempt to minimise the degree of self-interaction. The use of approximate exchange and correlation also results in the underestimation of semiconductor band gaps - occasionally predicting known semiconductors to be metallic. ${ }^{66,67}$

\section{Hybrid DFT}

Hybrid functionals attempt to correct for the inaccuracies inherent in the LDA and GGA by introducing an amount of exact exchange from Hartree-Fock. Due to the expense of calculating the exchange term, this approach has only recently become computationally feasible in large periodic systems. Regardless, its use is quickly becoming the standard in the solid-state chemistry and physics communities. A commonly used non-parameterised hybrid functional is PBE0, ${ }^{68}$ formulated as

$$
E_{\mathrm{xc}}^{\mathrm{PBE} 0}=E_{\mathrm{xc}}^{\mathrm{PBE}}+\alpha\left(E_{\mathrm{x}}^{\mathrm{HF}}-E_{\mathrm{x}}^{\mathrm{PBE}}\right)
$$

where $\alpha$ is 0.25 based on perturbation theory. ${ }^{69}$

Due to the slow convergence of the exchange energy over distance, a number of range separated functionals have been developed in an attempt to reduce the computational cost associated with hybrid calculations. The Heyd-Scuseria-Ernzerhof (HSE06) functional is one such approach that has gained popularity. ${ }^{70,71}$ Here, only the short-range exchange energy is calculated using Hartree-Fock, with the remainder provided by $\mathrm{PBE}$, as

$$
E_{\mathrm{xc}}^{\mathrm{HSE}}=\alpha E_{\mathrm{x}}^{\mathrm{HF}, \mathrm{SR}}(\omega)+(1-\alpha) E_{\mathrm{x}}^{\mathrm{PBE}, \mathrm{SR}}(\omega)+E_{\mathrm{x}}^{\mathrm{PBE}, \mathrm{LR}}(\omega)+E_{\mathrm{c}}^{\mathrm{PBE}},
$$

where $\omega$ is $0.11 a_{0}{ }^{-1}$ and $\alpha$ is 0.25 . Accordingly, the functional tends 
to PBE0 as $\omega \rightarrow \infty$ and PBE as $\omega \rightarrow 0$. While ultimately a form of error cancellation, hybrid functionals have been shown to provide significant improvements over purely local based approaches. In particular, both PBE0 and HSE06 are able to accurately reproduce the low-temperature experimental band gaps of many semiconductors, often to within $0.1 \mathrm{eV} .{ }^{72}$ As HSE06 generally performs better for small and medium band gap materials such as photovoltaics, it has been employed for all high accuracy electronic structure calculations in this thesis.

\section{Dispersion corrections}

Most density functionals are unable to account for dispersive van der Waals type interactions as these arise from dynamical correlations between fluctuating charge distributions. These are particularly important for low-dimensionality materials, such as those containing planar sheets or nano-ribbons. In the density functional dispersion correction formalism (DFT-D), an additional term is added to the conventional Kohn-Sham total energy, ${ }^{73}$ as

$$
E_{\mathrm{DFT}-\mathrm{D}}=E_{\mathrm{KS}}+E_{\text {disp }}
$$

A large number of correction schemes have been proposed. ${ }^{74-78}$ In this thesis we have employed the DFT-D3 approach suggested by Grimme et al. ${ }^{76}$ in 2010 . Here, the dispersion correction is given by a pairwise sum over all atoms in the system (labelled by $i$ and $j$ ), as

$$
E_{\mathrm{disp}}=-\frac{1}{2} \sum_{i j} \sum_{n=6,8} \frac{C_{n}^{i j}}{\left|\mathbf{r}_{i}-\mathbf{r}_{j}\right|^{n}} f_{d, n}\left(\left|\mathbf{r}_{i}-\mathbf{r}_{j}\right|\right),
$$

where $C_{n}^{i j}$ are the averaged geometry-dependent $n$ th-order dispersion coefficients for the $i j$ atom pair, calculated based on fitting to time-dependent DFT calculations, and $f_{d, n}$ is a damping function to avoid singularities for small internuclear distances and doublecounting at intermediate distance $s$, where the damping is parameterised based on the choice of exchange-correlation functional. In particular, we have adopted the zero damping D3 correction. DFT- 
D3 has been shown to result in improved forces and geometries for a wide range of solid state materials. ${ }^{76}$ The Axilrod-Teller triple dipole terms were not included in this work.

\section{Periodic boundary conditions}

It is not practical to solve the Schrödinger equation for macroscopic materials due to the large number of particles they contain. We can instead take advantage of the crystalline nature of many solid-state materials by considering a much smaller repeating unit. Here the geometric arrangement of atoms in a macroscopic solid is approximated as an infinitely repeating perfect crystal. The smallest repeating set of atoms, termed the motif, is located on the lattice points of a three-dimensional Bravais lattice, which is periodically repeated in all three dimensions to form the overall structure (Fig. 2.1). The structure can be defined in terms of a unit cell, a parallelepiped cell that can be tiled to build the whole crystal. While there are an infinite number of possible unit cells for a given lattice, crystallographic conventions describe two classes of cell: the primitive cell is a unit cell containing exactly one lattice point, and the conventional cell is the smallest possible unit cell possessing the same symmetry as the overall lattice and may contain more than one lattice point. Both cells will produce the same crystal structure when repeated throughout space. To reduce computational cost the primitive cell is used for all calculations.

The cell is described by a set of lattice vectors, $\mathbf{a}, \mathbf{b}$, and $\mathbf{c}$, with the lattice containing all periodic images given by

$$
\mathbf{R}=n_{1} \mathbf{a}+n_{2} \mathbf{b}+n_{3} \mathbf{c}
$$

where $\left\{n_{1}, n_{2}, n_{3}\right\}$ are integers. The lattice vectors determine the cell shape, such as the cell angles $\alpha, \beta$, and $\gamma$. While translational symmetry is required in all Bravais lattices, the cell may possess additional symmetry in the arrangement of the atoms. Accordingly, the wavefunction will experience the same symmetry constraints, often enabling a further reduction in computational cost.

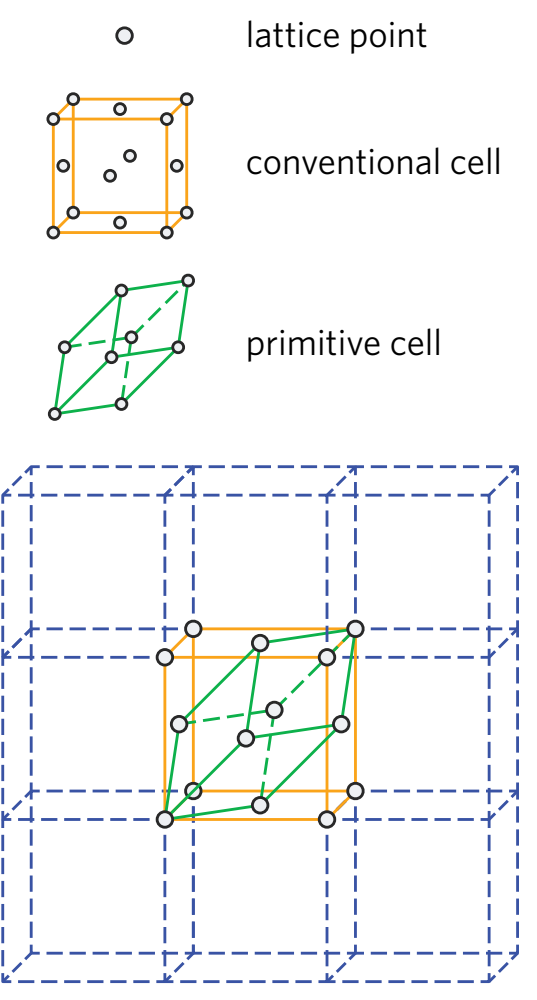

FIGURE 2.1: Representation of the primitive cell (green), conventional cell (orange), and lattice (blue) of the face centred cubic structure, indicating the effects of periodic boundary conditions. 
The use of Bloch's theorem enables us to simplify the solution of the one-electron wavefunctions under periodic boundary conditions. It states that the wavefunction of particle in a periodic potential is formed of

(a) a function, $u_{\mathbf{k}}(\mathbf{r})$, with the periodicity of the lattice, and

(b) a plane wave $\mathrm{e}^{\mathrm{ik \mathbf {r }} \text {. }}$

The wavefunction of electron $n$ with wave vector $\mathbf{k}$ may therefore be written

$$
\psi_{n, \mathbf{k}}(\mathbf{r})=u_{n, \mathbf{k}}(\mathbf{r}) \mathrm{e}^{i \mathbf{k} \cdot \mathbf{r}} .
$$

Due to the periodicity of the lattice, any wave vector $\mathbf{k}^{\prime}$ that differs from $\mathbf{k}$ by an integer number of reciprocal lattice vectors will result in an identical solution. Accordingly we can restrict the values of $\mathbf{k}$ to those occurring in smallest repeating unit of the reciprocal lattice, termed the first Brillouin zone.

When studying periodic systems through HF or DFT, one must therefore find the set of periodic functions that minimises the total energy in a normalised sum over all $\mathbf{k}$-vectors. As an infinite number of $\mathbf{k}$-vectors exist, an approximation must be made in the form of discrete k-point sampling. As the wavefunction varies smoothly within $\mathbf{k}$-space, this approximation will hold provided the space is sampled with sufficient density. In this thesis we have adopted the Monkhorst-Pack method, whereby an equally spaced grid of points are sampled. In all cases the grid is centred at the origin of reciprocal space, termed the $\Gamma$ point. Semiconductors and insulators generally require relatively low sampling densities, whereas metallic systems necessitate dense k-point meshes so that the Fermi level can be accurately determined. To ensure calculations remain accurate without excess computational cost, the $\mathbf{k}$-point mesh density is systematically increased until convergence criteria are met.

\section{Basis set and pseudopotentials}

The remaining challenge is to determine the form of the periodic potential, $u(\mathbf{r})$. In most $a b$ initio approaches, the potential is represented as a linear combination of basis functions. The choice of 
these functions is a primary factor in determining the accuracy and cost of a calculation. A plane wave basis set is a natural choice for periodic calculations, in part due to their equivalent representation as a Fourier series. ${ }^{79}$ As the plane wave basis is only dependent on the size of the simulation cell, it will not suffer from basis set superposition errors in contrast to localised basis sets such as Gaussian functions.

Since the potential has the same periodicity as the lattice, it can be expressed as a linear combination of plane waves with wavevectors $\mathbf{G}$ that are reciprocal lattice vectors of the crystal

$$
u_{n}(\mathbf{r})=\sum_{\mathbf{G}} c_{n, \mathbf{G}} \mathrm{e}^{i \mathbf{G} \cdot \mathbf{r}}
$$

where $c_{n, \mathbf{G}}$ are the plane wave coefficients. Together with equation 2.25 , the one-electron wavefunctions can be written

$$
\psi_{n, \mathbf{k}}(\mathbf{r})=\sum_{\mathbf{G}} c_{n, \mathbf{k}+\mathbf{G}} \mathrm{e}^{i(\mathbf{k}+\mathbf{G}) \cdot \mathbf{r}}
$$

While in principle use of a plane wave basis set is exact given an infinite number of plane waves, in practice a finite-sized basis set must be used. This is achieved by defining a plane wave energy cutoff, with the plane wave energy defined by

$$
E_{\mathbf{k}+\mathbf{G}}=\frac{\hbar^{2}}{2 m_{e}}|\mathbf{k}+\mathbf{G}|^{2}
$$

Large cutoff energies are needed to represent wavefunctions where the electron density oscillates rapidly - for example, the electrons near atomic cores. The cutoff leads to another advantage of using a plane wave basis set, in that the accuracy of the basis can controlled through a single parameter - a significant simplification compared to localised basis sets. Similar to the $\mathbf{k}$-space sampling density, the cutoff energy is increased until convergence is reached.

The wavefunctions of valence electrons oscillate rapidly close to the nucleus due to the strong nuclear potential and the requirement that they be orthogonal to the core electrons. Accordingly, a large number of high energy plane waves are required to accurately model these electrons, significantly increasing the computational cost. As 


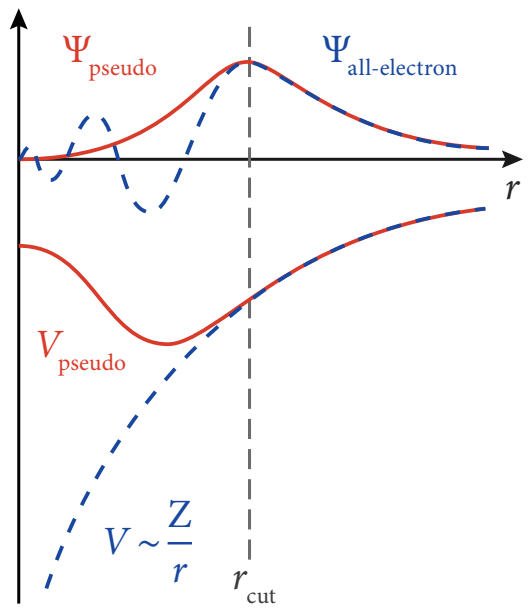

FIGURE 2.2: Schematic representation of the relationship between an all electron (blue dashed line) and pseudopotential (solid red line) wavefunction $(\Psi)$ and potential $(V)$. The psuedo-wavefunction replicates the all-electron wavefunction outside the cutoff, $r_{\text {cut }}$. the core electrons only interact weakly with the valence electrons, it is convenient to approximate their behaviour by use of an effective potential representing a screened nuclear core. This is achieved through use of pseudopotentials. These are smoothly varying functions that produce pseudo-wavefunctions with the same shape as the "true" wavefunction outside a certain cutoff but with fewer nodes within it (Fig. 2.2). The pseudopotentials are carefully fitted for each element based on all-electron calculations, and are constructed such that the scattering properties of the pseudo-wavefunctions are the same as the scattering produced by the ion and core electrons. As such, pseudopotentials are generally angular momentum dependent. Use of pseudopotentials makes calculations involving large atoms with many core electrons tractable but reduces the accuracy of results. ${ }^{80}$

The calculations performed in this thesis employ the projector augmented-wave method (PAW). Developed by Blöchl in $1994,{ }^{81}$ this method aims to enable the physics of all-electron calculations at a pseudopotential-like computational cost. ${ }^{82}$ Here, the core electrons are represented by localised basis sets, with the effects of the core states projected onto the valence electrons. As such, the correct nodal behaviour of the valence electron wavefunctions is maintained. Allelectron scalar relativistic calculations are used to generate the PAW fixed core states. 


\section{Computational}

\section{METHODOLOGY}

\section{Structure optimisation}

The potential produced by any known exchange-correlation functional will always deviate from the true potential. As such, the ground state bond lengths and geometry of a material will not match those determined from experiment, even if measured at $0 \mathrm{~K}$. It is therefore considered good practice to optimise a structure before further calculations are performed. For solid-state materials, both the positions of the nuclei and the lattice parameters must be converged. This is achieved by

1. calculating the forces acting on each atom,

2. perturbing the atoms to reduce the residual force, and

3. repeating the above until the forces fall within a certain tolerance.

The simplest technique for evaluating the atomic forces involves displacing each nuclei in all directions to find the force numerically. It is easy to see, however, that for large systems this approach will quickly become impractical. Instead, the Hellmann-Feynman theorem is employed, ${ }^{83}$ which states that if the distribution of the electrons is known, the atomic forces may be obtained from classical electrostatics. This enables the forces to be calculated from the wavefunction directly. The Hellmann-Feynman theorem can also be applied in calculating the stress tensor, thereby allowing for relaxation of the unit cell shape and volume. The quasi-Newton Raphson RMM-DIIS 
and conjugate gradient algorithms ${ }^{84}$ were used to perturb the nuclei to their ground state positions once the atomic forces had been obtained.

This approach to complete structural optimisation leads to the unwanted introduction of Pulay stress. ${ }^{85}$ When the volume of the lattice changes during relaxation, the plane wave basis set is no longer complete, resulting small errors in the diagonal components of the stress tensor. Pulay stress can be avoided by running a series of fixedvolume optimisations and fitting the energies to an equation of state to obtain the equilibrium volume. Alternatively, the plane wave energy cutoff can be raised significantly above that required to converge the total energy, at which point the Pulay stress becomes negligible. In this thesis we have employed the latter approach, with the converged cutoff energy increased by $\sim 30 \%$ for geometry optimisations.

\section{Electronic structure methods}

With the ground state geometry obtained, the electronic structure can then be characterised using a number of methods. Many of these are useful for assessing a compound's suitability as a photovoltaic absorber.

\section{Density of states}

The density of states (DOS) is a measure of the number of electronic states (per unit volume of a system) as a function of energy. The density of states can be compared with experimental spectra obtained from techniques such as X-ray photoelectron spectroscopy (XPS) and hard X-ray photoelectron spectroscopy (HAXPES). The total density of states can be decomposed into the contributions from individual atoms or orbitals, termed the projected density of states. Here, the wavefunction is projected onto the spherical harmonics for each atom within a certain radius - in our calculations defined by the PAW projection radii. In this thesis, all density of states spectra have been broadened to improve their legibility. This was achieved via convolution with a gaussian function with a width of $0.2 \mathrm{eV}$. In this 
thesis, density of states were plotted using the sumo package developed by Ganose and Jackson. ${ }^{86}$

\section{Band structure}

Band structure diagrams are a plot of the electronic eigenvalues as a function of wave vector $(\mathbf{k})$. The band structure provides crucial information about the electronic properties of a system. For example, in semiconductors and insulators, analysis of the frontier valence and conduction bands indicates the magnitude and nature (direct vs indirect) of the fundamental band gap. In order to reduce the computational cost, it is essential to choose a path through $\mathbf{k}$-space that will be representative of the reciprocal lattice and capture as many interesting phenomena as possible. A common technique is to plot a path along the high-symmetry lines of the first Brillouin zone. These paths are unique for each crystal class. We have used the coordinates of the high-symmetry points defined in Bradley and Cracknell. ${ }^{87}$ Band structures were constructed and plotted using the sumo package. ${ }^{86}$

\section{Carrier effective mass}

The band structure of a material also provides information about the effective mass of charge carriers. In particular, bands displaying a greater degree of dispersion at the band edges give rise to charge carriers with smaller effective mass, in comparison to flat bands which indicate localised states. The effective mass of a charge carrier, $m^{*}$, at the band edges is calculated as

$$
\frac{\hbar}{m^{*}}=\frac{\mathrm{d}^{2} E(\mathbf{k})}{\mathrm{d}^{2}}
$$

where $\mathrm{d}^{2} E(\mathbf{k}) / \mathrm{dk}^{2}$ is the curvature of the band at the band edge. Anisotropy in the band curvature was taken into account through parabolic fitting of the band in all high symmetry directions. The relationship between effective mass and carrier mobility, $\mu$, is given by

$$
\mu=\frac{e \tau}{m^{*}}
$$


where $e$ is the elementary charge and $\tau$ is the average scattering time. The calculation of $\tau$ is computationally demanding since it depends on the doping and defect properties of a system. Accordingly, in this thesis we provide the effective mass as an indicator of potential mobility. Effective masses were calculated using the sumo package. ${ }^{86}$

\section{Band alignment}

A limitation of periodic electronic structure calculations is the absence of any external reference energy. In order to align the electronic eigenvalues to the vacuum level - and thus calculate the ionisation potential and electron affinity - further calculations are required. In this thesis, we have adopted the core-level alignment approach of Wei and Zunger, ${ }^{217}$ whereby:

1. A slab model is constructed containing a significantly large vacuum region such that periodic images do not interact.

2. The electrostatic potential is averaged in the direction perpendicular to the surface of the slab. The potential in the middle of the vacuum region is taken as the vacuum level $\left(E_{\mathrm{vac}}\right)$.

3. The energy of a core state deep in the slab is taken as a reference $\left(E_{\text {core }}^{\text {slab }}\right)$. The slab should be thick enough that this reference state is a good approximation for "bulk-like" behaviour.

4. The reference energies are compared against the valence band maximum $\left(E_{\mathrm{vbm}}\right)$ and an equivalent core energy $\left(E_{\text {core }}^{\text {bulk }}\right)$ taken from a calculation on the bulk material.

The ionisation potential (IP) can then be calculated according to

$$
\mathrm{IP}=\left(E_{\mathrm{vac}}-E_{\text {core }}^{\text {slab }}\right)-\left(E_{\mathrm{vbm}}-E_{\text {core }}^{\text {bulk }}\right)
$$

The planar average of the calculated electrostatic potential was extracted and plotted using the MACRODENSITY package. ${ }^{88-91}$

\section{Crystal orbital Hamilton population}

Crystal orbital Hamilton population (COHP) analysis provides information on the strength and nature of bonding in a system. By 
rewriting the total energy as a sum of orbital pair contributions, an energy resolved COHP diagram can be produced - effectively a partitioned density of states. The sign of the COHP indicates the bonding type - with positive and negative values indicating bonding and anti-bonding, respectively - whereas the magnitude indicates the bond strength, with larger values indicating stronger interactions.

The COHP method was originally proposed by Blöchl to aid the analysis of bonding in periodic calculations employing a localised basis set. ${ }^{92}$ Recently, Deringer et al. ${ }^{93}$ showed that by projecting the wavefunction onto a set of localised orbitals, $\left\{\phi_{\mu}\right\}$, the COHP method could be extended for use in plane wave calculations. Here, the transfer matrix, $\mathbf{T}$, quantifies the overlap between the band wavefunctions, $\psi_{j}$, and the local orbitals, as

$$
T_{j \mu}(\mathbf{k})=\left\langle\psi_{j}(\mathbf{k}) \mid \phi_{\mu}\right\rangle
$$

As the COHP is given between pairs of orbitals, the aim is to calculate the bonding strength between an orbital, $\mu$, located at the first atom involved in the bond in question, and another orbital, $v$, on the second atom. To achieve this, the projected density matrix, $\mathbf{P}^{\text {proj, is }}$ defined as

$$
P_{j \mu \nu}^{\mathrm{proj}}(\mathbf{k})=T_{j \mu}^{\dagger}(\mathbf{k}) T_{j v}(\mathbf{k})
$$

The Hamiltonian must be expressed in the basis of the local functions. ${ }^{94}$ This is achieved by expanding the plane wave Hamiltonian, $\hat{H}^{\mathrm{pw}}$, according to

$$
\begin{aligned}
\hat{H}_{\mu \nu}^{\mathrm{proj}}(\mathbf{k}) & =\left\langle\phi_{\mu}\left|\hat{H}^{\mathrm{pw}}\right| \phi_{\nu}\right\rangle \\
& =\sum_{j}\left\langle\phi_{\mu} \mid \psi_{j}(\mathbf{k})\right\rangle \varepsilon_{j}(\mathbf{k})\left\langle\psi_{j}(\mathbf{k}) \mid \phi_{\nu}\right\rangle
\end{aligned}
$$

Using equation 3.4 we can write

$$
\hat{H}_{\mu \nu}^{\mathrm{proj}}(\mathbf{k})=\sum_{j} \varepsilon_{j}(\mathbf{k}) T_{j \mu}^{\dagger}(\mathbf{k}) T_{j v}(\mathbf{k})
$$

Finally, the energy-dependent projected COHP (pCOHP) can be 
calculated as

$$
\operatorname{pCOHP}_{\mu \nu}(E, \mathbf{k})=\sum_{j} \operatorname{Re}\left[P_{j \mu \nu}^{\mathrm{proj}}(\mathbf{k}) \hat{H}_{\mu \nu}^{\mathrm{proj}}(\mathbf{k})\right] \times \delta\left(\varepsilon_{j}(\mathbf{k})-E\right)
$$

where $\delta$ is the Dirac delta function. Accordingly, the projected density matrix has been transformed into a density-of-states matrix. The real-space $\mathrm{pCOHP}(E)$ is calculated by summing over all orbitals $(\mu$ and $v$ ) and integrating over $\mathbf{k}$-space.

In this report, pCOHP analysis was performed using the LOBSTER program, in which the methodology above has been adapted for use in the PAW formalism. ${ }^{95,96}$ The tetrahedron method was employed when integrating over $\mathbf{k}$-space. ${ }^{81,97}$ The remaining challenge is in choosing a suitable basis set of localised functions. LOBSTER is provided with a minimal basis set of Slater-type orbitals, fitted to atomic wavefunctions, that possess the correct nodal behaviour in the core region. ${ }^{98,99}$ The basis set further provides additional basis functions for unoccupied atomic orbitals based on fitting to plane wave DFT free-atom calculations. ${ }^{96}$

\section{Dielectric response}

The dielectric response quantifies a material's ability to screen charge. The dielectric response at low-frequencies is termed the static dielectric constant. A large static dielectric constant is thought to improve defect tolerance by screening the effects of charged defects on carrier transport and non-radiative recombination. The static dielectric constant is comprised of contributions from both the electron density and the lattice.

\section{High-frequency dielectric spectra}

The electronic component is obtained in the calculation of the highfrequency dielectric spectra. Obtaining the high-frequency dielectric response of a material is also crucial for understanding its optical properties. The process by which this is achieved within the PAW formalism is not trivial and has been addressed in detail in 
Gajdoš et al. ${ }^{100}$ Here, the imaginary part of the dielectric constant $\left(\varepsilon_{\mathrm{r}}^{\prime \prime}\right)$ is obtained by a summation over all direct valence band to conduction band transitions. Accordingly, this method fails to account for indirect and intraband transitions. The real part of the dielectric response $\left(\varepsilon_{\mathrm{r}}^{\prime}\right)$ is then obtained from the imaginary part by the Kramers-Kronig transformation. This single-particle approach does not include electron-hole correlations and other many-body effects. Accordingly, for very accurate descriptions of the dielectric response, higher order methods such as the Bethe-Salpeter equation should be employed. ${ }^{101}$ Regardless, this approach has previously been shown to provide reasonable agreement with experiment. ${ }^{102}$

\section{Ionic contribution to the dielectric constant}

The lattice contribution depends on the Born effective charges - effectively a measure of how much charge follows an atom when it is displaced - and the phonon modes at the $\Gamma$ point. These properties can be obtained either through finite displacements or density functional perturbation theory (DFPT), with the methodology behind both approaches covered in detail in Baroni and Resta ${ }^{103}$, Gonze and Lee ${ }^{104}$, and Nunes and Gonze ${ }^{105}$. In thesis we have employed the DFPT approach due to the reduced computational cost.

\section{Point defects}

The presence of defects is the primary factor controlling conductivity and recombination in solar absorbers. Defects are usually distributed randomly throughout a material and are often present at low concentrations. To model the effects of point defects in solid-state calculations, we have employed the supercell approximation. Here, a defect is introduced into an expansion of the unit cell containing multiple primitive cells (termed a supercell), with the aim of simulating a defect in solution. Crucially, it is essential to minimise the interaction between a defect and its images in neighbouring cells. In practice, the supercell size is limited based on the availability of computational resources. 


\section{Defect formation energy}

The formation energy, $\Delta_{\mathrm{f}} H$, of a defect, $X$, with charge state $q$, is calculated as

$$
\Delta_{\mathrm{f}} H^{X, q}=\left(E^{X, q}-E^{\mathrm{H}}\right)+\sum_{i}\left[n_{i}\left(E_{i}+\mu_{i}\right)\right]+q\left(E_{\mathrm{F}}+\varepsilon_{\mathrm{vbm}}^{\mathrm{H}}\right)+E_{\mathrm{corr}},
$$

where $E^{X, q}$ is the energy of the defected supercell and $E^{\mathrm{H}}$ is the energy of the unperturbed host supercell. The second term represents the energy change due to losing an atom, $i$, to a chemical reservoir: $n_{i}$ is the number of atoms of each type lost, $E_{i}$ is the element reference energy calculated from the element in its standard state, and $\mu_{i}$ is the chemical potential of the atom, which can be used to explore typical growth conditions. The first two terms can be understood simply as an expression of Hess' Law - i.e. the formation energy is effectively the energy of the products minus the reactants. The third term is only required when calculating the formation energy of a charged defect and accounts for the exchange of charge carriers with a carrier reservoir: $\varepsilon_{\mathrm{vbm}}^{\mathrm{H}}$ represents the energy needed to add or remove an electron from the valence band maximum to a Fermi reservoir - i.e. the eigenvalue of the valence band maximum in the host - and $E_{\mathrm{F}}$ is the Fermi level relative to $\varepsilon_{\mathrm{vbm}}^{\mathrm{H}}$. $E_{\text {corr }}$ is a correction applied to account for various limitations of the defect scheme used and is comprised of three terms,

$$
E_{\text {corr }}=E_{\mathrm{pot}}+E_{\mathrm{bf}}+E_{\mathrm{icc}}
$$

where $E_{\text {pot }}$ is a correction to account for potential alignment mismatch, and $E_{\mathrm{bf}}$ and $E_{\mathrm{icc}}$ are corrections to account for finite supercell effects.

\section{Potential alignment correction}

As previously mentioned, in periodic density functional theory, the total energy of a system is calculated relative to the background electrostatic potential. When calculating charged defects, the removal or addition of an electron requires the introduction of a jellium background that neutralises the overall charge of the cell. Accordingly, 
there is a mismatch between the reference electrostatic potential of the charged defect compared to that of the neutral host supercell. A correction must therefore be applied to ensure the total energies of both systems are comparable. This is defined as

$$
E_{\text {pot }}^{X, q}=q\left[V_{\mathrm{r}}^{X, q}-V_{\mathrm{r}}^{\mathrm{H}}\right] \text {, }
$$

where $V_{\mathrm{r}}^{\mathrm{H}}$ is the potential at a reference point in the host and $V_{\mathrm{r}}^{X, q}$ is the potential at the same reference point in the defected supercell. In practise, a core level far from the defect site is chosen as the reference point.

\section{Band filling correction}

Due to finite supercell sizes, the introduction of a defect causes an impurity-impurity interaction resulting in the formation of a defect band. In an infinitely large supercell, the defect would instead result in a single defect eigenstate. If the defect band lies deep within the band gap it will likely remain localised and show minimal effect on the surrounding electronic structure. In contrast, if the band lies within or close to the band edges, any electrons or holes that are introduced will artificially occupy the valence or conduction bands according to a Fermi-Dirac distribution. A correction is therefore applied to account for the effects on the total energy of inadvertent band filling. ${ }^{106}$ For shallow donors the correction is calculated as

$$
E_{\mathrm{bf}}=-\sum_{j, \mathbf{k}} \Theta\left(\varepsilon_{j, \mathbf{k}}-\tilde{\varepsilon}_{\mathrm{cbm}}\right) w_{\mathbf{k}} \eta_{j, \mathbf{k}}\left(\varepsilon_{j, \mathbf{k}}-\tilde{\varepsilon}_{\mathrm{cbm}}\right)
$$

with the correction for shallow acceptors given as

$$
E_{\mathrm{bf}}=\sum_{j, \mathbf{k}} \Theta\left(\tilde{\varepsilon}_{\mathrm{vbm}}-\varepsilon_{j, \mathbf{k}}\right) w_{\mathbf{k}}\left(1-\eta_{j, \mathbf{k}}\right)\left(\varepsilon_{j, \mathbf{k}}-\tilde{\varepsilon}_{\mathrm{vbm}}\right),
$$

where $\varepsilon_{j, \mathbf{k}}$ are electronic eigenvalues from the defect calculation, $\tilde{\varepsilon}_{\mathrm{cbm}}$ and $\tilde{\varepsilon}_{\mathrm{vbm}}$ are the eigenvalues of the conduction band minimum and valence band maximum of the host supercell after the potential alignment is applied, $\Theta$ is the Heaviside step function, $w_{\mathbf{k}}$ is the $\mathbf{k}$-point weight, and $\eta_{j, \mathbf{k}}$ is the band occupation. 
Image-charge correction

Charged defects require a further correction due to the slow decay of the Coulomb interaction with distance. Generally, the size of the supercells used do not allow for this interaction to decay completely, resulting in the spurious electrostatic interaction between defect charge sites in neighbouring cells. ${ }^{107}$ An image-charge correction is introduced to restore the energy of the system to that of a charged defect in the dilute limit. While several forms of this correction exist, most are based on evaluating the potential between two point charges in a neutralising jellium - the Madelung energy - calculated as

$$
E_{\text {Madelung }}=-\frac{q^{2} \alpha}{2 L \varepsilon}
$$

where $q$ is the charge, $\alpha$ is the structure-dependent Madelung constant, $L$ is the distance between the charges, and $\varepsilon$ is the static dielectric constant. 108

In practice, the electron density of a charged defect rarely acts as a point charge due to some degree of delocalisation. To account for this, Makov and Payne ${ }^{108}$ introduced a third-order expression taking into account the quadrupole moment of the charge density, $Q$, as

$$
E_{\mathrm{icc}}^{\mathrm{MP}}=-\frac{q^{2} \alpha}{2 L \varepsilon}-\frac{2 \pi q Q}{3 \varepsilon L^{3}} .
$$

Subsequently, Lany and Zunger demonstrated that the second term of this expression scaled proportional to $q^{2} / L$, instead of the expected $q / L^{3}$, due to the effect of the dielectric screening on $Q .{ }^{109}$ Accordingly, they derived an approximate expression for the correction based on the shape factor of the cell, $c_{\mathrm{sh}}$, as

$$
E_{\mathrm{icc}}^{\mathrm{LZ}}=\left[1+c_{\mathrm{sh}}\left(1-\varepsilon^{-1}\right)\right] \frac{q^{2} \alpha}{2 L \varepsilon} .
$$

For cases where $\varepsilon$ is sufficiently large and the supercell is isotropic as is the case for all materials studied in this thesis - the pre-factor can be further approximated as $2 / 3$. We have additionally used the formalism developed by Murphy et al., which takes into account any anisotropy in the dielectric screening. ${ }^{110}$ 


\section{Thermodynamic transition levels}

The presence of a defect often introduces defect states into the band gap of a material. These can be measured experimentally through techniques such as deep-level transient spectroscopy and are used to assess the nature of the defect. These states do not correspond to the eigenvalues of the Kohn-Sham wavefunctions obtained directly from the calculation on the defected system. Instead, the states of interest generally involve the transition from one defect charge state to another - resulting in the release or capture of an electron or hole.

The energy at which the charge state of a defect spontaneously transforms from $q \leftrightarrow q^{\prime}$ is termed the thermodynamic transition level, and is calculated as

$$
\varepsilon\left(X, q / q^{\prime}\right)=\frac{\Delta_{\mathrm{f}} H^{X, q}-\Delta_{\mathrm{f}} H^{X, q^{\prime}}}{q^{\prime}-q} .
$$

The position of the thermodynamic transition level relative to the valence and conduction band edges indicates whether a defect will contribute to conductivity or act as a charge trap. A defect state is termed shallow if it is within $k_{\mathrm{B}} T$ of the band edges. In particular, shallow donor defects near the conduction band minimum facilitate $n$-type conductivity, whereas shallow acceptor defects close to the valence band maximum promote $p$-type conductivity.

\section{Chemical potential limits}

The chemical potentials required to calculate the defect formation energy reflect the chemical reservoirs for the species involved in the defect. In practice, these reservoirs are controlled by the experimental growth conditions. Accordingly, by varying the chemical potentials one can explore a range of different chemical environments. The range of accessible chemical potentials is limited by the formation of secondary phases. As such, one must also calculate the total energy of all competing phases to ensure the set of chemical potentials remains experimentally achievable.

The chemical potentials are calculated by imposing a series of 
bounds. To illustrate this process we take GaAs as an example. Initially, the chemical potentials of gallium and arsenic are related by the stability of the GaAs phase, namely

$$
\mu_{\mathrm{Ga}}+\mu_{\mathrm{As}}=\mu_{\mathrm{GaAs}}=\Delta_{\mathrm{f}} H^{\mathrm{GaAs}} .
$$

The chemical potentials are limited by the formation of elemental gallium and arsenic as

$$
\begin{aligned}
& \mu_{\mathrm{Ga}} \leq \mu_{\mathrm{Ga}}^{\text {elemental }}, \\
& \mu_{\mathrm{As}} \leq \mu_{\mathrm{As}}^{\text {elemental }},
\end{aligned}
$$

where

$$
\begin{aligned}
& \mu_{\mathrm{Ga}}^{\text {elemental }}=\Delta_{\mathrm{f}} H^{\mathrm{Ga}}=0, \\
& \mu_{\mathrm{As}}^{\text {elemental }}=\Delta_{\mathrm{f}} H^{\mathrm{As}}=0 .
\end{aligned}
$$

In addition, the lower limit of $\mu_{\mathrm{Ga}}$, indicating a gallium poor environment, is dictated by the formation of elemental arsenic - e.g. by setting $\mu_{\mathrm{As}}=0$ in equation 3.18. Analogously, $\mu_{\mathrm{As}}$ is limited by the formation of elemental gallium. Together this gives

$$
\begin{gathered}
\mu_{\mathrm{Ga}} \geq \Delta_{\mathrm{f}} H^{\mathrm{GaAs}}, \\
\mu_{\mathrm{As}} \geq \Delta_{\mathrm{f}} H^{\mathrm{GaAs}} .
\end{gathered}
$$

Solving this set of simultaneous equations gives the range of thermodynamically accessible chemical potential environments. The CPLAP code was employed to calculate the chemical potential limits of the ternary systems studied in this thesis. ${ }^{111}$

\section{Transition level diagrams}

The defect properties of a system are commonly presented in a transition level diagram in which the defect formation energies are plotted as a function of Fermi level, at a fixed set of chemical potentials. Accordingly, information regarding both the thermodynamic cost and electronic behaviour of a defect is conveyed simultaneously. 
The transition level diagram for multiple chemical potential environments are often displayed together to indicate how the defect behaviour varies with experimental growth conditions. While a defect may possess many charge states, only the most stable charge state at each Fermi level is indicated. For example, in the case of the anion vacancy shown in Figure 3.1a, the +1 charge state is higher in energy than the neutral and +2 charge states at all Fermi levels and will therefore not be seen experimentally.

In these diagrams, the slope of the line indicates the charge state of the defect, with donor defects - i.e. those donating one or more electrons to the system - sloping upwards from left to right and acceptor defects - those donating one or more holes - sloping downwards. Neutral defects are indicated by horizontal lines, as their formation energies do not depend on the position of the Fermi level. The transition levels - also termed ionisation levels - are indicated by filled circles. The Fermi level is pinned roughly at the position where the lowest energy donor and acceptor defects cross, as indicated in Figure 3.1b. Accordingly, as the chemical potentials are adjusted the position of the Fermi level can be tuned.

\section{Self-consistent Fermi level}

While the position of the Fermi level can be estimated based on visual inspection of the transition level diagram as described above, in practice $E_{\mathrm{F}}$ will depend on multiple factors. These include the density of states and temperature of the sample. The Fermi level can be obtained in a self-consistent manner based on a set of thermodynamic transition levels, given the condition of charge neutrality ${ }^{112}$

$$
n_{0}+\sum_{i}\left|q_{i}\right| N_{q_{i}}^{\mathrm{A}}=p_{0}+\sum_{j}\left|q_{j}\right| N_{q_{j}}^{\mathrm{D}}
$$

where $n_{0}$ and $p_{0}$ are the concentration of electrons and holes respectively, $N_{q_{i}}^{\mathrm{A}}$ is the concentration of acceptor defects in charge state $q_{i}$, and $N_{q_{j}}^{\mathrm{D}}$ is the concentration of donor defects in charge state $q_{j}$. These
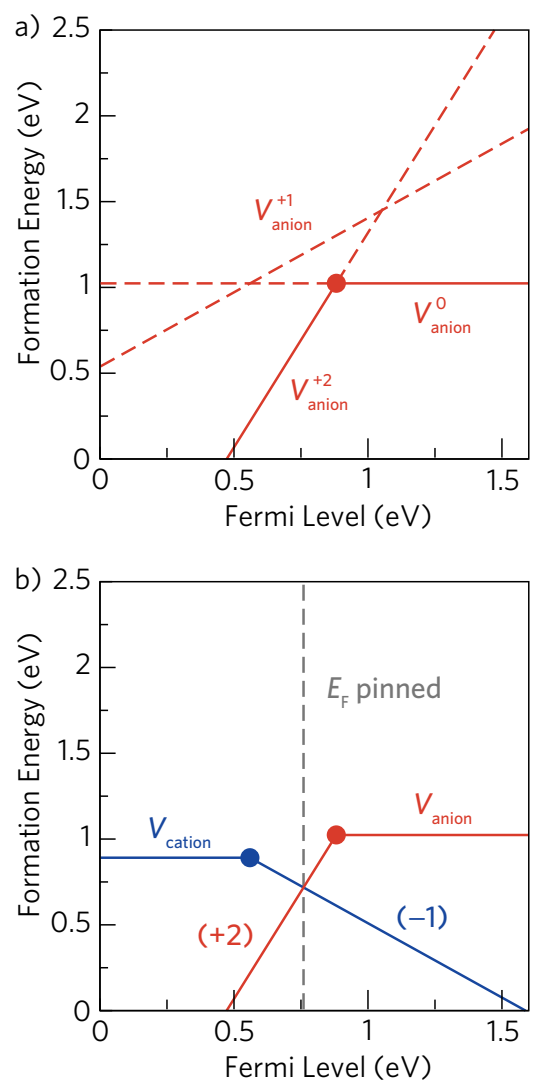

FIGURE 3.1: Example transition level diagrams for a hypothetical material. A) Only the lowest energy charge state at each Fermi level of the anion vacancy is indicated by the solid red line. B) The Fermi level is pinned near where the lowest energy donor and acceptor defects cross. 
concentrations depend on $E_{\mathrm{F}}$ as

$$
\begin{aligned}
N^{X, q} & =N_{0} \exp \left[\frac{\Delta_{\mathrm{f}} H^{X, q}\left(E_{\mathrm{F}}\right)}{k_{\mathrm{B}} T}\right], \\
n_{0} & =\int_{0}^{\infty} \frac{1}{\mathrm{e}^{\left(E-E_{\mathrm{F}}\right) / k_{\mathrm{B}} T}+1} g(E) \mathrm{d} E, \\
p_{0} & =\int_{0}^{\infty}\left[1-\frac{1}{\mathrm{e}^{\left(E-E_{\mathrm{F}}\right) / k_{\mathrm{B}} T}+1}\right] g(E) \mathrm{d} E,
\end{aligned}
$$

where $N_{0}$ is the density of sites where defect $X$ can form and $g(E)$ is the bulk density of states. Accordingly, the occupation of the valence and conduction bands (e.g. concentrations of holes and electrons) is determined by Fermi-Dirac statistics. In this thesis, the self-consistent Fermi level was calculated using the SC-FERMI code developed by Buckeridge et al. ${ }^{113-115}$

\section{Shockley-Read-Hall recombination rate}

The defect transition levels also play a role in determining the rate of Shockley-Read-Hall (trap-assisted) recombination. ${ }^{116}$ Here, the rate of recombination is determined by quasi-equilibrium semiconductor statistics. Crucially, in steady state the recombination rate of electrons and holes must be equal. Within this model, the recombination rate, $R^{\mathrm{SRH}}$, is calculated as

$$
R^{\mathrm{SRH}}=\frac{(n+\Delta n)(p+\Delta p)-n_{\mathrm{i}}^{2}}{\tau_{\mathrm{n}}\left(n+\Delta n+n_{1}\right)+\tau_{\mathrm{p}}\left(p+\Delta p+p_{1}\right)},
$$

where $p$ and $n$ are the self-consistent hole and electron concentrations, $\Delta p$ and $\Delta n$ are the change in hole and electron concentrations resulting from steady-state illumination and were set as $1 \times 10^{14} \mathrm{~cm}^{-3}$, and $n_{\mathrm{i}}$ is the concentration of electrons in an intrinsic sample. $\tau_{\mathrm{p}}$ $\left(\tau_{\mathrm{n}}\right)$ is the lifetime of holes (electrons) in the limit that all recombination centres are occupied with electrons (holes), also termed the Shockley-Read-Hall lifetime. The lifetimes are calculated as

$$
\tau=1 / N_{X} v \sigma
$$


where $N_{X}$ is the concentration of the defect, $v$ is the group velocity, and $\sigma$ is the defect capture cross-section. Due to the immense computational difficulty in calculating capture cross-sections we have assumed a constant value for all defects.

Returning to equation $3.29, p_{1}$ and $n_{1}$ indicate the concentration of holes and electrons if the Fermi level is pinned at the same energy as the defect transition level, $\varepsilon_{X}$. These are calculated according to Fermi-Dirac statistics as

$$
\begin{aligned}
& p_{1}=N_{\mathrm{v}} \exp \left[\frac{-\varepsilon_{X}-\varepsilon_{\mathrm{vbm}}}{k_{\mathrm{B}} T}\right], \\
& n_{1}=N_{\mathrm{c}} \exp \left[\frac{\varepsilon_{X}-\varepsilon_{\mathrm{cbm}}}{k_{\mathrm{B}} T}\right], \\
& n_{\mathrm{i}}^{2}=n_{1} \times p_{1}
\end{aligned}
$$

where $N_{\mathrm{v}}$ and $N_{\mathrm{c}}$ are the effective density of states of the valence and conduction bands, respectively, and are defined as

$$
\begin{aligned}
& N_{\mathrm{v}}=2\left(\frac{m_{\mathrm{h}}^{\star} k_{\mathrm{B}} T}{2 \pi \hbar^{2}}\right)^{3 / 2}, \\
& N_{\mathrm{c}}=2\left(\frac{m_{\mathrm{e}}^{\star} k_{\mathrm{B}} T}{2 \pi \hbar^{2}}\right)^{3 / 2} .
\end{aligned}
$$

\section{Vienna Ab initio Simulation Package}

All calculations in this thesis were carried out in the framework of density functional theory, using the Vienna $A b$ initio Simulation package (VASP). ${ }^{117-120}$ VASP is a periodic plane wave code, employing the projector augmented-wave method to describe the interactions between core and valence electrons. ${ }^{121}$ Due the complete set of PAW pseudopotentials included in the distribution and the high level of parallelisability, VASP is employed widely among the computational chemistry and physics communities. The VESTA package was used to plot crystal structures and charge density isosurfaces directly from the output of VAsP calculations. ${ }^{122}$ 



\section{Part II}

\section{Perovskite-inspired absorbers}





\section{REVIEW: PEROVSKITE PHOTOVOLTAICS}

As previously discussed, $\mathrm{CH}_{3} \mathrm{NH}_{3} \mathrm{PbI}_{3}$ (MAPI) and the hybrid perovskites have recently emerged as a remarkably efficient class of solar absorbers. Unfortunately, MAPI suffers from several issues that limit its commercial viability. A primary issue is device hysteresis, whereby significant differences are observed for $J-V$ curves measured under forward and reverse bias. Planar MAPI devices containing thin mesoporous $\mathrm{TiO}_{2}$ and small grain sizes are particularly affected and show losses in fill factors and efficiencies of up to $33 \% .{ }^{123}$ The presence of hysteresis has been ascribed to high levels of ionic conduction. ${ }^{124,125}$ In particular, mobile methylammonium ${ }^{126}$ and iodine vacancies ${ }^{127}$ that can migrate throughout the absorber layer in response to the photogenerated voltage - are thought to generate an electric field that opposes that of the $p-n$ junction.

While recent device improvements have minimised the effect of hysteresis, ${ }^{128,129}$ the stability of the hybrid perovskites is a critical issue which presents a major roadblock in the move toward commercialisation. Stability is particularly crucial as device longevity is essential to reach energy payback times. ${ }^{130}$ The facile decomposition of MAPI in moisture has been known since the earliest hybrid perovskite cells were produced, ${ }^{131,132}$ with further studies detailing the hydrolysation of MAPI upon contact with air. ${ }^{133-135}$ The thermal stability of MAPI is also poor, with decomposition occurring at temperatures above $85^{\circ} \mathrm{C} .136,137$ Recently, several studies, both theoretical and experimental, have indicated that MAPI is intrinsically unstable with respect to phase separation into $\mathrm{PbI}_{2}$ and $\mathrm{CH}_{3} \mathrm{NH}_{3} \mathrm{I}_{3}$ due to its 


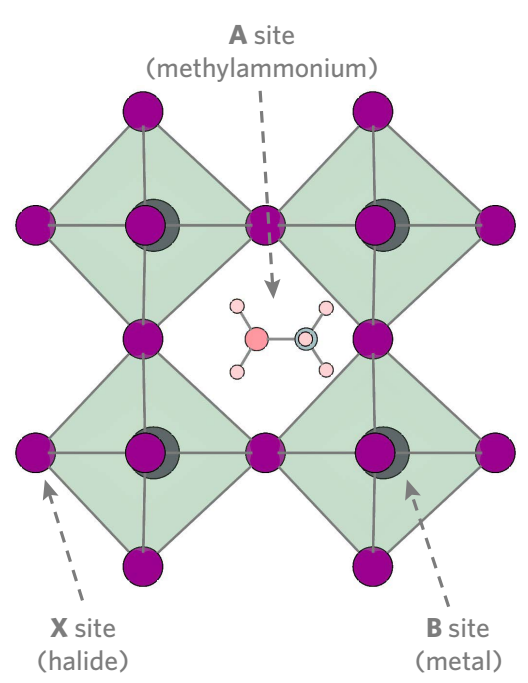

FIGURE 4.1: Schematic of perovskite structure indicating the $A$, $B$, and $X$ lattice sites. small formation energy. ${ }^{138-141}$ As such, modifications to the MAPI structure that confer greater stability are highly desirable. ${ }^{142}$

\section{Perovskite structure}

MAPI adopts the perovskite $\mathrm{ABX}_{3}$ structure (where $\mathrm{A}=\mathrm{CH}_{3} \mathrm{NH}_{3}, \mathrm{~B}$ $=\mathrm{Pb}$, and $\mathrm{X}=\mathrm{I}$ ), where corner sharing $\mathrm{PbI}_{6}$ octahedra form a cage enclosing the organic cation (Fig. 4.1). One route to tuning the properties of MAPI is through changing the non-bonding organic component. To date, after over nine years of development, the only organic cations to be successfully incorporated into the perovskite motif are methylammonium (MA) and formamidinium (FA). Incorporation of larger cations results in reduced dimensionality structures due to disruption of the three-dimensional (3D) lead iodide cage. ${ }^{143-145}$ Introducing FA in the place of MA to form $\mathrm{CH}\left(\mathrm{NH}_{2}\right)_{2} \mathrm{PbI}_{3}$ (FAPI), produces films with a slightly reduced band gap of $1.48 \mathrm{eV}$, improved PCEs, extended photoluminescence (PL) lifetimes, and greater thermal stability. ${ }^{146,147}$ Unfortunately, FAPI can also form in a thermally accessible (less than $360 \mathrm{~K}$ ) hexagonal $\delta$-phase possessing a large band gap, which cannibalises device performance. ${ }^{148,149}$ Including $20 \%$ MA in the synthesis of FAPI stabilises the black $\alpha$-phase whilst preserving long exciton lifetimes and high efficiencies, ${ }^{150,151}$ however, the longterm stability of these devices is yet to be addressed.

An alternative method for improving long-term stabilities is to completely replace the organic component, forming an all-inorganic perovskite. ${ }^{152,153}$ Eperon et al. recently produced a functioning $\mathrm{CsPbI}_{3}$ device through suppression of the formation of a competing nonperovskite yellow phase during synthesis. ${ }^{154,155}$ Despite remarkable thermal stability up to $300^{\circ} \mathrm{C}$, their films were highly sensitive to ambient conditions and performed poorly, reaching a PCE of only $2.9 \%$.

MAPI can also be tuned on the $\mathrm{X}$ site by replacing iodine with other halides. ${ }^{23}$ As the position of the valence band maximum is largely controlled by the binding energy of the halide valence $p$ orbitals, swapping I for $\mathrm{Br}$ and $\mathrm{Cl}$ results in materials with band gaps 
a) $\begin{array}{lllllllllll}X=1 & 0.9 & 0.8 & 0.7 & 0.6 & 0.5 & 0.4 & 0.3 & 0.2 & 0.1 & X=0\end{array}$

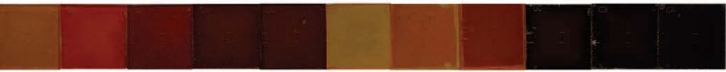

b)

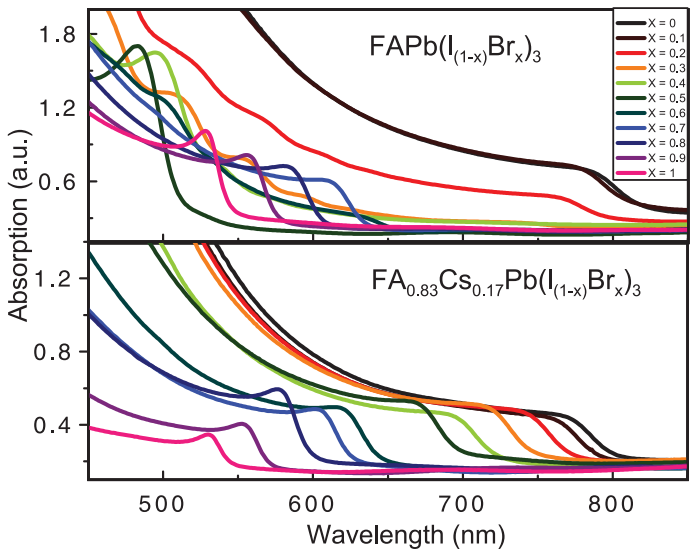

$\mathrm{FA}_{0.83} \mathrm{Cs}_{0.17} \mathrm{~Pb}\left[\mathrm{I}_{(1-x)} \mathrm{Br}_{x}\right]_{3}$ $\begin{array}{lllllllllll}\mathrm{X}=1 & 0.9 & 0.8 & 0.7 & 0.6 & 0.5 & 0.4 & 0.3 & 0.2 & 0.1 & \mathrm{X}=0\end{array}$

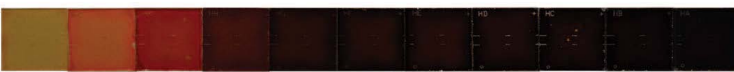

c)

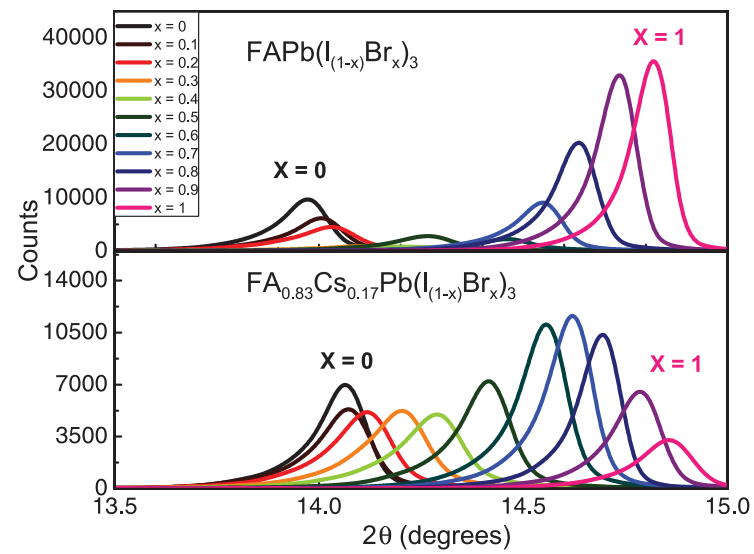

too large for photovoltaic applications. However, mixed-halide perovskites are commonly used to fine-tune the absorber properties. In the first perovskite cells, $\mathrm{PbCl}_{2}$ used as a precursor resulted in a small proportion of the chlorine being incorporated into the perovskite layer, in turn leading to more even distribution of nucleation sites and smoother films. ${ }^{128,156,157}$ Furthermore, use of bromide in a solid solution allows for an adjustable band gap ${ }^{158}$ and reduced levels of hysteresis. ${ }^{129}$ Rehman et al. trialled a mixed bromide/iodide absorber layer, $\mathrm{FAPb}\left(\mathrm{Br}_{x} \mathrm{I}_{1-x}\right)_{3}$, as a potential top cell in tandem devices. ${ }^{159}$ While the composition where $x=0.3-0.5$ allowed for an ideal top cell band gap of $\sim 1.7-1.8 \mathrm{eV}$, the resulting material appeared amorphous with weak optical absorption and dramatically reduced charge-carrier diffusion lengths. This observation has been confirmed by theoretical calculations on the related $\operatorname{MAPb}\left(\mathrm{Br}_{x} \mathrm{I}_{1-x}\right)_{3}$ system, with the region between $0.3<x<0.6$ showing intrinsic instability with respect to spinodal decomposition at $300 \mathrm{~K} .{ }^{160}$

McMeekin et al. have recently demonstrated a mixed-cation mixedhalide perovskite system which subjugates the aforementioned phase instability region through partial substitution of formamidinium with caesium (Fig. 4.2). ${ }^{161}$ With a composition of $\mathrm{FA}_{0.83} \mathrm{Cs}_{0.17} \mathrm{~Pb}\left(\mathrm{I}_{0.6} \mathrm{Br}_{0.4}\right)_{3}$, their highly crystalline films possessed a large $\mathrm{V}_{\mathrm{oc}}$ of $1.2 \mathrm{eV}$ and efficiencies up to $17.9 \%$. When employed as a top cell coupled with
FIGURE 4.2: (a) Photographs, (b) ultraviolet-visible absorbance spectra, and (c) X-ray diffraction patterns of $\mathrm{FAPb}\left[\mathrm{I}_{(1-x)} \mathrm{Br}_{x}\right]_{3}$ and $\mathrm{FA}_{0.83} \mathrm{Cs}_{0.17} \mathrm{~Pb}\left[\mathrm{I}_{(1-x)} \mathrm{Br}_{x}\right]_{3}$ perovskite films, with $\mathrm{Br}$ composition increasing from $x=0-1$. Adapted from ref. 161 
a crystalline silicon module in a tandem device, the same composition achieved an efficiency of $19.8 \%$. In 2017, inclusion of rubidium cations in a mixed-cation mixed-halide perovskite produced thin films with a band gap of $1.73 \mathrm{eV} .{ }^{162}$ When combined with an interdigitated back-contact silicon cell, devices reached an impressive efficiency of $26.4 \%$.

\section{Reduced dimensionality perovskites}

Over the last four years, layered perovskites have emerged as a promising route to increased stabilities. ${ }^{163,164}$ When large organic cations are incorporated in the synthesis of the hybrid perovskites, lower dimensionality structures are formed as the cations cannot fit within the perovskite cage. ${ }^{165,166}$ These structures, composed of layers of $\mathrm{PbI}_{6}$ octahedra "capped" by organic molecules, form two-dimensional (2D) or Ruddlesden-Popper like phases. ${ }^{167}$ The primary mode of stabilisation is through van der Waals interactions that occur between the capping molecules and the surface of the inorganic layer. ${ }^{168-171}$ Analogous to MAPI, the physical and electronic properties of the layered perovskites can be tuned by modifying the organic, metal and halide components. ${ }^{143,144}$ The success of the layered perovskites is partially due to the ability to control the thickness of the inorganic layer. By tailoring the stoichiometric quantities of the organic versus inorganic components, the number of layers of perovskite octahedra per slab $(n)$, for the series (PEA) $)_{2}\left(\mathrm{CH}_{3} \mathrm{NH}_{3}\right)_{n-1} \mathrm{~Pb}_{n} \mathrm{I}_{3 n+1}$ (where PEA $\left.=\mathrm{C}_{6} \mathrm{H}_{5}\left(\mathrm{CH}_{2}\right)_{2} \mathrm{NH}_{3}{ }^{+}\right)$, can be regulated. ${ }^{172}$ In this scheme, the limit $n=\infty$ corresponds to the $3 \mathrm{D}$ perovskite structure, $n=1$ denotes the 2D layered structure, and $n>1$ describes "quasi-2D" systems (Fig. 4.3).

In the 1990s, Mitzi et al. characterised a series of intercalated organic-inorganic germanium, tin, and lead perovskites, noting the role of large organic cations in promoting enhanced thermal and chemical stability. properties. ${ }^{167-169,172-178}$ It was shown that moving from a $3 \mathrm{D}$ to $2 \mathrm{D}$ perovskite structure widened the optical band gap considerably, with concomitant reduction in $n$-type conductivity. This 

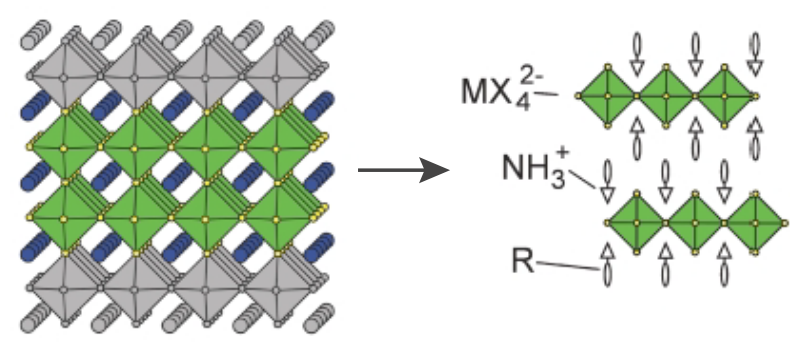

$$
n=1
$$

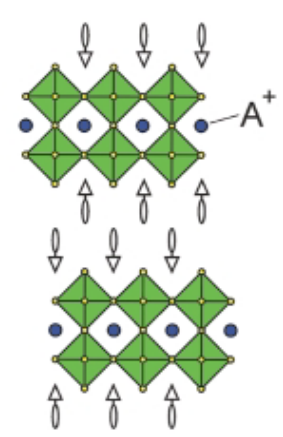

$n=2$

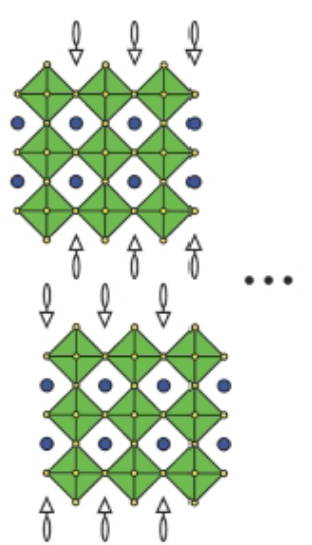

$n=3$

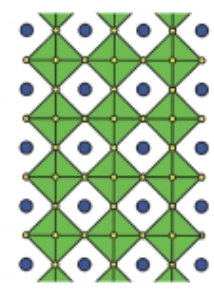

$n=\infty$

trend was attributed, in part, to the presence of high exciton binding energies - often greater than $300 \mathrm{eV}^{175,179}$ — which emerged due to spatial constraints and dielectric mismatch between the inorganic and organic components. ${ }^{180,181}$ Such properties are undesirable in photovoltaic absorbers as they result in a reduced $V_{\text {oc }}$ that negatively impacts PCEs. Conversely, stability is greatest for the $2 \mathrm{D} n=1$ structure and decreases as the thickness of the perovskite layer is increased. Clearly, the primary challenge in designing novel layered perovskites is to optimise stoichiometry such that stability is balanced against optoelectronic performance.

In an attempt to offset the large band gap seen when adopting the $2 \mathrm{D}$ perovskite motif, the first layered perovskites employed in solar cells attempted to tune the electronic properties through shrewd choice of organic cation. Smith et al. synthesised an $n=3$ perovskite containing the bulky $\mathrm{C}_{6} \mathrm{H}_{5}\left(\mathrm{CH}_{2}\right)_{2} \mathrm{NH}_{3}{ }^{+}$(PEA) cation, enabling a significant reduction in exciton binding energies $(40 \mathrm{meV})$ but a band gap outside the ideal range $(2.1 \mathrm{eV}) .{ }^{182}$ Despite this, their cells performed reasonably well, recording efficiencies up to $4.73 \%$, and displayed impressive stabilities, with little degradation seen after 40 days in air with $52 \%$ humidity. Similarly, $n=3$ devices containing another large organic cation, $\mathrm{CH}_{3}\left(\mathrm{CH}_{2}\right)_{3} \mathrm{NH}_{3}{ }^{+}$, were synthesised by Cao et al. through a low-cost spin-coating process, achieving efficiencies of $4.02 \%{ }^{183}$

Both the devices produced by Smith et al. and Cao et al. suf-

FIGURE 4.3: Schematic demonstrating the relationship between the three-dimensional perovskites $(n=\infty)$ and their layered counterparts $(n \geq 1)$. Adapted from ref. 168. 
fered from small charge-carrier diffusion lengths that severely impacted efficiencies. ${ }^{182,183}$ Recently, Quan et al. have produced the first hysteresis-free planar perovskite solar cell, based on a 2D absorbing layer containing PEA. ${ }^{170}$ Density functional theory calculations were employed in tandem with complementary experimental studies on electronic and physical properties, to optimise the thickness of the perovskite layer to $40<n<60$. Van der Waals forces between the organic and perovskite layers were found to reduce the desorption rate of $\mathrm{CH}_{3} \mathrm{NH}_{3} \mathrm{I}(\mathrm{MAI})$ - the primary decomposition route of MAPI ${ }^{88,184,185}$ - by six orders of magnitude. Furthermore, these quasi-2D devices successfully combined the ideal optoelectronic properties of MAPI with the stability of 2D perovskites, enabling an efficiency of $15.3 \%$ for the composition where $n=60 .{ }^{170}$

\section{Tin-based perovskites}

The instability of lead-based perovskite photovoltaics has raised concerns over the environmental effects if toxic lead were to leach into the environment. ${ }^{136}$ Under EU law, commercial solar cells are exempt from the Restriction of Hazardous Substances Directive and are not regulated based on their lead content. ${ }^{186}$ To ensure minimal effect on the environment, however, devices will require thorough encapsulation, which will increase the cost of manufacture. The last few years have therefore seen many attempts to produce lead-free perovskite photovoltaics. Tin is of particular interest due to its low cost and minimal toxicity. ${ }^{187}$ Furthermore, it is isoelectric with lead and is therefore expected to show many of the same properties.

Unfortunately, the relatively facile oxidation of $\mathrm{Sn}^{2+}$ to $\mathrm{Sn}^{4+}$ has limited the adoption and development of tin hybrid perovskites. ${ }^{188,189}$ In 2015, Koh et al. demonstrated that by using small amounts of $\mathrm{SnF}_{2}$ in the synthesis of formamidinium tin iodide $\left(\mathrm{HC}\left(\mathrm{NH}_{2}\right)_{2} \mathrm{SnI}_{3}, \mathrm{FASI}\right)$ the reduction of $\mathrm{Sn}^{4+}$ to $\mathrm{Sn}^{2+}$ was promoted. ${ }^{190}$ While this greater stability occurs at the expense of conductivity, this technique enabled the fabrication of FASI-based devices with efficiencies of $2.1 \%$. FASI possesses a band gap of $1.4 \mathrm{eV}-$ in the ideal range for a solar ab- 
sorber - and, in contrast to its lead analogues, does not show other thermally accessible phases that might negatively impact real-world efficiencies. ${ }^{148,151,191}$ Further work extended this method by incorporating pyrazine to prevent surface segregation of the $\mathrm{SnF}_{2} \cdot{ }^{192}$ This enabled improved power conversion efficiencies up to $4.8 \%$ (Fig. 4.4), with devices showing limited loss in performance when kept in ambient conditions for 100 days. More recently, FASI-based devices containing very small concentrations of a $2 \mathrm{D}$ tin-based perovskite have demonstrated efficiencies of $9.0 \% .{ }^{193}$ These devices showed much greater stability in light and ambient conditions compared to films prepared in an analogous manner but instead using $\mathrm{SnF}_{2}$.

The all-inorganic $\mathrm{CsSnI}_{3}$ has also faced issues due to the undesired oxidation of $\mathrm{Sn}$. Originally employed as a solid-state hole transporting layer for dye-sensitised solar cells, ${ }^{194} \mathrm{CsSnI}_{3}$ possesses a band gap of $1.30 \mathrm{eV}$ and intrinsic $p$-type conductivity. ${ }^{155,196}$ While initial devices saw very poor performance due to the polycrystalline nature of the thin films, ${ }^{197}$ subsequent work employing $\mathrm{SnF}_{2}$ as an additive enabled efficiencies up to $2.0 \% .{ }^{198,199}$ Unfortunately, performance remains limited by poor open-circuit voltages of $0.24 \mathrm{~V}$, about four times smaller than seen in MAPI-based devices. ${ }^{200,201} \mathrm{CsSnI}_{3}$-based devices face further challenges to due the formation of an alternate wide band gap secondary phase upon exposure to air or organic solvents. ${ }^{195,202,203}$ Accordingly, $\mathrm{CsSnI}_{3}$-based devices will require significant development if they are to become practically relevant.

When moving from $\mathrm{CH}_{3} \mathrm{NH}_{3} \mathrm{PbI}_{3}$ to $\mathrm{CH}_{3} \mathrm{NH}_{3} \mathrm{SnI}_{3}$, the band gap is reduced from $1.55 \mathrm{eV}$ to $1.20 \mathrm{eV} .{ }^{188} \mathrm{~A}$ similar trend is seen for the tin layered perovskites. For example, in the butylammonium (BA) layered perovskite series $(\mathrm{BA})_{2}(\mathrm{MA})_{n-1} \mathrm{~Pb}_{n} \mathrm{I}_{3 n+1}$, the band gaps for the compositions where $n=1,2$, and 3 , are $2.43 \mathrm{eV}, 2.17 \mathrm{eV}$, and $2.03 \mathrm{eV}$, respectively. ${ }^{183}$ Moving to their tin analogues, the band gaps for the same $n$ members were recently measured as $1.83 \mathrm{eV}, 1.64 \mathrm{eV}$, and $1.50 \mathrm{eV}$, respectively. ${ }^{204}$ Accordingly, the band gaps for the tin series are considerably closer to the ideal dictated by the ShockleyQuiesser limit.

In 2017, the compositions of $(\mathrm{BA})_{2}(\mathrm{MA})_{n-1} \mathrm{Sn}_{n} \mathrm{I}_{3 n+1}$ where $n=3$

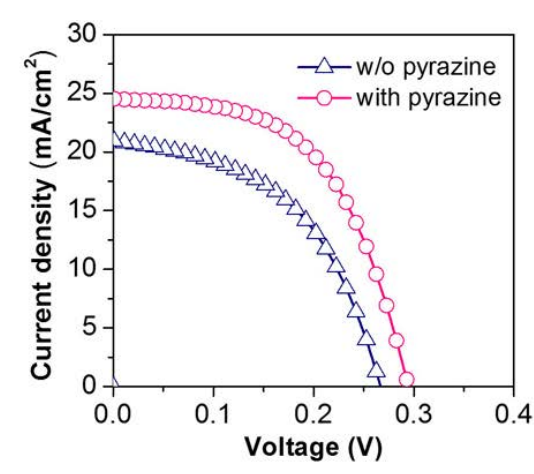

FIGURE 4.4: $J-V$ curves demonstrating the effect of pyrazine on $\mathrm{FASnl}_{3}$ devices. Taken from ref. 192 
and 4 were incorporated into photovoltaic devices containing mesoporous $\mathrm{TiO}_{2} \cdot{ }^{204}$ Using dimethyl sulfoxide (DMSO) as a solvent, the perovskite layer was grown such that the $2 \mathrm{D}$ sheets were vertically aligned perpendicular to the electrodes. This arrangement has been shown to be required for maximising the efficiency of layered devices. Despite this, the cells performed relatively poorly, achieving efficiencies up to $1.94 \%$ and $2.53 \%$ for the $n=3$ and 4 compounds, respectively. Promisingly, however, the stability of the devices was dramatically improved, with the cells retaining $90 \%$ of their peak performance after one month. In contrast, similarly prepared $\mathrm{CH}_{3} \mathrm{NH}_{3} \mathrm{SnI}_{3}$ devices showed $0 \%$ efficiency after this time. Subsequent work on $(\mathrm{PEA})_{2}(\mathrm{FA})_{8} \mathrm{Sn}_{9} \mathrm{I}_{29}$ where the PEA cation is phenylethylammonium, enabled device efficiencies up to $5.94 \% .{ }^{205}$ Again, judicious choice of solvent was employed to ensure vertically aligned growth. Furthermore, the resulting thin films were shown to possess significantly improved stability compared to $\mathrm{FASnI}_{3}$. While still very much in development, the progress seen in layered tin-based perovskites over the last few months provides hope for the future of lead-free perovskite photovoltaics.

\section{Outlook}

The success of the hybrid perovskites is evident. That a little-known and under-studied material can achieve power conversion efficiencies over $22 \%$ in less than ten years of development is remarkable. Despite this, there remain several key issues that must be addressed if perovskite-based photovoltaics are to be successfully commercialised. Crucially, their long-term stability presents a major roadblock, which, even after significant research attention, still plagues the perovskite community. The recent emergence of the layered perovskites provides a glimmer of hope. In particular, "quasi-layered" materials combine the dramatic increase in stability of the purely layered structures with the optimal optoelectronic properties possessed by the threedimensional perovskites. Accordingly, further work tuning layer thickness may prove incredibly fruitful. 
While currently the use of lead in photovoltaic devices is not regulated, this may not remain the case in the future. Despite the isoelectronic nature of the group 14 metals, tin-based perovskite absorbers cannot yet match the performance of their lead counterparts. Here, progress has been hampered by the sensitivity of $\mathrm{Sn}^{2+}$ to oxidation, which complicates device fabrication and stability. Recent months have seen significant improvements in this area, particularly for FASI-based devices, which have now achieved efficiencies of $9.0 \%$. Regardless, it is essential that alternative schemes for improving the stability of these materials be developed. 



\section{PSEUDOHALIDE PEROVSKITE}

\section{A B SORBERS}

\section{Introduction}

The substitution of iodine for thiocyanate (SCN) has recently emerged as a novel route to increasing the stability of MAPI-based devices. ${ }^{141,206-209}$ In 2015, Chen et al. showed that inclusion of $\mathrm{SCN}^{-}$, a pseudohalide with an ionic radius similar to that of $\mathrm{I}^{-}(217 \mathrm{pm} v s 220 \mathrm{pm}),{ }^{210}$ to form $\mathrm{CH}_{3} \mathrm{NH}_{3} \mathrm{PbI}_{3-x} \mathrm{SCN}_{x}$ promoted larger crystal domains with fewer trap sites than in undoped MAPI samples. ${ }^{206}$ Their devices achieved efficiencies up to $11 \%$ at an optimum level of $5 \%$ SCN incorporation and possessed enhanced stability, greater reproducibility, and reduced levels of hysteresis than comparable MAPI films. ${ }^{123,211}$ The effect of SCN as a dopant in MAPI was investigated by Halder et al., who observed a ten-fold enhancement in the intensity of the photoluminescence response, coupled with a band gap widening of $8 \mathrm{meV} .{ }^{207}$ Jiang et al. provided the first structural characterisation of pseudohalide containing perovskites, suggesting that $\mathrm{CH}_{3} \mathrm{NH}_{3} \mathrm{~Pb}(\mathrm{SCN})_{2} \mathrm{I}$ films crystallised in the cubic perovskite structure. ${ }^{208}$ Their films, with a band gap of $1.53 \mathrm{eV}$, were significantly more stable in air with 95\% humidity than pure MAPI, the reason for which was not elucidated. ${ }^{138}$ Devices containing $\mathrm{CH}_{3} \mathrm{NH}_{3} \mathrm{~Pb}(\mathrm{SCN})_{2} \mathrm{I}$ films showed an efficiency of $8.3 \%$, with a greater $\mathrm{V}_{\text {oc }}(0.87 \mathrm{eV} v s 0.80 \mathrm{eV})$ but smaller fill factor (FF, $52 v s$ 63) than similarly prepared MAPI films. ${ }^{208} \mathrm{Un}$ fortunately, this was the first report of SCN incorporation on the X site in the cubic perovskite structure, leading to doubts of the structural characterisation within the hybrid-perovskite community. 
FIGURE 5.1: Crystal structure of $\left(\mathrm{CH}_{3} \mathrm{NH}_{3}\right)_{2} \mathrm{~Pb}(\mathrm{SCN})_{2} \mathrm{I}_{2}$ viewed along the [001] direction. $\mathrm{Pb}$, $\mathrm{I}, \mathrm{S}, \mathrm{C}, \mathrm{N}$, and $\mathrm{H}$ atoms denoted by dark grey, purple, yellow, red, blue, and pink spheres, respectively. The octahedral nature of $\mathrm{Pb}$ is illustrated using green polyhedra.

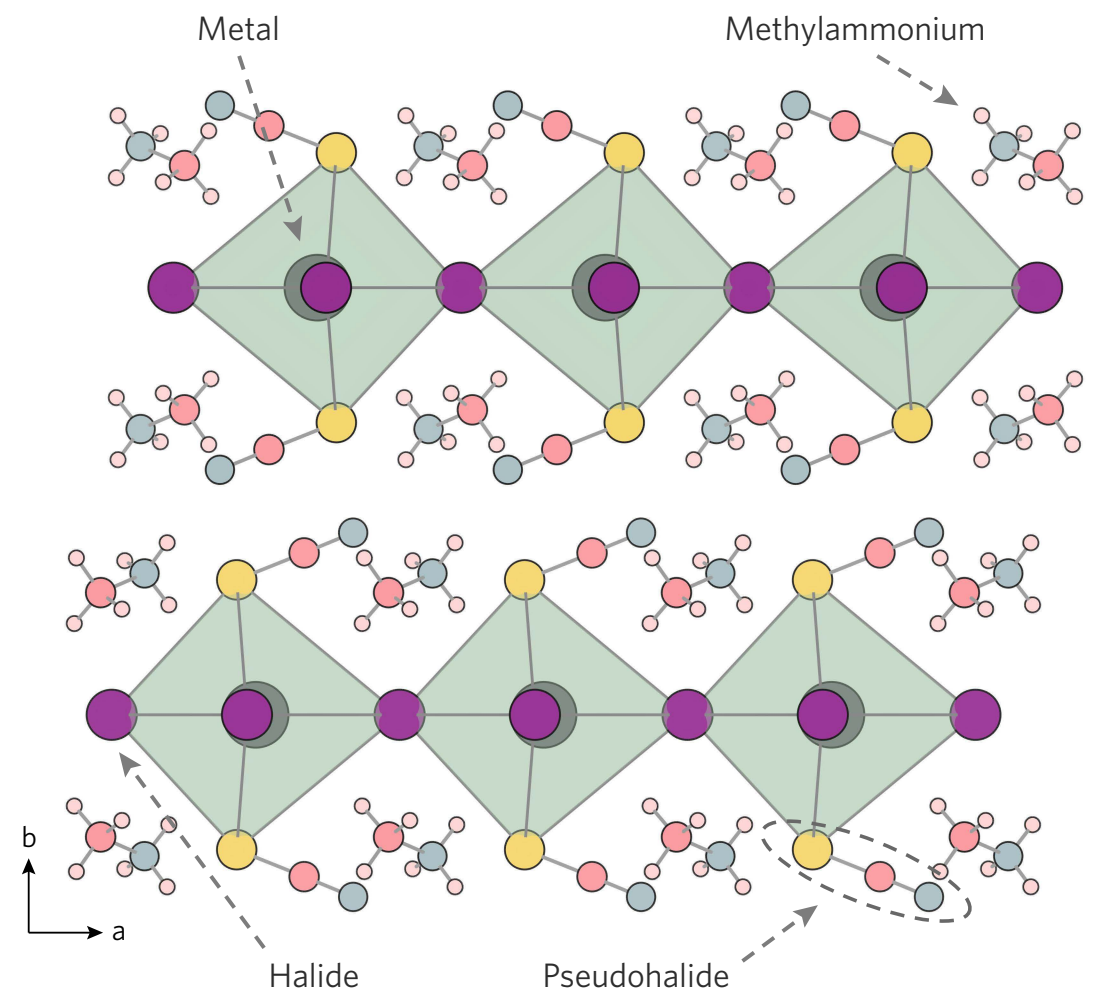

Subsequently, Daub and Hillebrecht showed that the reaction of $\mathrm{Pb}(\mathrm{SCN})_{2}$ and $\mathrm{CH}_{3} \mathrm{NH}_{3} \mathrm{I}$ results in the formation of $\left(\mathrm{CH}_{3} \mathrm{NH}_{3}\right)_{2} \mathrm{~Pb}(\mathrm{SCN})_{2} \mathrm{I}_{2}$, which we will denote MAPSI for simplicity. ${ }^{209}$ MAPSI crystallises in a layered orthorhombic structure $\left(P n m 2_{1}\right)$, similar to the $2 \mathrm{D} n=1$ perovskites, with $\mathrm{Pb}$ octahedrally coordinated to two axial SCN and four equatorial I, with the methylammonium sandwiched between the layers (Fig. 5.1). Crucially, the authors demonstrated that the Xray diffraction (XRD) pattern for MAPSI matched the XRD pattern of the $\mathrm{CH}_{3} \mathrm{NH}_{3} \mathrm{~Pb}(\mathrm{SCN})_{2} \mathrm{I}$ films produced by Jiang et al. ${ }^{208}$

There has been some debate as to the magnitude of MAPSI's optical band gap: initial work into thiocyanate incorporation indicated a direct optical gap of $1.57 \mathrm{eV},{ }^{209,212}$ however, a study performed by Xiao et al. reported thin films with indirect and direct gaps of $2.04 \mathrm{eV}$ and $2.11 \mathrm{eV}$, respectively. ${ }^{213}$ Recently, Umeyama et al. have suggested MAPSI possesses a larger band gap than originally thought, noticing a red-to-black piezochromic response upon compression $(2.6 \mathrm{GPa}){ }^{214}$ While the cause of this inconsistency is currently unknown, there have been suggestions that contamination of MAPSI with slight amounts of MAPI may play a role. An alternative suggestion, proposed by 
Younts et al., relies on the observation of highly efficient triplet state formation in MAPSI thin films. ${ }^{215}$ They report a triplet energy of $1.64 \mathrm{eV}$, with phosphorescence over 47 times more intense than the band gap fluorescence, thereby explaining some of the previous photoluminescence results that have frustrated band gap determination. Despite this, a band gap of $\sim 2.1 \mathrm{eV}$ and exciton binding energies less than $200 \mathrm{meV}^{214}$ are uncommon in two-dimensional perovskites, which often show band gaps greater than $2.7 \mathrm{eV}$ with $350 \mathrm{meV}$ exciton binding energies. ${ }^{216}$ As such, MAPSI presents an intriguing material that deserves further study.

\section{Methodology}

Calculations were performed using the Vienna $A b$ initio Simulation Package. A k-point mesh of $\Gamma$-centred $1 \times 4 \times 4$ and plane wave cutoff of $400 \mathrm{eV}$ was found to converge the 50 atom unit cell of $\left(\mathrm{CH}_{3} \mathrm{NH}_{3}\right)_{2} \mathrm{~Pb}(\mathrm{SCN})_{2} \mathrm{I}_{2}$ and all analogues to within $1 \mathrm{meV} /$ atom. During geometry optimisations, the cutoff was increased to $520 \mathrm{eV}$ to avoid errors resulting from Pulay stress. ${ }^{85}$ The structures were deemed converged when the forces totalled less than $10 \mathrm{meV} \AA^{-1}$.

Several functionals were used in this work: For geometry relaxations, PBEsol and PBE were employed, with and without the addition of Grimme's D3 dispersion correction. Electronic properties were calculated using HSE06 with the addition of spin-orbit coupling effects (HSE06+SOC). The Brillouin zone for the Pnm $2_{1}$ space group, indicating the high-symmetry points explored in the band structure, is provided in Fig. 5.2. Density functional perturbation theory (DFPT) was employed, in combination with the PBEsol functional, to calculate the ionic contribution to the dielectric constants, with a denser $\mathbf{k}$-point mesh of $\Gamma$-centred $3 \times 6 \times 6$ required to achieve convergence.

For band alignment calculations, the core-level alignment approach of Wei and Zunger was employed, ${ }^{217}$ using a slab model with $30 \AA$ of vacuum and a $25 \AA$ thick slab. The slab was cleaved along the non-polar (010) surface, due to the absence of any dangling bonds.

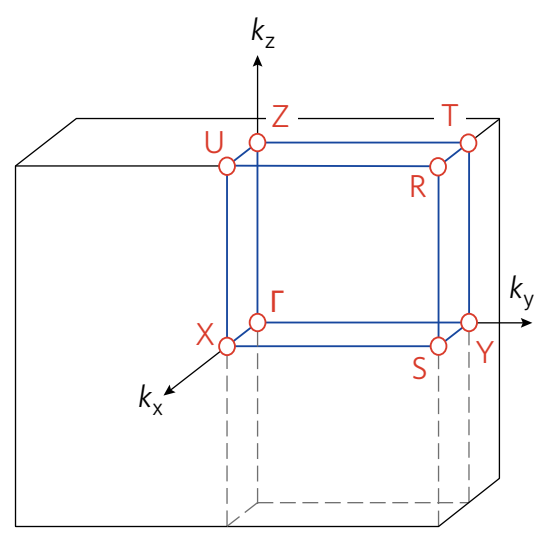

FIGURE 5.2: Brillouin zone of the $P n m 2_{1}$ space group. Coordinates of the high symmetry points used for the band structures and effective masses: $\Gamma=(0,0,0) ; Y$ $=(1 / 2,0,0) ; X=(0,1 / 2,0) ; Z=$ $(0,0,1 / 2) ; U=(0,1 / 2,1 / 2) ; T=$ $(1 / 2,0,1 / 2) ; S=(1 / 2,1 / 2,0) ; R=$ $(0,1 / 2,1 / 2)$. 
Due to the size of the model, which precluded the use of HSE06+SOC, band alignment calculations were performed using HSE06, with an explicit correction to the band gap and valence band maximum position taken from the HSE06+SOC calculated bulk.

Defect calculations were performed in a $1 \times 3 \times 3$ supercell containing 450 atoms, using the PBEsol functional and a $\Gamma$-centred $2 \times 2 \times 2$ k-point mesh. The defect energies were corrected to account for use of a finite-sized supercell, using the potential level alignment, bandfilling and image-charge corrections described in Chapter 3.

\section{Results}

\section{Geometric structure}

The crystal structure of MAPSI was minimised using three functionals, PBEsol, PBEsol with the addition of Grimme's D3 dispersion correction $(\mathrm{PBEsol}+\mathrm{D} 3)$ and $\mathrm{PBE}$ with the $\mathrm{D} 3$ correction $(\mathrm{PBE}+\mathrm{D} 3)$, with the results given in Table 5.1. Both PBEsol and PBE+D3 show good agreement with the experimental single crystal structure, ${ }^{209}$ with PBEsol+D3 severely underestimating the lattice constants. The $a$ lattice parameter is underestimated across every functional, suggesting that thermal effects may play a role in determining the interlayer separation. The PBEsol relaxed structure possessed lattice constants closest to experiment and was therefore used for all subsequent calculations.

TABLE 5.1: Lattice parameters of $\left(\mathrm{CH}_{3} \mathrm{NH}_{3}\right)_{2} \mathrm{~Pb}(\mathrm{SCN})_{2} \mathrm{I}_{2}$ calculated using PBEsol, PBEsol+D3 and $\mathrm{PBE}+\mathrm{D} 3$. Experimental error or percentage difference from experiment in parentheses. All cell angles were found to be $90^{\circ}$

\begin{tabular}{llll}
\hline & $a(\AA)$ & $b(\AA)$ & $c(\AA)$ \\
\hline PBEsol & $6.230(-0.59 \%)$ & $18.268(-1.68 \%)$ & $6.475(+0.14 \%)$ \\
PBEsol+D3 & $6.134(-2.12 \%)$ & $17.657(-4.97 \%)$ & $6.388(-1.21 \%)$ \\
PBE+D3 & $6.274(+0.11 \%)$ & $18.232(-1.87 \%)$ & $6.525(+0.91 \%)$ \\
\hline Experiment $^{209}$ & $6.267(7)$ & $18.580(2)$ & $6.466(6)$ \\
\hline
\end{tabular}

\section{Electronic properties}

Calculations on the electronic structure of MAPSI were performed using the HSE06 hybrid DFT functional, with explicit treatment of 
spin-orbit coupling (SOC), to ensure the accurate treatment of relativistic effects known to strongly affect the electronic structure of the lead iodide perovskites. The band gap of MAPSI was found to be $1.79 \mathrm{eV}$, significantly smaller than the reported experimental optical band gap of $\sim 2.1 \mathrm{eV} .{ }^{214,215}$ This underestimation of the band gap is similar to that seen in MAPI, where tuning of the amount of HartreeFock exchange, $\alpha$, is needed to adjust the calculated band gap to the experimental gap. In this work we have chosen not to artificially fit the band gap parameter as this discrepancy may arise from several factors, including the underestimation of the $a$ lattice parameter.

The projected density of states calculated by HSE06+SOC is shown in Figure 5.3a. The valence band maximum (VBM) is dominated by I $p$ states, with some contribution from $\mathrm{Pb} s$ states. Similar to elec-
FIGURE 5.3: Comparison of the electronic structures of $\left(\mathrm{CH}_{3} \mathrm{NH}_{3}\right)_{2} \mathrm{~Pb}(\mathrm{SCN})_{2} \mathrm{I}_{2}$ (MAPSI) and $\mathrm{CH}_{3} \mathrm{NH}_{3} \mathrm{Pbl}_{3}$ (MAPI) calculated using HSEO6 with the addition of spin-orbit coupling. $(a, c)$ Projected density of states and $(b, d)$ band structure for MAPSI and MAPI, respectively. The VBM is set to $0 \mathrm{eV}$ in all cases. The conduction and valence bands of (b) and (d) are denoted by orange and blue lines, respectively.
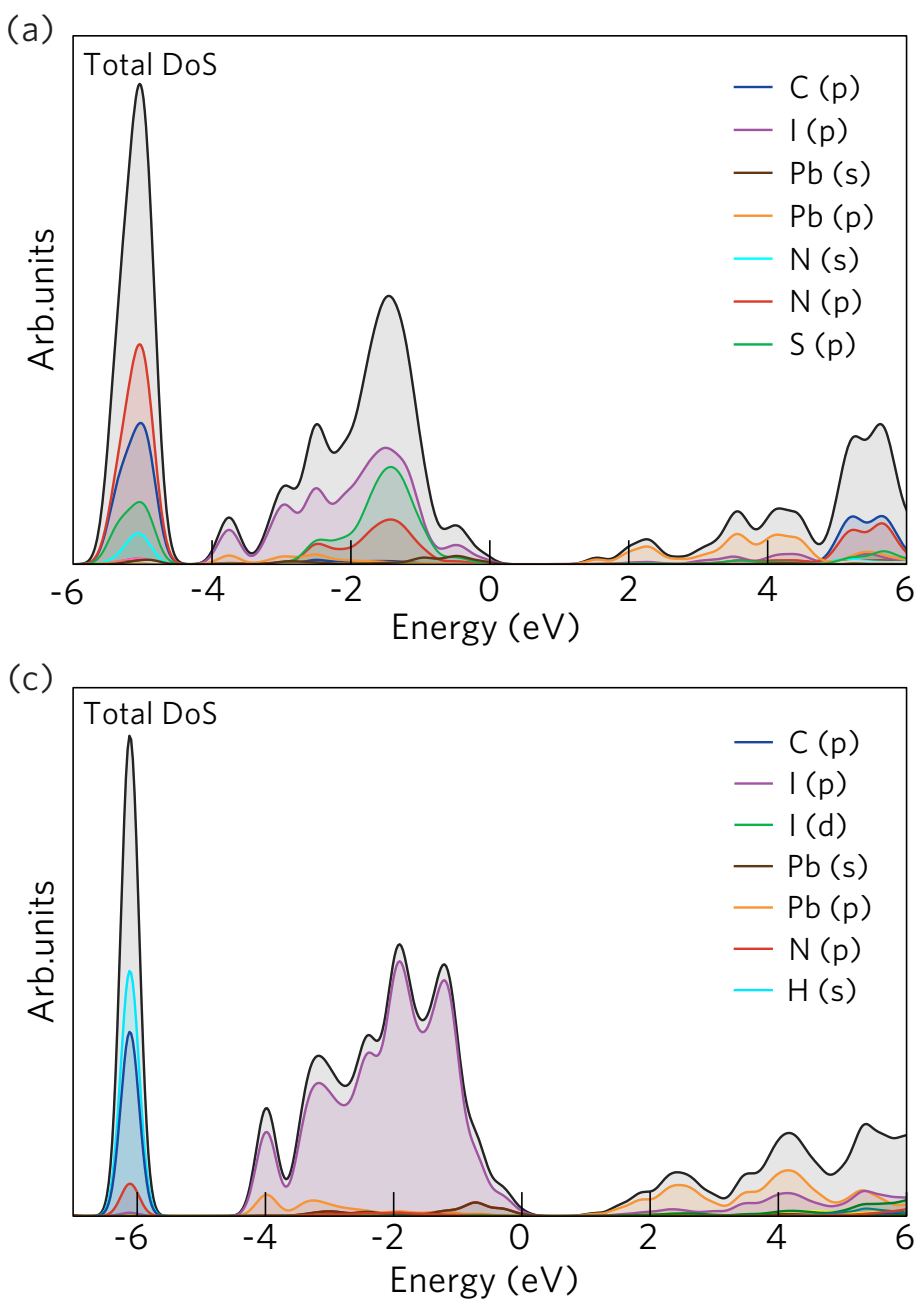
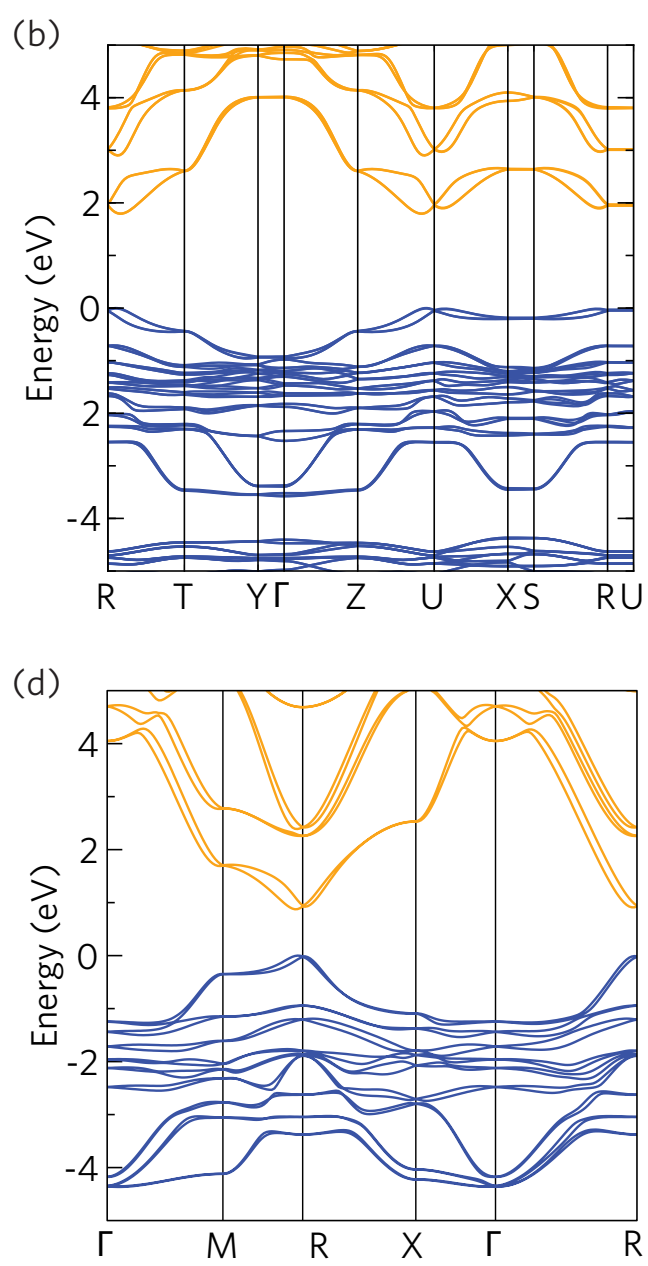


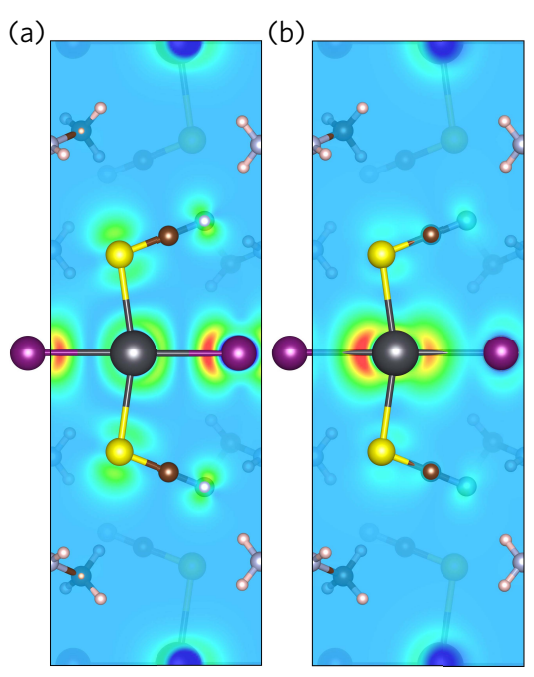

FIGURE 5.4: Charge density isosurfaces of the (a) VBM and (b) $\mathrm{CBM}$ of $\left(\mathrm{CH}_{3} \mathrm{NH}_{3}\right)_{2} \mathrm{~Pb}(\mathrm{SCN})_{2} \mathrm{I}_{2}$, calculated using HSEO6+SOC. Low and high electron density are indicated by blue and red, respectively. $\mathrm{Pb}, \mathrm{S}, \mathrm{N}, \mathrm{C}, \mathrm{I}$, and $\mathrm{H}$ atoms shown by grey, yellow, blue, brown, purple and pink spheres, respectively. tronic structure of MAPI, the conduction band minimum (CBM) is composed almost entirely of $\mathrm{Pb} p$ (Fig. 5.3c). Comparing the electronic structures of MAPSI and MAPI, the main difference is the introduction of $\mathrm{N} p$ and $\mathrm{S} p$ states around $1 \mathrm{eV}$ below the VBM.

In semiconductors, the absolute ionisation potential — and therefore the position of the VBM - has been shown to depend on the amount of $d-p$ repulsion present in the system. ${ }^{218}$ Comparing the $\mathrm{Pb} d$ states of MAPSI and MAPI reveals an average shift in energy of $0.15 \mathrm{eV}$. This slight increase in $p-d$ repulsion is insufficient to account for the smaller band gap of MAPSI compared to other 2D hybrid perovskites. Instead, the presence of the SCN states at the top of the valence band must act to push up the VBM, allowing for a small band gap in spite of its layered nature. Analysis of the $\mathrm{S}-\mathrm{C}-\mathrm{N}$ bond lengths corroborates this picture, revealing an increase in the covalent character of the pseudohalide, as indicated by the lengthening of the $\mathrm{C}-\mathrm{N}(1.17 \AA)$ and shortening of the S - C bonds (1.60 $)$ in MAPSI compared to the ionic AgSCN, where the bond lengths are $1.14 \AA$ and $1.78 \AA$, respectively. ${ }^{219}$ The thiocyanate is therefore actively involved in bonding in the MAPSI structure, as evidenced by the charge density isosurfaces of the CBM and VBM (Fig. 5.4), and in contrast to other polyanion containing MAPI-based systems. ${ }^{220}$ From the isosurfaces, the contributions to the VBM from the $\mathrm{S} 3 p$ and $\mathrm{N} 2 p$ orbitals can be seen clearly, whereas the CBM is dominated by $\mathrm{Pb} 6 p$ states.

The band structure of MAPSI, calculated using HSE06+SOC, is presented in Figure 5.3b. The fundamental band gap is $1.79 \mathrm{eV}$, with the VBM and CBM situated just off the $\mathrm{R}\left(\frac{1}{2}, \frac{1}{2}, \frac{1}{2}\right)$ and $\mathrm{U}\left(0, \frac{1}{2}, \frac{1}{2}\right)$ points, respectively. Very little dispersion is seen across the $\mathrm{X}-\mathrm{S}$ and $\mathrm{R}-\mathrm{U}$ directions, as expected as these paths cross the layers in the [010] direction. Reasonably strong Rashba splitting is seen in the conduction band and, to a lesser extent, in the valence band, due to the lack of inversion symmetry in the MAPSI crystal structure. As previously discussed, such Rashba splitting has been shown to result in dramatically reduced rates of radiative recombination and longer chargecarrier diffusion lengths in MAPI. ${ }^{26}$ 
The effective masses of the CBM and VBM were found to be small $-0.14 m_{0}$ and $0.20 m_{0}$, respectively. These are similar in magnitude to those of MAPI ( $0.15 m_{0}$ and $0.12 m_{0}$, for electrons and holes, respectively ${ }^{221}$ and indicate that both electrons and holes should be mobile in the system. However, we stress that due to the $2 \mathrm{D}$ crystal structure and limited dispersion seen in the [010] direction, conductivity is expected to be strongly anisotropic. A large dielectric constant, $\varepsilon_{\mathrm{r}}$, is increasingly considered a desirable property for a solar absorber due to it's role in screening charged defects and aiding electron-hole separation. The dielectric response of MAPSI was found to be reasonably anisotropic, with values of 16.9, 7.4, and 16.7 calculated for the [100], [010], and [001] directions, respectively. As expected due to the loss in connectivity of the iodide octahedra, these are smaller than those found in the cubic hybrid perovskites, which are often $60-70,{ }^{221}$ but are greater than in other third-generation absorbers such as CZTS ( 9). ${ }^{222}$

Spin-orbit coupling was found to play a significant role in the electronic structure, with a $0.68 \mathrm{eV}$ relativistic lowering of the conduction band observed (depicted in Fig. A.1 of Appendix A). This demonstrates that inclusion of relativistic effects is vital to accurately describe the electronic structure of MAPSI. We note that while manybody effects, such as electron-hole interactions, will also likely play a fundamental role in this system, ${ }^{223,224}$ the size of the MAPSI unit cell (50 atoms) precludes their inclusion in this study.

\section{Stability}

MAPI's chemical stability has been the topic of much debate over the past five years. ${ }^{49,50}$ While it is known that atmospheric moisture content causes rapid degradation of MAPI films, ${ }^{138,225}$ there is growing theoretical and experimental evidence that suggests the intrinsic material is itself thermodynamically unstable with respect to phase separation into $\mathrm{CH}_{3} \mathrm{NH}_{3} \mathrm{I}_{3}$ and $\mathrm{PbI}_{2}$ (the synthetic starting materials). ${ }^{50,139,140}$ To investigate the stability of MAPSI, we have trialled three decomposition pathways, in addition to the decomposi- 
tion route of MAPI as a comparison:

$$
\begin{gathered}
\mathrm{CH}_{3} \mathrm{NH}_{3} \mathrm{PbI}_{3} \rightarrow \mathrm{CH}_{3} \mathrm{NH}_{3} \mathrm{I}+\mathrm{PbI}_{2}, \\
\Delta_{\mathrm{d}} \mathrm{H}=-0.09 \mathrm{eV} \\
\left(\mathrm{CH}_{3} \mathrm{NH}_{3}\right)_{2} \mathrm{~Pb}(\mathrm{SCN})_{2} \mathrm{I}_{2} \rightarrow 2 \mathrm{CH}_{3} \mathrm{NH}_{3} \mathrm{I}+\mathrm{Pb}(\mathrm{SCN})_{2}, \\
\Delta_{\mathrm{d}} \mathrm{H}=0.38 \mathrm{eV} \\
\left(\mathrm{CH}_{3} \mathrm{NH}_{3}\right)_{2} \mathrm{~Pb}(\mathrm{SCN})_{2} \mathrm{I}_{2} \rightarrow 2 \mathrm{CH}_{3} \mathrm{NH}_{3}(\mathrm{SCN})+\mathrm{PbI}_{2}, \\
\Delta_{\mathrm{d}} \mathrm{H}=1.97 \mathrm{eV} \\
\left(\mathrm{CH}_{3} \mathrm{NH}_{3}\right)_{2} \mathrm{~Pb}(\mathrm{SCN})_{2} \mathrm{I}_{2} \rightarrow \mathrm{CH}_{3} \mathrm{NH}_{3} \mathrm{SCN}+\frac{1}{2} \mathrm{CH}_{3} \mathrm{NH}_{3} \mathrm{PbI} \\
\frac{1}{2} \mathrm{CH}_{3} \mathrm{NH}_{3} \mathrm{I}+\frac{1}{2} \mathrm{~Pb}(\mathrm{SCN})_{2}, \\
\Delta_{\mathrm{d}} H=1.20 \mathrm{eV}
\end{gathered}
$$

Unlike in MAPI, where the negative enthalpy of decomposition $\left(\Delta_{\mathrm{d}} H\right)$ indicates it is energetically favourable to spontaneously decompose into $\mathrm{CH}_{3} \mathrm{NH}_{3} \mathrm{I}_{3}$ and $\mathrm{PbI}_{2}$, the decomposition routes for MAPSI are all positive, revealing that phase separation is unfavourable. These results suggest a likely source of the increase in stability reported for hybrid halide materials with SCN incorporation.

\section{Intrinsic defects}

The fundamental defect chemistry of a material will determine its ability to show intrinsic $n$ - or $p$-type conductivity. Accordingly, understanding the defect behaviour of photovoltaics is instrumental in assessing their real-world performance. To this end, we have investigated a range of intrinsic donor and acceptor vacancy defects in MAPSI, namely: two iodine vacancies (due to the presence of two symmetrically inequivalent iodine sites in the MAPSI structure), $V_{\mathrm{I}}^{1}$ and $V_{\mathrm{I}}^{2}$; a lead vacancy, $V_{\mathrm{Pb}}$; a methylammonium vacancy, $V_{\mathrm{MA}}$; and an SCN vacancy, $V_{\mathrm{SCN}}$. The PBEsol calculated charge-state transition level diagram is presented in Figure 5.5.

Of the two $V_{I}$ defects, one has both transition levels resonant in the conduction band, the other possesses a resonant $-1 / 0$ level and a 


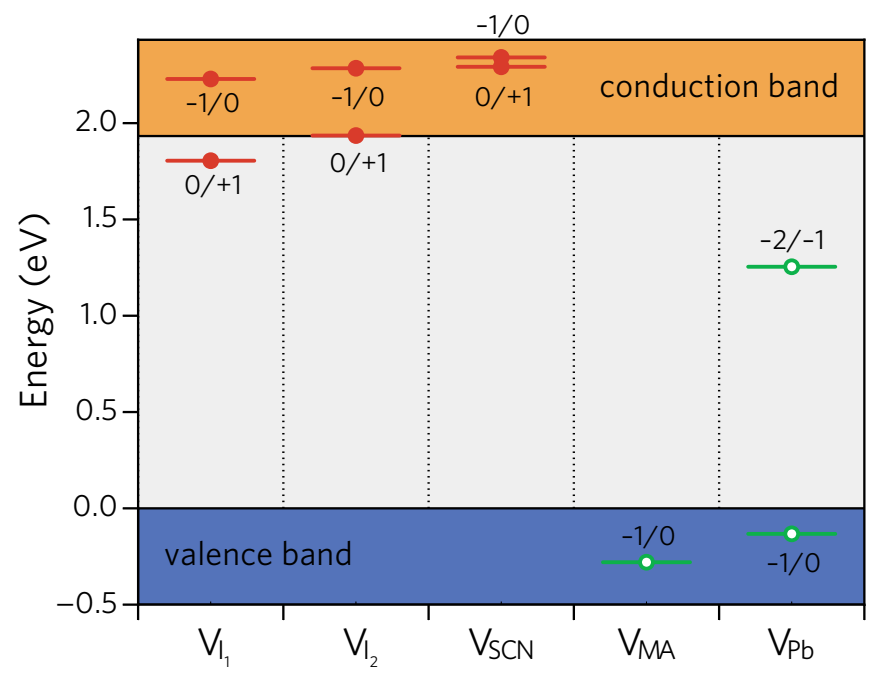

reasonably shallow $0 /+1$ transition state $0.15 \mathrm{eV}$ below the CBM. Additionally, the $V_{\mathrm{SCN}}$ donor defect possesses one $\%+1$ transition level resonant in the conduction band. Considering the acceptor defects: the methylammonium vacancy, $V_{\mathrm{MA}}$, is resonant in valence band, as expected due to the limited bonding interaction between the organic and inorganic components. In contrast, $V_{\mathrm{Pb}}$ possesses a shallow $-1 / 0$ transition level $0.11 \mathrm{eV}$ above the valence band maximum but an ultra-deep $-2 /-1$ level $0.68 \mathrm{eV}$ below the conduction band edge, which may play a role in charge-carrier recombination. While the observation of shallow or resonant transition levels for the $V_{\mathrm{MA}}, V_{\mathrm{SCN}}$, and $V_{\mathrm{I}}$ defects is consistent with previous reports in the literature, our prediction of an ultradeep $V_{\mathrm{Pb}}$ is at odds to the work of Xiao et al. who reported a shallower $-2 /-1$ level $0.44 \mathrm{eV}$ above the VBM. ${ }^{226}$ The cause of this discrepancy may be due to the different computational parameters used in our calculations, namely, we have employed a larger supercell (450 versus 400 atoms), tighter convergence criteria during geometry relaxations $\left(0.01 \mathrm{eV}^{-1}\right.$ versus $\left.0.05 \mathrm{eV}^{-1}\right)$ and our use of the PBEsol exchange-correlation functional versus $\mathrm{PBE}$.

\section{MAPSI structured analogues}

The confirmation of MAPSI as a suitable absorber material for photovoltaic top cells opens up some fundamental questions. Similarly to the $\mathrm{ABX}_{3}$ structured perovskites, which can be tuned on the $\mathrm{A}$,
FIGURE 5.5: Charge-state transition level diagram for a range of intrinsic vacancy defects in $\left(\mathrm{CH}_{3} \mathrm{NH}_{3}\right)_{2} \mathrm{~Pb}(\mathrm{SCN})_{2} \mathrm{I}_{2}$, calculated using PBEsol. Red bands with filled circles denote donor defects, green bands with open circles denote acceptor defects. 
$\mathrm{B}$ and $\mathrm{X}$ sites, can MAPSI also function as a parent compound to a range of analogues possessing the same structural motif? Chemically, it should be viable to replace $\mathrm{Pb}$ with other $2+$ cations such as $\mathrm{Sn}$, I with $\mathrm{Cl}$ and $\mathrm{Br}$, and $\mathrm{SCN}$ with other pseudohalides such as OCN and $\mathrm{SeCN}$. We have therefore considered a total of 17 analogue compounds, comprising all possible compositions of $\left(\mathrm{CH}_{3} \mathrm{NH}_{3}\right)_{2} \mathrm{MPs}_{2} \mathrm{X}_{2}$ (where $\mathrm{M}=\mathrm{Sn}, \mathrm{Pb}$; Ps = OCN, SCN, SeCN; and $\mathrm{X}=\mathrm{Cl}, \mathrm{Br}, \mathrm{I}$ ). As all replacement elements are isoelectronic with their counterparts, the geometric and electronic structure of MAPSI should remain relatively unperturbed. Indeed, when structural relaxations were performed using the PBEsol functional, all compounds retained the orthorhombic $P n m 2_{1}$ space group of MAPSI, with only subtle distortions of the local bonding observed.

\section{Stabilities}

In order to check the stability of the analogues, we have computed the enthalpy of decomposition using PBEsol, for three routes comparable to those tested for MAPSI. For $\left(\mathrm{CH}_{3} \mathrm{NH}_{3}\right)_{2} \mathrm{MPs}_{2} \mathrm{X}_{2}$ (where $\mathrm{M}=\mathrm{Sn}$, $\mathrm{Pb}$; $\mathrm{Ps}=\mathrm{OCN}, \mathrm{SCN}, \mathrm{SeCN}$; and $\mathrm{X}=\mathrm{Cl}, \mathrm{Br}, \mathrm{I})$ the decomposition paths investigated were:

$$
\begin{aligned}
\left(\mathrm{CH}_{3} \mathrm{NH}_{3}\right)_{2} \mathrm{MPs}_{2} \mathrm{X}_{2} & \rightarrow 2 \mathrm{CH}_{3} \mathrm{NH}_{3} \mathrm{X}+\mathrm{MPs}_{2} \\
\left(\mathrm{CH}_{3} \mathrm{NH}_{3}\right)_{2} \mathrm{MPs}_{2} \mathrm{X}_{2} \rightarrow & 2 \mathrm{CH}_{3} \mathrm{NH}_{3} \mathrm{Ps}+\mathrm{MX}_{2} \\
\left(\mathrm{CH}_{3} \mathrm{NH}_{3}\right)_{2} \mathrm{MPs}_{2} \mathrm{X}_{2} \rightarrow & \mathrm{CH}_{3} \mathrm{NH}_{3} \mathrm{Ps}+\frac{1}{2} \mathrm{CH}_{3} \mathrm{NH}_{3} \mathrm{MX}_{3} \\
& +\frac{1}{2} \mathrm{CH}_{3} \mathrm{NH}_{3} \mathrm{X}+\frac{1}{2} \mathrm{MPs}_{2}
\end{aligned}
$$

The enthalpies of decomposition, $\Delta_{\mathrm{d}} H$, for all compounds and decomposition routes are shown in Table 5.2. The enthalpies of decomposition were positive across the board, suggesting that all analogues should be stable against phase separation. 


\begin{tabular}{|c|c|c|c|}
\hline \multirow[b]{2}{*}{ Compound } & \multicolumn{3}{|c|}{$\Delta_{\mathrm{d}} H(\mathrm{eV})$} \\
\hline & Eqn. 5.5 & Eqn. 5.6 & Eqn. 5.7 \\
\hline $\mathrm{MA}_{2} \mathrm{~Pb}(\mathrm{OCN})_{2} \mathrm{Cl}_{2}$ & 0.05 & 2.66 & 0.72 \\
\hline $\mathrm{MA}_{2} \mathrm{~Pb}(\mathrm{SCN})_{2} \mathrm{Cl}_{2}$ & 0.08 & 2.99 & 0.90 \\
\hline $\mathrm{MA}_{2} \mathrm{~Pb}(\mathrm{SeCN})_{2} \mathrm{Cl}_{2}$ & 0.11 & 3.23 & 1.03 \\
\hline $\mathrm{MA}_{2} \mathrm{~Pb}(\mathrm{OCN})_{2} \mathrm{Br}_{2}$ & 0.10 & 0.63 & 0.85 \\
\hline $\mathrm{MA}_{2} \mathrm{~Pb}(\mathrm{SeCN})_{2} \mathrm{Br}_{2}$ & 0.25 & 1.29 & 1.25 \\
\hline $\mathrm{MA}_{2} \mathrm{~Pb}(\mathrm{OCN})_{2} \mathrm{I}_{2}$ & 0.13 & 1.41 & 0.79 \\
\hline $\mathrm{MA}_{2} \mathrm{~Pb}(\mathrm{SCN})_{2} \mathrm{I}_{2}$ & 0.38 & 1.97 & 1.20 \\
\hline $\mathrm{MA}_{2} \mathrm{~Pb}(\mathrm{SeCN})_{2} \mathrm{I}_{2}$ & 0.41 & 2.21 & 1.33 \\
\hline $\mathrm{MA}_{2} \mathrm{Sn}(\mathrm{OCN})_{2} \mathrm{Cl}_{2}$ & 4.09 & 1.62 & 2.78 \\
\hline $\mathrm{MA}_{2} \mathrm{Sn}(\mathrm{SCN})_{2} \mathrm{Cl}_{2}$ & 0.16 & 2.09 & 1.04 \\
\hline $\mathrm{MA}_{2} \mathrm{Sn}(\mathrm{SeCN})_{2} \mathrm{Cl}_{2}$ & 0.11 & 2.31 & 1.13 \\
\hline $\mathrm{MA}_{2} \mathrm{Sn}(\mathrm{OCN})_{2} \mathrm{Br}_{2}$ & 4.19 & 1.66 & 2.87 \\
\hline $\mathrm{MA}_{2} \mathrm{Sn}(\mathrm{SCN})_{2} \mathrm{Br}_{2}$ & 0.22 & 2.09 & 1.10 \\
\hline $\mathrm{MA}_{2} \mathrm{Sn}(\mathrm{SeCN})_{2} \mathrm{Br}_{2}$ & 0.18 & 2.31 & 2.31 \\
\hline $\mathrm{MA}_{2} \mathrm{Sn}(\mathrm{OCN})_{2} \mathrm{I}_{2}$ & 4.19 & 1.46 & 2.85 \\
\hline $\mathrm{MA}_{2} \mathrm{Sn}(\mathrm{SCN})_{2} \mathrm{I}_{2}$ & 0.34 & 2.01 & 1.20 \\
\hline $\mathrm{MA}_{2} \mathrm{Sn}(\mathrm{SeCN})_{2} \mathrm{I}_{2}$ & 0.32 & 2.25 & 1.31 \\
\hline
\end{tabular}

\section{Electronic properties}

The fundamental direct and indirect band gaps for all analogues are provided in Table 5.3. We note that, as in MAPSI, the band gaps are likely to be underestimated relative to the room temperature optical band gaps. In contrast to MAPSI, where the fundamental band gap is direct, most of the analogues posses slightly indirect gaps. The difference between direct and indirect transitions is, however, small (between 10-40 meV), and will therefore have a limited effect on the optical absorption.

The size of the band gaps is controlled by the $\mathrm{X}$ site anion: when moving down group 17, the band gap is reduced from $\sim 3.0 \mathrm{eV}(\mathrm{Cl})$ to $\sim 2.4 \mathrm{eV}(\mathrm{Br})$ and $\sim 1.8 \mathrm{eV}(\mathrm{I})$. An examination of the density of states of MAPSI can rationalise this behaviour: the upper valence band is composed almost entirely of halide $p$ states, with the conduction band dominated by $\mathrm{Pb} p$ states. Accordingly, the valence band character causes the band gap reduction seen down the series, in line with the binding energy of the halide $p$ orbitals. In comparison, the pseu-
TABLE 5.2: Enthalpy of decomposition, $\Delta_{\mathrm{d}} H$, with respect to Equations 5.5, 5.6, and 5.7, for $\left(\mathrm{CH}_{3} \mathrm{NH}_{3}\right)_{2} \mathrm{MPs}_{2} \mathrm{X}_{2}$, where $\mathrm{M}=$ $\mathrm{Sn}, \mathrm{Pb} ; \mathrm{Ps}=\mathrm{OCN}, \mathrm{SCN}, \mathrm{SeCN}$; and $\mathrm{X}=\mathrm{Cl}, \mathrm{Br}, \mathrm{l}$, calculated using PBEsol 
TABLE 5.3: Electronic properties of $\left(\mathrm{CH}_{3} \mathrm{NH}_{3}\right)_{2} \mathrm{MPs}_{2} \mathrm{X}_{2}$, where $\mathrm{M}$ $=\mathrm{Sn}, \mathrm{Pb} ; \mathrm{Ps}=\mathrm{OCN}, \mathrm{SCN}, \mathrm{SeCN}$; and $\mathrm{X}=\mathrm{Cl}, \mathrm{Br}, \mathrm{l}$, calculated using HSEO6+SOC. Indirect and direct band gaps denoted by $E_{\mathrm{g}}^{\text {ind }}$ and $E_{\mathrm{g}}^{\mathrm{dir}}$, respectively. Hole and electron effective masses are given by $m_{\mathrm{h}}^{*}$ and $m_{\mathrm{e}}^{*}$. IP and EA stand for ionisation potential and electron affinity, respectively. $\perp$ and $\|$ indicate properties perpendicular and parallel to the two-dimensional perovskite sheets, respectively

\begin{tabular}{|c|c|c|c|c|c|c|c|}
\hline Compound & $\begin{array}{l}\mathrm{E}_{\mathrm{g}}^{\text {ind }} \\
(\mathrm{eV})\end{array}$ & $\begin{array}{c}\mathrm{E}_{\mathrm{g}}^{\mathrm{dir}} \\
(\mathrm{eV})\end{array}$ & $\begin{array}{l}m_{h}^{* \perp} \\
\left(m_{0}\right)\end{array}$ & $\begin{array}{l}m_{\mathrm{h}}^{* \|} \\
\left(m_{0}\right)\end{array}$ & $\begin{array}{l}m_{\mathrm{e}}^{* \|} \\
\left(m_{0}\right)\end{array}$ & $\begin{array}{c}\mathrm{IP} \\
(\mathrm{eV})\end{array}$ & $\begin{array}{c}\text { EA } \\
(\mathrm{eV})\end{array}$ \\
\hline $\mathrm{MA}_{2} \mathrm{~Pb}(\mathrm{OCN})_{2} \mathrm{Cl}_{2}$ & 3.47 & 3.51 & 41.10 & 0.59 & 0.42 & 7.14 & 3.67 \\
\hline $\mathrm{MA}_{2} \mathrm{~Pb}(\mathrm{SCN})_{2} \mathrm{Cl}_{2}$ & 3.03 & 3.05 & 40.00 & 3.07 & 0.50 & 6.50 & 3.94 \\
\hline $\mathrm{MA}_{2} \mathrm{~Pb}(\mathrm{SeCN})_{2} \mathrm{Cl}_{2}$ & 3.04 & 3.05 & 38.89 & 14.09 & 0.59 & 5.68 & 3.93 \\
\hline $\mathrm{MA}_{2} \mathrm{~Pb}(\mathrm{OCN})_{2} \mathrm{Br}_{2}$ & 2.56 & 2.58 & - & 0.34 & 0.28 & 6.38 & 3.34 \\
\hline $\mathrm{MA}_{2} \mathrm{~Pb}(\mathrm{SCN})_{2} \mathrm{Br}_{2}$ & 2.30 & 2.32 & 36.17 & 0.44 & 0.25 & 6.03 & 3.73 \\
\hline $\mathrm{MA}_{2} \mathrm{~Pb}(\mathrm{SeCN})_{2} \mathrm{Br}_{2}$ & 2.31 & 2.33 & - & 1.89 & 0.29 & 5.55 & 3.76 \\
\hline $\mathrm{MA}_{2} \mathrm{~Pb}(\mathrm{OCN})_{2} \mathrm{I}_{2}$ & 1.75 & 1.76 & - & 0.22 & 0.18 & 6.27 & 3.23 \\
\hline $\mathrm{MA}_{2} \mathrm{~Pb}(\mathrm{SCN})_{2} \mathrm{I}_{2}$ & 1.79 & 1.79 & - & 0.31 & 0.20 & 5.91 & 3.60 \\
\hline $\mathrm{MA}_{2} \mathrm{~Pb}(\mathrm{SeCN})_{2} \mathrm{I}_{2}$ & 1.82 & 1.85 & - & 0.86 & 0.19 & 5.51 & 3.68 \\
\hline $\mathrm{MA}_{2} \mathrm{Sn}(\mathrm{OCN})_{2} \mathrm{Cl}_{2}$ & 4.03 & 4.05 & 39.52 & 1.00 & 3 & 7 & 2.64 \\
\hline $\mathrm{MA}_{2} \mathrm{Sn}(\mathrm{SCN})_{2} \mathrm{Cl}_{2}$ & 3.74 & 3.74 & 38.74 & 20.42 & 0.96 & 5.94 & 2.88 \\
\hline $\mathrm{MA}_{2} \mathrm{Sn}(\mathrm{SeCN})_{2} \mathrm{Cl}_{2}$ & 3.56 & 3.57 & 37.59 & 38.66 & 0.86 & 5.13 & 3.22 \\
\hline $\mathrm{MA}_{2} \mathrm{Sn}(\mathrm{OCN})_{2} \mathrm{Br}_{2}$ & 3.06 & 3.08 & - & 0.36 & 0.43 & 6.06 & 2.32 \\
\hline $\mathrm{MA}_{2} \mathrm{Sn}(\mathrm{SCN})_{2} \mathrm{Br}_{2}$ & 2.98 & 2.98 & - & 2.15 & 0.63 & 5.67 & 2.69 \\
\hline $\mathrm{MA}_{2} \mathrm{Sn}(\mathrm{SeCN})_{2} \mathrm{Br}_{2}$ & 2.96 & 2.96 & - & 3.85 & 0.64 & 4.79 & 3.12 \\
\hline $\mathrm{MA}_{2} \mathrm{Sn}(\mathrm{OCN})_{2} \mathrm{I}_{2}$ & 1.90 & 1.92 & - & 0.19 & 0.32 & 5.90 & 2.33 \\
\hline $\mathrm{MA}_{2} \mathrm{Sn}(\mathrm{SCN})_{2} \mathrm{I}_{2}$ & 1.67 & 1.69 & - & 0.51 & 0.32 & 5.64 & 2.67 \\
\hline $\mathrm{MA}_{2} \mathrm{Sn}(\mathrm{SeCN})_{2} \mathrm{I}_{2}$ & 1.89 & 1.89 & - & 0.84 & 0.38 & 4.94 & 3.06 \\
\hline
\end{tabular}

dohalide has a much smaller effect on the band gap, with the thiocyanate and selenocyanate showing only slightly smaller band gaps than their cyanate counterparts.

The trend in band gaps between metals is more pronounced, with the tin analogues possessing band gaps an average of $0.40 \mathrm{eV}$ larger than the corresponding lead materials. This behaviour reflects the strength of spin-orbit coupling on the lead valence electrons, which acts to increase the binding energy of the $\mathrm{Pb} 5 p$ orbitals and lower the conduction band minimum. The aforementioned trends of the pseudohalides and metals are not seen in the band gaps of the iodides, which show little correlation to composition. In general, the iodides all possess near-direct band gaps in the ideal range for photovoltaic top cells (1.69-1.92 eV), even when considering the HSE06+SOC results are likely underestimated compared to the experimental gaps.

Spin-orbit coupling was found to have a dramatic effect on the band gaps of all materials (full results provided in Table A.1 of Appendix A). The reduction in the band gaps of the lead analogues ( 0.6$0.9 \mathrm{eV})$ was significantly larger than in their tin counterparts $(\sim 0.1-$ $0.2 \mathrm{eV}$ ), as spin-orbit coupling interactions scale approximately as 

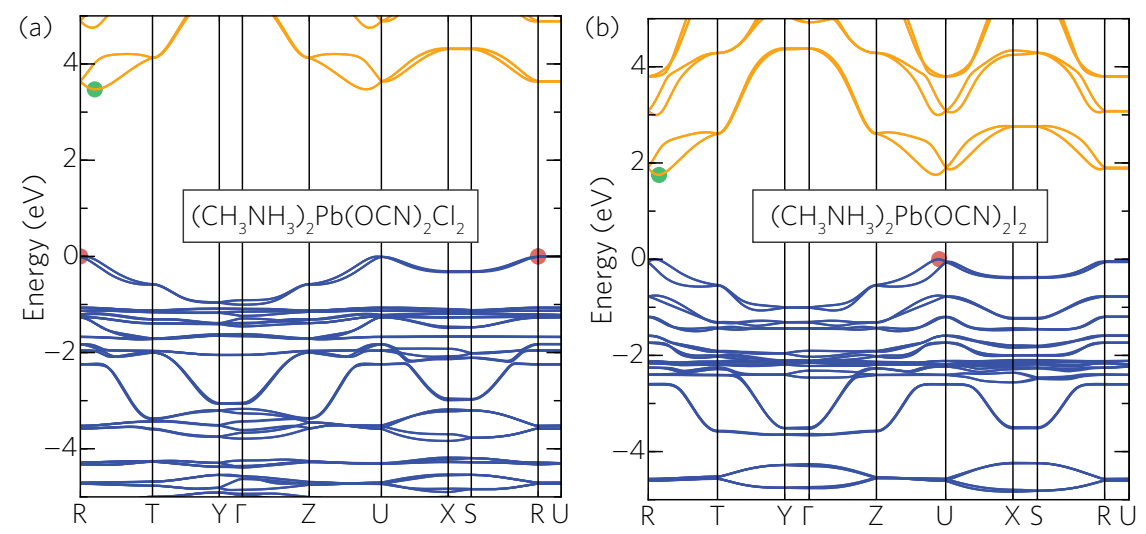

FIGURE 5.6: HSEO6+SOC calculated band structure of MAPSIstructured cyanate compounds: a) $\left(\mathrm{CH}_{3} \mathrm{NH}_{3}\right)_{2} \mathrm{~Pb}(\mathrm{OCN})_{2} \mathrm{Cl}_{2}$, b) $\left(\mathrm{CH}_{3} \mathrm{NH}_{3}\right)_{2} \mathrm{~Pb}(\mathrm{OCN})_{2} \mathrm{I}_{2}, \quad$ c) $\left(\mathrm{CH}_{3} \mathrm{NH}_{3}\right)_{2} \mathrm{Sn}(\mathrm{OCN})_{2} \mathrm{Cl}_{2}$, and d) $\left(\mathrm{CH}_{3} \mathrm{NH}_{3}\right)_{2} \mathrm{Sn}(\mathrm{OCN})_{2} \mathrm{I}_{2}$. The valence band maximum is set to $\mathrm{O} \mathrm{eV}$. Valence and conduction bands indicated by blue and orange lines, respectively.
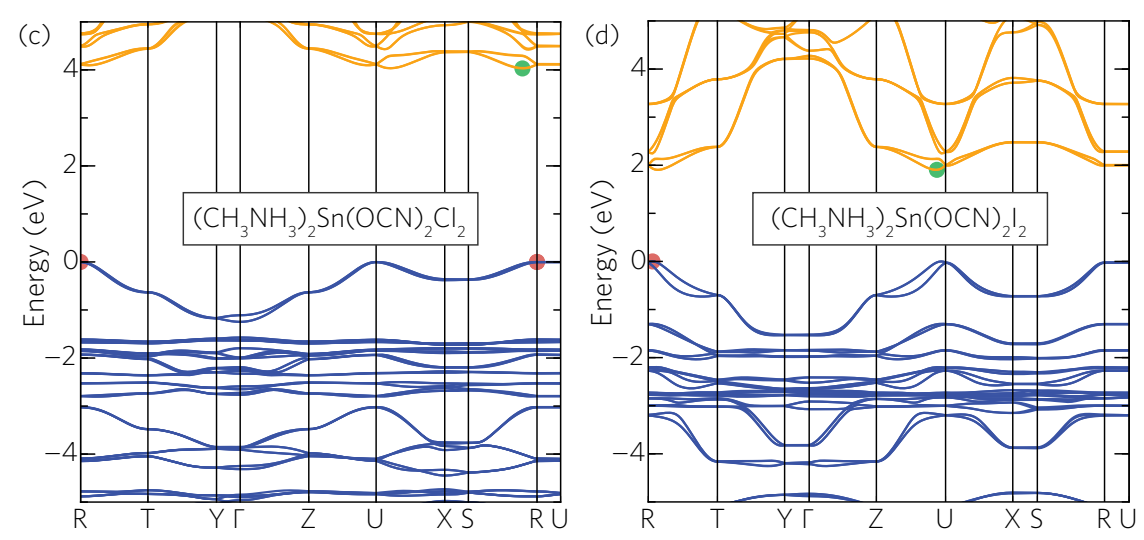

$\mathrm{Z}^{4}$, where $\mathrm{Z}$ is the atomic mass. These results stress the need to properly account for relativistic effects when modelling lead and tin hybrid perovskite-type materials.

To illustrate the effect of the halide on electronic properties, a selection of band structures for the tin and lead cyanate analogues are provided in Figure 5.6 (the full set of band structures for each composition can be found in Appendix A). Moving down the halides, the reduction in band gap is apparent, with the width of the valence and conduction bands remaining roughly constant for all compositions. In both the lead and tin chlorides, the valence band maximum is found at the $\mathrm{R}$ point $\left(\frac{\overline{1}}{2}, \frac{1}{2}, \frac{1}{2}\right)$ and shifts slightly to the side of either the $\mathrm{R}$ or $\mathrm{U}\left(0, \frac{1}{2}, \frac{1}{2}\right)$ points for the bromides, due to greater Rashba splitting in the upper valence bands. The conduction band minimum position does not appear to follow any trends in composition and is found close to the $\mathrm{R}$ or $\mathrm{U}$ points, depending on the asymmetry in Rashba splitting. The reduction in band gap occurs with a concomitant reduction in the band edge effective masses. Con- 
FIGURE 5.7: HSEO6+SOC calculated band structure of MAPSIstructured bromide compounds: a) $\left(\mathrm{CH}_{3} \mathrm{NH}_{3}\right)_{2} \mathrm{Sn}(\mathrm{OCN})_{2} \mathrm{Br}_{2}$, b) $\left(\mathrm{CH}_{3} \mathrm{NH}_{3}\right)_{2} \mathrm{Sn}(\mathrm{SeCN})_{2} \mathrm{Br}_{2}$, $\left(\mathrm{CH}_{3} \mathrm{NH}_{3}\right)_{2} \mathrm{Sn}(\mathrm{OCN})_{2} \mathrm{Br}_{2}$, and d) $\left(\mathrm{CH}_{3} \mathrm{NH}_{3}\right)_{2} \mathrm{Sn}(\mathrm{SeCN})_{2} \mathrm{Br}_{2}$. The valence band maximum is set to $\mathrm{O} \mathrm{eV}$. Valence and conduction bands indicated by blue and orange lines, respectively.
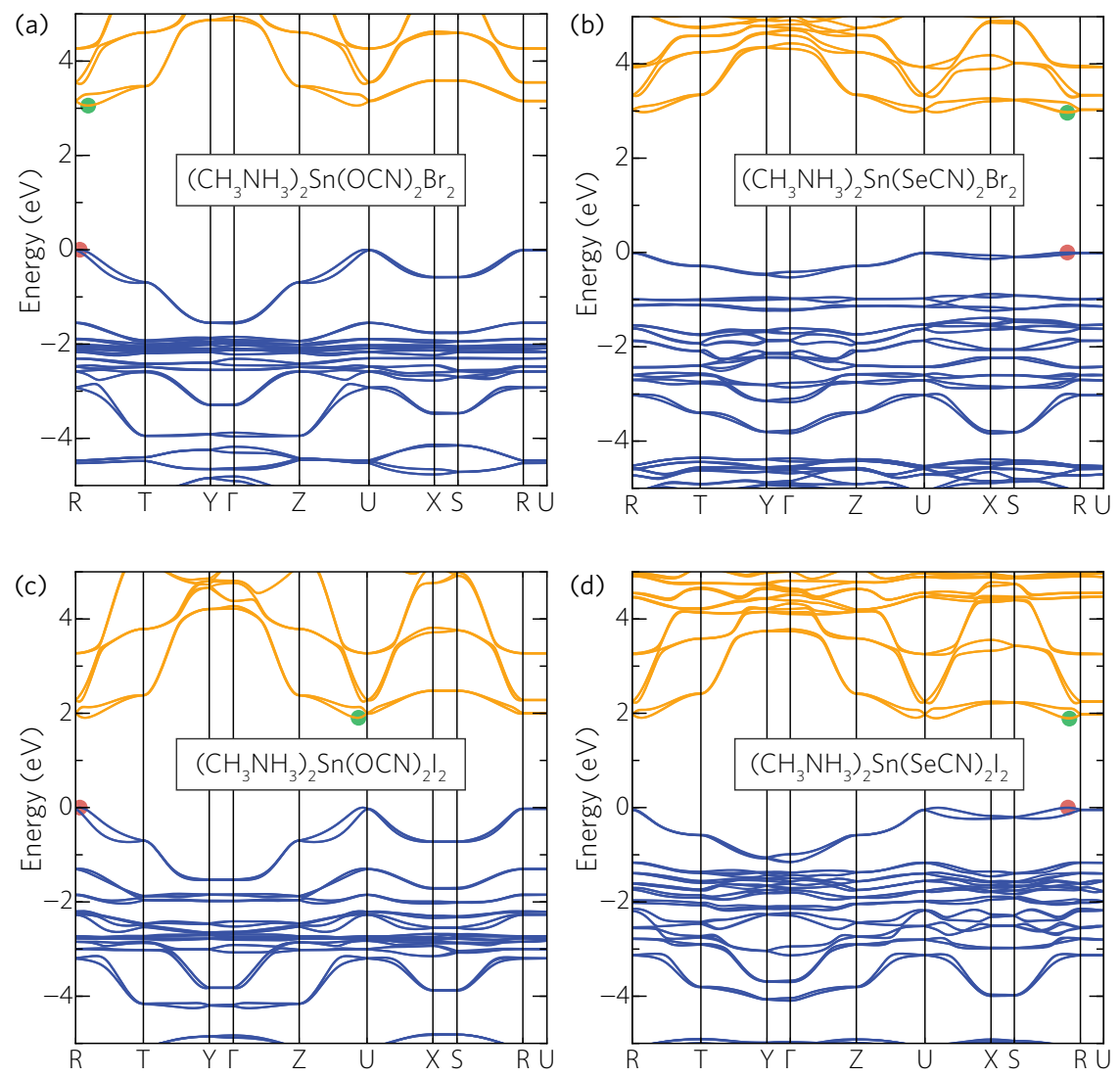

sidering only the two directions parallel to the $2 \mathrm{D}$ perovskite sheets (along [100] and [001], indicated by $m^{* \|}$ ), the hole and electron effective masses are $m_{\mathrm{h}}^{* \|}=0.59 m_{0}, 0.34 m_{0}$, and $0.22 m_{0}$ and $m_{\mathrm{e}}^{* \|}=$ $0.42 m_{0}, 0.28 m_{0}, 0.18 m_{0}$ for $\mathrm{Cl}, \mathrm{Br}$, and I lead cyanates, respectively. In contrast, the effective masses for the directions perpendicular to the inorganic perovskite layers (along [010]) are considerably larger $\left(\sim 40 m_{0}\right)$, as expected due to the 2D structure, which results in limited interactions across the layers.

To illustrate the effect of the pseudohalide on electronic properties, a selection of band structures for the tin bromide and iodide analogues are provided in Figure 5.7 (the full set of band structures for each composition can be found in Appendix A). Moving from OCN to $\mathrm{SCN}$ and SeCN, the valence bandwidth is significantly reduced, with the conduction bandwidth showing a similar, albeit lesser, trend. We note that this effect is stronger in the chlorides and bromides than for the iodides. The pseudohalide plays a role on the in-plane ef- 


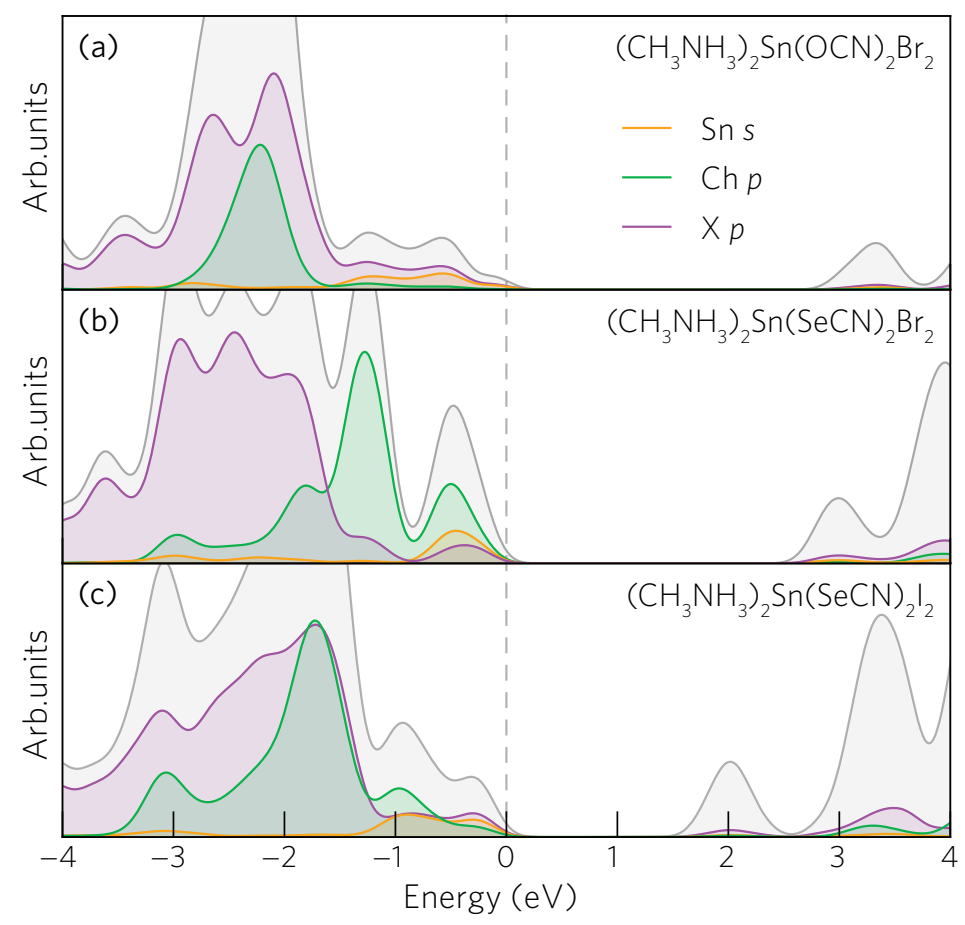

fective masses, with the SCN and SeCN analogues showing masses almost twice as light as those for the OCN compounds. Furthermore, the choice of pseudohalide appears to play a role in controlling the anisotropy in the Rashba splitting of the conduction band minimum. Indeed, while the spin-split pockets appear almost symmetrical in the thiocyanates and selenocyanates, the spin pockets of the cyanates show significant asymmetry. Considering the lower conduction band is dominated by metal $p$ states, this behaviour is likely due to distortion in the local symmetry around the metal site. This is consistent with analysis of the crystal structures, which reveals greater off-centring of the metal cation within the $\mathrm{MX}_{4} \mathrm{Ps}_{2}$ octahedra for the cyanate materials, than in the thiocyanate and selenocyanate analogues. This is likely due to the formation of a stereochemically active lone-pair of electrons in the metal $s^{2}$ orbitals, which, based on the revised lone-pair model, ${ }^{227,228}$ will be most prevalent for cyanate and reduced for the thiocyanate and selenocyanate analogues.

Analysis of these results reveals a trend; while the chlorides and bromides show reduced band gaps and narrower valence bandwidths when moving down the pseudohalides, the iodides do not show the
FIGURE 5.8: Projected density of states for a) $\left(\mathrm{CH}_{3} \mathrm{NH}_{3}\right)_{2} \mathrm{Sn}(\mathrm{OCN})_{2} \mathrm{Br}_{2}$, b) $\left(\mathrm{CH}_{3} \mathrm{NH}_{3}\right)_{2} \mathrm{Sn}(\mathrm{SeCN})_{2} \mathrm{Br}_{2}$, and c) $\quad\left(\mathrm{CH}_{3} \mathrm{NH}_{3}\right)_{2} \mathrm{Sn}(\mathrm{SeCN})_{2} \mathrm{I}_{2}$, highlighting the relative contributions of the chalcogenide $(\mathrm{Ch})$ and halide $(\mathrm{X})$ to the upper valence band. The valence band maximum is set to $0 \mathrm{eV}$ and is indicated by a dashed line. The contributions of the N, C, and Sn $p$ orbitals have been omitted for clarity. 
TABLE 5.4: Dielectric constants $\left(\varepsilon_{\mathrm{r}}\right)$ of $\left(\mathrm{CH}_{3} \mathrm{NH}_{3}\right)_{2} \mathrm{MPs}_{2} \mathrm{X}_{2}$, where $\mathrm{M}=\mathrm{Sn}, \mathrm{Pb} ; \mathrm{Ps}=\mathrm{OCN}$, $\mathrm{SCN}, \mathrm{SeCN}$; and $\mathrm{X}=\mathrm{Cl}, \mathrm{Br}, \mathrm{I}$, calculated using $\mathrm{HSEO6+SOC}$. $\perp$ and $\|$ indicate properties perpendicular and parallel to the $2 \mathrm{D}$ perovskite sheets, respectively

\begin{tabular}{lcc}
\hline Compound & $\boldsymbol{\varepsilon}_{\mathbf{r}}^{\perp}$ & $\varepsilon_{\mathbf{r}}^{\|}$ \\
\hline $\mathrm{MA}_{2} \mathrm{~Pb}(\mathrm{OCN})_{2} \mathrm{Cl}_{2}$ & 7.2 & 11.4 \\
$\mathrm{MA}_{2} \mathrm{~Pb}(\mathrm{SCN})_{2} \mathrm{Cl}_{2}$ & 6.6 & 19.5 \\
$\mathrm{MA}_{2} \mathrm{~Pb}(\mathrm{SeCN})_{2} \mathrm{Cl}_{2}$ & 6.4 & 19.0 \\
$\mathrm{MA}_{2} \mathrm{~Pb}(\mathrm{OCN})_{2} \mathrm{Br}_{2}$ & 8.4 & 9.1 \\
$\mathrm{MA}_{2} \mathrm{~Pb}(\mathrm{SCN})_{2} \mathrm{Br}_{2}$ & 8.3 & 19.6 \\
$\mathrm{MA}_{2} \mathrm{~Pb}(\mathrm{SeCN})_{2} \mathrm{Br}_{2}$ & 7.4 & 16.8 \\
$\mathrm{MA}_{2} \mathrm{~Pb}(\mathrm{OCN})_{2} \mathrm{I}_{2}$ & 9.3 & 8.5 \\
$\mathrm{MA}_{2} \mathrm{~Pb}(\mathrm{SCN})_{2} \mathrm{I}_{2}$ & 9.0 & 21.9 \\
$\mathrm{MA}_{2} \mathrm{~Pb}(\mathrm{SeCN})_{2} \mathrm{I}_{2}$ & 9.2 & 17.7
\end{tabular}

$\mathrm{MA}_{2} \mathrm{Sn}(\mathrm{OCN})_{2} \mathrm{Cl}_{2} \quad 9.3 \quad 6.1$ $\mathrm{MA}_{2} \mathrm{Sn}(\mathrm{SCN})_{2} \mathrm{Cl}_{2} \quad 6.9 \quad 7.0$ $\mathrm{MA}_{2} \mathrm{Sn}(\mathrm{SeCN})_{2} \mathrm{Cl}_{2} \quad 7.0 \quad 11.7$ $\mathrm{MA}_{2} \mathrm{Sn}(\mathrm{OCN})_{2} \mathrm{Br}_{2} \quad 12.5 \quad 7.2$ $\mathrm{MA}_{2} \mathrm{Sn}(\mathrm{SCN})_{2} \mathrm{Br}_{2} \quad 8.3 \quad 8.4$ $\mathrm{MA}_{2} \mathrm{Sn}(\mathrm{SeCN})_{2} \mathrm{Br}_{2} \quad 8.5 \quad 20.2$ $\mathrm{MA}_{2} \mathrm{Sn}(\mathrm{OCN})_{2} \mathrm{I}_{2} \quad 9.3 \quad 6.9$ $\mathrm{MA}_{2} \mathrm{Sn}(\mathrm{SCN})_{2} \mathrm{I}_{2} \quad 8.7 \quad 7.8$ $\mathrm{MA}_{2} \mathrm{Sn}(\mathrm{SeCN})_{2} \mathrm{I}_{2} \quad 8.8 \quad 13.9$ same pattern. Investigation of the relative energies of the pseudohalide and halide $p$ orbitals reveals the origin of this discrepancy. Here we are chiefly concerned with the energies of the chalcogenide $p$ orbitals (rather than the energies of the $\mathrm{N}$ and $\mathrm{C}$ states), due to their direct bonding to the metal site. Considering the OCN analogues: the upper valence band is dominated by halide $p$ and metal $s$ states, with the $\mathrm{O} p$ orbitals present $\sim 1-2 \mathrm{eV}$ deeper into the valence band (Fig. 5.8a). In these materials, the band gaps, valence bandwidths and hole effective masses are solely determined by the halide, resulting in smaller effective masses in the diffuse iodides than the more localised bromides and chlorides. As the S $3 p$ and Se $4 p$ states are higher in energy (10.4 eV and $9.8 \mathrm{eV}$, respectively) ${ }^{229}$ than the chloride and bromide $p$ states $(13.0 \mathrm{eV}$ and $11.8 \mathrm{eV}),{ }^{229}$ the valence band maximum in the thiocyanates and selenocynates shows considerably greater chalcogen contribution. As such, the dispersion at the valence band edge is reduced, due to the greater localisation of these states (Fig. 5.8b). In the iodides, however, the reduced binding energy of the I $5 p$ orbitals $\left.(10.4 \mathrm{eV})^{230}\right)$ enables them to hybridise with the $S$ and Se states, resulting in a consistent band gap regardless in the choice of pseudohalide, with limited effects seen on the valence bandwidth.

\section{Dielectric constants}

The PBEsol calculated dielectric constants are provided in Table 5.4. For most compounds, the dielectric constants parallel to the perovskite sheets are larger and show greater variation $\left(\varepsilon_{\mathrm{r}}^{\|}=6.1-21.9\right)$ than for the directions perpendicular to the sheets $\left(\varepsilon_{\mathrm{r}}^{\perp}=6.4-12.5\right)$. The tin compounds generally show smaller dielectric constants than their lead analogues, due to the reduced polarisability of the $\mathrm{Sn}^{2+}$ cation. Considering just the lead-based materials, the halide plays a clear role on the magnitude of $\varepsilon_{\mathrm{r}}^{\|}$, with the iodides and bromides showing larger dielectric constants ( $\sim 20$ and $\sim 18$, respectively) than the chlorides $(\sim 10)$. These trends are broadly reproduced in the tin analogues, with the iodides again showing the largest in-plane dielectric constants $(\sim 12-20)$, in comparison to much smaller constants for 
the bromides $(\sim 8)$ and chlorides $(\sim 7)$. The relatively large dielectric constants of the iodides are particularly promising for photovoltaic applications, where increased screening of charge will limit the effects of scattering and recombination by charged defects and reduce defect binding energies.

\section{Band alignments}

When designing a photovoltaic device, it is essential to achieve close band alignment of the absorber material with the transparent conducting oxide and hole transporting material, to maximise the achievable open-circuit voltage. The band alignments are also crucial in understanding the dopability of a material - a measure of the ease of $n$ or $p$ type doping. To aid the experimental realisation of high efficiency photovoltaics based on MAPSI and its analogues, we have calculated the band alignments of $\left(\mathrm{CH}_{3} \mathrm{NH}_{3}\right)_{2} \mathrm{MPs}_{2} \mathrm{X}_{2}$, (where $\mathrm{M}=$ $\mathrm{Sn}, \mathrm{Pb}$; Ps = OCN, SCN, SeCN; and $\mathrm{X}=\mathrm{Cl}, \mathrm{Br}, \mathrm{I}$ ) using HSE06 with a correction for the HSE06+SOC band gap (Fig. 5.9).

It is immediately clear that the electron affinity (EA) - the distance from the conduction band minimum to the vacuum level shows significantly less variability than the ionisation potential (IP) - the distance from the valence band maximum to the vacuum level.
FIGURE 5.9: Band alignment of a) $\left(\mathrm{CH}_{3} \mathrm{NH}_{3}\right)_{2} \mathrm{PbPs}_{2} \mathrm{X}_{2}$ and b) $\left(\mathrm{CH}_{3} \mathrm{NH}_{3}\right)_{2} \mathrm{SnPs}_{2} \mathrm{X}_{2}$ (where $\mathrm{Ps}=$ OCN, SCN, SeCN; and $X=C l, B r$, I), relative the vacuum level. Electron affinities and band gaps indicated by dashed and solid lines, respectively.

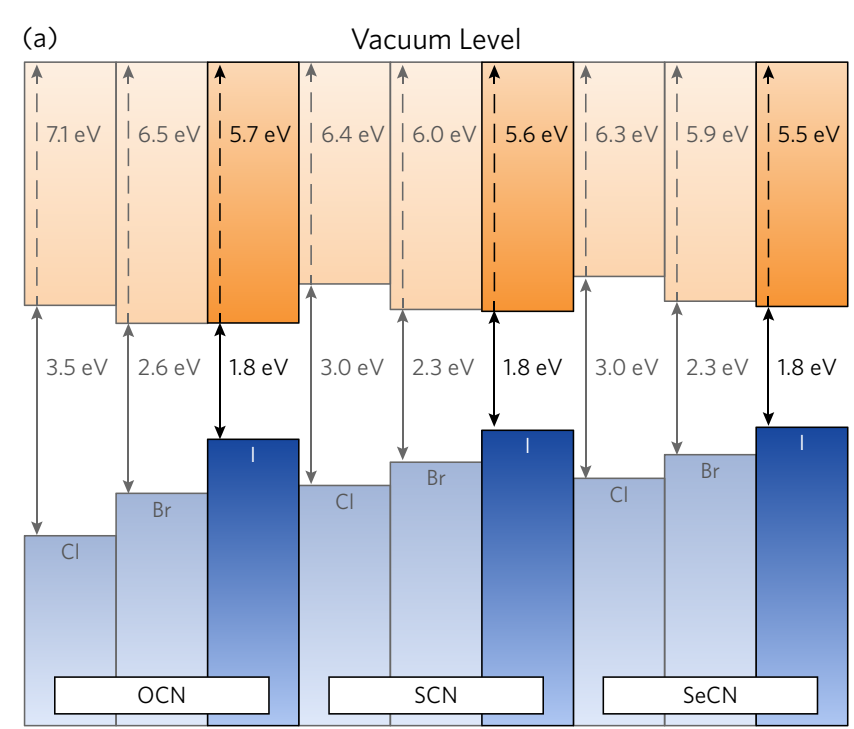

Lead Analogues

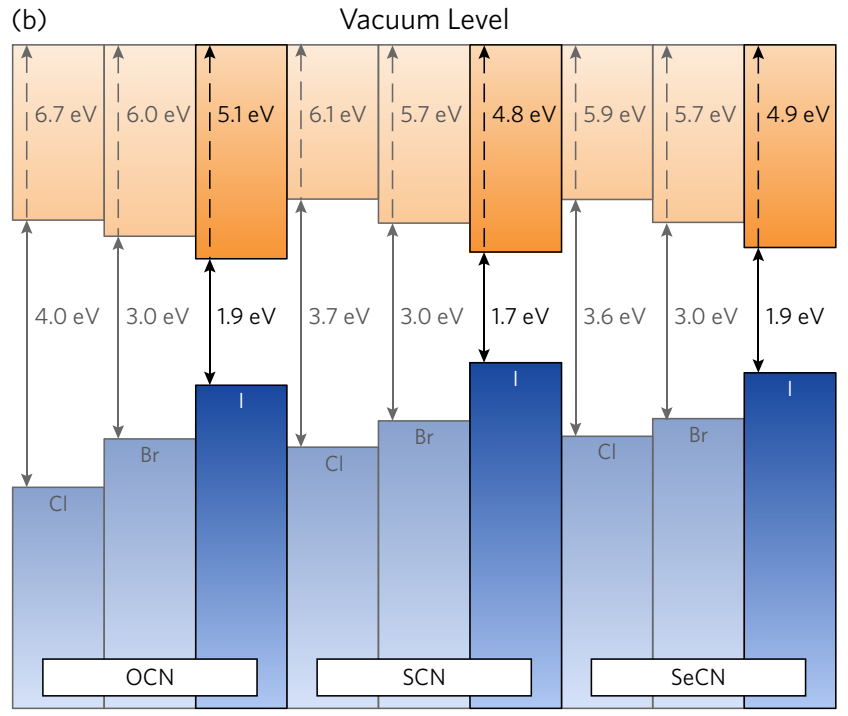

Tin Analogues 
Accordingly, the magnitude of the band gap is controlled through the depth of the valence band maximum, and is therefore determined mainly by the choice in halide. In agreement with the previous densityof-states analysis, the electron affinity and ionisation potential remain relatively unchanged across comparable (i.e. compounds possessing the same halide and metal composition) SCN and SeCN analogues. Overall, the electron affinities and ionisation potentials of the tin compounds are slightly smaller than their lead counterparts, due to the greater relativistic stabilisation effects seen on the $\mathrm{Pb} s$ and $p$ orbitals. The greater electron affinities of the lead analogues is expected to allow for increased ease of $n$-type doping, whereas the larger ionisation potential indicates enhanced stability toward oxidation.

The calculated IP of MAPSI $(5.6 \mathrm{eV})$ is in good agreement with the experimentally determined value $(5.7 \mathrm{eV}) .{ }^{231}$ This is close to the ionisation potential of MAPI $(5.5-5.7 \mathrm{eV}),{ }^{22,35}$ as expected due to the similar valence band composition dominated by $\mathrm{Pb} s$ and I $p$ states. As such, devices based on the lead iodide analogues are expected to form efficient band alignments with spiro-OMeTAD $(\mathrm{IP}=5.2 \mathrm{eV}),{ }^{35}$ a common hole transport layer used in the best performing hybrid halide devices. Additionally, the electron affinity of the lead iodide analogues (3.7-3.9 eV), suggests the absorbers will form favourable alignments with fluorine-doped tin oxide $(\mathrm{FTO}, \mathrm{EA}=4.4 \mathrm{eV}),{ }^{232} \mathrm{a}$ cheap and commercially available transparent conducting oxide.

\section{Defect chemistry}

The defect chemistry of all analogues is broadly similar to that of MAPSI (the transition level diagrams for all compounds are provided in Fig. A.4 of Appendix A). The transition level diagram of $\mathrm{MA}_{2} \mathrm{~Pb}(\mathrm{OCN})_{2} \mathrm{I}_{2}$ is shown in Figure 5.10a. Of the $n$-type defects, the iodide vacancies possess relatively shallow $\%+1$ transition levels $(0.16 \mathrm{eV}$ and $0.08 \mathrm{eV}$ below the conduction band minimum), with the $V_{\mathrm{OCN}}$ defect level resonant in the conduction band. For the $p$ type defects, $V_{\mathrm{MA}}$ is again found to be resonant in the valence band, due to the weak interactions between the methylammonium and per- 

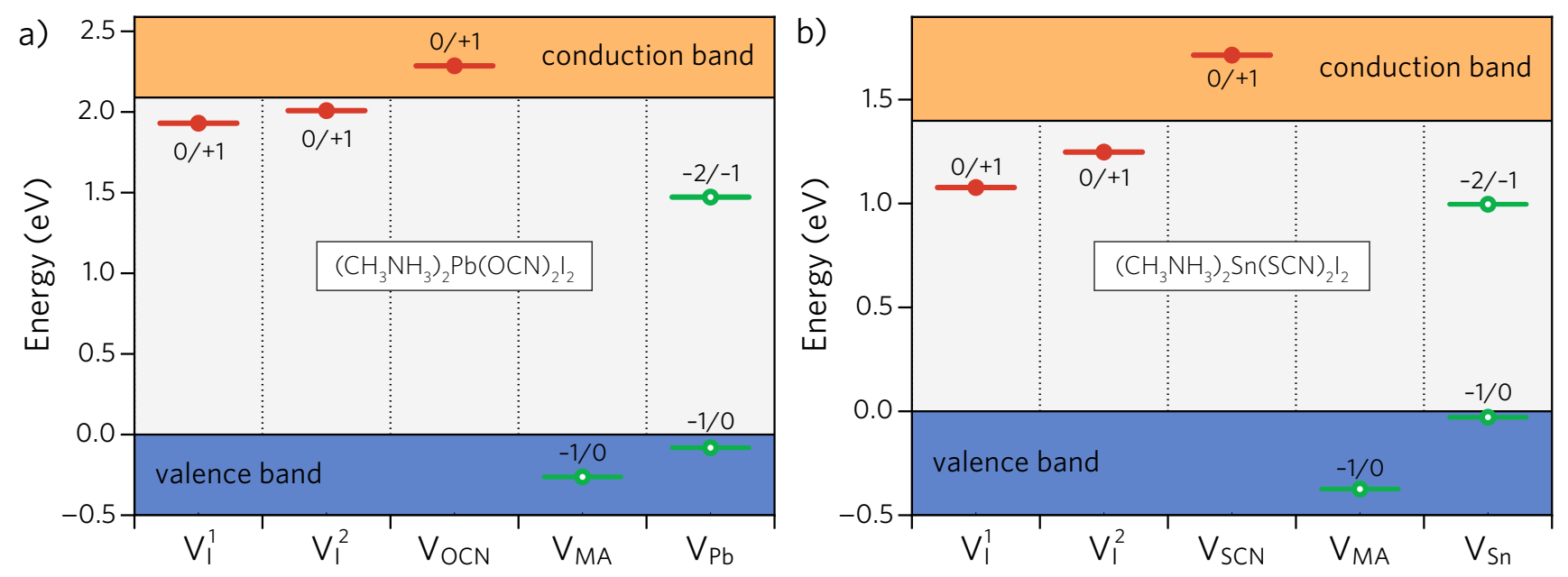

ovskite lattice. Similar to MAPSI, the $V_{\mathrm{Pb}}{ }^{-2} /-1$ defect level is ultradeep in the band gap and therefore may contribute to charge-carrier recombination. Moving from lead to tin, the defect chemistry remains largely unchanged, as indicated by the transition level diagram of $\mathrm{MA}_{2} \mathrm{Sn}(\mathrm{SCN})_{2} \mathrm{I}_{2}$ (Fig. 5.10b). The main difference is found in the iodide vacancies, which possess defect states that are deeper into the gap ( $0.15 \mathrm{eV}$ and $0.32 \mathrm{eV}$ below the conduction band minimum). This can be rationalised by the band alignments, which show the tin analogues possess smaller electron affinities than their lead counterparts. FIGURE 5.10: Calculated charge-state transition level diagrams for a range of intrinsic vacancy defects in a) $\left(\mathrm{CH}_{3} \mathrm{NH}_{3}\right)_{2} \mathrm{~Pb}(\mathrm{OCN})_{2} \mathrm{I}_{2}$, and b) $\left(\mathrm{CH}_{3} \mathrm{NH}_{3}\right)_{2} \mathrm{Sn}(\mathrm{SCN})_{2} \mathrm{I}_{2}$ calculated at the PBEsol level. Red bands with filled circles indicate donor defects, green bands with open circles indicate acceptor defects.

As such, it is likely the iodide vacancy transition levels remain fixed in energy, with the shift in energy of the conduction band minimum causing the states to lie further from the conduction band edge.

\section{Conclusions}

In this chapter, we have investigated $\left(\mathrm{CH}_{3} \mathrm{NH}_{3}\right)_{2} \mathrm{~Pb}(\mathrm{SCN})_{2} \mathrm{I}_{2}$ (MAPSI) and its analogues as potential solar absorber materials. We find that MAPSI possesses an electronic structure suitable for a photovoltaic top cell, with a band gap of $\sim 1.79 \mathrm{eV}$ (albeit slightly underestimated compared to the experimental gap of $\sim 2.1 \mathrm{eV}$ ), small charge-carrier effective masses within the perovskite-type layers, and enhanced stability against decomposition compared with the prototypical hybrid perovskite, $\mathrm{CH}_{3} \mathrm{NH}_{3} \mathrm{PbI}_{3}$ (MAPI). Our results suggest that MAPSI 
maintains a smaller band gap, when compared to other two-dimensional absorbers due to the presence of SCN states at the top of the valence band maximum. Furthermore, its intrinsic defect chemistry shows few deep defect states that could contribute to carrier recombination.

We have further investigated the potential for MAPSI to act as a parent compound to a range of analogues with the same crystal structure, thereby allowing for a material with three degrees of property tuning. All materials in the $\left(\mathrm{CH}_{3} \mathrm{NH}_{3}\right)_{2} \mathrm{MPs}_{2} \mathrm{X}_{2}$ series (where $\mathrm{M}=$ $\mathrm{Pb}$, Sn; Ps = OCN, SCN, SeCN; and X = Cl, Br, I) were found to maintain the MAPSI structural motif, with all showing stability against phase separation. We demonstrated that the electronic properties of the series are dictated primarily by the choice in halide, with several compounds possessing band gaps suitable for photovoltaic applications. Band alignment calculations reveal MAPSI shows a similar ionisation potential and electron affinity to MAPI, indicating that hole and electron contact materials commonly used in the production of hybrid halide devices, will also perform well for MAPSI and its analogues. Overall, the iodide-based analogues show the most promise for solar devices, due to their ideal band gaps and relatively large dielectric constants. Due to concerns over the toxicity of lead, $\left(\mathrm{CH}_{3} \mathrm{NH}_{3}\right)_{2} \mathrm{Sn}(\mathrm{SCN})_{2} \mathrm{I}_{2}$ is a particularly exciting addition to the perovskite family, based on its potential as a stable and efficient lead-free photovoltaic.

\section{Notes}

The work in this chapter formed the basis for two publications:

1. A. M. Ganose, C. N. Savory and D. O. Scanlon, $\left(\mathrm{CH}_{3} \mathrm{NH}_{3}\right)_{2} \mathrm{~Pb}(\mathrm{SCN})_{2} \mathrm{I}_{2}$ : a more stable structural motif for hybrid halide photovoltaics?, Journal of Physical Chemistry Letters, 6, 4594 (2015)

2. A. M. Ganose, C. N. Savory and D. O. Scanlon, $\left(\mathrm{CH}_{3} \mathrm{NH}_{3}\right)_{2} \mathrm{PbI}_{2}(\mathrm{SCN})_{2}$ analogues for photovoltaic applications, Journal of Materials Chemistry A, 5, 7845-7853 (2017)

The optimised crystal structures, for all compounds discussed in this chapter, are provided in an online repository. ${ }^{233}$ 


\section{VACANCY-ORDERED DOUBLE PEROVSKITES}

\section{Introduction}

The tin-based hybrid perovskite, $\mathrm{MASnI}_{3}$, and its inorganic counterpart, $\mathrm{CsSnI}_{3}$, have received significant attention as lead-free alternatives for perovskite photovoltaics. ${ }^{194,198,199,234,235}$ While the efficiencies of devices containing these compounds has reached over $9.0 \%$ in recent years, ${ }^{193}$ their extremely poor long-term stability - worse, even, than their lead analogues - presents a serious challenge that remains unresolved. ${ }^{195,202,203}$ This instability stems from the facile oxidation of $\mathrm{Sn}^{2+}$ to $\mathrm{Sn}^{4+}$, which occurs readily in heat, light or contact with moisture. ${ }^{188,189}$ Accordingly, significant work will be required if these materials are to find viable practical applications. ${ }^{236}$

The broad diversity in composition and structure exhibited by the perovskite family (formula $\mathrm{ABX}_{3}$ ), provides a number of routes to circumvent these issues. For example, it is possible to produce double halide perovskites (with the formula $\mathrm{A}_{2} \mathrm{~B}^{\prime} \mathrm{B}^{\prime \prime} \mathrm{X}_{6}$ ), which show rock-salt ordering of the $\mathrm{B}^{\prime}$ and $\mathrm{B}^{\prime \prime}$ metal cations due to size and charge differences. ${ }^{237-239}$ Unfortunately, recent work has shown most lead-free double perovskites will exhibit indirect band gaps, owing to a mismatch in angular momentum of the frontier electronic orbitals. ${ }^{240}$ While this issue can be avoided through employing indium or thallium, the resulting compounds are either thermodynamically unstable or extremely toxic. ${ }^{240}$

The related vacancy-ordered double perovskites can be produced by replacing one B-site cation with a vacancy, resulting in a material 


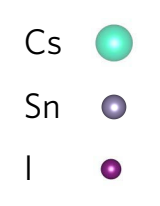

FIGURE 6.1: Crystal structure of $\mathrm{Cs}_{2} \mathrm{Snl}_{6}$ viewed along (a) the [010] direction, (b) a threedimensional view, highlighting the isolated $\left[\mathrm{SnI}_{6}\right]^{2-}$ octahedral units. Sn, I, and Cs atoms are denoted by grey, purple, and turquoise spheres, respectively. Reprinted with permission from Ref. 17.

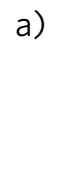

$\stackrel{a}{\longrightarrow} c$
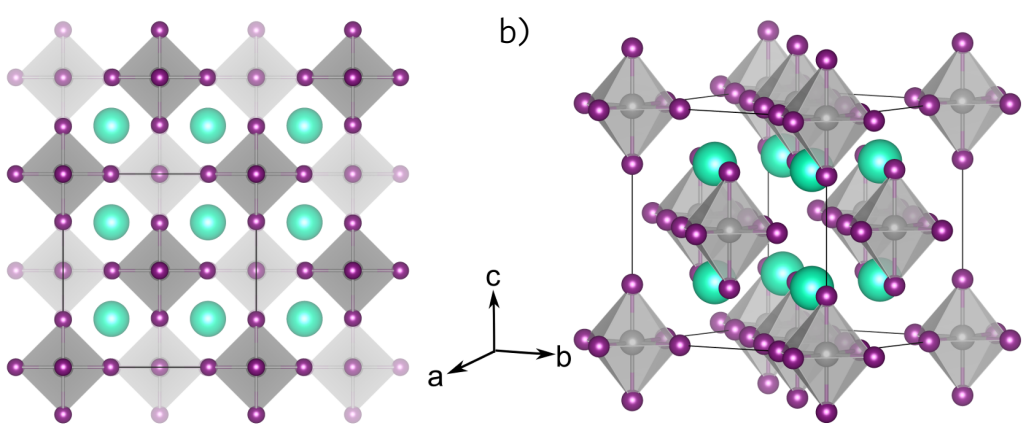

with the $\mathrm{A}_{2} \mathrm{BX}_{6}$ formula (or $\mathrm{A}_{2} \mathrm{~B} \square \mathrm{X}_{6}$, where $\square$ is a vacancy). They crystallise in the $\mathrm{K}_{2} \mathrm{PtCl}_{6}$ structure (termed antifluorite), containing isolated $\left[\mathrm{BX}_{6}\right]^{2-}$ octahedra. The structure is charge balanced by $\mathrm{A}^{+}$ cations residing in the cuboctahedral void, formed by the 12 nearestneighbour X site anions (Fig. 6.1). Despite the loss of every other $\mathrm{B}$-site cation, the $\mathrm{A}_{2} \mathrm{BX}_{6}$ perovskites demonstrate many similar properties to their $\mathrm{ABX}_{3}$ counterparts, in part due to the retention of the close-packed anionic sublattice.

The inorganic $\mathrm{Cs}_{2} \mathrm{SnI}_{6}$ defect-perovskite has recently attracted attention as a stable alternative to $\mathrm{CsSnI}_{3}$. Crystallising in the cubic $F m \overline{3} m$ space group (Fig. 6.1), it possesses many properties ideal for photovoltaics; namely a band gap of $\sim 1.3 \mathrm{eV}$, strong optical absorption over $10^{5} \mathrm{~cm}^{-1}$ at $1.7 \mathrm{eV}$ above the valence band edge, and intrinsic $n$-type electrical conductivity. ${ }^{241,242}$ Furthermore, $\mathrm{Cs}_{2} \mathrm{SnI}_{6}$ shows remarkably improved chemical stability in the presence of moisture and heat (stable up to $270{ }^{\circ} \mathrm{C}$ ), due the formally $4+$ oxidation state of $\mathrm{Sn}$ in the structure. ${ }^{243}$ Remarkably, polycrystalline pellets of $\mathrm{Cs}_{2} \mathrm{SnI}_{6}$ have reportedly achieved carrier mobilities comparable to that of $\mathrm{CsSnI}_{3}$ ( $\mu=310$ and $585 \mathrm{~cm}^{2} \mathrm{~V}^{-1} \mathrm{~s}^{-1}$, respectively). ${ }^{195,241} \mathrm{~A}$ recent report on $\mathrm{Cs}_{2} \mathrm{SnI}_{6}$ thin films observed more modest electron mobilities of $3 \mathrm{~cm}^{2} \mathrm{~V}^{-1} \mathrm{~s}^{-1}$, however, these results are still surprising considering the presence of regular B-site vacancies. ${ }^{242}$ While a thorough understanding of the origins of conductivity in $\mathrm{Cs}_{2} \mathrm{SnI}_{6}$ remains limited, the formation of donor iodine vacancy defects, in addition to small electron effective masses, has been proposed. ${ }^{241,242,244}$

The first devices containing the vacancy-ordered perovskites employed $\mathrm{Cs}_{2} \mathrm{SnI}_{6}$ as the hole transporting layer for dye-sensitised solar cells, achieving power conversion efficiencies of $\sim 8 \%{ }^{241,245} \mathrm{Cs}_{2} \mathrm{SnI}_{6}$ 
was subsequently incorporated as the absorber layer on $\mathrm{TiO}_{2}$ and $\mathrm{ZnO}$ nanorods, achieving efficiencies of $0.96 \%$ and $0.86 \%$, respectively. ${ }^{246,247}$ Work by Lee et al. on optimising the crystallinity of solutionprocessed $\mathrm{Cs}_{2} \mathrm{SnI}_{6}$ thin films, further enabled devices with efficiencies up to $1.47 \%{ }^{248}$ The authors additionally explored members of the solid solution, $\mathrm{Cs}_{2} \mathrm{SnI}_{x} \mathrm{Br}_{6-x}$, allowing for cells with efficiencies over $2.0 \%$ (for the composition $\mathrm{Cs}_{2} \mathrm{SnI}_{4} \mathrm{Br}_{2}$ ), due to enhanced opencircuit voltage. Recent developments in producing $\mathrm{Cs}_{2} \mathrm{SnI}_{6}$ nanocrystals with tuneable optoelectronic properties, including effective masses a factor of 4 smaller than the bulk material, highlight the potential for further advancements in device engineering. ${ }^{249}$

\section{$\mathrm{Cs}_{2} \mathrm{Sn}_{1-x} \mathrm{Te}_{x} \mathrm{I}_{6}$ solid solutions}

A proposed route to enhancing the carrier mobility of $\mathrm{Cs}_{2} \mathrm{SnI}_{6}$ is through substitution of $\mathrm{Sn}^{4+}$ (electron configuration: [Kr] $4 d^{10} 5 s^{0}$ ) with $\mathrm{Te}^{4+}$ (electron configuration: $[\mathrm{Kr}] 4 d^{10} 5 s^{2}$ ). $\mathrm{Cs}_{2} \mathrm{TeI}_{6}$ is isostructural with $\mathrm{Cs}_{2} \mathrm{SnI}_{6}$, however, the larger $\mathrm{Te}^{4+}$ is accommodated into the lattice through expansion of the $[\mathrm{BI}]_{6}$ octahedra, reducing the volume of the inter-octahedral void. As the valence and conduction band edges are dominated by iodine character, the increased inter-octahedral I-I orbital overlap is expected to result in greater band dispersion and smaller effective masses. High-resolution synchrotron powder X-ray diffraction (SXRD) for the $\mathrm{Cs}_{2} \mathrm{Sn}_{1-x} \mathrm{Te}_{x} \mathrm{I}_{6}$ series, performed by our collaborators, Maughan et al., at Colorado State University, indicates tellurium substitution results in solid so-
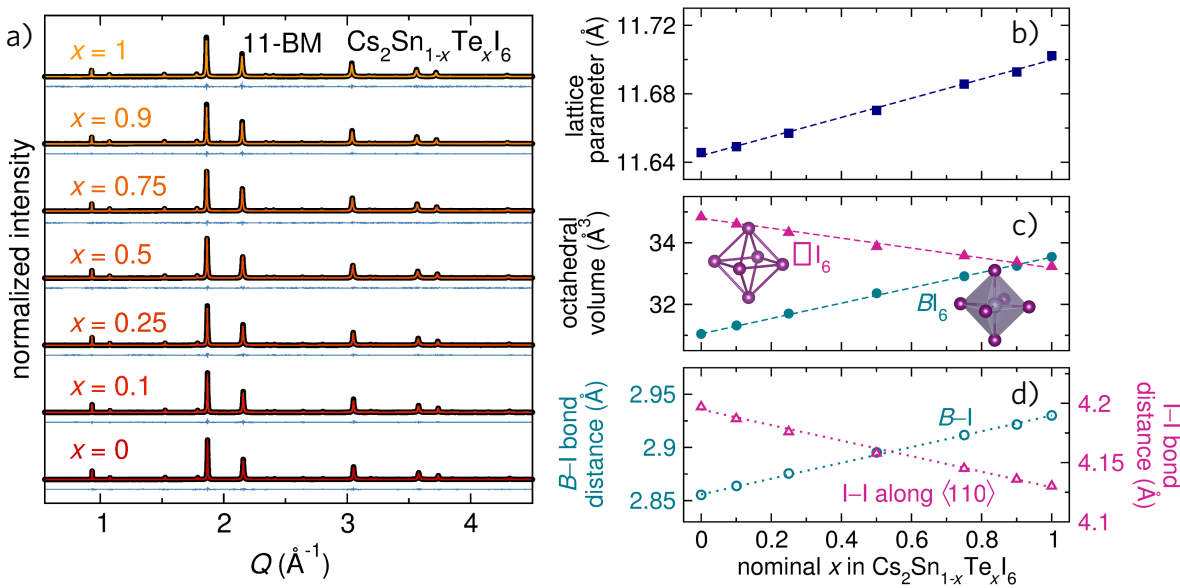

FIGURE 6.2: Structural data for the solid-solution series, $\mathrm{Cs}_{2} \mathrm{Sn}_{1-x} \mathrm{Te}_{x} \mathrm{I}_{6}$. a) Highresolution synchrotron $\mathrm{X}$-ray diffraction data and Rietveld refinements, collected at room temperature. Black circles indicate collected data, coloured lines indicate the fit, and blue lines are the difference curves. b-d) Structure parameters across the series, determined from high-resolution synchrotron X-ray diffraction. b) The refined lattice parameters. Vegard's law interpolated from the end members is shown by a dashed blue line. c) Average polyhedral volumes of the $\square \mathrm{I}_{6}$ voids and $\mathrm{BI}_{6}$ octahedra. As the volume of the $\mathrm{BI}_{6}$ octahedra increase, the volume of the void regions decrease. d) Average I - I and B - I bond distances, along the [110] direction. Dashed lines in (d) and (c) represent linear regressions. Data collected and plotted by Maughan et al. ${ }^{17}$ 
FIGURE 6.3: Optoelectronic properties for the series $\mathrm{Cs}_{2} \mathrm{Sn}_{1-x} \mathrm{Te}_{x} \mathrm{I}_{6}$. a) Dependence of the electrical resistivity on temperature. b) Room temperature resistivity. c) Carrier mobility ( $\mu_{\mathrm{e}}$, open pink diamonds) and carrier concentration ( $n_{\mathrm{e}}$, filled blue triangles). d) The optical band gap, derived from UV-visible diffuse reflectance data. Data collected and plotted by Maughan et al. ${ }^{17}$

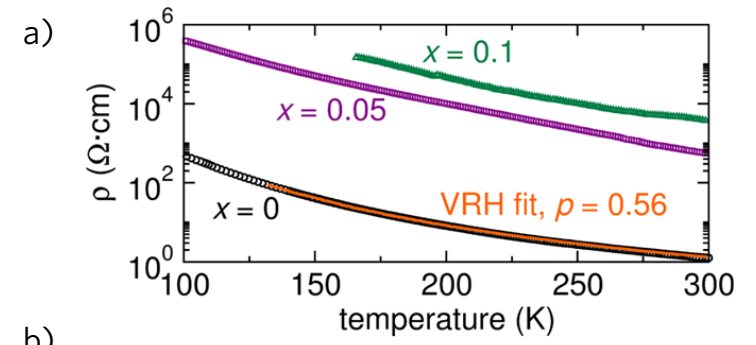

b)
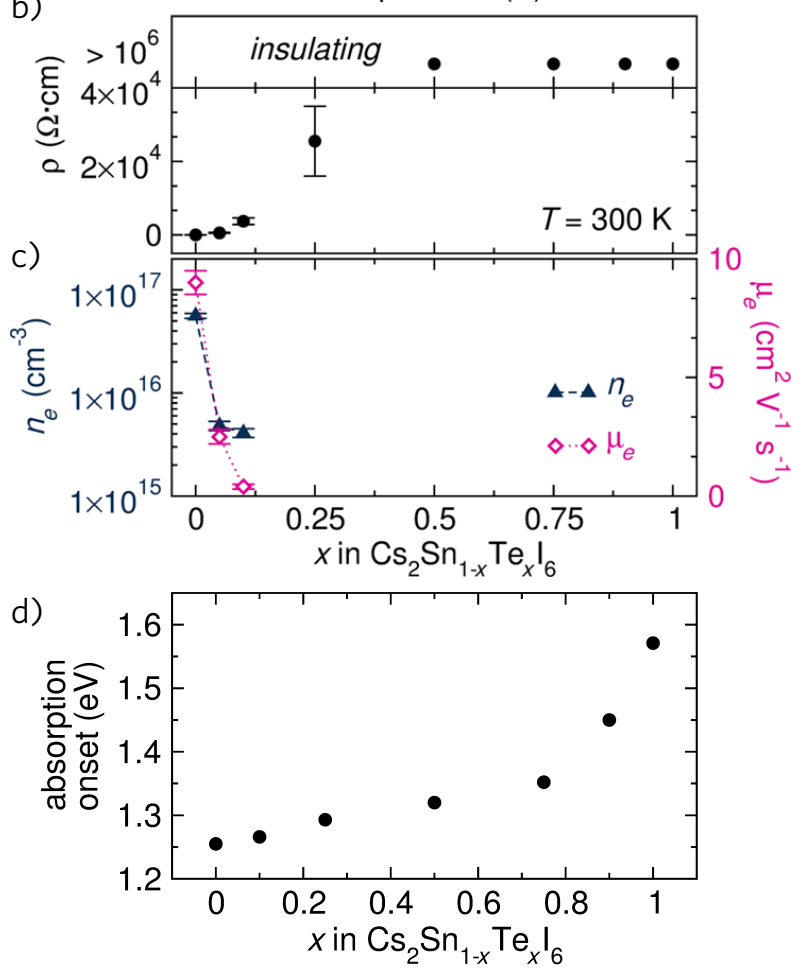

lution behaviour, with no distortion in the local coordination environment of each octahedron (Fig. $6.2 a) .{ }^{17}$ The series shows a linear increase of lattice parameter upon tellurium substitution, following Vegards law, with a simultaneous decrease in interoctahedral I- I bond lengths (Fig. 6.2d).

As previously discussed, $\mathrm{Cs}_{2} \mathrm{SnI}_{6}$ is an intrinsic $n$-type semiconductor. This was confirmed by measurements performed by our collaborators, who found a resistivity of $13 \Omega \mathrm{cm}$ for cold-pressed polycrystalline pellets at $\mathrm{T}=300 \mathrm{~K}$, with Hall measurements indicating carrier concentrations of $5 \times 10^{16} \mathrm{~cm}^{-3}$ and an electron mobilities of $8.6 \mathrm{~cm}^{2} \mathrm{~V}^{-1} \mathrm{~s}^{-1} .{ }^{17}$ Unexpectedly, partial substitution of tin by tellurium, to form the solid solution $\mathrm{Cs}_{2} \mathrm{Sn}_{1-x} \mathrm{Te}_{x} \mathrm{I}_{6}$, resulted in a significant increase in electrical resistivity (Fig. 6.3a). Substitution of $5 \%$ tellurium resulted in a 50 -fold increase in resistivity, with samples contain- 
ing more than $25 \%$ tellurium found to be too insulating for accurate measurements. Hall measurements of the $5 \%$ and $10 \%$ tellurium members of the solid solutions further revealed dramatically decreased carrier concentrations and electron mobilities (Fig. 6.3b). Maughan et al. also observed a nonlinear change in the measured optical band gap upon tellurium substitution (Fig. 6.3d), despite the absence of any distortions in the local geometry, as determined by X-ray pair distribution function analysis. ${ }^{17}$ As such, it is clear that a more detailed understanding of the electronic, optical, and defect properties is essential to shed light on the behaviour of the series.

\section{Methodology}

Calculations were performed using the Vienna $A b$ initio Simulation Package. A k-point mesh of $\Gamma$-centred $3 \times 3 \times 3$ and plane wave cutoff of $350 \mathrm{eV}$ was found to converge the 9 atom primitive cells of $\mathrm{Cs}_{2} \mathrm{SnI}_{6}$ and $\mathrm{Cs}_{2} \mathrm{TeI}_{6}$ to within $1 \mathrm{meV}$ /atom. During geometry optimisations, the cutoff was increased to $455 \mathrm{eV}$ to avoid errors resulting from $\mathrm{Pu}$ lay stress. ${ }^{85}$ The structures were deemed converged when the forces totalled less than $10 \mathrm{meV}^{-1}$.

The HSE06 functional was employed for geometry relaxations. Electronic properties were calculated using HSE06 with the addition of spin-orbit coupling effects (HSE06+SOC). The Brillouin zone for the $F m \overline{3} m$ space group, indicating the high-symmetry points explored in the band structure, is provided in Fig. 6.4. project crystal orbital Hamilton population (pCOHP) analysis was performed using the LOBSTER program, based on wavefunctions calculated using the HSE06 functional. ${ }^{92,95}$

For band alignment calculations, the core-level alignment approach of Wei and Zunger was employed, ${ }^{217}$ using a slab model with $35 \AA$ of vacuum and a $45 \AA$ thick slab. The slab was cleaved along the non-polar (101) surface, due to the absence of any dangling bonds. Due to the size of the model, which precluded the use of HSE06+SOC, band alignment calculations were performed using HSE06, with an explicit correction to the band gap and valence band maximum po-

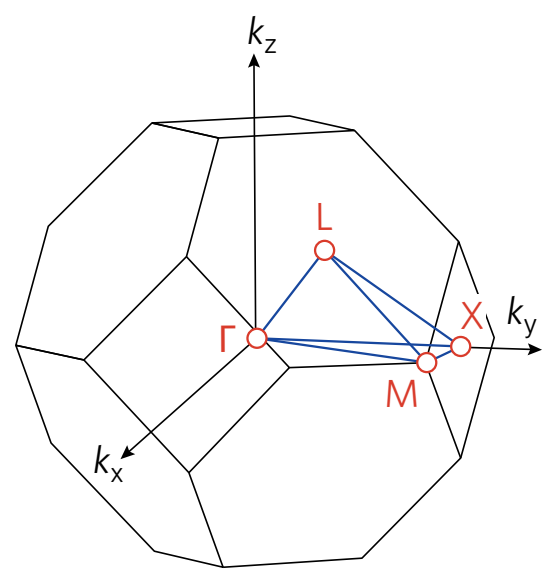

FIGURE 6.4: Brillouin zone of the $F m \overline{3} m$ space group. Coordinates of the high symmetry points used for the band structures and effective masses: $\Gamma=(0,0,0) ; X=$ $(1 / 2,0,1 / 2) ; L=(1 / 2,1 / 2,1 / 2) ; W=$ $(1 / 2,1 / 4,3 / 4)$. 
TABLE 6.1: HSEO6 calculated lattice parameters and bond lengths for the conventional cells of $\mathrm{Cs}_{2} \mathrm{Snl}_{6}$ and $\mathrm{Cs}_{2} \mathrm{Tel}_{6}$. The lattice parameters and cation-anion interatomic distances are quoted in $\AA$. The equilibrium crystal structures can be found online in a public repository 251 sition taken from the HSE06+SOC calculated bulk.

Defect calculations were performed in a $3 \times 3 \times 3$ supercell containing 243 atoms, using the HSE06 functional and a single $\mathbf{k}$-point at $\Gamma$. The defect energies were corrected to account for use of a finitesized supercell, using the potential level alignment, band-filling and image-charge corrections described in Chapter 3.

\section{Results}

\section{Geometric structure}

The lattice constants and structural parameters of $\mathrm{Cs}_{2} \mathrm{SnI}_{6}$ and $\mathrm{Cs}_{2} \mathrm{TeI}_{6}$, calculated using HSE06, are provided in Table 6.1. The results are in keeping with previous studies performed using HSE06. ${ }^{244,250}$ The lattice parameters are slightly overestimated compared to experiment, by $\sim 2 \%$ in both cases. Moving from $\mathrm{Cs}_{2} \mathrm{SnI}_{6}$ to $\mathrm{Cs}_{2} \mathrm{TeI}_{6}$, the calculated lattice parameter increases by $0.02 \AA$; this is in good agreement to experiment, which shows a $0.05 \AA$ increase in the $a$ parameter. Furthermore, the change in the calculated B-I and I-I bond distances ( $0.08 \AA$ in both cases), agrees well with the change seen in experiment ( 0.07 in both cases).

\begin{tabular}{lcccc}
\hline Compound & $a$ & $d_{\mathrm{B}-\mathrm{I}}$ & $d_{\mathrm{B}-\mathrm{Cs}}$ & $d_{\mathrm{I}-\mathrm{I}}$ \\
\hline $\mathrm{Cs}_{2} \mathrm{SnI}_{6}$ & 11.96 & 2.86 & 5.18 & 4.40 \\
$\mathrm{Cs}_{2} \mathrm{TeI}_{6}$ & 11.98 & 2.94 & 5.19 & 4.32 \\
\hline
\end{tabular}

\section{Electronic properties}

Some debate exists as to the nature and magnitude of the fundamental band gap in $\mathrm{Cs}_{2} \mathrm{SnI}_{6}$. Two previous experimental studies have reported a gap of $1.3 \mathrm{eV}, 242,252$ but differ on whether it is direct or indirect; whereas work on $\mathrm{Cs}_{2} \mathrm{SnI}_{6}$ thin films, suggested a direct gap of $1.6 \mathrm{eV} .{ }^{241}$ The analysis performed by our co-workers, Maughan et al., suggests an optical gap of $\sim 1.25 \mathrm{eV}$ for $\mathrm{Cs}_{2} \mathrm{SnI}_{6} \cdot{ }^{17}$ Comparable analysis for $\mathrm{Cs}_{2} \mathrm{TeI}_{6}$, indicates an optical gap of $\sim 1.59 \mathrm{eV},{ }^{17}$ in agreement with the previously reported indirect gap of $1.5 \mathrm{eV} .{ }^{253}$ 

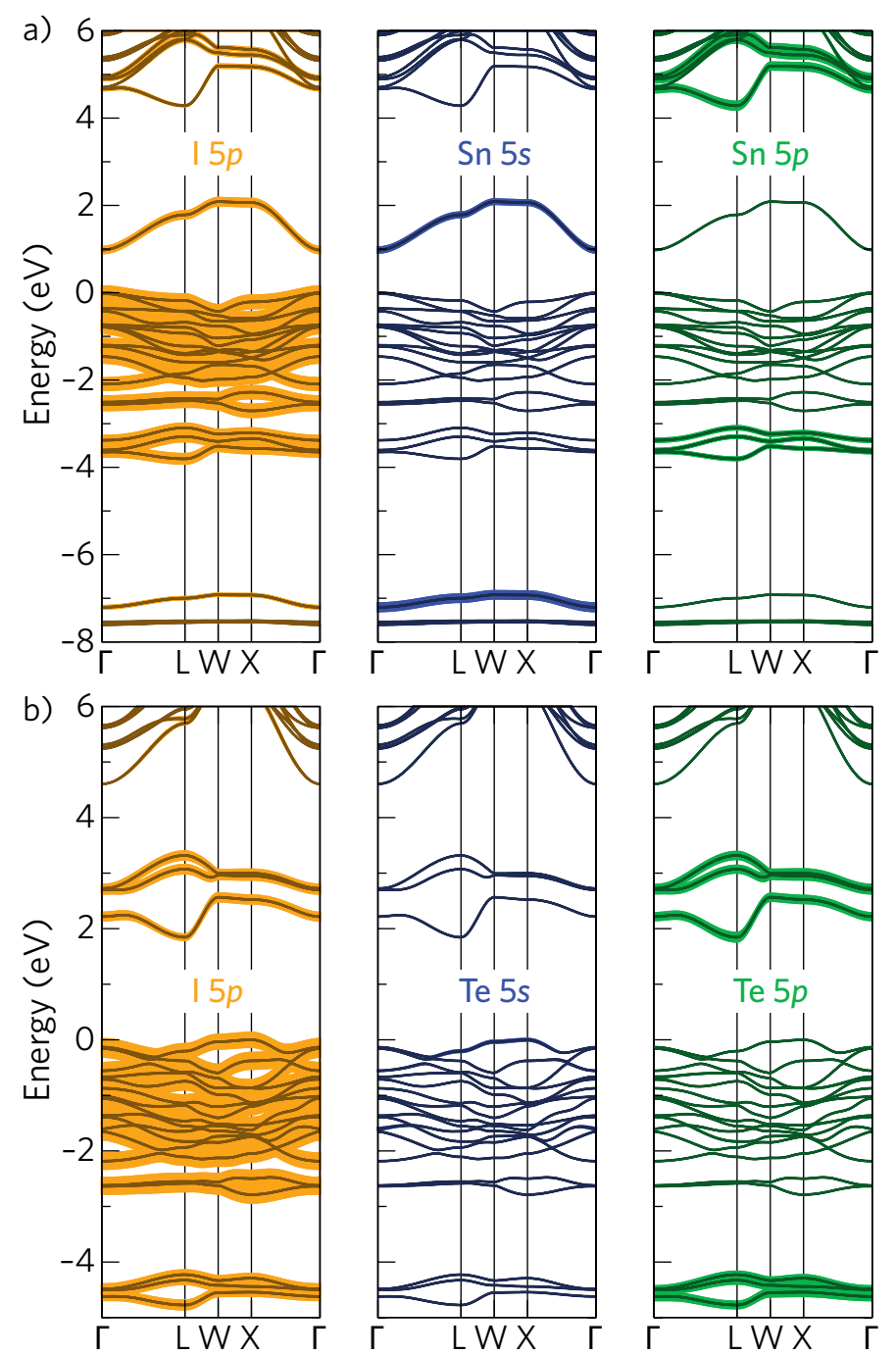

The band structures of $\mathrm{Cs}_{2} \mathrm{SnI}_{6}$ and $\mathrm{Cs}_{2} \mathrm{TeI}_{6}$, calculated using HSE06 +SOC, are provided in Figure 6.5. The band structure of $\mathrm{Cs}_{2} \mathrm{SnI}_{6}$ confirms it is a direct gap semiconductor, with a fundamental band gap of $0.97 \mathrm{eV}$ occurring at the $\Gamma$ point. This result is in reasonable agreement with GW0 calculations, which indicate a direct gap of $0.88 \mathrm{eV} .{ }^{245}$ We note that the calculated band gap is noticeably smaller than the optical band gap seen in experiment $(\sim 1.3 \mathrm{eV})-$ a point to which we will return later. The band structure of $\mathrm{Cs}_{2} \mathrm{TeI}_{6}$ replicates experimental observations of an indirect band gap, however the calculated gap $\left(\mathrm{E}_{\mathrm{g}}^{\mathrm{ind}}=1.83 \mathrm{eV}\right)$ is larger than the experimental optical gap $(1.59 \mathrm{eV})$. As observed in other tellurium-based materials, this difference may result from many-body effects that require higher order methods, such as configuration interactions, to correctly de-
FIGURE 6.5: Orbital projected band structures of a) $\mathrm{Cs}_{2} \mathrm{Snl}_{6}$ and b) $\mathrm{Cs}_{2} \mathrm{Tel}_{6}$, calculated using HSEO6+SOC. The contributions from the I $5 p, \mathrm{Sn} / \mathrm{Te} 5 \mathrm{~s}$, and $\mathrm{Sn} / \mathrm{Te}$ $5 p$ are shown by orange, blue, and green, respectively. The valence band maximum is set to $0 \mathrm{eV}$ in all cases. 


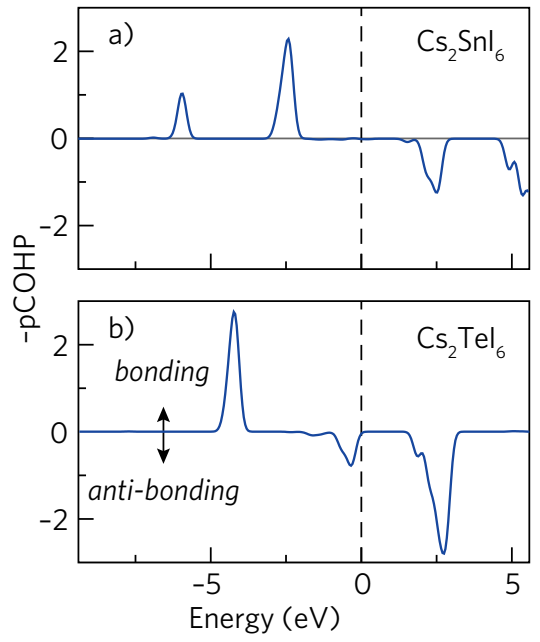

FIGURE 6.6: Projected crystal orbital Hamilton population (pCOHP) analysis of (a) $\mathrm{Cs}_{2} \mathrm{Snl}_{6}$ and (b) $\mathrm{Cs}_{2} \mathrm{Tel}_{6}$, in which the density of states is partitioned with the sign denoting bonding or anti-bonding character, and the magnitude indicating the strength of the interaction. For each compound the pCOHP is averaged across all pairwise $B-I$ interactions in the primitive cell. The valence band maximum is set to $0 \mathrm{eV}$. scribe. ${ }^{254-257}$ The conduction band minimum and valence band maximum of $\mathrm{Cs}_{2} \mathrm{TeI}_{6}$ occur at the $\mathrm{X}$ and $\mathrm{L}$ high-symmetry points, respectively, with the direct band gap $\left(\mathrm{E}_{\mathrm{g}}^{\mathrm{dir}}=2.05 \mathrm{eV}\right)$ appearing at $\mathrm{L}$. The effect of spin-orbit coupling on the electronic properties of $\mathrm{Cs}_{2} \mathrm{SnI}_{6}$ and $\mathrm{Cs}_{2} \mathrm{TeI}_{6}$ was tested, with the full results provided in Appendix $B$. In both cases, the relativistic renormalisation of the band gap occurs through raising of the valence band maximum by $\sim 0.2 \mathrm{eV}$, with the conduction band remaining largely unaffected. The magnitude of this renormalisation indicates that inclusion of spin orbit effects is essential to accurately model these systems.

The band structures presented in Figure 6.5, further include information on the orbital character of the bands. Both $\mathrm{Cs}_{2} \mathrm{SnI}_{6}$ and $\mathrm{Cs}_{2} \mathrm{TeI}_{6}$ possess an upper valence band composed of I $5 p$ states. In $\mathrm{Cs}_{2} \mathrm{SnI}_{6}$, the frontier valence orbitals are nonbonding, whereas for $\mathrm{Cs}_{2} \mathrm{TeI}_{6}$ there is a mixture of antibonding ( $\mathrm{Te} 5 s-\mathrm{I} 5 p$ ) and nonbonding states, as determined by projected crystal orbital Hamilton population (pCOHP) analysis (Fig. 6.6). The conduction band minimum of $\mathrm{Cs}_{2} \mathrm{SnI}_{6}$ is comprised of antibonding I $5 p$ and $\mathrm{Sn} 5 s$ states. In contrast, the lowest unoccupied band of $\mathrm{Cs}_{2} \mathrm{TeI}_{6}$ is formed of antibonding I $5 p$ and Te $5 p$ states. From analysis of the band structure and pCOHP data, it is apparent that the band forming the lower conduction band in $\mathrm{Cs}_{2} \mathrm{SnI}_{6}$ becomes formally occupied upon moving to $\mathrm{Cs}_{2} \mathrm{TeI}_{6}$ due to the presence of the 2 extra electrons provided by Te. The next highest states above the conduction band minimum in $\mathrm{Cs}_{2} \mathrm{SnI}_{6}$ (found at around $4.5 \mathrm{eV}$ ) appear to be shifted down in energy to form the lower conduction band in $\mathrm{Cs}_{2} \mathrm{TeI}_{6}$.

\section{Origin of occupied Sn s electrons}

As observed in other hybrid DFT work on $\mathrm{Cs}_{2} \mathrm{SnI}_{6}$, we note the presence of occupied Sn $5 s$ states $\sim 7 \mathrm{eV}$ beneath the valence band edge (Fig. 6.5a). ${ }^{244,250}$ The existence of these states, despite the presence of $\mathrm{Sn}$ in the 4+ oxidation state, has been the topic of much interest in the literature. ${ }^{250,258}$ Indeed, based on the electronic configuration of $\mathrm{Sn}^{4+}\left(s^{0} p^{0}\right)$, one might expect to only see unoccupied $s$ and $p$ contributions in the conduction band. 
Comparison to the analogous three-dimensional perovskite, $\mathrm{CsSnI}_{3}$, provides insight into the origin of this behaviour. Moving from $\mathrm{CsSnI}_{3}$ to $\mathrm{Cs}_{2} \mathrm{SnI}_{6}$, the introduction of ordered $\mathrm{Sn}$ vacancies results in the effective addition of 2 holes per formula unit. Recent ${ }^{119} \mathrm{Sn}$-Mössbauer spectroscopy and K-edge X-ray spectroscopy measurements, reveal the $\mathrm{Sn} 5 s$ charge distribution is reduced going from $\mathrm{CsSnI}_{3}$ to $\mathrm{Cs}_{2} \mathrm{SnI}_{6}$, as expected based on a formal oxidation state model. ${ }^{258}$ The loss in the Sn $5 s$ electrons, however, is accompanied by an increase in the charge density of the Sn $5 p$ electrons, thereby acting to replenish most of the charge lost. This observation is confirmed by hybrid DFT calculations, which show that the transformation from $\mathrm{CsSnI}_{3}$ to $\mathrm{Cs}_{2} \mathrm{SnI}_{6}$, results in a loss of $0.6 \mathrm{Sn} s$ electrons but concomitant gain of $0.2 p$ electrons. ${ }^{258}$ Accordingly, the total change in the physical charge surrounding the $S n$ atom is just 0.4 electrons, significantly less than expected. It has previously been suggested that the observation of occupied $S n s$ states arises from the presence of $S n$ in the +2 oxidation state. ${ }^{250}$ The calculations described above, however, dispute this picture, proving instead that the Sn $s$ orbitals are half occupied. As such, Dalpian et al. have proposed this behaviour can be explained through a self-regulating response mechanism. ${ }^{258,259}$

This mechanism can be understood by considering a simplified orbital bonding picture for both compounds (Fig. 6.7). The covalent bonding in the $\mathrm{SnI}_{6}$ octahedral complex is dictated by symmetry constraints: specifically, the Sn possesses spherical $s$ states and dumbbell $p$ states, whereas the $\mathrm{I}_{6}$ cage ( $O_{h}$ point group) shows $p$ orbitals split into several symmetry adapted linear combinations. The $\mathrm{Sn} 5 \mathrm{~s}$ electrons can only hybridise with I $5 p A_{1 g}$ states, while the Sn $5 p$ states must couple with the I $5 p T_{1 u}$ states. The remaining I $p$ states, with $E_{g}, T_{1 g}, T_{2 g}$, and $T_{2 u}$ symmetries have no compatible $S n$ states to bond with and will therefore be nonbonding.

In $\mathrm{CsSnI}_{3}$, both the $\mathrm{Sn} 5 s-\mathrm{I} 5 p A_{1 g}$ bonding and antibonding orbitals are occupied, resulting in a fully occupied $\mathrm{Sn} 5 s$ shell (Fig. 6.7a). In contrast, the Sn $5 p-\mathrm{I} 5 p T_{1 u}$ bonding orbital is occupied, whereas the antibonding is not, resulting in a band gap transition from the antibonding $A_{1 g}$ states to the antibonding $T_{1 u}$ states. Moving from 
a)

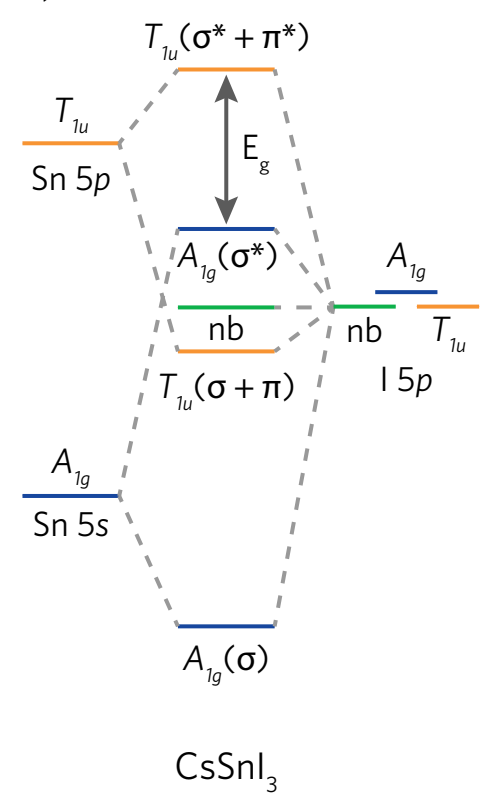

b)

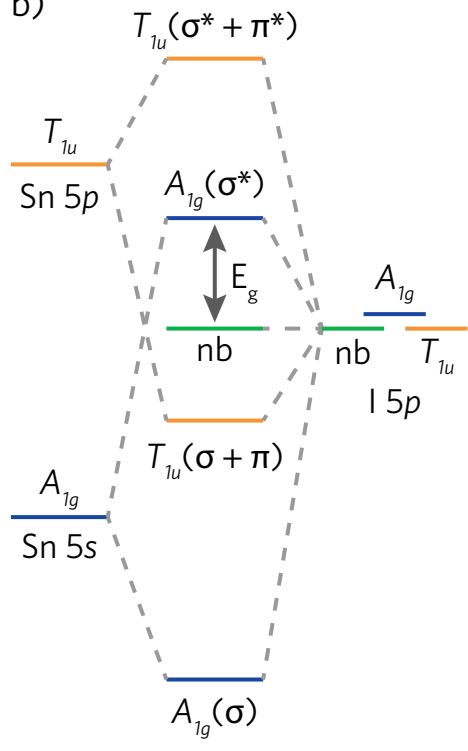

$\mathrm{Cs}_{2} \mathrm{SnI}_{6}$

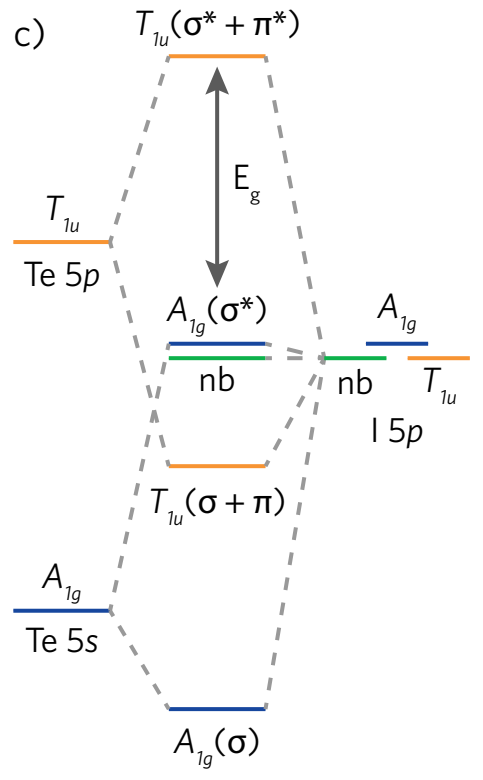

$\mathrm{Cs}_{2} \mathrm{Tel}_{6}$
FIGURE 6.7: Simplified molecular orbital picture of a) $\mathrm{Snl}_{6}$ in $\mathrm{CsSnl}_{3}$, b) $\mathrm{Snl}_{6}$ in $\mathrm{Cs}_{2} \mathrm{SnI}_{6}$, and c) $\mathrm{Tel}_{6}$ in $\mathrm{Cs}_{2} \mathrm{Tel}_{6} . A_{1 g}, \mathrm{~T}_{1 u}$, and nonbonding $(n b)$ orbitals indicated by blue, orange, and green lines, respectively.
$\mathrm{CsSnI}_{3}$ to $\mathrm{Cs}_{2} \mathrm{SnI}_{6}$, the introduction of two holes (due to the regular Sn-site vacancies), causes the Fermi level to shift down, thereby depopulating the antibonding $A_{1 g}$ states (Fig. 6.7b). As these states contain greater I $5 p$ than $\mathrm{Sn} 5 p$ character, the holes are disproportionately distributed over the $\mathrm{I}_{6}$ octahedra, accounting for the smaller than expected 0.6 electron loss in the Sn $s$ density. The transition from $\mathrm{CsSnI}_{3}$ to $\mathrm{Cs}_{2} \mathrm{SnI}_{6}$, is accompanied by a $8 \%$ reduction in the bond lengths of the $\mathrm{SnI}_{6}$ octahedra, resulting in increased $\mathrm{Sn}-\mathrm{I}$ orbital coupling. ${ }^{258}$ This acts to push the bonding Sn $5 p-\mathrm{I} 5 p T_{1 u}$ states lower in energy, while simultaneously increasing their Sn $5 p$ character. Accordingly, the greater hybridisation results in an increase of occupied Sn $5 p$ states, providing an explanation for the greater Sn $p$ density seen in experiment. The remaining holes are instead localised in a complex arrangement throughout the iodine close-packed lattice, as indicated by charge density isosurfaces. ${ }^{258}$ This subtle feedback loop - namely, greater occupation of the bonding Sn $5 p$ orbitals upon depopulation of the antibonding Sn $5 s$ states - effectively acts to minimise the effects of charge perturbation caused by vacancy formation and has been termed a self-regulating response. ${ }^{258,259}$

The molecular orbital picture can be extended to understand the behaviour of $\mathrm{Cs}_{2} \mathrm{TeI}_{6}$. The $5 s^{2} p^{0}$ electron configuration of $\mathrm{Te}^{4+}$, effectively adds two electrons per formula unit, thereby repopulating the 
$A_{1 g}$ antibonding orbitals depopulated in the transition from $\mathrm{CsSnI}_{3}$ to $\mathrm{Cs}_{2} \mathrm{SnI}_{6}$ (Fig. 6.7c). Due to the higher binding energy of the Te $5 s$ orbitals (in comparison to the Sn 5s), these states occur further away in energy from the I $5 p$ states. This results in a significant reduction in hybridisation, causing the antibonding $A_{1 g}$ orbitals to sit very close in energy to the nonbonding I $5 p$ states. This is confirmed by the orbital projected band structures, which show the majority of the Te $5 s$ states localised around $12 \mathrm{eV}$ beneath the valence band maximum, with only a small number of states present at the band edge. In contrast, the larger binding energy of the Te $5 p$ states brings them closer in energy to the I $5 p$, increasing the hybridisation and raising the antibonding $T_{1 u}$ states. Combined, this behaviour has the effect of significantly raising the band gap of $\mathrm{Cs}_{2} \mathrm{TeI}_{6}$ versus $\mathrm{Cs}_{2} \mathrm{SnI}_{6}$.

\section{Nature of the $\mathrm{Cs}_{2} \mathrm{SnI}_{6}$ band gap}

In order to investigate the difference between the direct fundamental band gap and the experimentally observed optical band gap of $\mathrm{Cs}_{2} \mathrm{SnI}_{6}$, we have calculated the transition matrix elements for the transitions between the valence and conduction band states, using HSE06+SOC. The transitions were categorised as dipole allowed or disallowed, based on the magnitude of the matrix element square $\left(|M|^{2}\right)$. Specifically, a transition was deemed allowed if $|M|^{2}>10^{-3} \mathrm{eV}^{-2} \AA^{-2}$, else it was considered dipole forbidden, as described in Ref. Yu and Zunger ${ }^{12}$. Analysis of the direct valence band to conduction band transitions, indicates that photo-excitation from the twofold-degenerate valence band maximum to the conduction band minimum at $\Gamma\left(A_{1 g}\right.$ symmetry), is dipole forbidden. This effect is well known in materials whose crystal structures possess a centre of inversion ${ }^{260,261}$ and results as strong optical transitions are only permitted between states of opposing parity. Accordingly, the nonbonding states I $5 p$ states at the top of the valence band must possess either $E_{g}, T_{1 g}$, or $T_{2 g}$ symmetry. Transitions from the doubly degenerate bands $0.02 \mathrm{eV}$ beneath the valence band maximum were also found to be dipole forbidden. It is only from $0.35 \mathrm{eV}$ below the valence band maximum that strong transitions are observed, as illustrated in Figure 6.8. These bands 


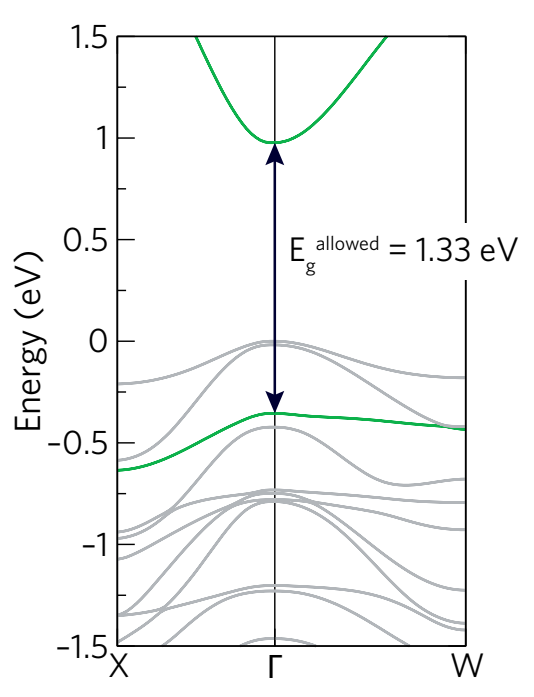

FIGURE 6.8: Band structure of $\mathrm{Cs}_{2} \mathrm{Snl}_{6}$, calculated using HSEO6+SOC. The bands highlighted in green, indicate the states involved in the allowed optical band gap at $\Gamma$. The valence band maximum is set to $0 \mathrm{eV}$. must therefore be the nonbonding I $5 p$ states with $T_{2 u}$ symmetry. As such, the onset of optical absorption occurs at $1.32 \mathrm{eV}$, considerably larger than the direct band gap of $0.97 \mathrm{eV}$ and in much better agreement with the experimental optical band gap of $1.26 \mathrm{eV}$. Accordingly, we believe that previous studies have erroneously employed large amounts of HF exchange ( $\alpha$ parameter in the HSE functional) in order to mistakenly fit the fundamental band gap to the experimental optical band gap. ${ }^{242,244,250}$

The symmetry forbidden fundamental band gap provides a possible explanation for the non-linear trend in optical gaps seen across the solid-solution series, $\mathrm{Cs}_{2} \mathrm{Sn}_{1-x} \mathrm{Te}_{x} \mathrm{I}_{6}$. As demonstrated in experiments performed by our collaborators, Maughan et al., ${ }^{17}$ tellurium incorporation only results in a slight increase in the optical band gap between $x=0$ and 0.75 , after which a steep rise is observed (see Fig. 6.3d). It is possible that the introduction of small amounts of Te will break the symmetry of the frontier bonding states, causing the fundamental band gap to become weakly dipole allowed. In this way, the rise in the band gap expected upon Te substitution is offset by greater absorption occurring from the band edges. When the fundamental band gap becomes fully allowed, a sharper increase in the absorption will be observed, as now the band gap is solely dictated by the rise in the antibonding $T_{1 u}$ states and lowering of the antibonding $A_{1 g}$ states (as discussed in the previous section).

\section{Effective masses}

The effective masses of $\mathrm{Cs}_{2} \mathrm{SnI}_{6}$ and $\mathrm{Cs}_{2} \mathrm{TeI}_{6}$ are summarised in Table 6.2. The electron effective masses $\left(m_{\mathrm{e}}^{*}\right)$ of $\mathrm{Cs}_{2} \mathrm{SnI}_{6}$ were found to be relatively small, with masses of $0.33 \mathrm{~m}_{0}$ seen for both the $\Gamma \rightarrow \mathrm{X}$ (along [ $\left[\begin{array}{ll}1 & 0\end{array}\right]$ ) and $\Gamma \rightarrow \mathrm{L}$ (along [0.5 0.50 .5$]$ ) directions. The effective masses of $\mathrm{Cs}_{2} \mathrm{TeI}_{6}$ show greater anisotropy, with masses of $0.45 \mathrm{~m}_{0}$ and $0.30 \mathrm{~m}_{0}$ seen for the $\Gamma \rightarrow \mathrm{L}$ and $\mathrm{W} \rightarrow \mathrm{L}$ (along [ $\left[\begin{array}{lll}0 & 0.5 & -0.5\end{array}\right]$ ) directions, respectively. We note, that while there are slight differences in the effective masses between the two compounds, these are insufficient to account for the dramatic reduction in carrier mobilities seen in experiment when moving from $\mathrm{Cs}_{2} \mathrm{SnI}_{6}$ to $\mathrm{Cs}_{2} \mathrm{TeI}_{6}$, as 
discussed in Section 6.1.1. The masses are slightly larger than those seen in the three-dimensional hybrid perovskites, $\mathrm{CH}_{3} \mathrm{NH}_{3} \mathrm{PbI}_{3}$ and $\mathrm{CH}_{3} \mathrm{NH}_{3} \mathrm{SnI}_{3}$, which show electron effective masses of $0.15 \mathrm{~m}_{0}$ and $0.28 \mathrm{~m}_{0}$, respectively. ${ }^{22,188}$

\begin{tabular}{lcccccccc}
\hline & \multicolumn{4}{c}{$m_{\mathrm{e}}^{*}$} & & \multicolumn{3}{c}{$m_{\mathrm{h}}^{*}$} \\
\cline { 2 - 4 } \cline { 7 - 8 } Compound & $\Gamma \rightarrow \mathrm{X}$ & $\Gamma \rightarrow \mathrm{L}$ & $\mathrm{W} \rightarrow \mathrm{L}$ & & $\Gamma \rightarrow \mathrm{X}$ & $\Gamma \rightarrow \mathrm{L}$ & $\mathrm{W} \rightarrow \mathrm{L}$ \\
\hline $\mathrm{Cs}_{2} \mathrm{SnI}_{6}$ & 0.33 & 0.33 & - & & 0.96 & 0.93 & - \\
$\mathrm{Cs}_{2} \mathrm{TeI}_{6}$ & - & 0.45 & 0.30 & & 0.65 & - & 3.00 \\
\hline
\end{tabular}

In contrast to the hybrid perovskites, in which the hole effective masses $\left(m_{\mathrm{h}}^{*}\right)$ are smaller than the electron effective masses $\left(m_{\mathrm{h}}^{*}\right.$ $=0.12 \mathrm{~m}_{0}$ and $0.13 \mathrm{~m}_{0}$, for $\mathrm{CH}_{3} \mathrm{NH}_{3} \mathrm{PbI}_{3}$ and $\left.\mathrm{CH}_{3} \mathrm{NH}_{3} \mathrm{SnI}_{3}\right),{ }^{22,188}$ the vacancy-ordered perovskites do not show a similar trend. In $\mathrm{Cs}_{2} \mathrm{SnI}_{6}$, the hole effective masses are reasonably large, around $0.95 \mathrm{~m}_{0}$ for both directions. $\mathrm{Cs}_{2} \mathrm{TeI}_{6}$, again shows greater anisotropy, with hole effective masses of $0.65 \mathrm{~m}_{0}$ and $3.00 \mathrm{~m}_{0}$ seen for the $\Gamma \rightarrow \mathrm{X}$ and $\mathrm{W} \rightarrow$ $\mathrm{L}$ directions. In both cases, this can be attributed to a valence band dominated by non-bonding I $5 p$ states, resulting in limited dispersion.

\section{Reconciling small effective masses and ordered-vacancies}

The presence of dispersive conduction band states containing significant $S n 5 s$ contributions despite the existence of regular $S n$ vacancies, is, at first glance, puzzling. To investigate this behaviour, we have calculated charge density isosurfaces of the lowest conduction band of $\mathrm{Cs}_{2} \mathrm{SnI}_{6}$ (Fig. 6.9), at the $\Gamma$ and $\mathrm{X}$ points in the Brillouin zone. At the $\Gamma$ point, the $A_{1 g}$ antibonding orbital is in phase with the $A_{1 g}$ orbital in the adjacent cell (Fig. 6.9b), leading to a bonding interaction between neighbouring octahedra (Fig. 6.9c). This interaction occurs through significant portions of empty space, due to the disperse projection of the iodine $5 p$ orbitals into the interoctahedral void and acts to stabilise the bonding at this k-point. In contrast, at the X point, the $A_{1 g}$ antibonding orbital is out of phase with the $A_{1 g}$ orbital in the adjacent cell (Fig. 6.9d), resulting in an antibonding interaction between neighbouring octahedra (Fig. 6.9e). As such, the energy of the inter-
TABLE 6.2: Electron $\left(m_{\mathrm{e}}^{*}\right)$ and hole $\left(m_{\mathrm{h}}^{*}\right)$ effective masses of $\mathrm{Cs}_{2} \mathrm{Snl}_{6}$ and $\mathrm{Cs}_{2} \mathrm{Tel}_{6}$, calculated using HSE06+SOC. Masses given in units of electron rest mass, $m_{0}$ 
a)

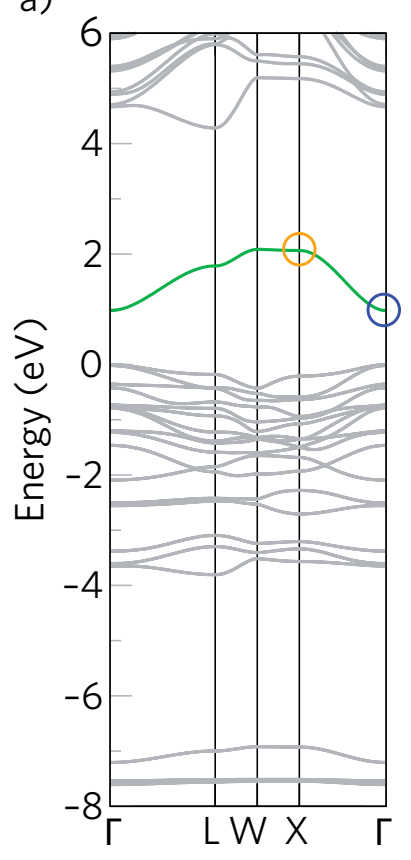

b)

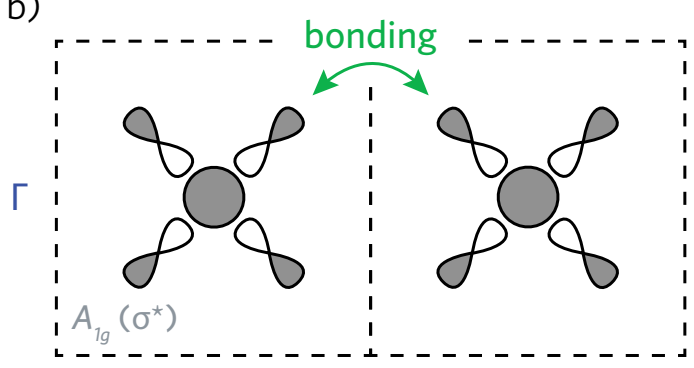

d)

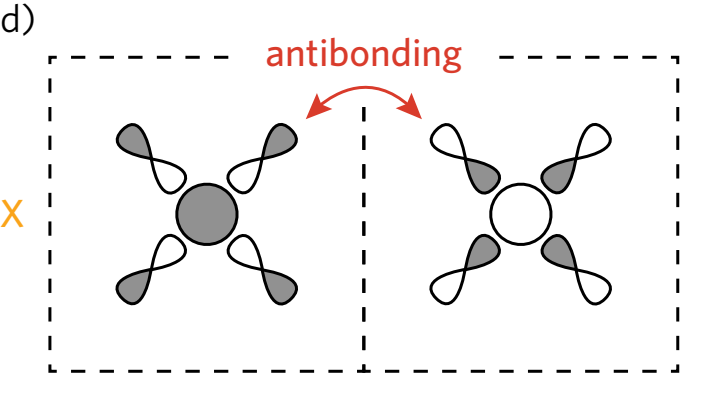

c)

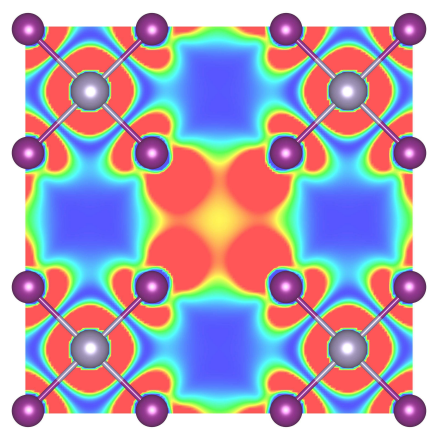

e)

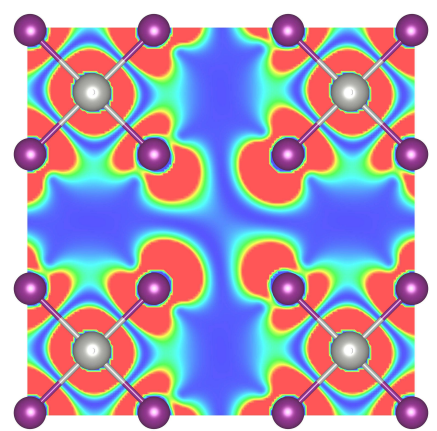

FIGURE 6.9: a) Band structure of $\mathrm{Cs}_{2} \mathrm{Snl}_{6}$, highlighting the $\mathrm{A}_{1 \mathrm{~g}}$ antibonding states. Simplified bonding diagram ( $a$ and $d$ ) and charge density isosurfaces (c and e) of the highlighted band at the $\Gamma$ and $X$ points. The isosurface level was set to $0.008 \mathrm{eV} \AA^{-3}$. action at the $\mathrm{X}$ point is significantly higher, giving rise to reasonably high band dispersion.

Interestingly, the bonding counterpart of the $A_{1 g}$ orbital (highlighted in 6.10a) shows significantly less dispersion. Again, this can be understood through charge density isosurfaces of the band at the $\Gamma$ and $X$ points. At $\Gamma$, there is a similar bonding interaction between octahedra in neighbouring cells (Fig. 6.10b), however, there is significantly less I $5 p$ projection into the void region (Fig. 6.10c). The interaction at $\mathrm{X}$ is again more antibonding (Fig. 6.10d), however, due to the reduced I $p$ contribution in the interoctahedral void (Fig. 6.10e), the destabilisation effect is dramatically reduced. Both effects combine to produce a band with limited dispersion across $\mathbf{k}$-space. The reduced I $p$ projection into the void region can be attributed to several factors: primarily, the bonding $A_{1 g}$ orbital contains less I $p$ character than the antibonding state, resulting in less overall charge density present on the iodine atoms. Furthermore, the bonding $A_{1 g}$ molecular orbital intrinsically shows reduced electron density projecting outside the octahedra when compared to the $A_{1 g}$ antibonding orbital, as depicted in Figures 6.10f and g. 
a)
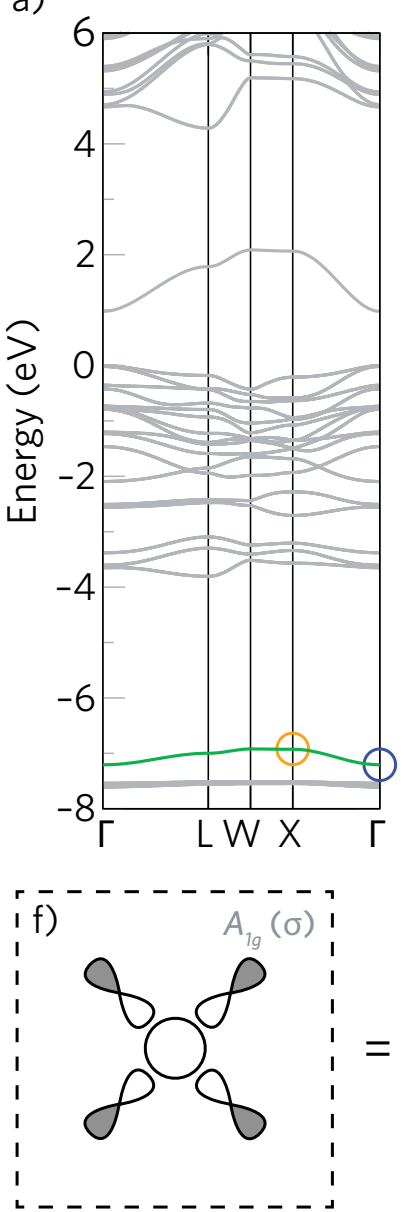

b)

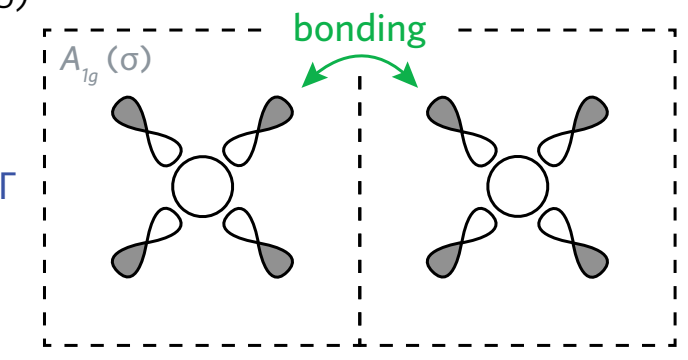

d)

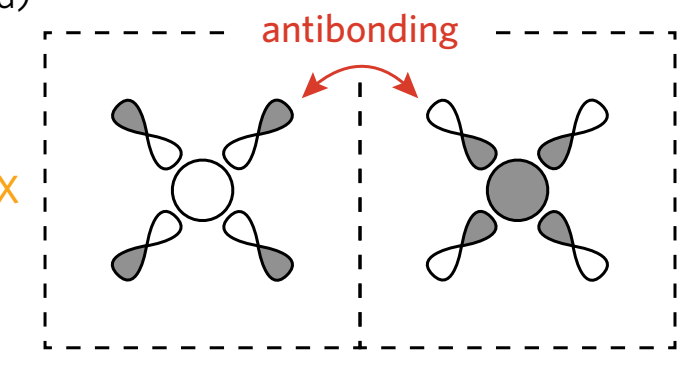

c)

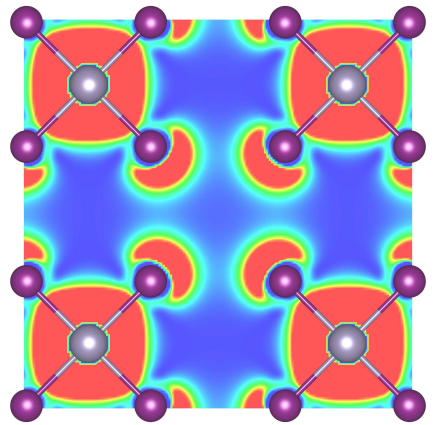

e)
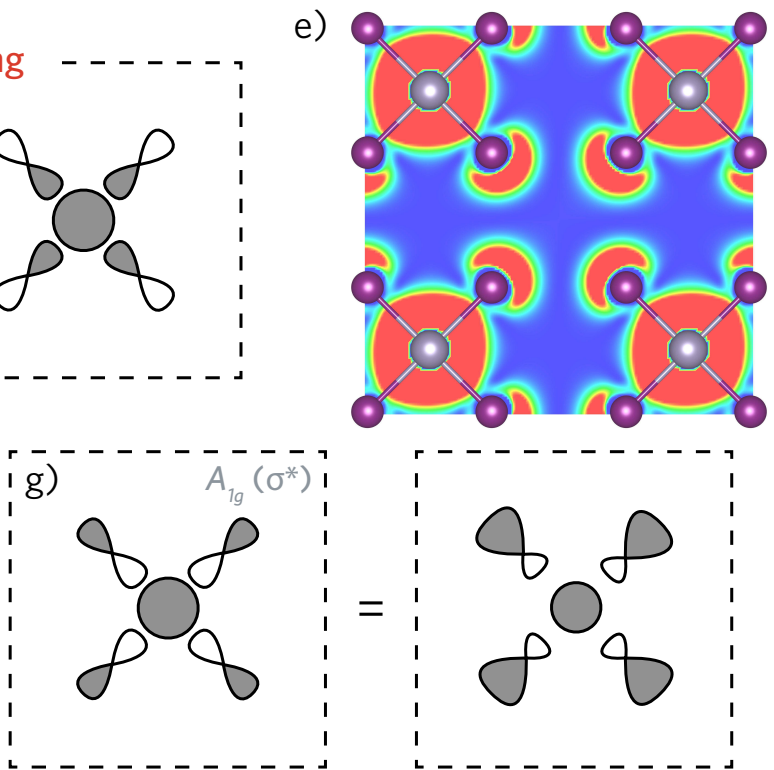

\section{Band alignments}

The band alignments of $\mathrm{Cs}_{2} \mathrm{SnI}_{6}$ and $\mathrm{Cs}_{2} \mathrm{TeI}_{6}$ relative to the vacuum level, calculated using HSE06 with a correction for spin-orbit coupling, are shown in Figure 6.11. As predicted based on the simplified molecular orbital picture presented in Figure 6.7, the valence band maximum, dominated by nonbonding I $5 p$ orbitals, is effectively pinned in energy across both compounds. The calculated ionisation potential of $\sim 5.80 \mathrm{eV}$ is in good agreement with the experimental value of $\sim 5.92 \mathrm{eV}$ for $\mathrm{Cs}_{2} \mathrm{SnI}_{6}$ determined by X-ray photoelectron spectroscopy. ${ }^{17}$ The ionisation potential of $\mathrm{Cs}_{2} \mathrm{SnI}_{6}$ is greater than that of its three-dimensional counter part, $\mathrm{CsSnI}_{3}(5.74 \mathrm{eV}),{ }^{252}$ as expected due to the population of the antibonding $A_{1 g}$ states at the top of the valence band. Due to the fixed ionisation potential, the electron affinity is directly controlled by the energy of the con-
FIGURE 6.10: a) Band structure of $\mathrm{Cs}_{2} \mathrm{Snl}_{6}$, highlighting the $A_{1 g}$ bonding states. Simplified bonding diagram ( $a$ and $d$ ) and charge density isosurfaces (c and e) of the highlighted band at the $\Gamma$ and $X$ points. The isosurface level was set to $0.008 \mathrm{eV} \AA^{-3}$. Schematic of electron distribution in a $f$ ) bonding and g) antibonding $A_{1 g}$ orbital. 


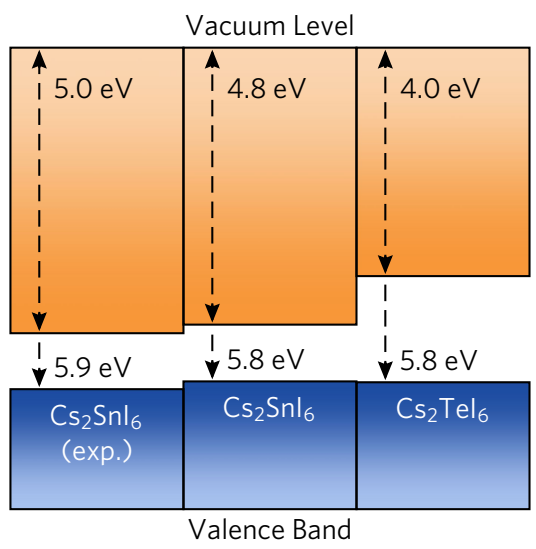

FIGURE 6.11: Experimental (exp.) and calculated (HSEO6+SOC) band alignment of $\mathrm{Cs}_{2} \mathrm{Snl}_{6}$ and $\mathrm{Cs}_{2} \mathrm{Tel}_{6}$ relative to the vacuum level. The experimental ionisation potential of $\mathrm{Cs}_{2} \mathrm{Snl}_{6}$ was determined by $\mathrm{X}$-ray photoelectron spectroscopy, with the electron affinity calculated based on the HSEO6+SOC band gap. duction band minimum. In $\mathrm{Cs}_{2} \mathrm{SnI}_{6}$ and $\mathrm{Cs}_{2} \mathrm{TeI}_{6}$, the antibonding Sn $s-\mathrm{I} p$ and antibonding Te $p-\mathrm{I} p$ states give rise to electron affinities of $4.8 \mathrm{eV}$ and $4.0 \mathrm{eV}$, respectively.

\section{Defect chemistry}

The experimental observation of a dramatic reduction in conductivity upon tellurium substitution requires an investigation into the defect properties of $\mathrm{Cs}_{2} \mathrm{SnI}_{6}$ and $\mathrm{Cs}_{2} \mathrm{TeI}_{6}$. A plot of the experimentally accessible chemical potentials for both compounds across a $\left(\mu_{\mathrm{Cs}}, \mu_{\mathrm{Te}}\right)$ plane ${ }^{262,263}$ is provided in Figure 6.12. The stability field is limited by the host conditions $\left(2 \mu_{\mathrm{Cs}}+\mu_{\mathrm{Sn}}=\Delta_{\mathrm{f}} H^{\mathrm{Cs}_{2} \mathrm{SnI}_{6}}\right.$ and $2 \mu_{\mathrm{Cs}}+\mu_{\mathrm{Te}}=$ $\Delta_{\mathrm{f}} H^{\mathrm{Cs}_{2} \mathrm{TeI}_{6}}$ ) to give the limits of Cs/Sn rich, Cs poor, and $\mathrm{Sn}$ poor environments for $\mathrm{Cs}_{2} \mathrm{SnI}_{6}$, with analogous environments for $\mathrm{Cs}_{2} \mathrm{TeI}_{6}$. Taking into account the limits imposed by the competing binary and ternary phases results in the stable regions shaded in orange. The accessible range of chemical potential space is significantly larger for $\mathrm{Cs}_{2} \mathrm{TeI}_{6}$, suggesting that formation of $\mathrm{Cs}_{2} \mathrm{SnI}_{6}$ will require greater sensitivity to the stoichiometric quantities of starting materials. Two potential environments have been highlighted, termed $A$ and $B$, which correspond to Cs/Sn-rich, I-poor (A) and Cs/Sn-poor, I-rich (B) conditions. For both compounds, we have explicitly considered the formation energies of the defects using the chemical potentials at the $\mathrm{A}$ point, as a representative middle ground. We note, however, that the B point is expected to most favour the formation of $n$-type $V_{\mathrm{I}}$ defects.

Previous research into $\mathrm{CsSnI}_{3}$, has identified iodine vacancies $\left(V_{\mathrm{I}}\right)$ as low energy shallow defects that are likely to impact conductivity. ${ }^{264}$ As such, to investigate the mechanism of conductivity in $\mathrm{Cs}_{2} \mathrm{SnI}_{6}$ and $\mathrm{Cs}_{2} \mathrm{TeI}_{6}$, we have considered the formation of donor $V_{\mathrm{I}}$ defects in both compounds. A plot of formation energy as function of Fermi level, under the chemical potentials at environment $\mathrm{A}$, is provided in Figure 6.13. For $\mathrm{Cs}_{2} \mathrm{SnI}_{6}$, the range of formation energies across all chemical potential environments is small $(0.14-0.39 \mathrm{eV})$, indicating that these defects are likely to be present in high concentrations. Furthermore, $V_{\text {I }}$ posses a relatively shallow $+1 / 0$ transition level $0.07 \mathrm{eV}$ 

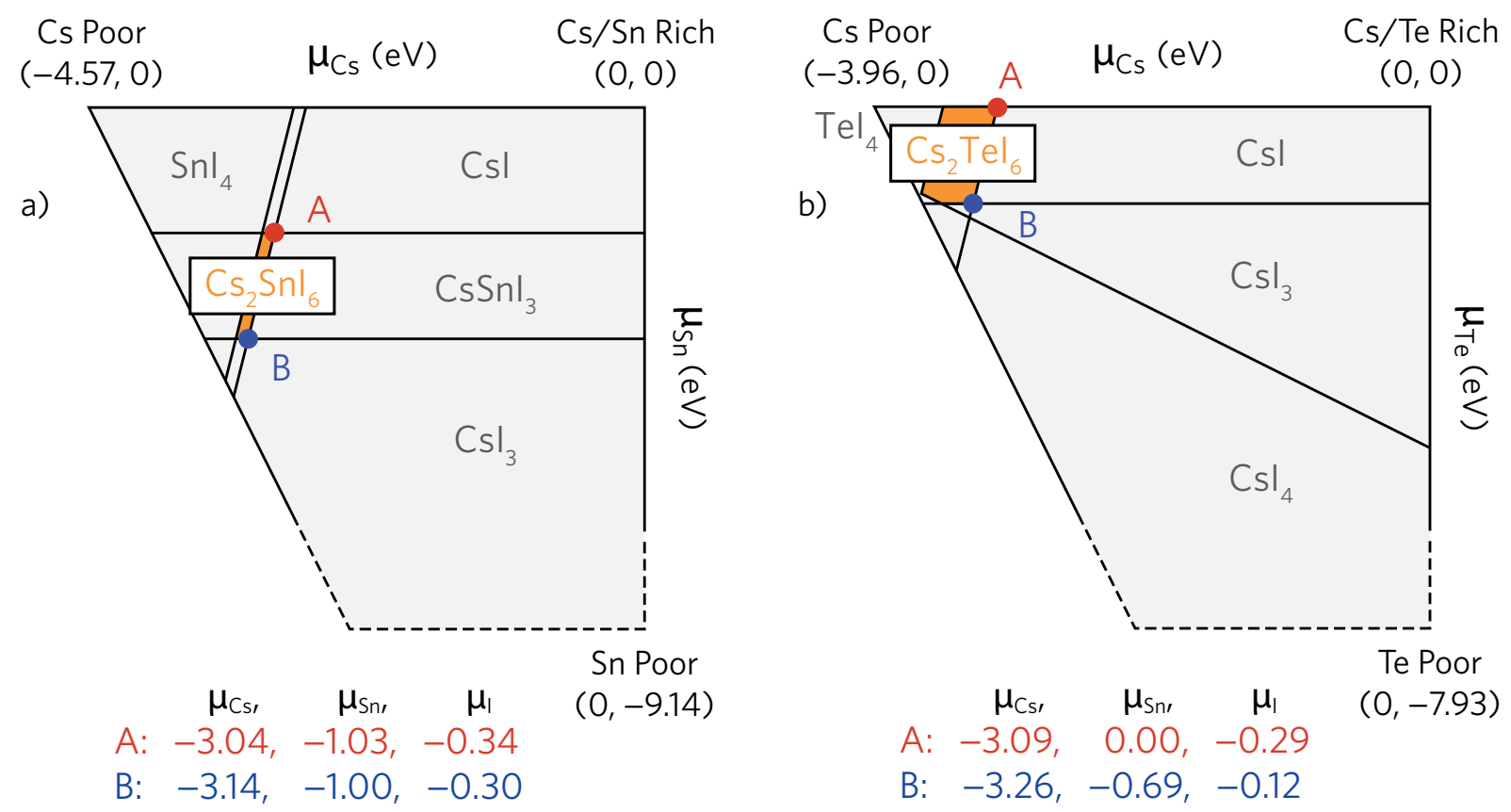

below the conduction band minimum, suggesting a source for the native $n$-type conductivity seen in experiment. Previous calculations have identified $V_{\mathrm{I}}$ as an ultra-deep donor, with a transition level $0.52 \mathrm{eV}$ beneath the conduction band minimum. ${ }^{244}$ Due the larger supercell size used in our calculations (232 versus 72 atoms), ${ }^{244}$ in addition to our correct treatment of the fundamental band gap - specifically, we do not erroneously adjust the amount of HF exchange to fit to the optical band gap - we believe our calculations are likely a better representation of the true defect chemistry. Indeed, our calculated transition level is more in keeping with a system displaying native $n$-type conductivity, with carrier concentrations up to $\sim 10^{17}$, as observed by our collaborators at Colorado State University. ${ }^{17}$

Moving to $\mathrm{Cs}_{2} \mathrm{TeI}_{6}$, the formation energy of $V_{\mathrm{I}}$, across all chemical potential environments, is considerably larger $(0.56-0.84 \mathrm{eV})$, indicating there will be fewer iodine vacancy defects present. Additionally, the $+1 / 0$ transition state is found ultra-deep within the band gap ( $0.63 \mathrm{eV}$ below the conduction band minimum). As such, $V_{\mathrm{I}}$ defects in $\mathrm{Cs}_{2} \mathrm{TeI}_{6}$ cannot produce samples with high $n$-type carrier concentrations. Interestingly, in both $\mathrm{Cs}_{2} \mathrm{SnI}_{6}$ and $\mathrm{Cs}_{2} \mathrm{TeI}_{6}$, the $V_{\mathrm{I}}+1 / 0$ defect level appears to be fixed in energy, around $0.9 \mathrm{eV}$ above the valence band maximum. These results suggest a possible source of the insu-

FIGURE 6.12: Illustration of the accessible chemical potential ranges of a) $\mathrm{Cs}_{2} \mathrm{Snl}_{6}$ and $b$ ) $\mathrm{Cs}_{2} \mathrm{Tel}_{6}$. Constraints imposed by the formation of competing binary and ternary compounds result in the stable region indicated in orange. 


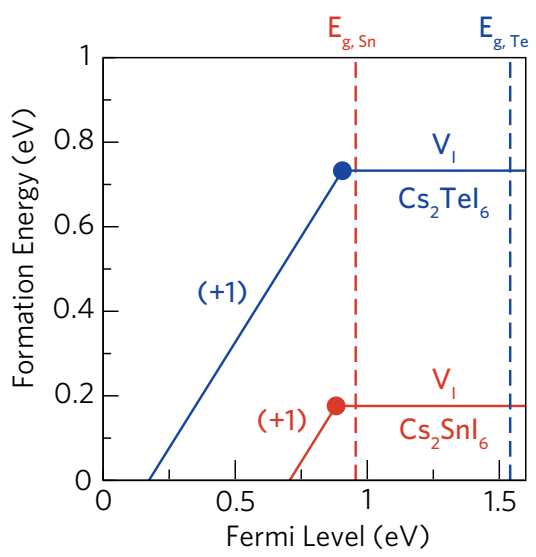

FIGURE 6.13: HSE06+SOC calculated defect formation energies for iodine vacancies in $\mathrm{Cs}_{2} \mathrm{Snl}_{6}$ (red) and $\mathrm{Cs}_{2} \mathrm{Tel}_{6}$ (green), under the chemical potential environment $A$, identified in Figure 6.12. Sloped lines indicate the +1 charge state and the solid dots represent the $+1 / 0$ transition levels. Dashed lines indicate the fundamental band gap of each material. lating behaviour observed in the solid-solution series, $\mathrm{Cs}_{2} \mathrm{Sn}_{1-x} \mathrm{Te}_{x} \mathrm{I}_{6}$ (Fig. 6.3). Namely, due to the pinned energy of the valence band maximum, the depth of the $V_{\mathrm{I}}+1 / 0$ transition level will depend solely on the electron affinity of the system. Accordingly, as the system becomes more Te rich, the conduction band minimum will rise in energy, resulting in a reduction in $n$-type carrier concentrations and lower levels of conductivity.

\section{Conclusions}

In this chapter, we have investigated the fundamental optoelectronic properties of the vacancy-ordered defect perovskites $\mathrm{Cs}_{2} \mathrm{SnI}_{6}$ and $\mathrm{Cs}_{2} \mathrm{TeI}_{6}$, in collaboration with our experimental partners Maughan et al. Using first-principles relativistic electronic structure theory, we show that $\mathrm{Cs}_{2} \mathrm{SnI}_{6}$ and $\mathrm{Cs}_{2} \mathrm{TeI}_{6}$ possess direct and indirect fundamental band gaps, respectively. Furthermore, we reveal that the fundamental band gap of $\mathrm{Cs}_{2} \mathrm{SnI}_{6}$ is symmetry disallowed, thereby providing an explanation for the apparent mismatch between theory and experiment previously discussed in the literature. The observation of dispersive conduction band states containing significant $\mathrm{Sn}$ contributions, despite the presence of regular vacancies, is attributed to inter-octahedral I-I interactions than span the voids in the lattice. We further reveal that these interaction are facilitated by the shape of the Sn 5s-I 5p $A_{1 g}$ antibonding orbital, which shows greater electron density projecting outside the octahedra. The trend in effective masses is insufficient to account for the dramatic reduction in carrier concentrations and electron mobilities seen upon tellurium substitution for the solid-solution series $\mathrm{Cs}_{2} \mathrm{Sn}_{1-x} \mathrm{Te}_{x} \mathrm{I}_{6}$. Instead, we attribute this behaviour to the depth of iodine vacancy defects, which are shallowest for $\mathrm{Cs}_{2} \mathrm{SnI}_{6}$ and become deeper with increasing tellurium incorporation. These results provide an explanation for the puzzling experimental observables detailed by our experimental collaborators. 


\section{Notes}

The work discussed in this chapter was featured in one publication:

1. A. E. Maughan, A. M. Ganose, M. M. Bordelon, E. M. Miller, D. O. Scanlon, and J. R. Neilson, Defect Tolerance to Intolerance in the Vacancy Ordered Double Perovskite Semiconductors $\mathrm{Cs}_{2} \mathrm{SnI}_{6}$ and $\mathrm{Cs}_{2} \mathrm{TeI}_{6}$, Journal of the American Chemical Society, 138, 8453-8464 (2016)

The optimised crystal structures, for all compounds discussed in this chapter, are provided in an online repository. ${ }^{251}$ 



\section{Part III}

\section{Bismuth-based absorbers}





\section{REVIEW: BISMUTH - B ASED PHOTOVOLTAICS}

Bismuth-based solar absorbers have recently attracted attention as a result of the similarities between the bismuth halides and lead hybrid perovskites. ${ }^{9,265,266}$ Both bismuth and lead adopt oxidation states two lower than their group valence, resulting in a stable $d^{10} s^{2} p^{0}$ electronic configuration. ${ }^{228}$ Furthermore, the $\mathrm{Bi}^{3+}$ and $\mathrm{Pb}^{2+}$ ions form a large variety of compounds with rich structural diversity. ${ }^{168,266-269}$ Bismuth also experiences large relativistic effects that act to beneficially increase the conduction band width and stabilise the material with respect to oxidation. ${ }^{164,188,270}$ Bismuth further presents an advantage over lead in that it is non-toxic and non-bioaccumulating, limiting the potential environmental impact if device encapsulation is compromised. ${ }^{271,272}$ The recent rise of bismuth in "green" catalysis and synthesis ${ }^{273,274}$ has prompted some to question whether bismuth absorbers can achieve similar success in photovoltaics. ${ }^{275}$

\section{Bismuth sulfide}

Bismuth sulfide $\left(\mathrm{Bi}_{2} \mathrm{~S}_{3}\right)$ possesses an optical band gap between 1.3$1.6 \mathrm{eV},{ }^{276-278}$ strong optical absorption, and low toxicity, making it an attractive candidate for photovoltaics (Fig. 7.1). While bismuth sulfide was originally tested for use in photoelectrochemical cells in the 1980 s, ${ }^{279,280}$ it was not until recently that $\mathrm{Bi}_{2} \mathrm{~S}_{3}$ was employed as a solar absorber. In particular, chemically deposited thin films of $\mathrm{Bi}_{2} \mathrm{~S}_{3}$ were combined with lead chalcogenides, enabling devices with efficiencies of $0.5 \%$ in $2011^{276}$ and $2.5 \%$ in $2013 .{ }^{281} \mathrm{As}^{\mathrm{Bi}_{2} \mathrm{~S}_{3} \text { pos- }}$ 


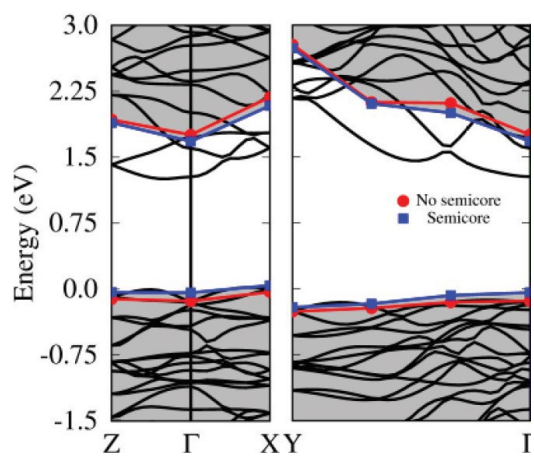

FIGURE 7.1: Band structure of $\mathrm{Bi}_{2} \mathrm{~S}_{3}$ calculated using HSEO6 with spin-orbit coupling. Taken from ref. 282.

a)

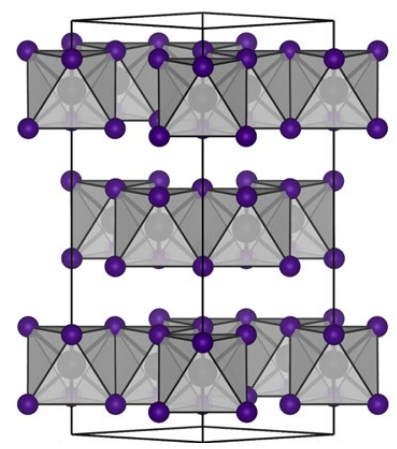

b)

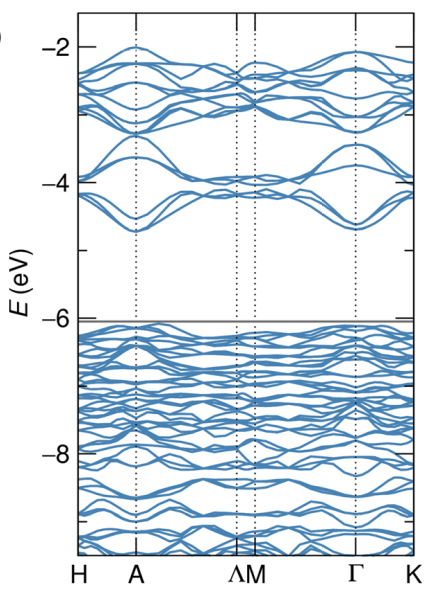

FIGURE 7.2: (a) Crystal structure and (b) band structure of $\mathrm{Bil}_{3}$, calculated using HSEO6 with spinorbit coupling. Bismuth and iodine atoms shown by grey and purple spheres, respectively. sesses intrinsic $n$-type conductivity, ${ }^{282}$ it has also been successfully employed in heterojunctions with $p$-type materials such as $\mathrm{PbS}$ quantum dots. ${ }^{283,284}$ These devices have achieved almost $5 \%$ power conversion efficiencies based on a "bulk-nano" heterojunction architecture. More recently, hybrid devices containing $\mathrm{Bi}_{2} \mathrm{~S}_{3}$ along with the organic absorber $\mathrm{P} 3 \mathrm{HT}$ have shown promising improvements in their efficiencies, reaching $3.3 \%$ in $2015 .{ }^{285-287}$ DFT calculations on the defect properties of $\mathrm{Bi}_{2} \mathrm{~S}_{3}$, however, suggest that performance may be limited due to the presence of deep trap states. ${ }^{288}$

\section{Bismuth iodide}

Bismuth iodide was recently identified - along with bismuth sulfide - as a potential solar absorber due to its suitable band gap and the possibility of high defect tolerance. ${ }^{9} \mathrm{BiI}_{3}$ crystallises in a layered rhombohedral crystal structure ${ }^{289}$ with a slightly indirect band gap of $1.67 \mathrm{eV}$ (Fig. 7.2). ${ }^{290,291}$ Initial devices produced by Lehner et al. obtained efficiencies of $0.3 \%$ with the $V_{\text {oc }}$ limited by poor alignment with the electron and hole contact layers. ${ }^{265,292}$ Subsequent work by Brandt et al. found that $\mathrm{BiI}_{3}$ possessed strong optical absorption, despite the indirect band gap, but short carrier lifetimes of 180-240 ps indicating high carrier recombination. ${ }^{266}$ In 2017, Hamdeh et al. reported devices with efficiencies up to $1.0 \%$, achieved through a solvent annealing process that optimised grain size and orientation. ${ }^{293}$ More recent DFT work investigating the defect properties of $\mathrm{BiI}_{3}$ reveals that all dominant point defects possess deep-level traps that can act as non-radiative recombination centres. ${ }^{294}$ As such, it will be difficult for $\mathrm{BiI}_{3}$ to match the performance of other emerging absorbers.

\section{Silver bismuth sulfide and iodide}

Nanocrystalline silver bismuth sulfide has recently gained attention as a photovoltaic absorber. Crystallising in a three-dimensional cu- 
bic distorted rocksalt structure, ${ }^{295,296}$ bulk $\mathrm{AgBiS}_{2}$ possesses an optical band gap of $0.9 \mathrm{eV} .{ }^{297}$ Strong quantum confinement effects, however, allow for quantum dot thin-films with band gaps ranging from $1.0-1.3 \mathrm{eV}$, depending on the size of crystallites, with strong optical absorption above $10^{5} \mathrm{~cm}^{-1}$. ${ }^{297-299}$ Initial devices containing $\mathrm{AgBiS}_{2}$ quantum dots as a sensitiser for $\mathrm{TiO}_{2}$ demonstrated poor efficiencies of less than a percent due to poor fill factors and short-circuit current densities. ${ }^{296,300}$ More recently, devices by Bernechea et al. containing tetramethylammonium iodide-treated $\mathrm{AgBiS}_{2}$ nanocrystals in a $p-i-$ $n$ solar cell architecture obtained record efficiencies of $6.3 \% .{ }^{301}$ This performance was enabled by a combination of high short-circuit current $\left(22 \mathrm{~mA} \mathrm{~cm}^{-2}\right)$ and fill factor (63\%), making $\mathrm{AgBiS}_{2}$ competitive with the best bismuth and antimony solar absorbers. Performance was limited by a relatively small open-circuit voltage $(0.45 \mathrm{eV})$ due to high levels of trap-assisted recombination. Accordingly, improvements in thin film synthesis is expected to further improve efficiencies.

$\mathrm{AgBi}_{2} \mathrm{I}_{7}$ is an emerging absorber that has only been sparsely characterised since its discovery in 1979. ${ }^{302}$ Based on an investigation into the $\mathrm{AgI}-\mathrm{BiI}_{3}$ solid solution, Kim et al. reported a direct band gap of $1.86 \mathrm{eV}$ for a crystal thought to be $\mathrm{AgBi}_{2} \mathrm{I}_{7}{ }^{303}$ A subsequent investigation using a combined theoretical and experimental approach indicated the band gap and X-ray diffraction data was instead a significantly better match for an $\mathrm{Ag}$-deficient $\mathrm{AgBiI}_{4}$ type structure. ${ }^{304}$ Regardless, when employed in a device with mesoporous $\mathrm{TiO}_{2}$ as the electron contact layer and P3HT as the hole contact layer, an efficiency of $1.12 \%$ was obtained. While the device showed a high fill factor of $67 \%$, its performance was limited by relatively low shortcircuit current and open-circuit voltage. In 2017, Turkevych et al. ${ }^{305}$ examined a series of $\mathrm{Ag}_{a} \mathrm{Bi}_{b} \mathrm{I}_{x}$ (where $x=a+3 b$ ) Rudorffites structures, including $\mathrm{Ag}_{3} \mathrm{BiI}_{6}, \mathrm{Ag}_{2} \mathrm{BiI}_{5}, \mathrm{AgBiI}_{4}$, and $\mathrm{AgBi}_{2} \mathrm{I}_{7}$. Despite all compounds possessing relatively large band gaps in the range 1.79$1.83 \mathrm{eV}$, their champion device containing $\mathrm{Ag}_{3} \mathrm{BiI}_{6}$ on mesoporous $\mathrm{TiO}_{2}$ achieved efficiencies of $4.3 \%$. While very promising for a proofof-concept device, similar to many bismuth-based photovoltaic ab- 
sorbers, additional attention will be required to achieve significant performance improvements in the further.

\section{Caesium and hybrid bismuth iodides}
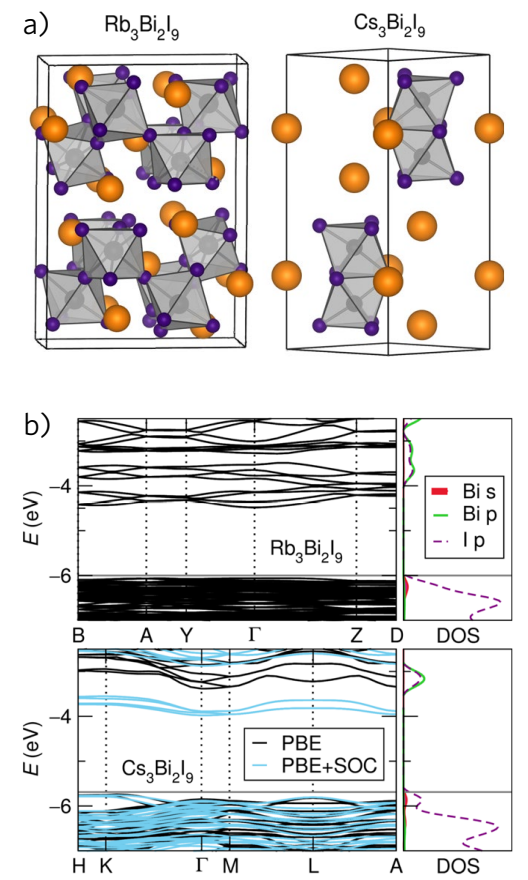

FIGURE 7.3: (a) Crystal structure and $(b)$ band structure of $\mathrm{Rb}_{3} \mathrm{Bi}_{2} \mathrm{l}_{9}$ and $\mathrm{Cs}_{3} \mathrm{Bi}_{2} \mathrm{I}_{9}$, calculated using HSEO6 with spin-orbit coupling. Bismuth and iodine atoms shown by grey and purple spheres, respectively.
Caesium bismuth iodide $\left(\mathrm{Cs}_{3} \mathrm{Bi}_{2} \mathrm{I}_{9}\right)$ and its methylammonium analogue $\left(\left(\mathrm{CH}_{3} \mathrm{NH}_{3}\right)_{3} \mathrm{Bi}_{2} \mathrm{I}_{9}\right)$ have attracted attention due to the focus on their antimony counterparts. ${ }^{306}$ At room temperature, both compounds crystallise in a "zero-dimensional" structure containing isolated face-sharing octahedral $\left[\mathrm{Bi}_{2} \mathrm{I}_{9}\right]^{3-}$ dimers (Fig. 7.3a). The $\mathrm{Cs}^{+}$ and $\left(\mathrm{CH}_{3} \mathrm{NH}_{3}\right)^{+}$cations are non-bonding and serve to charge balance the structure. ${ }^{307-309}$ Experimental work combined with density functional theory results, indicate an indirect band gap of $1.9 \mathrm{eV}$ with the low dimensionality producing localised electronic states (Fig. 7.3b), suggesting carrier mobilities will be low. ${ }^{265}$ The limited dispersion, however, gives rise to strong optical absorption, which may make these materials suitable for photovoltaics. ${ }^{310,311}$

Initial devices containing mesoporous $\mathrm{TiO}_{2}$ with $\mathrm{Cs}_{3} \mathrm{Bi}_{2} \mathrm{I}_{9}$ and $\left(\mathrm{CH}_{3} \mathrm{NH}_{3}\right)_{3} \mathrm{Bi}_{2} \mathrm{I}_{9}$ performed poorly, achieving efficiencies of $\sim 1 \%$ and $0.2 \%$, respectively. ${ }^{312}$ In both cases, performance was limited by small short-circuit current and fill factor, with the open-circuit voltage remaining fairly large $(\sim 0.85 \mathrm{eV})$. Subsequent studies on $\left(\mathrm{CH}_{3} \mathrm{NH}_{3}\right)_{3} \mathrm{Bi}_{2} \mathrm{I}_{9}$ employing alternative hole contact layers showed small improvements in $J_{\mathrm{sc}}{ }^{313}$ and fill factor, ${ }^{314}$ but again suffered from low efficiencies. The photoluminescence behaviour of $\left(\mathrm{CH}_{3} \mathrm{NH}_{3}\right)_{3} \mathrm{Bi}_{2} \mathrm{I}_{9}$ indicated a charge-carrier recombination lifetime of $760 \mathrm{ps}$, a factor of $10^{3}$ lower than those found in the best MAPI films. ${ }^{310}$ Based on a low photoluminescence quantum efficiency of $0.4 \%$ and the observation of defect states through X-ray photoelectron spectroscopy, non-radiative recombination pathways are thought to be the source of the small carrier lifetimes. ${ }^{312}$ In 2018, use of vapour-assisted solution process deposition enabled $\left(\mathrm{CH}_{3} \mathrm{NH}_{3}\right)_{3} \mathrm{Bi}_{2} \mathrm{I}_{9}$ based devices with efficiencies up to $3.17 \%$. Impressively, the devices showed very high open-circuit voltages up to $1.0 \mathrm{eV}$ and less than $0.1 \%$ loss in efficiency when exposed to air for 60 days. Such a high stability is a strong motivator 
for additional work on improving device efficiencies.

Another hybrid inorganic-organic bismuth iodide that is of interest as a solar absorber is the one-dimensional hexanediammonium bismuth iodide, $\left(\mathrm{H}_{3} \mathrm{NC}_{6} \mathrm{H}_{12} \mathrm{NH}_{3}\right) \mathrm{BiI}_{5}$. Possessing a relatively large band gap around $2 \mathrm{eV}$, it was recently employed in a device with mesoporous $\mathrm{TiO}_{2} \cdot{ }^{315}$ Despite excellent film coverage and thermal stability, the device performed poorly with a low open-circuit voltage and short-circuit of $0.38 \mathrm{eV}$ and $0.10 \mathrm{~mA} \mathrm{~cm}^{-2}$, respectively.

\section{Outlook}

The rise of the hybrid perovskites has stimulated research into leadfree alternatives. Bismuth-based materials are attractive due to their earth-abundant and non-toxic nature, combined with promising optoelectronic properties. Accordingly, the field of bismuth-based photovoltaics has blossomed over the last 3-4 years and is expected to continue to grow. Indeed, the best performing devices show comparable efficiencies to the best-in-class antimony- and tin-based alternatives. In particular, the three-dimensional cubic structure of $\mathrm{AgBiS}_{2}$ draws many parallels to the hybrid perovskites, and impressive efficiencies of over $6 \%$ highlight its potential as an emerging photovoltaic. Despite this, further work is clearly needed if bismuthbased materials are to achieve success in an already highly competitive solar panel market. 



\section{BISMUTHCHALCOIODIDES}

\section{Introduction}

In 2015, Brandt et al. identified two bismuth-based materials, BiSI and BiSeI, as potential solar absorbers. ${ }^{9}$ Both compounds are isostructural, crystallising in a 1D ribbon structure held together by weak dispersive forces (Fig. 8.1). ${ }^{268,316}$ As proposed by Zhou et al., this type of structure may have many advantages for solar applications. If the crystal is oriented so that the $1 \mathrm{D}$ ribbons are vertically aligned on the substrate (e.g. the hole or electron contact materials in a photovoltaic device), a direct pathway along the covalently bonded axis of the ribbons connects the device contacts, potentially allowing for efficient carrier extraction routes. Furthermore, if vertically aligned growth is enforced, any grain boundaries or disconnections within the crystal should not disrupt the conduction pathways and instead occur parallel to the ribbons, avoiding the formation of dangling bonds and associated defect trap sites. ${ }^{317}$ This is particularly important for solar cells, where defect sites are a primary cause of recombination losses. The advantages of this geometry have recently been demonstrated in the similarly structured compound, $\mathrm{Sb}_{2} \mathrm{Se}_{3}$, where devices containing vertically oriented films achieved over $2 \%$ greater efficiency than their non-oriented counterparts. ${ }^{317}$

The only functioning photovoltaic devices containing BiSI and BiSeI reported in the literature, were produced by Hahn et al. in 2012. ${ }^{318,319}$ Both compounds were found to possess large absorption coefficients and band gaps of $1.57 \mathrm{eV}$ and $1.29 \mathrm{eV}$, respectively, ideal for solar cell applications. Their devices - employing $p$-CuSCN as the hole transport layer and fluorine-doped tin oxide (FTO) as the transpar- 
a)

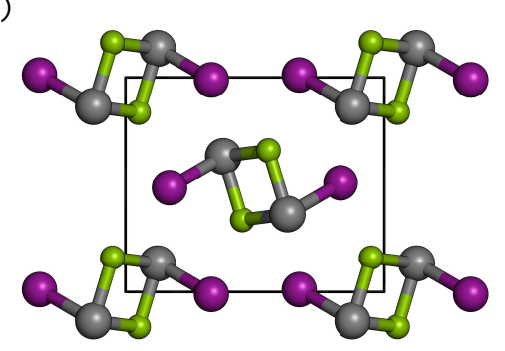

b)

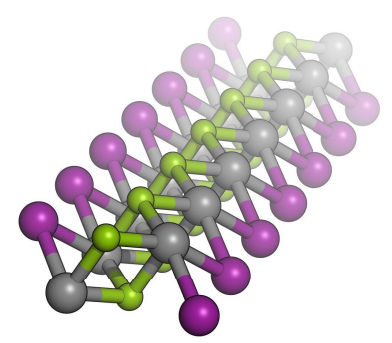

c)

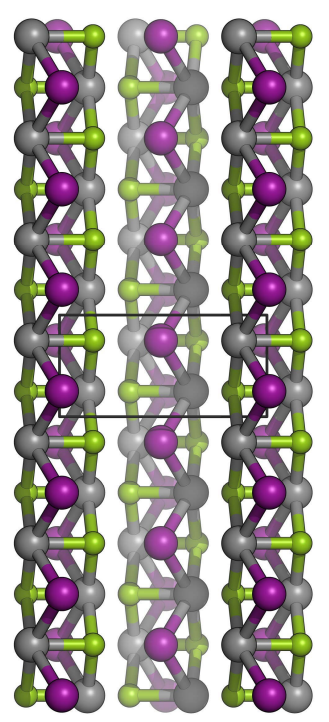

FIGURE 8.1: BiSI crystal structure viewed along (a) the [100] direction, (b) a perspective highlighting the 1D nature of the ribbons, and (c) the [001] direction. Se, Bi, and $\mathrm{I}$ atoms are denoted by green, grey, and purple spheres, respectively. ent contact - performed poorly, only achieving efficiencies up to $0.012 \%$. The authors attribute the unsatisfactory performance of their devices to small hole diffusion lengths of $50 \mathrm{~nm},{ }^{319}$ proposing that high levels of charge screening at the heterojunction interface prevented effective electron-hole separation and resulted in increased rates of recombination. This explanation is, however, at odds to other solar absorbers with large dielectric constants, such as MAPI where efficient charge screening is thought to provide increased tolerance to defects and reduce unwanted carrier recombination. 9,22,236 Instead, based on our previous discussion of the $1 \mathrm{D}$ ribbon structure, it is likely that the small hole diffusion lengths resulted from the formation of polycrystalline films, which disrupt the pathways for carrier extraction and increase recombination rates. More recently, advancements in low-temperature synthesis routes has enabled BiSI films with improved photon-to-current conversion efficiency (IPCE). ${ }^{320}$ As such, these materials possess many of the ideal properties required in efficient photovoltaics and, therefore, warrant a detailed analysis of their electronic properties.

\section{Methodology}

Calculations were performed using the Vienna $A b$ initio Simulation Package. A k-point mesh of $\Gamma$-centred $3 \times 6 \times 2$ and plane wave cutoff of $400 \mathrm{eV}$ was found to converge the 12 atom unit cells of BiSI and BiSeI to within $1 \mathrm{meV} /$ atom. During geometry optimisations, the cutoff was increased to $520 \mathrm{eV}$ to avoid errors resulting from $\mathrm{Pu}$ lay stress. ${ }^{85}$ The structures were deemed converged when the forces totalled less than $10 \mathrm{meV} \AA^{-1}$.

Several functionals were used in this work: for geometry relaxations, PBEsol and PBE were employed, with and without the addition of Grimme's D3 dispersion correction. Electronic properties were calculated using HSE06 with the addition of spin-orbit coupling effects (HSE06+SOC). The Brillouin zone for the Pnma space group, indicating the high-symmetry points explored in the band 
structure, is provided in Fig. 8.2. Density functional perturbation theory (DFPT) was employed, in combination with the PBEsol functional, to calculate the ionic contribution to the dielectric constants, with a denser $\mathbf{k}$-point mesh of $\Gamma$-centred $8 \times 14 \times 6$ required to achieve convergence. Projected crystal orbital Hamilton population (pCOHP) analysis was performed using the LOBSTER program, based on wavefunctions calculated using HSE06. ${ }^{92,95}$

For band alignment calculations, the core-level alignment approach of Wei and Zunger was employed, ${ }^{217}$ using a slab model with $20 \AA$ of vacuum and a $20 \AA$ thick slab. The slab was cleaved along the non-polar (110) surface, due to the absence of any dangling bonds. Due to the size of the model, which precluded the use of HSE06+SOC, band alignment calculations were performed using HSE06, with an explicit correction to the band gap and valence band maximum position taken from the HSE06+SOC calculated bulk.

Defect calculations were performed in a $2 \times 3 \times 1$ supercell containing 72 atoms, using the PBEsol functional and a $\Gamma$-centred $2 \times 3 \times 3$ k-point mesh. As PBEsol reproduces the experimental band gaps of both compounds, the defect transition levels will not need to be extrapolated to account for band gap underestimation. Despite this, as generalised-gradient approximation functionals can suffer from the self-interaction error, some unphysical delocalisation of defect states may occur. This may manifest as incorrect stabilisation of partially or unionised defects. The defect energies were corrected to account for use of a finite-sized supercell using the potential level alignment, band-filling, and image-charge corrections described in Chapter 3. The SC-FERMI code was utilised to calculate the self-consistent Fermi level and corresponding defect concentrations.

\section{Results}

\section{Geometric structure}

BiSI and BiSeI share the same crystal structure as SbSI (Pnma), whereby $1 \mathrm{D}$ ribbons of $\mathrm{BiChI}$ are held together by weak van der Waals type

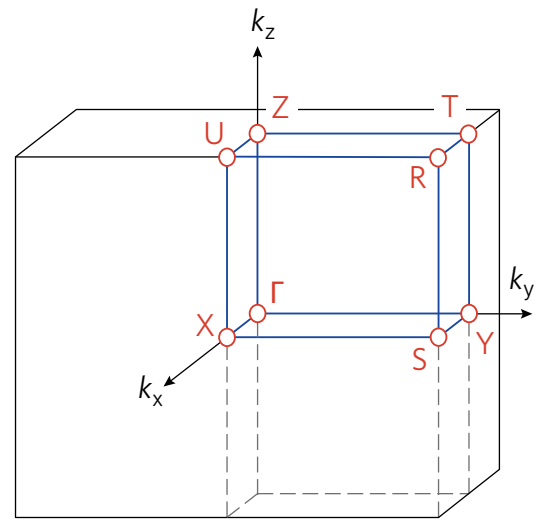

FIGURE 8.2: Brillouin zone of the Pnma space group. Coordinates of the high symmetry points used for the band structures and effective masses: $\Gamma=(0,0,0) ; Y$ $=(1 / 2,0,0) ; X=(0,1 / 2,0) ; Z=$ $(0,0,1 / 2) ; U=(0,1 / 2,1 / 2) ; T=$ $(1 / 2,0,1 / 2) ; S=(1 / 2,1 / 2,0) ; R=$ $(0,1 / 2,1 / 2)$. 
TABLE 8.1: Lattice parameters of $\mathrm{BiSI}$ and BiSel, with percentage difference from experiment 268,316 given in parentheses. Lattice parameters and bond lengths are given in $\AA$, with all cell angles found to be $90^{\circ}$. The optimised crystal structures are available online in a public repository 321 interactions (Fig. 8.1). The ribbons are formed of distorted edgesharing pseudo-octahedra, containing three $\mathrm{Bi}-\mathrm{Ch}$ and two $\mathrm{Bi}-\mathrm{I}$ bonds, with the lone pair on the Bi occupying a vacant site. ${ }^{268,316}$ The $\mathrm{Bi}-\mathrm{Ch}$ bonds can be categorised into those occurring parallel $\left(\mathrm{Bi}-\mathrm{Ch}_{\|}\right)$or perpendicular $\left(\mathrm{Bi}-\mathrm{Ch}_{\perp}\right)$ to the axis of the ribbons. Both structures were geometrical optimised using the PBE and PBEsol functionals, both with and without explicit treatment of dispersive interactions (Table 8.1). The PBEsol functional, in the absence of the D3 correction, was found to provide the most accurate description of lattice parameters and bond lengths, with all distances found to be within $1 \%$ of the experimental crystal structure. In both compounds the $a$ and $b$ lattice parameters were underestimated, indicating that thermal effects may play a role in dictating the distance between the ribbons.

\begin{tabular}{lccc}
\hline BiSI & $a(\AA)$ & $b(\AA)$ & $c(\AA)$ \\
\hline PBEsol & $8.44(-0.94 \%)$ & $4.13(-0.96 \%)$ & $10.26(0.79 \%)$ \\
PBEsol+D3 & $8.34(-2.11 \%)$ & $4.14(-0.72 \%)$ & $10.05(-1.28 \%)$ \\
PBE & $8.81(3.40 \%)$ & $4.20(0.72 \%)$ & $10.37(1.87 \%)$ \\
PBE+D3 & $8.66(1.64 \%)$ & $4.21(0.96 \%)$ & $11.21(10.12 \%)$ \\
\hline Experiment & 8.52 & 4.17 & 10.18 \\
\hline \multicolumn{4}{c}{$b(\AA)$} \\
\hline BiSeI & $a(\AA)$ & $c(\AA)$ \\
\hline PBEsol & $8.63(-0.80 \%)$ & $4.19(-0.71 \%)$ & $10.58(0.09 \%)$ \\
PBEsol+D3 & $8.53(-1.95 \%)$ & $4.19(-0.71 \%)$ & $10.35(-2.08 \%)$ \\
PBE & $8.88(2.07 \%)$ & $4.28(1.42 \%)$ & $10.69(1.14 \%)$ \\
PBE+D3 & $8.81(1.26 \%)$ & $4.25(0.71 \%)$ & $12.03(13.81 \%)$ \\
\hline Experiment & 8.70 & 4.22 & 10.57 \\
\hline
\end{tabular}

\section{Electronic properties}

Figures 8.3a and $\mathrm{c}$ show the band structures of BiSI and BiSeI, respectively, calculated using HSE06+SOC. The inclusion of spin-orbit coupling was tested and found to be essential to accurately describe the electronic structure of both materials (a comparison of the band structures with and without spin-orbit coupling can be found in Fig. C.1 of Appendix C). The relativistic renormalisation of the conduction 
a)

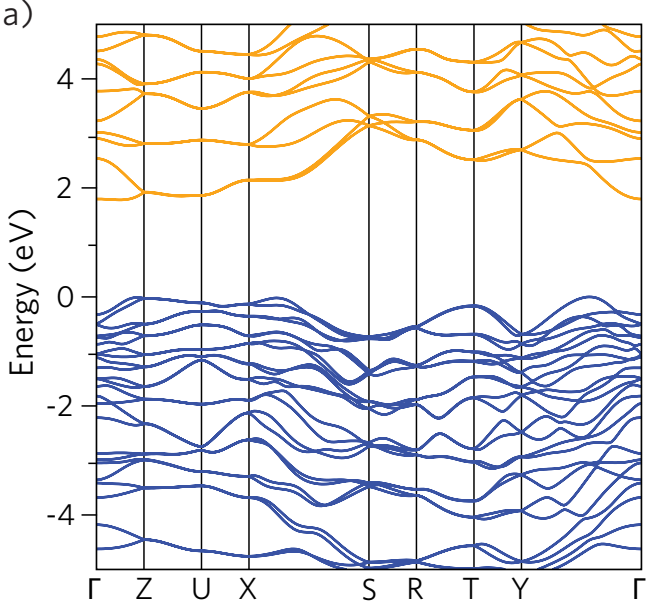

c)

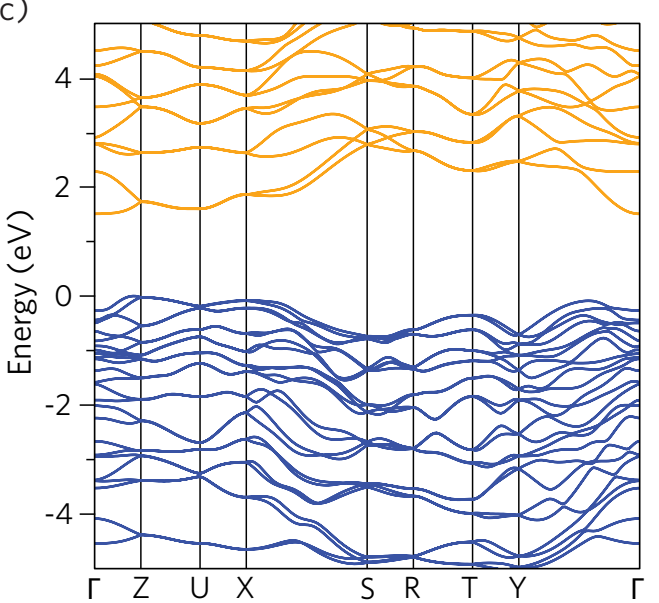

b)

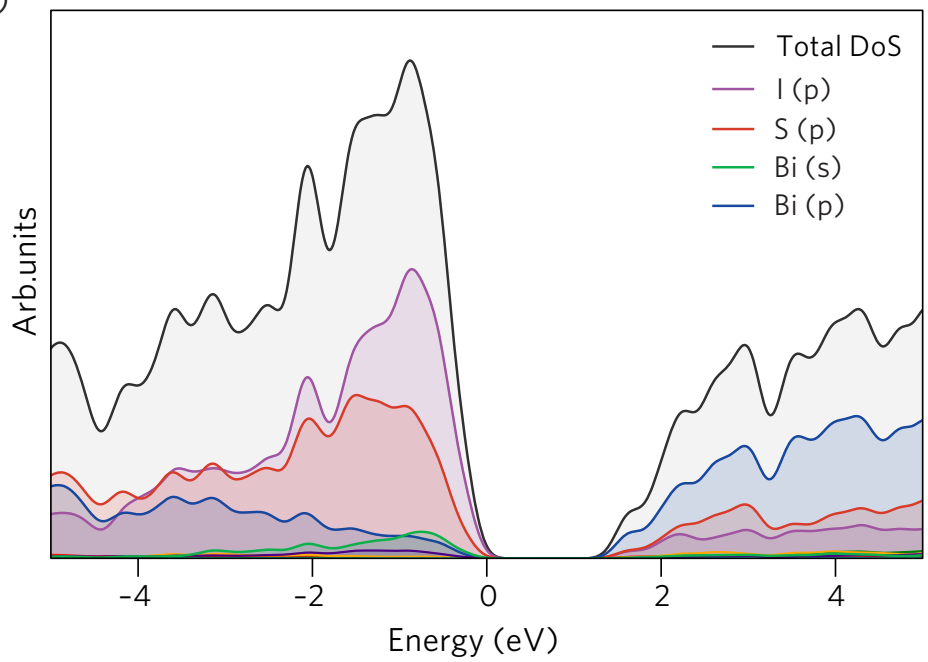

d)

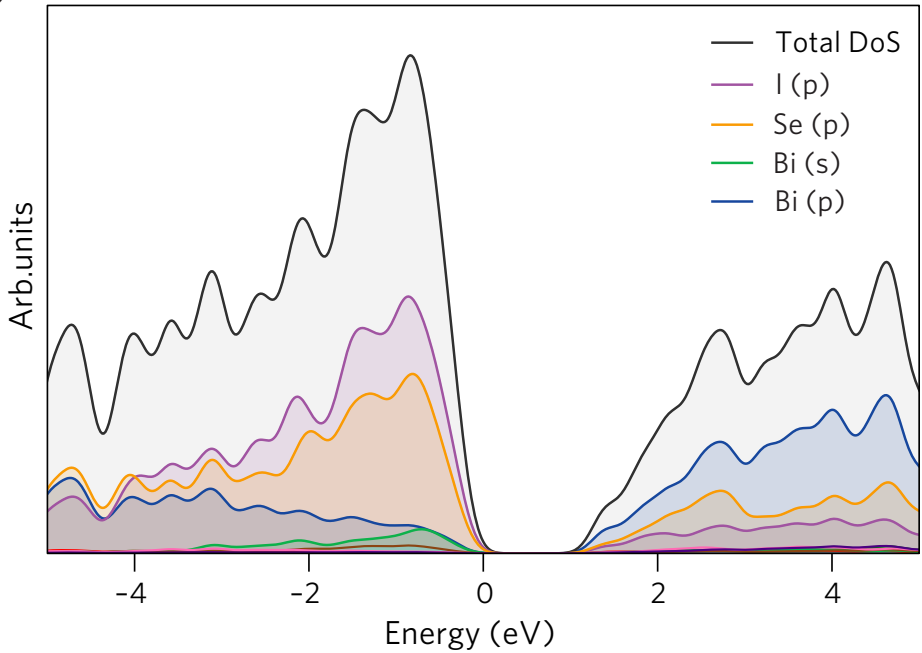

band was found to be over $0.6 \mathrm{eV}$ in both cases. Both band structures show broadly the same features, as expected as BiSI and BiSeI are isoelectronic and isostructural. A primary difference between the band structures is the position of the band edges; in BiSI the valence band maximum (VBM) and conduction band minimum (CBM) appear between $\mathrm{Y}-\Gamma$ and $\Gamma-\mathrm{Z}$, respectively. In contrast, the VBM of BiSeI is shifted to between $\Gamma-Z$, with the CBM situated on $\Gamma$. The HSE06+SOC calculated band gaps of BiSI and BiSeI were found to be $1.78 \mathrm{eV}$ and $1.50 \mathrm{eV}$, respectively. We note that the band gaps of both compounds are strongly dependent on temperature $\left(\mathrm{d} E_{\mathrm{g}} / \mathrm{d} T\right.$ $=-7.0 \times 10^{-4} \mathrm{eV} \mathrm{K}^{-1}$ for BiSI and $-6.5 \times 10^{-4} \mathrm{eV} \mathrm{K}^{-1}$ for BiSeI), ${ }^{322}$ in part due to the weak interactions between ribbons that allow for no-
FIGURE 8.3: (a, c) HSE06+SOC calculated band structure and ( $b$, d) partial density of states, for BiSI and BiSel, respectively. The valence band maximum has been shifted to zero $\mathrm{eV}$ in all cases. In panels (a) and (c), the colours blue and orange denote the valence and conduction bands, respectively. 
ticeable thermal expansion. As the HSE06+SOC results fail to account for thermal effects, we have calculated the room temperature $(293 \mathrm{~K})$ band gaps using the experimental corrections above. The resulting temperature corrected band gaps of $1.57 \mathrm{eV}$ (BiSI) and $1.31 \mathrm{eV}$ (BiSeI), are in excellent agreement with the room temperature experimental optical band gaps of 1.56-1.59 eV (BiSI) and 1.29-1.32 eV (BiSeI). ${ }^{322,323}$ As previously observed in the group 15 chalcohalides, ${ }^{324,325}$ the fundamental band gaps are both indirect $\left(E_{\mathrm{g}}^{\text {ind }}\right)$. However, the direct band gaps $\left(E_{\mathrm{g}}^{\mathrm{dir}}\right)$ - which are more relevant for thin film absorbers due to the need for strong optical absorption - are only slightly larger $(\sim 0.1 \mathrm{eV})$, indicating that these materials are still ideal for photovoltaic applications.

The ion decomposed density of states (DOS), calculated using HSE06+SOC, is provided in Figures $8.3 \mathrm{~b}$ and d. Both show similar features, with the VBM composed of mixing between the I and $\mathrm{Ch} p$ states with some Bi $s$ and $p$ states present, and the CBM dominated by $\mathrm{Bi} p$ states with the I and $\mathrm{Ch} p$ states contributing further into the conduction band. On moving from BiSI to BiSeI, the increase in energy of the Se $p$ states acts to push up the VBM, resulting in a smaller band gap. We note that this shift is in line with the shift in ionisation potential down the chalcogenide group. ${ }^{23}$

\section{The bismuth lone pair}

The appearance of the Bi $s$ and $p$ states toward the top of the valence band can be rationalised through analysis of the Bi lone pair. Similar to many of the heavier post-transition metals, bismuth adopts a valence state two lower than its group valence due to the stability of the $d^{10} s^{2} p^{0}$ electronic configuration. Traditionally, lone pair theory predicts that the formation of a stereochemically active lone pair (one that results in a distortion to the crystal structure) is the result of onsite hybridisation of non-bonding Bi $s$ and $p$ states at the VBM. ${ }^{326,327}$ However, in many lone pair systems, such as $\mathrm{PbS}^{328}$ and $\mathrm{CuBiCh}_{2},{ }^{329}$ the metal $s$ and $p$ states are too far apart in energy for hybridisation to occur ( $11 \mathrm{eV}$ difference in BiSI). Instead, as detailed in the revised lone pair model by Walsh and Watson, ${ }^{228}$ the metal $s$ states mix with 
anion $p$ states, resulting in occupied bonding and anti-bonding states at the top and bottom of the valence band, respectively. This can be seen in the pCOHP analysis provided in Figure 8.4. An electronic structure of this type, where the VBM is composed of anti-bonding states, can often result in significantly improved defect tolerance, as dangling bonds formed in the presence of vacancy defects are likely to appear resonant in the valence band. ${ }^{18,330}$

A stereochemically active lone pair will result if the anti-bonding states at the top of the valence band contain a substantial metal $s$ contribution. In this case, these states can mix with the unoccupied metal $p$ orbitals, resulting in a significant stabilisation effect due to the formation of an asymmetric electron density and distortion of the crystal structure. ${ }^{331-333}$ As this effect is dependent on the concentration of metal $s$ states at the top of the valence band and this in turn is dictated by the degree of mixing of the anion $p$ and metal $s$ states, the strength of the lone pair is primarily controlled by the relative energies of these states. Metal $s$ and anion $p$ states that are closer in energy will result in stronger lone pairs and, as such, the lone pair effect is seen to lessen when moving down the chalcogen group. When considering heavy metal atoms, such as $\mathrm{Bi}$, which experience strong relativistic effects, the metal $6 s$ states are significantly reduced in energy due the scalar contraction of the $s$-shell, and a further reduction in lone pair strength is observed. ${ }^{228,334}$ Accordingly, the $\mathrm{Bi}$ lone pairs are relatively diffuse in BiSI and BiSeI, as can be seen in the charge density isosurface of the VBM shown in Figure 8.5a. The distortion of the pseudo-octahedra can also be used as a quantitative measure of lone pair strength, and is given by the average deviation in bond angles from the idealised angle of $90^{\circ}\left(\sigma_{\text {oct }}\right)$. This distortion can be seen to decrease moving from BiSI $\left(\sigma_{\text {oct }}=7.49^{\circ}\right)$ to BiSeI $\left(\sigma_{\text {oct }}=6.61^{\circ}\right)$, as expected based on the relative strength of the lone pair. In comparison, materials with highly stereochemically active lone pairs, such as $\mathrm{Sb}_{2} \mathrm{O}_{3}$, possess significantly greater octahedral distortion $\left(\sigma_{\text {oct }}=21.82^{\circ}\right) .{ }^{335}$

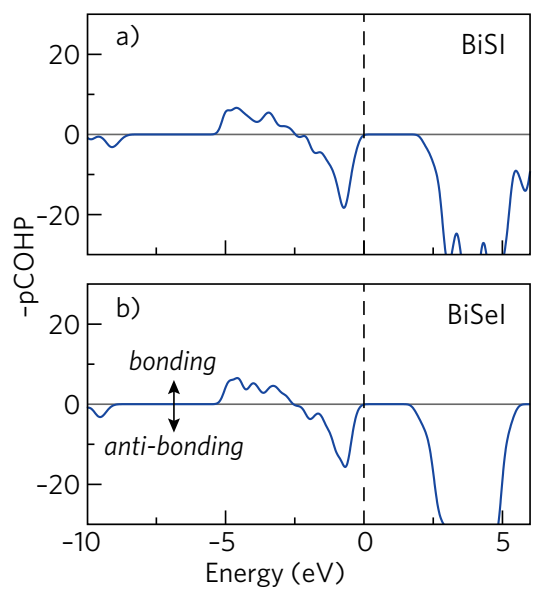

FIGURE 8.4: Projected crystal orbital Hamilton population (pCOHP) analysis of (a) BiSI and (b) BiSel, in which the density of states is partitioned with the sign denoting bonding or anti-bonding character, and the magnitude indicating the strength of the interaction. For each compound the averaged pCOHP across all pairwise interactions in the unit cell is presented. The valence band maximum is set to zero $\mathrm{eV}$. 
FIGURE 8.5: Charge density isosurfaces of (a) the valence band maximum and (b) the conduction band minimum of BiSel. The colours blue and red indicate areas of low and high electron density, respectively. $\mathrm{Bi}, \mathrm{Se}$, and I atoms are represented by grey, green, and purple spheres, respectively.
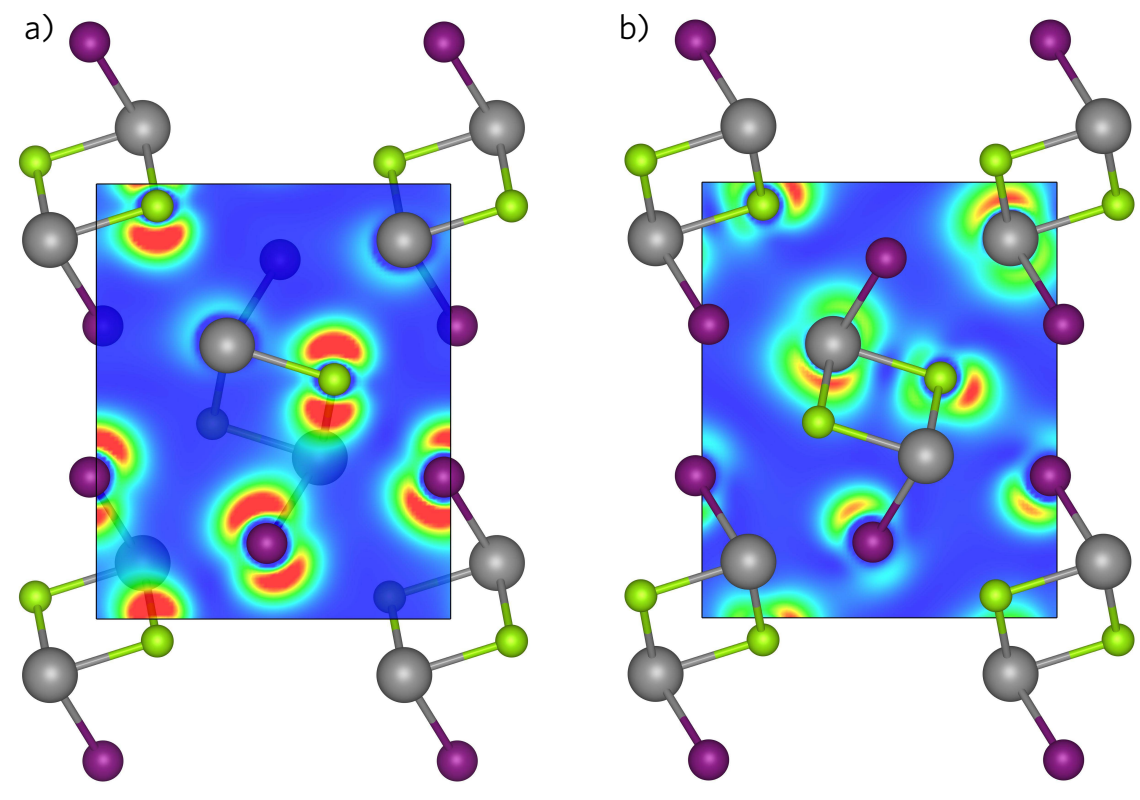

\section{Effective masses and dielectric constants}

The effective masses at the band edges of the VBM $\left(m_{\mathrm{h}}^{*}\right)$ and CBM $\left(m_{\mathrm{e}}^{*}\right)$ are provided in Table 8.2. The electron effective masses are significantly larger for the directions between the $\left[(\mathrm{BiChI})_{\infty}\right]_{2}$ ribbons (between $\Gamma$ and $Z$, along [001]), highlighting the weak nature of the dispersive interactions holding the ribbons together. For directions parallel to the ribbons ( $\Gamma \rightarrow$ Y, along [100]), the effective masses are much smaller, suggesting that conductivity will be strongly anisotropic and highlighting the potential for significantly increased carrier mobilities in devices containing vertically oriented crystals. BiSeI possesses the smallest electron effective masses, with these occurring in the direction from $\Gamma$ to $\mathrm{Y}\left(0.51 \mathrm{~m}_{0}\right)$, whereas the smallest electron effective masses in BiSI appear in the direction from the CBM toward $\mathrm{Z}\left(0.69 \mathrm{~m}_{0}\right)$. In both compounds the hole effective masses are small, suggesting that the short hole diffusion lengths seen experimentally in BiSI may be the result of scattering and impurity effects. ${ }^{319}$ Interestingly, in both cases the hole effective masses are lower than the electron effective masses. This is similar to in MAPI ${ }^{22}$ and likely results from the presence of anti-bonding Bi $s$ states at the VBM. The values obtained for the effective masses are slightly larger than in 
MAPI $\left(m_{\mathrm{e}}^{*}=0.15 \mathrm{~m}_{0} ; m_{\mathrm{h}}^{*}=0.12 \mathrm{~m}_{0}\right)^{22}$ but comparable to other bismuth containing solar absorbers such as $\mathrm{Bi}_{2} \mathrm{~S}_{3}\left(m_{\mathrm{e}}^{*}=0.25 \mathrm{~m}_{0} ; m_{\mathrm{h}}^{*}=\right.$ $\left.0.44 \mathrm{~m}_{0}\right) .{ }^{336}$

As previously mentioned, large dielectric constants $\left(\varepsilon_{\mathrm{r}}\right)$ are considered a primary factor in the excellent performance seen in the hybrid perovskites. ${ }^{9,22,40}$ The dielectric response of a material is crucial in determining the charge capture cross-section of defects, with more effective charge screening resulting in smaller cross sections and lower recombination and scattering rates. ${ }^{19}$ Additionally, larger dielectric constants should promote smaller defect binding energies (in effective mass theory $E_{\mathrm{b}}=m_{\mathrm{e}}^{*} / 2 \varepsilon_{\mathrm{r}}^{2}$ ), ${ }^{22,337}$ preventing defect states from appearing deep within the gap. As such, we have calculated the dielectric constants of both materials, finding them to be 36.8 and 35.8 for BiSI and BiSeI, respectively (Table 8.2). While smaller than those seen in the lead hybrid halide perovskites - where dielectric constants can reach $60-70$ or higher, thanks to screening provided by the organic cation 221,236 - they are noticeably larger than in other established absorbers such as CZTS ( 9). ${ }^{222}$ We therefore expect this combination of small effective masses and large dielectric constants to promote shallower defect states and reduced levels of charge-carrier recombination. ${ }^{338}$

\section{Alignments with electron and hole contact layers}

Band alignments calculated using HSE06+SOC are provided in Figure 8.6. The ionisation potentials (IPs) of BiSI and BiSeI are significantly larger than many established photovoltaic absorbers $(6.4 \mathrm{eV}$ and $6.2 \mathrm{eV}$ below the vacuum level, respectively). ${ }^{22,90,339}$ It is clear that the band gap reduction when moving from BiSI to BiSeI results from the smaller ionisation potential of BiSeI, combined with concomitant stabilisation of the conduction band minimum (an increase in the electron affinity). Figure 8.6 also details the band alignments obtained experimentally by Hahn et al., which show an increase in the ionisation potential when $\mathrm{S}$ is replaced with $\mathrm{Se}$ (from $\sim 6.1 \mathrm{eV}$ in BiSI to $\sim 6.3$ in BiSeI). ${ }^{318}$ This trend is unexpected as the increase in energy of the Ch $p$ orbital down the chalcogen group typically results
TABLE 8.2: Band gaps, effective masses and low-frequency dielectric constants for BiSI and BiSel, calculated using HSEO6+SOC. Effective masses given in units of $m_{0}$

\begin{tabular}{lcc}
\hline & BiSI & BiSeI \\
\hline$E_{\mathrm{g}}^{\text {ind }}(\mathrm{eV})$ & 1.78 & 1.50 \\
$E_{\mathrm{g}}^{\text {dir }}(\mathrm{eV})$ & 1.82 & 1.60 \\
$m_{\mathrm{e}}^{*}(\mathrm{CBM} \rightarrow \mathrm{Z})$ & 0.69 & 4.52 \\
$m_{\mathrm{e}}^{*}(\mathrm{CBM} \rightarrow \Gamma)$ & 1.78 & - \\
$m_{\mathrm{e}}^{*}(\mathrm{CBM} \rightarrow \mathrm{Y})$ & - & 0.51 \\
$m_{\mathrm{h}}^{*}(\mathrm{VBM} \rightarrow \Gamma)$ & 0.51 & - \\
$m_{\mathrm{h}}^{*}(\mathrm{VBM} \rightarrow \mathrm{Y})$ & 0.36 & - \\
$m_{\mathrm{h}}^{*}(\mathrm{VBM} \rightarrow \mathrm{Z})$ & - & 0.40 \\
$m_{\mathrm{h}}^{*}(\mathrm{VBM} \rightarrow \Gamma)$ & - & 0.28 \\
$\varepsilon_{\mathrm{r}}$ & 36.8 & 35.8 \\
\hline
\end{tabular}


FIGURE 8.6: HSE06+SOC calculated band alignment of BiSI and BiSel with a series of transparent conducting oxides and hole transporting materials, taken from experiment. $232,343-347$ Electron affinities calculated using the experimental band gap and HSEO6+SOC ionisation potentials are show in dashed red lines. Experimental band alignments performed by Hahn et al. are also plotted for comparison. ${ }^{318}$ Experimental uncertainty is indicated by error bars.

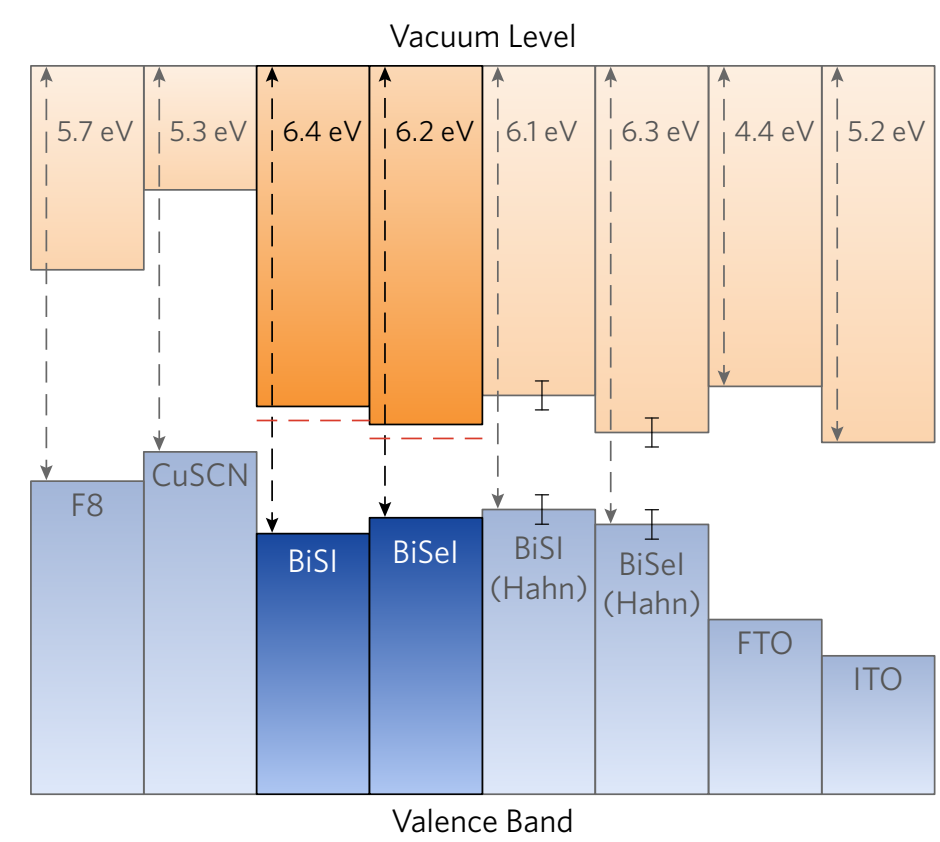

in a raising of the valence band maximum and decrease in the ionisation potential, as observed in many other mixed chalcogenide systems including $\mathrm{CdS}_{1-x} \mathrm{Se}_{x},{ }^{340} \mathrm{CdSe}_{x} \mathrm{Te}_{1-x},{ }^{341}$ and $\mathrm{Cu}_{2} \mathrm{ZnSn}(\mathrm{S}, \mathrm{Se})_{4} \cdot{ }^{342}$ This discrepancy may be rationalised through the authors' observation of BiOI contamination on the surface of their samples. ${ }^{318}$

Band alignments for a range of commonly used transparent conducting oxides (TCOs) and hole transporting materials (HTMs) are also included in Figure 8.6. It is clear that the materials used in the photovoltaic devices constructed by Hahn et al. (namely FTO and $p$-CuSCN) result in band misalignments against both the TCO and HTM layers. ${ }^{318}$ For example, when the electron affinity of BiSI is calculated based on the HSE06+SOC ionisation potential and experimentally measured band gap, the maximum obtainable open-circuit voltage from Hahn et al.'s device is limited to just $\sim 0.4 \mathrm{eV} .{ }^{343,344} \mathrm{An}$ alternative HTM with a lower ionisation potential, such as poly(fluorene2,7-diyl) (F8, IP = 5.7 eV), ${ }^{345}$ will therefore allow for significantly larger open-circuit voltages and increased power conversion efficiencies. When considering the TCO layer, the electron affinities of BiSI and $\mathrm{BiSeI}(\mathrm{EA}=4.9 \mathrm{eV}$ and $5.0 \mathrm{eV}$, respectively) are larger than that of FTO $(\mathrm{EA} \simeq 4.4 \mathrm{eV}),{ }^{232}$ indicating that electron transfer from the absorber to the $n$-type layer will be poor. TCOs with larger electron 
affinities - for example indium tin oxide $(\text { ITO, } \mathrm{EA} \simeq 5.2 \mathrm{eV})^{346,347}$ - should therefore be employed to enable more efficient transport of photo-generated carriers. We note that the above analysis relies solely on the fundamental band alignments of the bulk crystal and does not take into account effects originating from interfacial strain and other chemical interactions. Regardless, it is clear that the use of revised contact materials will be essential if device efficiencies are to improve.

\section{Defect chemistry}

As BiSI and BiSeI show promising electronic properties for photovoltaic applications, we have performed calculations to investigate how their defect behaviour will influence device performance. The chemical potential phase diagram of both compounds is provided in Figure 8.7. The thermodynamically accessible range of chemical potentials are bounded by the formation of the binary bismuth chalcogenides $\left(\mathrm{Bi}_{2} \mathrm{~S}_{3}\right.$ and $\left.\mathrm{Bi}_{2} \mathrm{Se}_{3}\right)$ and $\mathrm{BiI}_{3}$, resulting in a narrow but broad region spanning a significant portion of chemical potential space. In each case, two synthetic environments were identified: the most p-type environment, A, shows bismuth-poor and anion-rich (sulfur and iodine-rich) conditions; whereas the most $n$-type, $\mathrm{B}$, is anion-
FIGURE 8.7: Range of accessible chemical potentials for a) BiSI and b) BiSel.

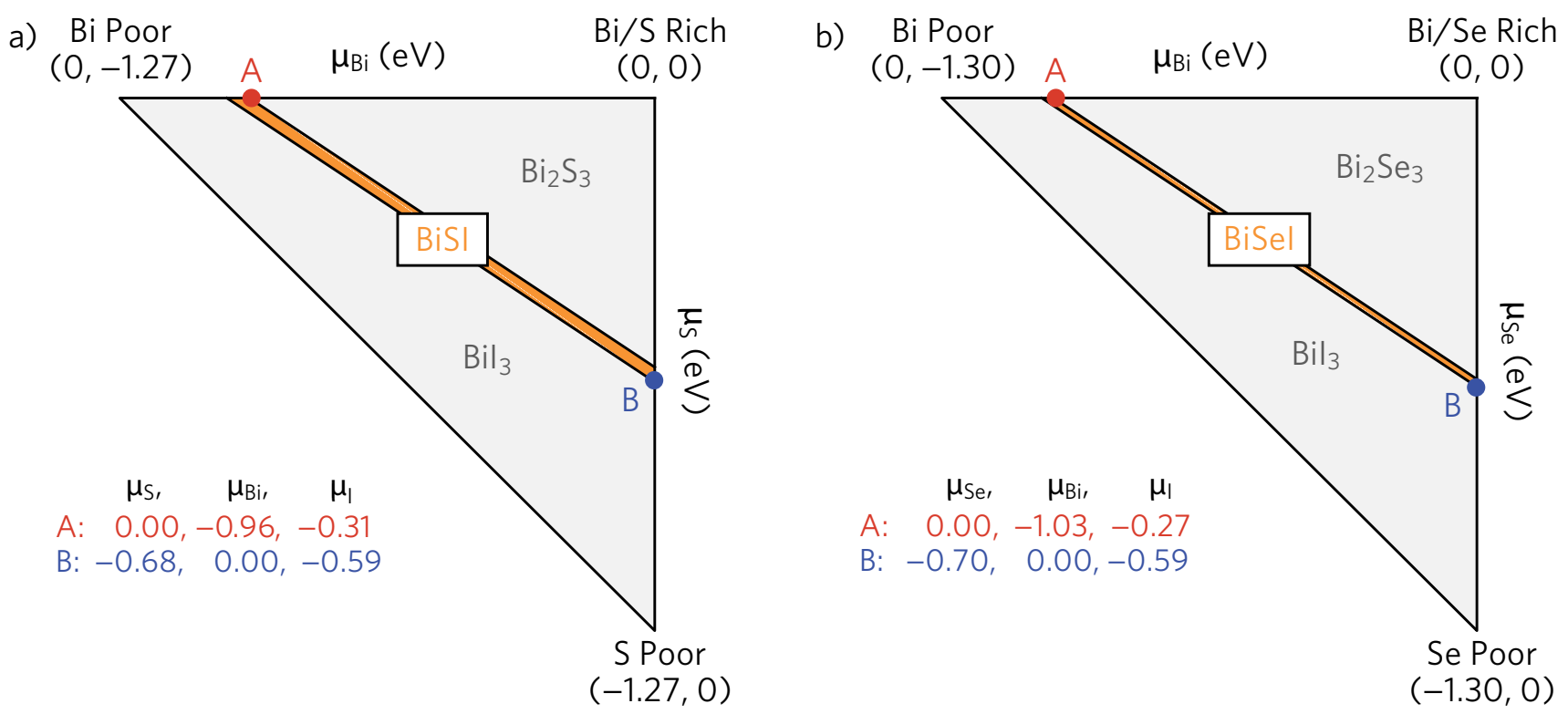



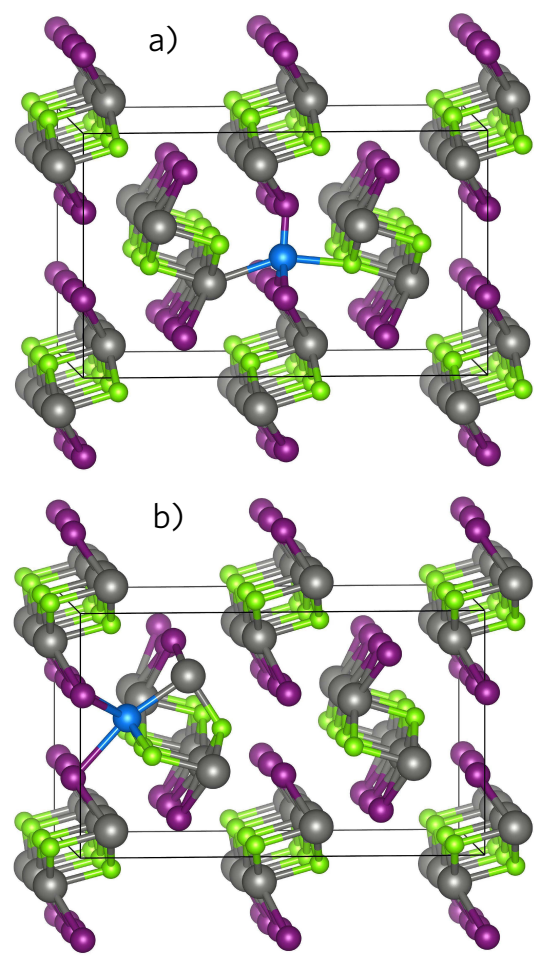

FIGURE 8.8: Crystal structure of the $2 \times 3 \times 1$ supercell of BiSI used in defect calculations, indicating the (a) penta-coordinated and (b) octa-coordinated interstitial sites. Bi, S, and I atoms are denoted by grey, green, and purple spheres, respectively. Interstitial atom denoted by a blue sphere.

FIGURE 8.9: Defect formation energies as a function of Fermi level for BiSI under a $p$-type (Bipoor, $\mathrm{S}$ and I-rich) chemical potential environment. The slope of the lines denotes the charge state, with a steeper line indicating a higher charge state. The solid dots represent the transition levels, $\varepsilon\left(q / q^{\prime}\right)$. poor and bismuth-rich.

In this study, the $n$-type defects investigated were anion vacancies $\left(V_{\mathrm{I}}\right.$ and $\left.V_{\mathrm{Ch}}\right)$, iodine on chalcogen antisite $\left(\mathrm{I}_{\mathrm{Ch}}\right)$, anion on bismuth antisites $\left(\mathrm{Ch}_{\mathrm{Bi}}\right.$, and $\left.\mathrm{I}_{\mathrm{Bi}}\right)$, and bismuth interstitials $\left(\mathrm{Bi}_{i}\right)$. For $p$-type defects, we have considered a bismuth vacancy $\left(V_{\mathrm{Bi}}\right)$, chalcogen on iodine antisites $\left(\mathrm{Ch}_{\mathrm{I}}\right)$, bismuth on anion antisites $\left(\mathrm{Bi}_{\mathrm{Ch}}\right.$ and $\left.\mathrm{Bi}_{\mathrm{I}}\right)$, and anion interstitials $\left(\mathrm{Ch}_{i}\right.$ and $\left.\mathrm{I}_{i}\right)$. Two interstitial defect sites were located in the voids between the one-dimensional ribbons, comprising one penta-coordinated site and one octa-coordinated site (Fig. 8.8).

\section{BiSI defects}

The defect formation energies as a function of Fermi level, for the intrinsic defects of BiSI, under the most $p$-type conditions (environment A), are provided in Figure 8.9. Due to the large number of defect states, the interstitial defects are plotted separately from the vacancies and antisites. The lowest energy acceptor, $S_{I}$, possesses a relatively shallow $0 /-1$ transition level $0.14 \mathrm{eV}$ above the valence band maximum. This is compensated by the lowest energy donor defects, $\mathrm{I}_{S}$ and $V_{\mathrm{I}}$, which will act to trap the Fermi level in the middle of the band gap. This is confirmed by calculation of the self-consistent

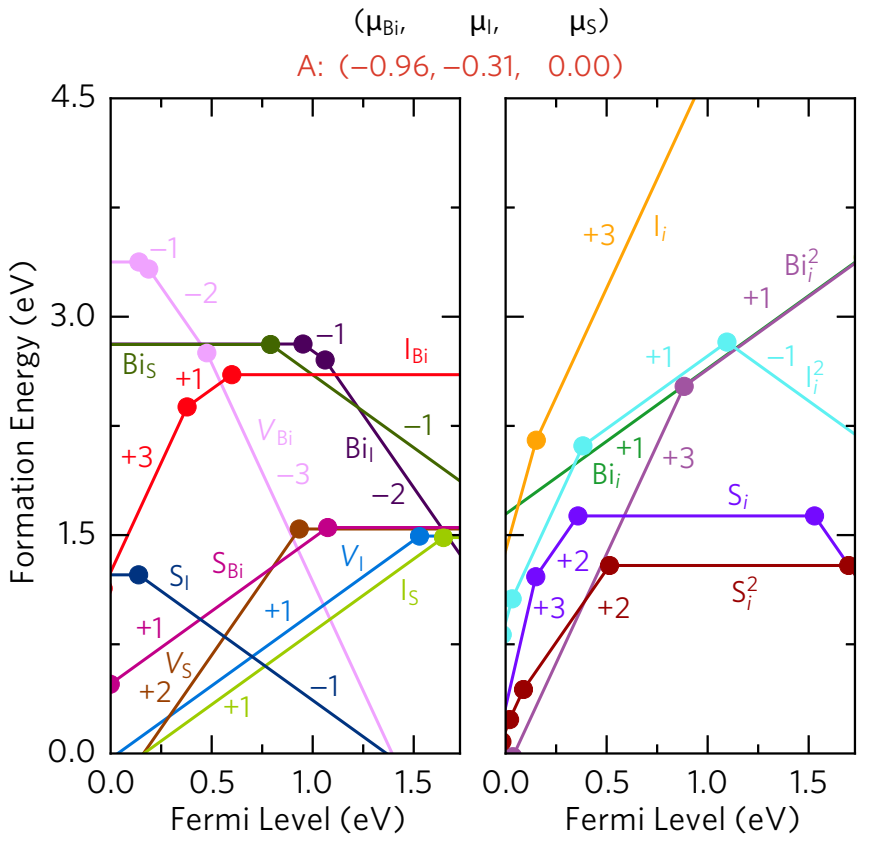


Fermi level (at a temperature of $21^{\circ} \mathrm{C}$ ), which indicates the Fermi level will be pinned $0.77 \mathrm{eV}$ above the valence band maximum, resulting in a hole concentration of $1.21 \times 10^{8} \mathrm{~cm}^{-3}$.

The remainder of the acceptor antisite defects possess large formation energies (around $3 \mathrm{eV}$ for the neutral charge states), which will preclude their formation at the low processing temperatures required to synthesise BiSI thin films. ${ }^{348}$ Considering the interstitial defects: $\mathrm{I}_{i}^{2}, \mathrm{~S}_{i}$, and $\mathrm{S}_{i}^{2}$ may act as acceptors provided the Fermi level sits close to the conduction band minimum. As the Fermi level under environment A will be trapped in the middle of the band gap, these defects will therefore not contribute to $p$-type conductivity.

The $\mathrm{S}_{\mathrm{Bi}}$ and $\mathrm{S}_{i}^{2}$ donor defects show ultra-deep states in the middle of the gap, which could contribute to trap-assisted charge-carrier recombination. Analysis of the self-consistent Fermi level, however, reveals that these defects will be present at remarkably low concentrations $\left(5.8 \times 10^{1} \mathrm{~cm}^{-3}\right.$ and $0.8 \times 10^{1} \mathrm{~cm}^{-3}$, respectively, calculated at a representative device operating temperature of $21^{\circ} \mathrm{C}$ ) and will therefore have a limited effect on $p$-type conductivity.

The transition level diagram for the intrinsic defects of BiSI under the most $n$-type conditions (environment $\mathrm{B}$ ), is provided in Figure 8.10. $\mathrm{I}_{\mathrm{S}}$ is the lowest energy donor defect, with a shallow $\%+1$ tran-

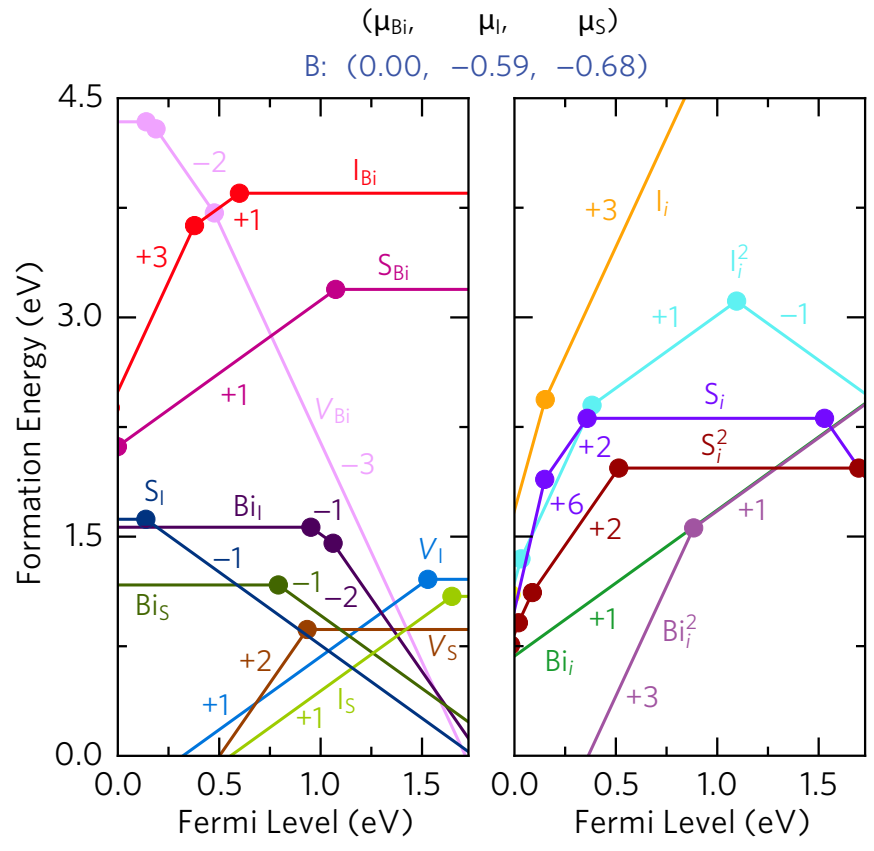

FIGURE 8.10: Defect formation energies as a function of Fermi level for BiSI under an $n$-type (Birich, $\mathrm{S}$ and I-poor) chemical potential environment. The slope of the lines denotes the charge state, with a steeper line indicating a higher charge state. The solid dots represent the transition levels, $\varepsilon\left(q / q^{\prime}\right)$. 
a)
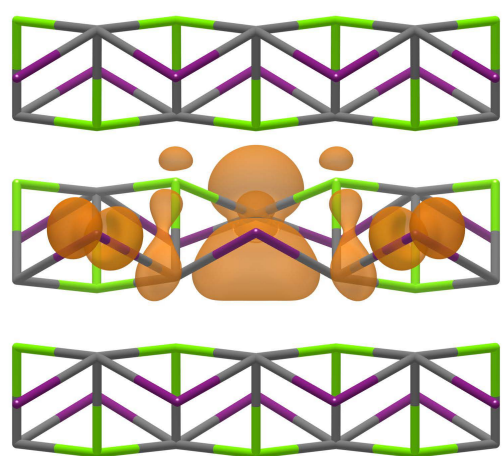

b)
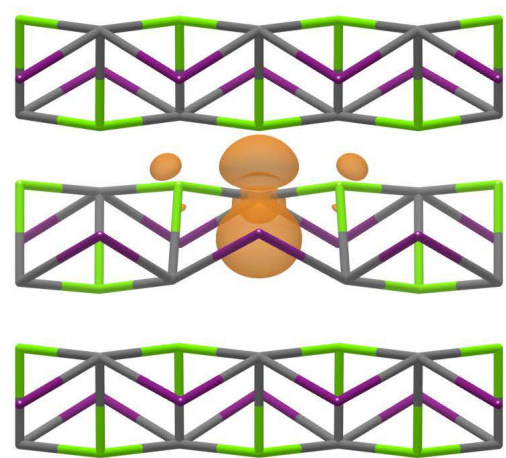

c)
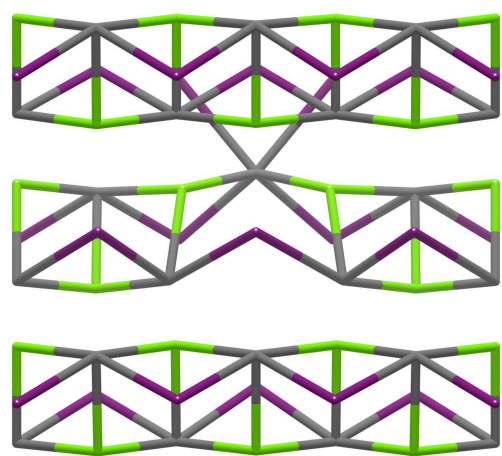

FIGURE 8.11: Crystal structure and charge density isosurfaces of the $V_{S}$ defect in the a) neutral, b) +1 , and c) +2 charge states, viewed along the [001] direction. Green, grey and purple cylinders indicate bonding to sulfur, bismuth and iodine atoms, respectively. Electron density is represented in orange. The isosurface level was set to $0.03 \mathrm{eV} \AA^{-3}$. sition level $0.08 \mathrm{eV}$ beneath the conduction band minimum. The lowest energy acceptor defect, $\mathrm{S}_{\mathrm{I}}^{-1}$, again causes the Fermi level to be trapped mid-gap ( $0.57 \mathrm{eV}$ below the conduction band edge). $V_{\mathrm{I}}$ is also a low energy donor but possesses a deeper $0 /+1$ transition state $0.20 \mathrm{eV}$ from the conduction band minimum. The neutral $V_{\mathrm{I}}$ is higher in energy than the neutral $V_{\mathrm{S}}$, however, the sulfur vacancy is a negative$\mathrm{U}$ defect, possessing an ultra-deep $0 /+2$ transition state in the middle of the band gap. Examination of the charge-density isosurfaces for each charge state reveals the origin of this behaviour: in the neutral state, the two excess electrons are delocalised over the three bismuth atoms closest to the defect site, with the bulk of the electron density occupying the void in the structure (Fig. 8.11a). This arrangement is stabilised by a slight displacement of the bismuth atom opposite the defect site, which is pulled forward into the vacancy, allowing the rest of the one-dimensional ribbon to remain largely unperturbed. Moving to the +1 charge state, the remaining electron is localised solely on the bismuth atom opposite the defect site, with significantly less electron density occupying the void (Fig. 8.11b). As the two bismuth atoms either side of the vacancy are no longer hybridised, they are pulled outward, causing a high-energy structural distortion that travels the length of the one-dimensional chain, thereby increasing the strain in the lattice. In the +2 charge state, the bismuth opposite the vacancy site is displaced backwards as it bonds with two iodine atoms in an nearby ribbon (Fig. 8.11c). Overall, the distortion of this geometry is comparable to the +1 charge state, however, the additional bonding provides greater structural stability. 
$\mathrm{Bi}_{i}^{2}$ is the lowest energy donor interstitial, possessing an ultradeep $+1 /+3$ transition state, however, its high formation energy at the self-consistent Fermi level indicates it will only be present in very low concentrations. It should be noted that both $V_{\mathrm{S}}$ and $\mathrm{Bi}_{\mathrm{S}}$ are low energy, ultra-deep defects that may contribute to charge-carrier recombination. As such, post-annealing in a sulfur atmosphere should be considered to reduce the effect on $n$-type conductivity.

\section{BiSeI defects}

The defect chemistry of BiSeI is similar to that of its sulfur counterpart. The defect formation energies as a function of Fermi level, for the intrinsic defects of BiSeI, under the most $p$-type conditions (environment $\mathrm{A}$ ), is provided in Figure 8.12. $\mathrm{Se}_{\mathrm{I}}$ is the lowest energy acceptor defect, possessing a transition level $0.13 \mathrm{eV}$ above the valence band maximum. In comparison to the equivalent defect in BiSI $\left(\mathrm{S}_{\mathrm{I}}\right)$, $\mathrm{Se}_{\mathrm{I}}$ is both lower in energy and more shallow. The lowest energy donor defect is $\mathrm{Se}_{\mathrm{I}}$, which will trap the Fermi level $0.68 \mathrm{eV}$ above the valence band, resulting in a hole concentration of $8.52 \times 10^{7} \mathrm{~cm}^{-3}$ at $21^{\circ} \mathrm{C}$.

The main variation between the BiSeI and BiSI $p$-type defects is

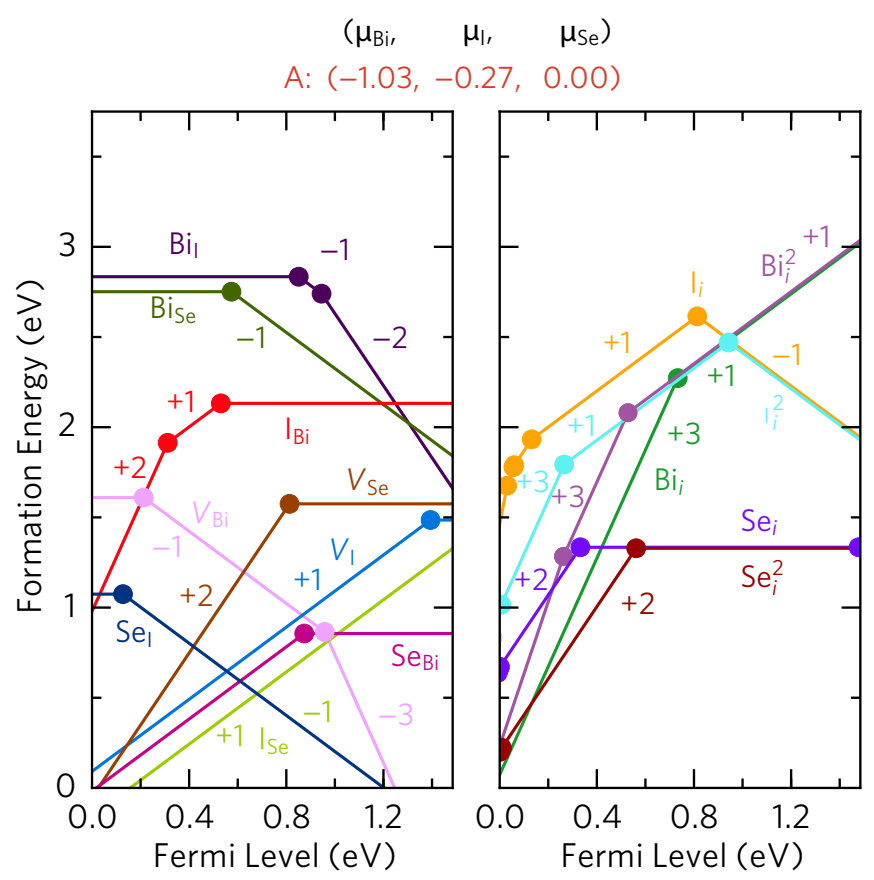

FIGURE 8.12: Defect formation energies as a function of Fermi level for BiSel under a $p$-type (Bipoor, Se and I-rich) chemical potential environment. The slope of the lines denotes the charge state, with a steeper line indicating a higher charge state. The solid dots represent the transition levels, $\varepsilon\left(q / q^{\prime}\right)$. 
FIGURE 8.13: Defect formation energies as a function of Fermi level for BiSel under an $n$-type (Birich, Se and I-poor) chemical potential environment. The slope of the lines denotes the charge state, with a steeper line indicating a higher charge state. The solid dots represent the transition levels, $\varepsilon\left(q / q^{\prime}\right)$. found for $\mathrm{Se}_{\mathrm{Bi}}$, which possesses a significantly reduced formation energy (enabling high defect concentrations of $4.77 \times 10^{10} \mathrm{~cm}^{-3}$ ) and an ultra-deep $\%+1$ transition level. As such, this defect may play a role in trap-assisted recombination. Similar to BiSI, the remaining interstitial and antisite defects show significantly higher formation energies, which preclude their formation in high concentrations.

The transition level diagram for the intrinsic defects of BiSeI under the most $n$-type conditions (environment $\mathrm{B}$ ) is provided in Figure 8.13. In general, the larger electron affinity of BiSeI results in donor defects that are more shallow than in the sulfur analogue. ISe is the lowest energy donor defect, possessing a transition level resonant in the conduction band $(0.17 \mathrm{eV}$ above the conduction band edge). This is compensated by both $\mathrm{Bi}_{\mathrm{Se}}$ and $\mathrm{Se}_{\mathrm{I}}$, causing the Fermi level to be pinned $0.43 \mathrm{eV}$ beneath the conduction band minimum, producing an electron concentration of $7.64 \times 10^{11} \mathrm{~cm}^{-3}$ at $21^{\circ} \mathrm{C}$.

Similar to BiSI, both $\mathrm{Bi}_{\mathrm{Se}}$ and $V_{\mathrm{Se}}$ possess ultra-deep transition levels, with relatively low formation energies and self-consistent concentrations of $4.84 \times 10^{12} \mathrm{~cm}^{-3}$ and $1.17 \times 10^{7} \mathrm{~cm}^{-3}$, respectively. As such, annealing in a selenium atmosphere may be required to alleviate any negative effects on charge-carrier recombination. In all cases, the interstitial defects possess large formation energies, and will not

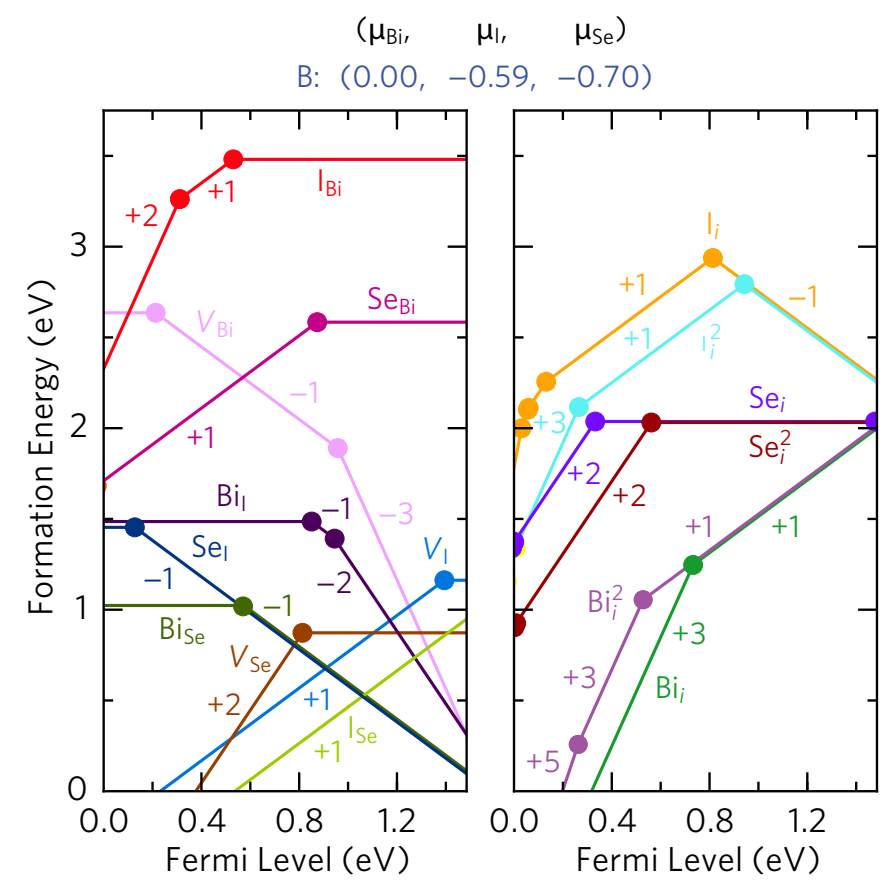


be present in high concentrations at standard device operating conditions.

\section{Defect engineering}

Under both $n$ - and $p$-type conditions, the Fermi level of BiSI and BiSeI will be trapped mid gap. As such, both compounds will behave as intrinsic semiconductors and are most well suited to use in a $p-i-n$ junction solar cell architecture. The transition level diagrams reveal the existence of low energy deep defects (for example $\mathrm{Ch}_{\mathrm{Bi}}$ and $\left.V_{\mathrm{Ch}}\right)$, present in reasonably high concentrations, at the limits of both $n$ - and $p$-type synthesis conditions. As previously discussed, these defect sites are likely to contribute to trap-assisted recombination, and may have a detrimental effect on the open circuit voltage (see Section 1.2.2). In order to minimise the effect of these defects on recombination, we have explored the defect behaviour across the full range of achievable chemical potentials. Using the self-consistent Fermi level method, we have calculated the charge-carrier and defect concentrations for each point within the region of stability, identified in the chemical potential phase diagrams shown in Figure 8.7. Due to the low carrier concentrations present, Shockley-Read-Hall recombination will be the dominant recombination mechanism in BiSI and BiSeI devices. ${ }^{116}$ Accordingly, we have calculated an estimate of the recombination rate, at each set of chemical potentials, using the Shockley-Read-Hall recombination model discussed in Section 3.4.6. Here, the recombination rate, $R^{\mathrm{SRH}}$, is dependent on the concentration of holes and electrons, in addition to the number of defects and the positions of the charge transition levels.

In this model, defect states closer to the centre of the band gap show exponentially greater activity as recombination centres than states located close to the valence band maximum or conduction band minimum. In this way, as the number of shallow versus deep defects changes across chemical potential space, the overall rate of recombination will also vary. As calculating defect charge-capture cross sections from first-principles is extremely computationally demanding, ${ }^{349}$ we have instead assumed a constant cross-section for all de- 


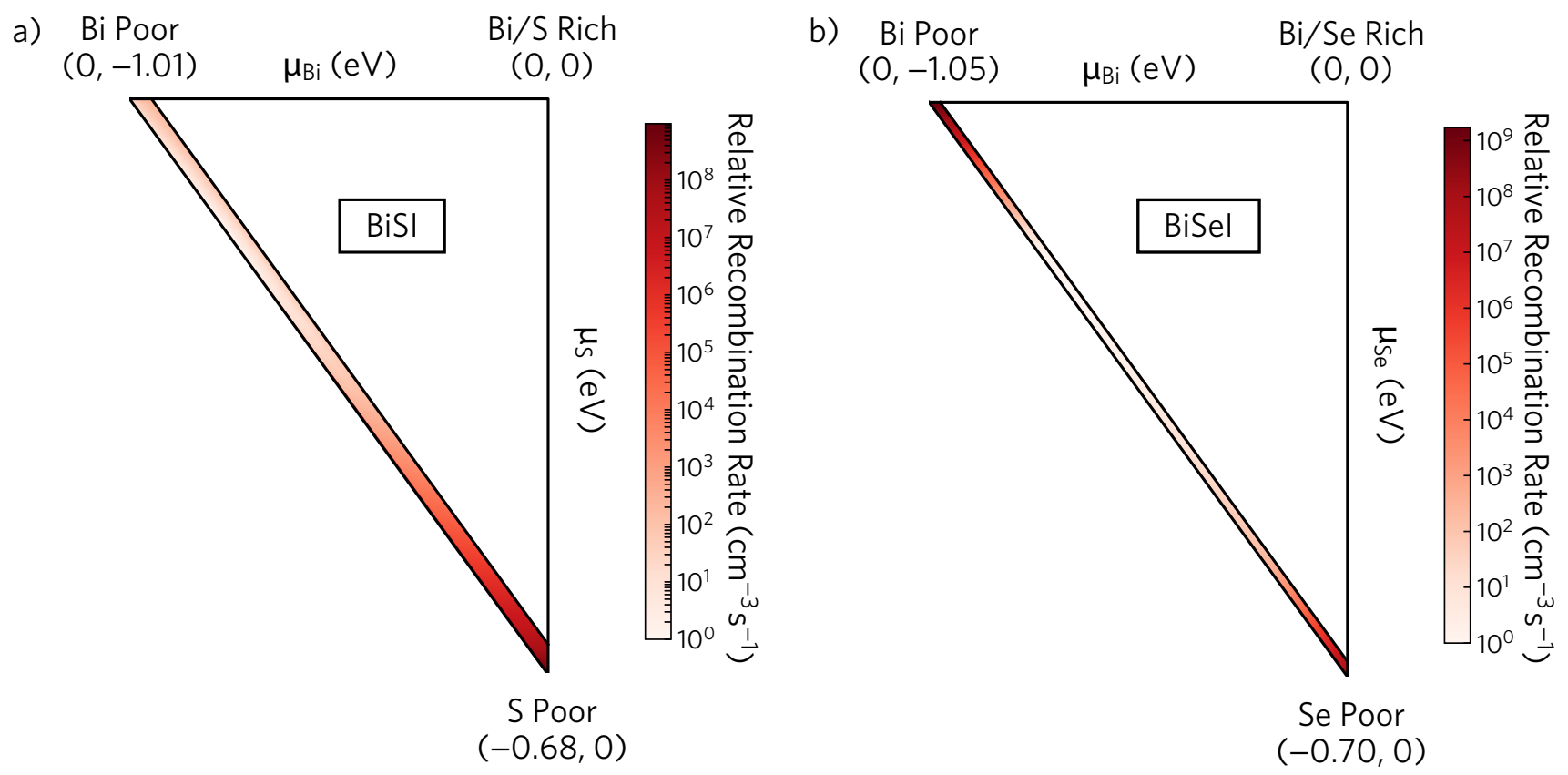

FIGURE 8.14: Shockley-ReadHall recombination rate $\left(R_{\mathrm{SRH}}\right)$ across stability region in chemical potential space for a) BiSI and b) BiSel. Larger recombination rates are indicated by darker red regions. fects $\left(\sigma=1 \times 10^{-15} \mathrm{~cm}^{2}\right)$. Accordingly, our results will form an qualitative estimate of the true recombination rate.

The Shockley-Read-Hall recombination rate, as a function of chemical potential, is provided in Figure 8.14. We have normalised the data to the region of lowest recombination. Considering BiSI: the $p$-type conditions possess the lowest levels of recombination, resulting from the high defect formation energy of the most prevalent deep defects $\left(V_{\mathrm{S}}\right.$ and $\left.\mathrm{S}_{\mathrm{Bi}}\right)$. The recombination rate increases gradually as the chemical potentials shift to favouring the formation of $n$-type defects, due to the reduction in formation energy of the ultra-deep $\mathrm{Bi}_{\mathrm{S}}$ and $V_{\mathrm{S}}$ defects. Under the limits of $n$-type conductivity, the recombination rate is over $10^{7} \mathrm{~s}^{-1}$ more than under $p$-type conditions. As such, bismuth poor synthesis conditions should be considered to minimise the levels of recombination, and maintain an optimal open-circuit voltage.

In contrast, BiSeI shows high levels of recombination under the limits of both $n$ - and $p$-type synthesis conditions. This can be attributed to the low formation energy of the ultra-deep $V_{\mathrm{Se}}$ and $\mathrm{Bi}_{\mathrm{Se}}$ defects - which dominate under the most $n$-type environment and the $\mathrm{Se}_{\mathrm{Bi}}$ defect - which is present under the most $p$-type envi- 
ronment. In the middle of the chemical potential space, however, the recombination rate is reduced considerably, due to the absence of any low-energy deep defect states. Accordingly, use of a more stoichiometric ratio of starting materials will likely limit the formation of "killer-defects" in high concentrations.

\section{Conclusions}

In this chapter, we have investigated BiSI and BiSeI as potential solar absorber materials. Using first-principles relativistic electronic structure theory, we show that BiSI and BiSeI possess ideal electronic structures for photovoltaic devices, with band gaps in the optimal range specified by the Shockley-Queisser limit. Small effective masses in the directions parallel to the one-dimension ribbons, coupled with large dielectric constants, are expected to promote charge transport and reduce the effects of charged-defect scattering. Based on analysis of the electronic band alignments, we have identified band misalignments as a potential cause of the poor efficiencies of BiSI and BiSeI devices previously reported in the literature. Accordingly, we have suggested alternative electron and hole contact materials that we expect will result in improved open-circuit voltages, charge-carrier extraction, and device efficiencies. By calculating the defect properties of both compounds, we identify these materials as intrinsic semiconductors best suited for use in a $p-i-n$ junction solar cell architecture. Through mapping the rate of Shockley-Read-Hall recombination across chemical potential space, we further reveal regions showing reduced recombination rates. As such, judicious choice of synthesis conditions will be essential in producing devices with optimal trap-assisted recombination properties. These results will be beneficial for experimental groups aiming to produce efficient bismuth chalcohalide photovoltaic devices in the future.

\section{Notes}

The work discussed in this chapter formed the basis for two publications: 
1. A. M. Ganose, K. T. Butler, A. Walsh and D. O. Scanlon, Relativistic Electronic Structure and Band Alignment of BiSI and BiSeI: Candidate Photovoltaic Materials, Journal of Materials Chemistry A, 4, 2060 (2016)

2. A. M. Ganose, S. Matsumoto, J. Buckeridge, and D. O. Scanlon, Defect Engineering of Earth-Abundant Solar Absorbers BiSI and BiSeI, Chemistry of Materials, 30, 3827-3835 (2018)

The optimised crystal structures, for all compounds discussed in this chapter, are provided in an online repository. ${ }^{321}$ 


\section{SUMmARY}

The aim of this thesis was to critically assess the fundamental properties of emerging photovoltaic absorbers through use of first-principles electronic structure calculations. It is currently impossible to work in the field of photovoltaics without acknowledging the success of the hybrid perovskites, which have seen truly impressive efficiency gains in the last nine years. Despite considerable research attention, however, the hybrid perovskites present several challenges which remain to be overcome. The materials examined in this thesis have all emerged in response to these challenges. In order to evaluate the potential of these materials as photovoltaic absorbers, an in-depth understanding of their atomic-scale properties is essential. In practice, this means not only understanding bulk optoelectronic properties but also the role of defects, which will play a major role on carrier transport and recombination. In this chapter, we summarise the major findings from our work and highlight future research directions.

In Part 2 of this thesis we looked at absorbers derived from modifications to the perovskite structure. In recent months, the layered compound, $\left(\mathrm{CH}_{3} \mathrm{NH}_{3}\right)_{2} \mathrm{~Pb}(\mathrm{SCN})_{2} \mathrm{I}_{2}$ (MAPSI), has been proposed as a more stable alternative to the cubic perovskite $\mathrm{CH}_{3} \mathrm{NH}_{3} \mathrm{PbI}_{3}$, however the origin of this stability was unknown. By examining the energetics of decomposition for both compounds, we demonstrated that MAPSI is intrinsically more stable against decomposition via several routes. To investigate MAPSI's photovoltaic performance, we performed relativistic density functional theory calculations. These revealed MAPSI possesses a band gap suitable for a top cell in a photovoltaic tandem device, along with small hole and electron effective masses that should enable high carrier mobilities. Furthermore, the 
majority of intrinsic vacancy defects are shallow and therefore should not adversely affect carrier recombination rates.

We demonstrated that, similar to the $\mathrm{ABX}_{3}$ perovskites which can be tuned on the A, B and X sites, MAPSI can also act as a parent compound to a range of MAPSI-structured analogues. Replacing SCN with OCN and SeCN, I with $\mathrm{Cl}$ and $\mathrm{Br}$, and $\mathrm{Pb}$ with $\mathrm{Sn}$, enables a series of compounds with a diverse range of electronic properties. The band gap, valence bandwidth, and charge-carrier effective masses of these analogues are determined by the relative energies of the halide and pseudohalide $p$ states. Furthermore, all analogues are stable with respect to decomposition. The iodide analogues all possess band gaps suitable for photovoltaic top cells and are therefore of interest to the photovoltaic community. In particular, the tin iodides present an exciting possibility for the future of stable lead-free perovskites. We therefore welcome the experimental realisation of these promising materials. Future work may focus on the possibility of quasi-2D MAPSI structured perovskites. Indeed, as layered perovskites show dramatic efficiency and stability improvements when the layer thickness is greater than 1 perovskite sheet, so too may the MAPSI analogues. This may provide a mechanism to further lower the band gap and bring it into the range suitable for single-junction photovoltaics.

The toxicity of lead remains the second major concern surrounding the hybrid perovskites. In Chapter 6 we investigated the leadfree vacancy-ordered defect perovskites $\mathrm{Cs}_{2} \mathrm{SnI}_{6}$ and $\mathrm{Cs}_{2} \mathrm{TeI}_{6}$. We demonstrated that while $\mathrm{Cs}_{2} \mathrm{SnI}_{6}$ possesses a direct band gap, it is dipole disallowed and therefore results in weak optical absorption. Accordingly, we provided an explanation for the apparent mismatch between theory and experiment previously discussed in the literature. Furthermore, the fundamental band gap of $\mathrm{Cs}_{2} \mathrm{TeI}_{6}$ is indirect and so neither compounds are expected to perform well as photovoltaic absorbers. Despite this, we showed that both contain intriguing electronic structures deserving of further study. In particular, the presence of dispersive conduction band states containing significant Sn contributions, despite the presence of regular vacancies, is due to 
inter-octahedral I-I interactions that span the voids in the lattice.

We showed that the dramatic reduction in conductivity seen by our experimental collaborators upon tellurium substitution in the series $\mathrm{Cs}_{2} \mathrm{Sn}_{1-x} \mathrm{Te}_{x} \mathrm{I}_{6}$, cannot be explained by differences in carrier effective masses. Instead, this behaviour is due to the depth of iodine vacancy defects, which are shallowest for $\mathrm{Cs}_{2} \mathrm{SnI}_{6}$ and become deeper with increasing tellurium incorporation. To overcome the primary limitation of these materials in the context of photovoltaics - i.e. the dipole disallowed fundamental band gap - it may be fruitful to investigate $\mathrm{Cs}_{2} \mathrm{SnI}_{x} \mathrm{Br}_{6-x}$ solid-solutions, with the aim of breaking the symmetry of the frontier orbitals and restoring strong optical absorption.

In Part 3 we looked for photovoltaic absorbers beyond the hybrid perovskite family. Bismuth-based materials were identified as attractive candidate absorbers due to their earth-abundant and non-toxic nature. We demonstrated that BiSI and BiSeI possess ideal electronic structures for photovoltaic devices, with band gaps in the optimal range specified by the Shockley-Queisser limit. Their small effective masses and large dielectric constants should further promote efficient charge transport and reduce the effects of charged-defect scattering. We demonstrated that band misalignments are most likely the cause of the poor efficiencies of BiSI and BiSeI devices previously reported in the literature. Furthermore, we calculated their defect properties and showed both compounds are best suited for use in $p-$ $i-n$ junction solar cell architectures. Lastly, we demonstrated that judicious choice of synthesis conditions should enable dramatic reductions in the rate of Shockley-Read-Hall recombination. We believe these results will aid the fabrication of more efficient bismuth chalcohalide photovoltaic devices. Future theoretical work could focus on the interface between the absorber and device contact layers, with the aim of minimising the effects of surface recombination. Additionally, it may be beneficial to investigate the solid solution series $\mathrm{Bi}(\mathrm{S}, \mathrm{Se}) \mathrm{I}$ to enable tuning of the electronic, optical, and defect properties for photovoltaic applications.

In the process of performing this work, the field of emerging pho- 
tovoltaics has seen some dramatic changes. The efficiency of perovskite photovoltaics has risen from $\sim 15 \%$ to over $23 \%$. Simultaneously, the development of the layered perovskites as stable alternatives has attracted considerable attention, with most of the best performing perovskite devices now containing a small fraction of the layered analogues. Despite this, the stability of the layered perovskites is not properly understood and future work investigating their tolerance to water is clearly needed. The results presented in this thesis provide an insight into this class of layered materials. Additional work on MAPSI and the Ruddlesden-Popper phases will be necessary to optimise the performance of devices containing these materials. In contrast, while the vacancy-ordered double perovskites initially showed promise due to their ideal optical band gaps, the work in this thesis indicates they posses small fundamental band gaps and are undesirable as solar absorbers. Regardless, they still present interesting toy systems to better understand the ability of iodine anions to provide dispersive conduction band states.

The last few years have also seen the rise of lone-pair materials as promising photovoltaics. These absorbers, containing $\mathrm{Bi}^{3+}$ and $\mathrm{Sb}^{3+}$ cations, are expected to show many similar properties to the hybrid perovskites. Our investigation into one such material, BiSI, reveals it posses optimal optoelectronic properties, but complicated defect behaviour that must be considered during device fabrication. This is in contrast to MAPI, which shows high performance despite relatively high defect concentrations. As such, further work will be necessary to determine whether the defect tuning proposed in this thesis will translate into more efficient devices. 

Appendices 



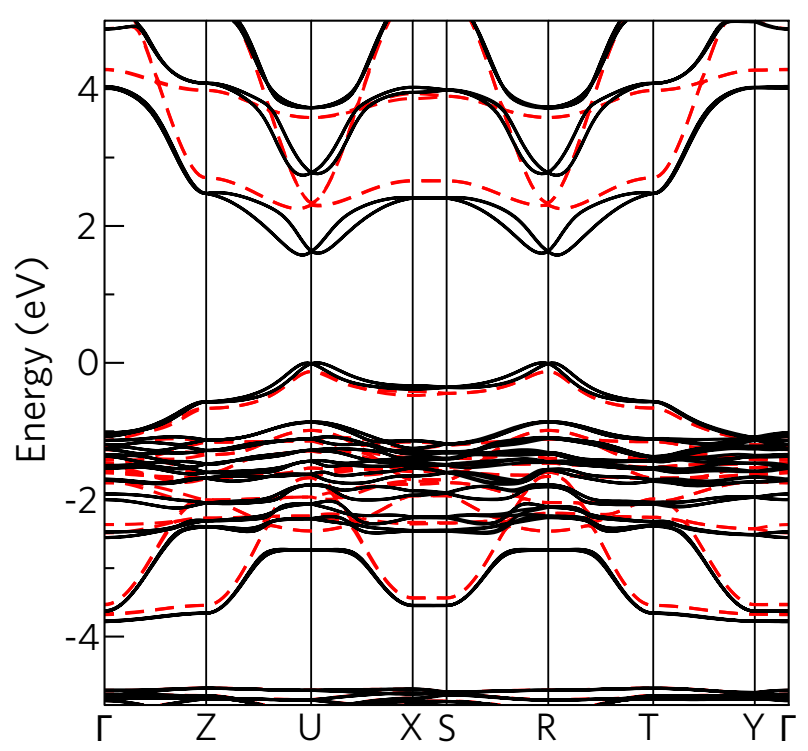

FIGURE A.1: Effect of spin-orbit coupling on the band structure of $\left(\mathrm{CH}_{3} \mathrm{NH}_{3}\right)_{2} \mathrm{~Pb}(\mathrm{SCN})_{2} \mathrm{I}_{2}$. The $\mathrm{HSE}+\mathrm{SOC}$ band structure is shown by black lines, with the HSE only band structure shown via dashed red lines. The valence band maxmimum of the HSE+SOC band structure is set to $0 \mathrm{eV}$. 


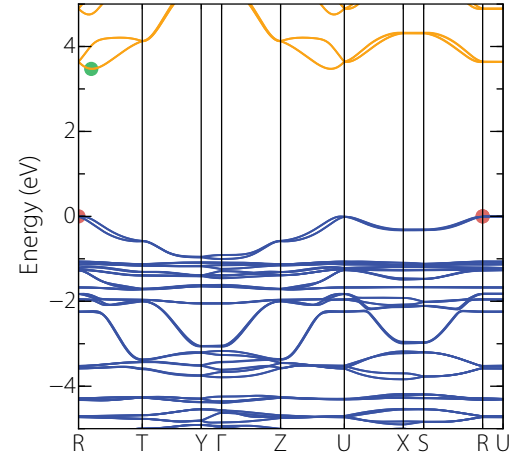

(a)

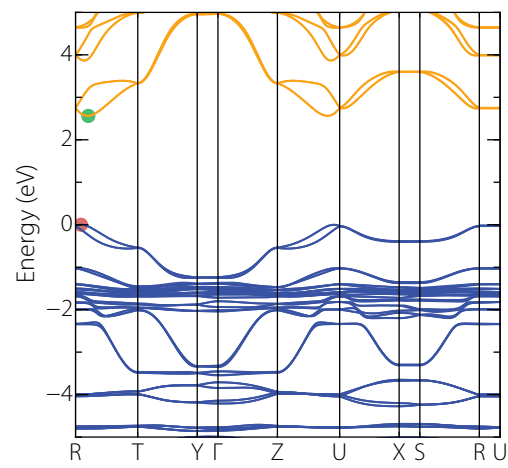

(d)

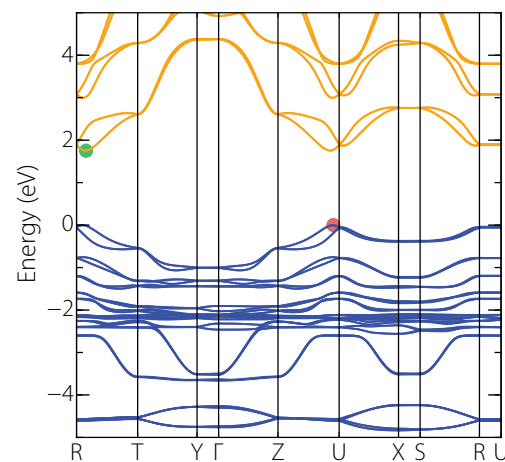

(g)

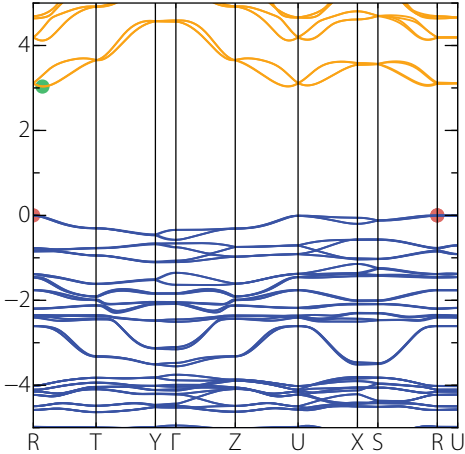

(b)

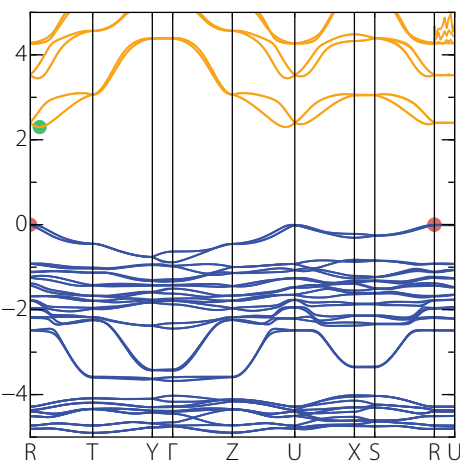

(e)

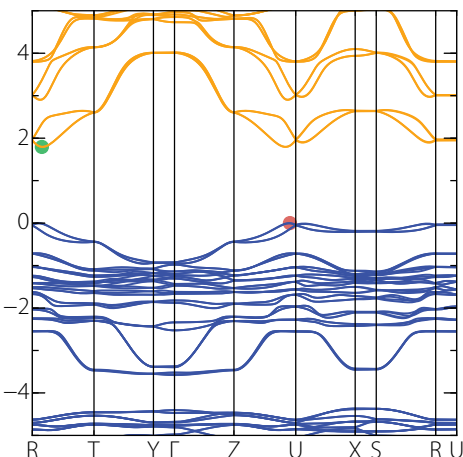

(h)

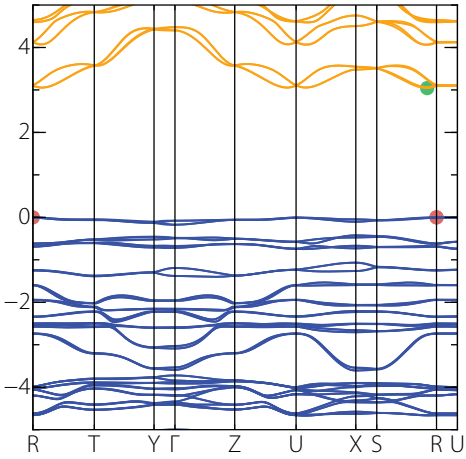

(c)

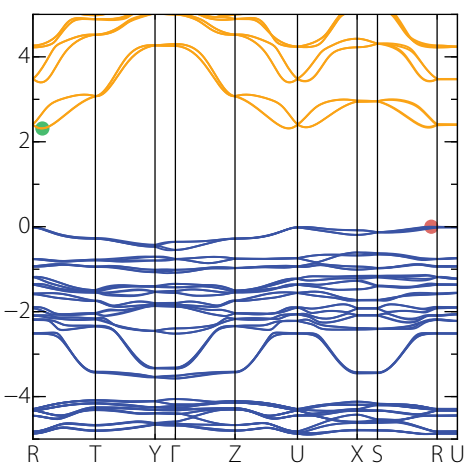

(f)

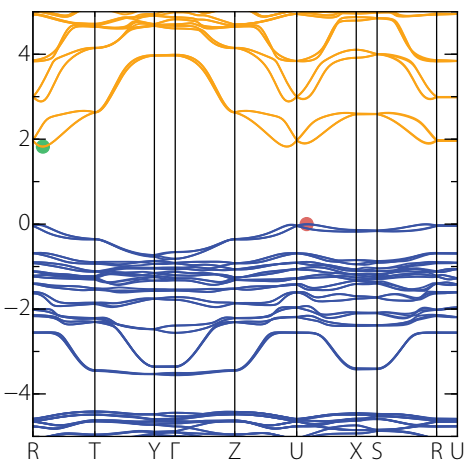

(i)

FIGURE A.2: HSEO6+SOC calculated band structures of lead-based MAPSI-structured analogues: a) $\left(\mathrm{CH}_{3} \mathrm{NH}_{3}\right)_{2} \mathrm{~Pb}(\mathrm{OCN})_{2} \mathrm{Cl}_{2}$, b) $\left.\left(\mathrm{CH}_{3} \mathrm{NH}_{3}\right)_{2} \mathrm{~Pb}(\mathrm{SCN})_{2} \mathrm{Cl}_{2}, \mathrm{c}\right)\left(\mathrm{CH}_{3} \mathrm{NH}_{3}\right)_{2} \mathrm{~Pb}(\mathrm{SeCN})_{2} \mathrm{Cl}_{2}$, d) $\left(\mathrm{CH}_{3} \mathrm{NH}_{3}\right)_{2} \mathrm{~Pb}(\mathrm{OCN})_{2} \mathrm{Br}_{2}$, e) $\left(\mathrm{CH}_{3} \mathrm{NH}_{3}\right)_{2} \mathrm{~Pb}(\mathrm{SCN})_{2} \mathrm{Br}_{2}$, f) $\left(\mathrm{CH}_{3} \mathrm{NH}_{3}\right)_{2} \mathrm{~Pb}(\mathrm{SeCN})_{2} \mathrm{Br}_{2}$, g $)$ $\left(\mathrm{CH}_{3} \mathrm{NH}_{3}\right)_{2} \mathrm{~Pb}(\mathrm{OCN})_{2} \mathrm{I}_{2}$, h) $\left(\mathrm{CH}_{3} \mathrm{NH}_{3}\right)_{2} \mathrm{~Pb}(\mathrm{SCN})_{2} \mathrm{I}_{2}$, i) $\left(\mathrm{CH}_{3} \mathrm{NH}_{3}\right)_{2} \mathrm{~Pb}(\mathrm{SeCN})_{2} \mathrm{I}_{2}$. The valence band maximum is set to $\mathrm{OeV}$. Valence and conduction bands indicated by blue and orange lines, respectively. Green and red circles indicate the valence band maximum and conduction band minimum, respectively. 
$\mathrm{OCN}$

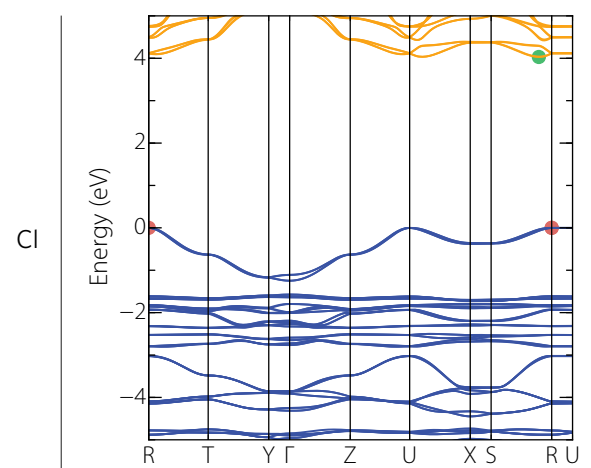

(a)

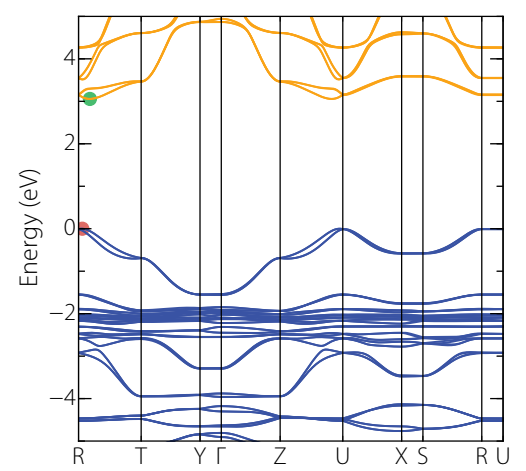

(d)

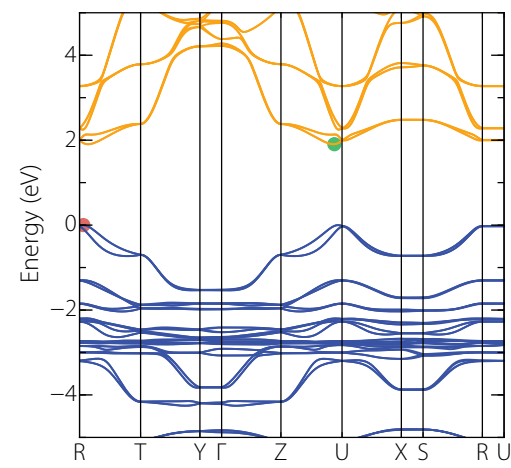

(g)

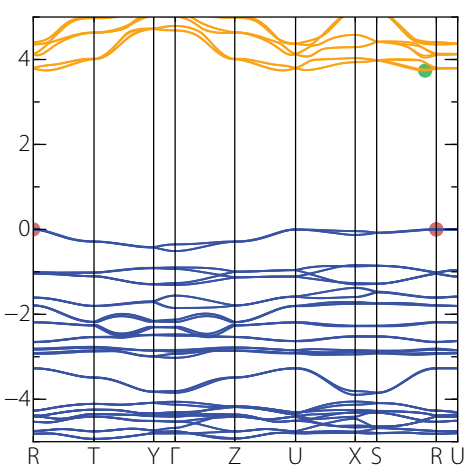

(b)

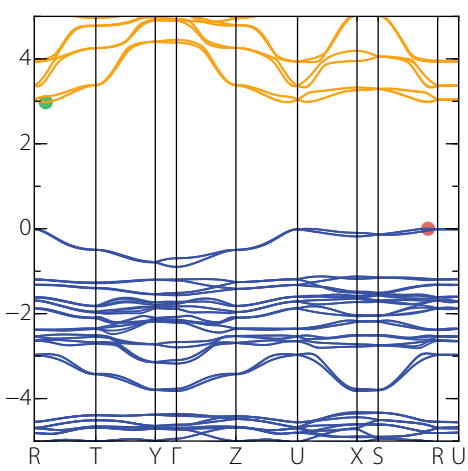

(e)

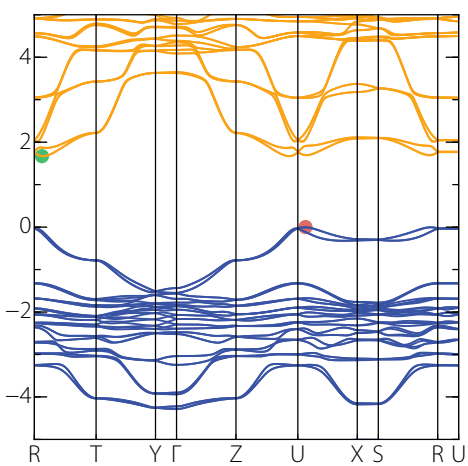

(h)

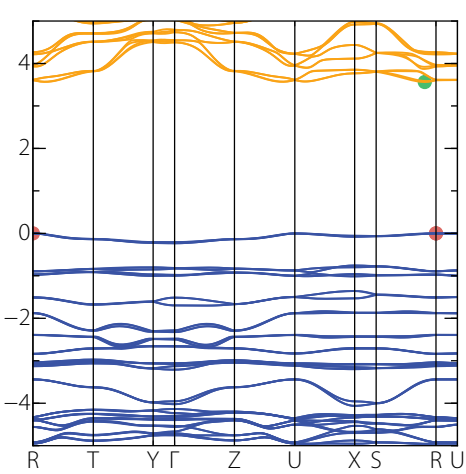

(c)

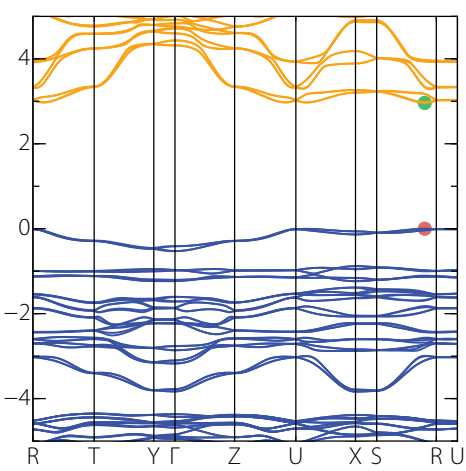

(f)

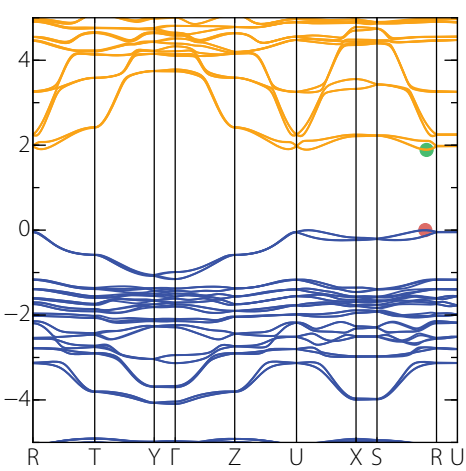

(i)

FIGURE A.3: HSEO6+SOC calculated band structures of tin-based MAPSI-structured analogues: a) $\left(\mathrm{CH}_{3} \mathrm{NH}_{3}\right)_{2} \mathrm{Sn}(\mathrm{OCN})_{2} \mathrm{Cl}_{2}$, b) $\left(\mathrm{CH}_{3} \mathrm{NH}_{3}\right)_{2} \mathrm{Sn}(\mathrm{SCN})_{2} \mathrm{Cl}_{2}$, c) $\left(\mathrm{CH}_{3} \mathrm{NH}_{3}\right)_{2} \mathrm{Sn}(\mathrm{SeCN})_{2} \mathrm{Cl}_{2}$, d) $\left(\mathrm{CH}_{3} \mathrm{NH}_{3}\right)_{2} \mathrm{Sn}(\mathrm{OCN})_{2} \mathrm{Br}_{2}$, e) $\left(\mathrm{CH}_{3} \mathrm{NH}_{3}\right)_{2} \mathrm{Sn}(\mathrm{SCN})_{2} \mathrm{Br}_{2}$, f) $\left(\mathrm{CH}_{3} \mathrm{NH}_{3}\right)_{2} \mathrm{Sn}(\mathrm{SeCN})_{2} \mathrm{Br}_{2}$, g) $\left(\mathrm{CH}_{3} \mathrm{NH}_{3}\right)_{2} \mathrm{Sn}(\mathrm{OCN})_{2} \mathrm{I}_{2}$, h) $\left(\mathrm{CH}_{3} \mathrm{NH}_{3}\right)_{2} \mathrm{Sn}(\mathrm{SCN})_{2} \mathrm{I}_{2}$, i) $\left(\mathrm{CH}_{3} \mathrm{NH}_{3}\right)_{2} \mathrm{Sn}(\mathrm{SeCN})_{2} \mathrm{I}_{2}$. The valence band maximum is set to $\mathrm{OeV}$. Valence and conduction bands indicated by blue and orange lines, respectively. Green and red circles indicate the valence band maximum and conduction band minimum, respectively. 

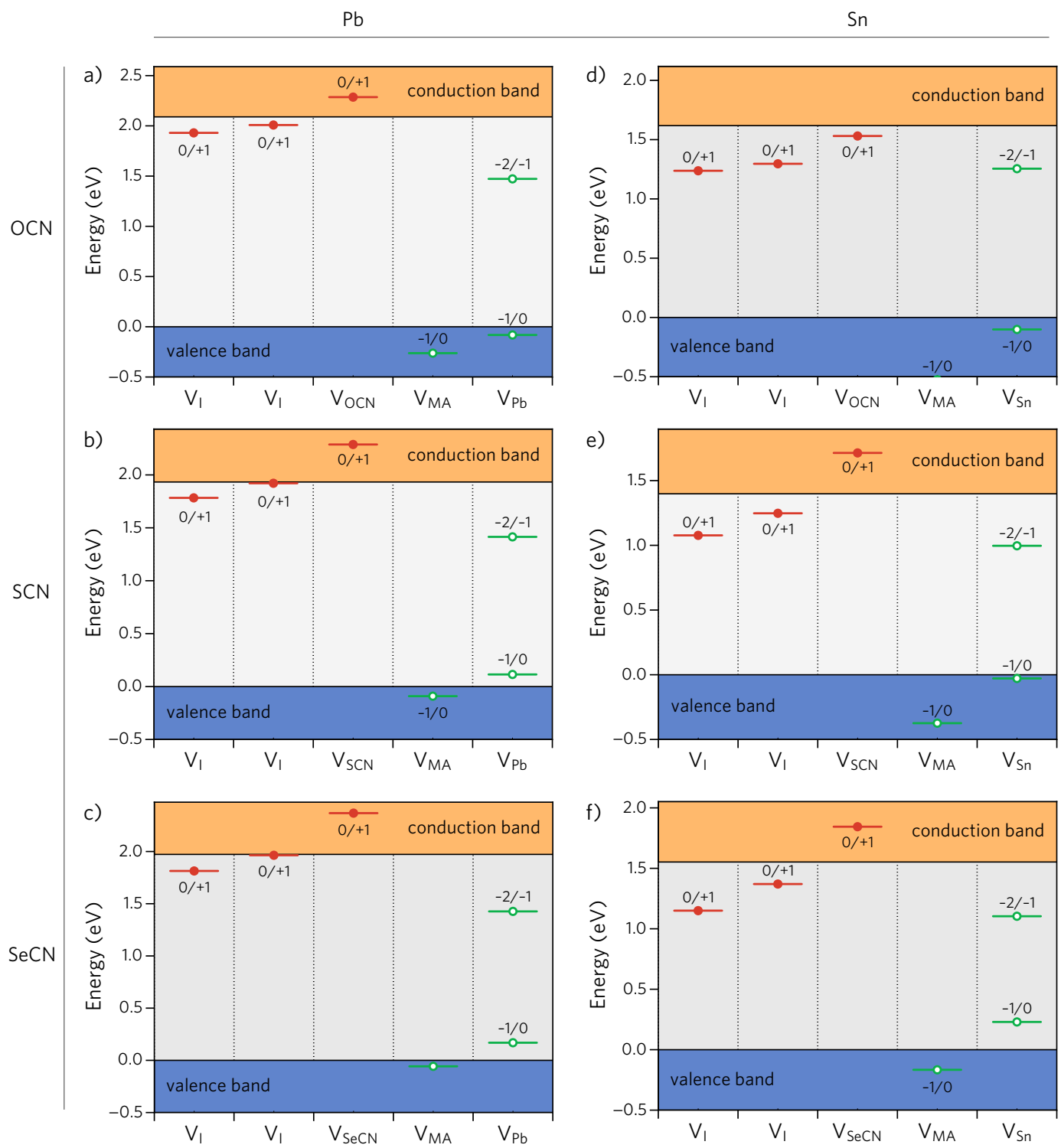

FIGURE A.4: Charge-state transition level diagrams for: a) $\left(\mathrm{CH}_{3} \mathrm{NH}_{3}\right)_{2} \mathrm{~Pb}(\mathrm{OCN})_{2} \mathrm{I}_{2}$, b) $\left(\mathrm{CH}_{3} \mathrm{NH}_{3}\right)_{2} \mathrm{~Pb}(\mathrm{SCN})_{2} \mathrm{I}_{2}$, c) $\left(\mathrm{CH}_{3} \mathrm{NH}_{3}\right)_{2} \mathrm{~Pb}(\mathrm{SeCN})_{2} \mathrm{I}_{2}$, d) $\left(\mathrm{CH}_{3} \mathrm{NH}_{3}\right)_{2} \mathrm{Sn}(\mathrm{OCN})_{2} \mathrm{I}_{2}$, e) $\left.\left(\mathrm{CH}_{3} \mathrm{NH}_{3}\right)_{2} \mathrm{Sn}(\mathrm{SCN})_{2} \mathrm{I}_{2}, \mathrm{f}\right)\left(\mathrm{CH}_{3} \mathrm{NH}_{3}\right)_{2} \mathrm{Sn}(\mathrm{SeCN})_{2} \mathrm{I}_{2}$, The valence band maximum is set to $0 \mathrm{eV}$. Valence and conduction bands indicated by blue and orange lines, respectively. Green and red circles indicate the valence band maximum and conduction band minimum, respectively. Red bands with filled circles indicate donor defects, green bands with open circles indicate acceptor defects. 
TABLE A.1: Relativistic lowering of the indirect $\left(\Delta_{\mathrm{soc}} E_{\mathrm{g}}^{\mathrm{ind}}\right)$ and direct $\left(\Delta_{\mathrm{soc}} E_{\mathrm{g}}^{\mathrm{dir}}\right)$ band gaps of $\left(\mathrm{CH}_{3} \mathrm{NH}_{3}\right)_{2} \mathrm{MPs}_{2} \mathrm{I}_{2}$, where $\mathrm{M}=\mathrm{Sn}, \mathrm{Pb}$, and $\mathrm{Ps}=\mathrm{OCN}, \mathrm{SCN}$, SeCN, calculated using HSEO6 with and without spin-orbit coupling (SOC))

\begin{tabular}{|c|c|c|}
\hline Compound & $\Delta_{\text {soc }} E_{\mathrm{g}}^{\text {ind }}(\mathrm{eV})$ & $\Delta_{\text {soc }} E_{\mathrm{g}}^{\mathrm{dir}}(\mathrm{eV})$ \\
\hline $\mathrm{MA}_{2} \mathrm{~Pb}(\mathrm{OCN})_{2} \mathrm{Cl}_{2}$ & 0.75 & 0.82 \\
\hline $\mathrm{MA}_{2} \mathrm{~Pb}(\mathrm{SCN})_{2} \mathrm{Cl}_{2}$ & 0.63 & 0.65 \\
\hline $\mathrm{MA}_{2} \mathrm{~Pb}(\mathrm{SeCN})_{2} \mathrm{Cl}_{2}$ & 0.60 & 0.60 \\
\hline $\mathrm{MA}_{2} \mathrm{~Pb}(\mathrm{OCN})_{2} \mathrm{Br}_{2}$ & 0.81 & 0.88 \\
\hline $\mathrm{MA}_{2} \mathrm{~Pb}(\mathrm{SeCN})_{2} \mathrm{Br}_{2}$ & 0.70 & 0.73 \\
\hline $\mathrm{MA}_{2} \mathrm{~Pb}(\mathrm{OCN})_{2} \mathrm{I}_{2}$ & 0.87 & 0.93 \\
\hline $\mathrm{MA}_{2} \mathrm{~Pb}(\mathrm{SCN})_{2} \mathrm{I}_{2}$ & 0.77 & 0.78 \\
\hline $\mathrm{MA}_{2} \mathrm{~Pb}(\mathrm{SeCN})_{2} \mathrm{I}_{2}$ & 0.73 & 0.76 \\
\hline $\mathrm{MA}_{2} \mathrm{Sn}(\mathrm{OCN})_{2} \mathrm{Cl}_{2}$ & 0.16 & 0.15 \\
\hline $\mathrm{MA}_{2} \mathrm{Sn}(\mathrm{SCN})_{2} \mathrm{Cl}_{2}$ & 0.11 & 0.12 \\
\hline $\mathrm{MA}_{2} \mathrm{Sn}(\mathrm{SeCN})_{2} \mathrm{Cl}_{2}$ & 0.11 & 0.12 \\
\hline $\mathrm{MA}_{2} \mathrm{Sn}(\mathrm{OCN})_{2} \mathrm{Br}_{2}$ & 0.16 & 0.16 \\
\hline $\mathrm{MA}_{2} \mathrm{Sn}(\mathrm{SCN})_{2} \mathrm{Br}_{2}$ & 0.12 & 0.14 \\
\hline $\mathrm{MA}_{2} \mathrm{Sn}(\mathrm{SeCN})_{2} \mathrm{Br}_{2}$ & 0.12 & 0.14 \\
\hline $\mathrm{MA}_{2} \mathrm{Sn}(\mathrm{OCN})_{2} \mathrm{I}_{2}$ & 0.22 & 0.25 \\
\hline $\mathrm{MA}_{2} \mathrm{Sn}(\mathrm{SCN})_{2} \mathrm{I}_{2}$ & 0.20 & 0.23 \\
\hline $\mathrm{MA}_{2} \mathrm{Sn}(\mathrm{SeCN})_{2} \mathrm{I}_{2}$ & 0.20 & 0.22 \\
\hline
\end{tabular}




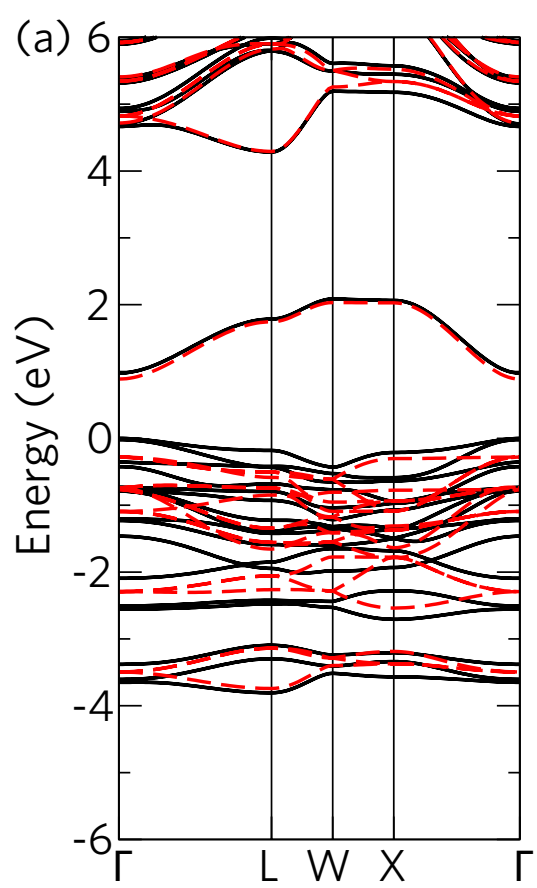

(b)

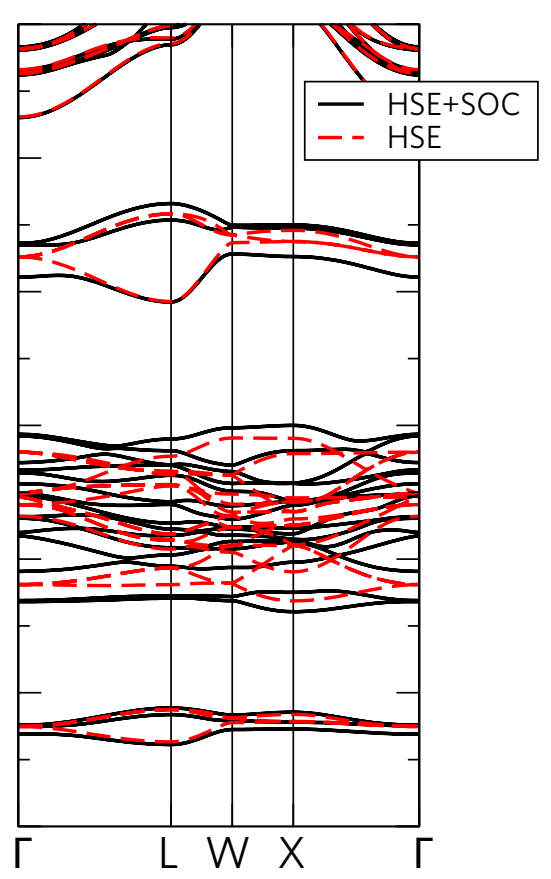

FIGURE B.1: Effect of spin-orbit coupling on the band structures of (a) $\mathrm{Cs}_{2} \mathrm{SnI}_{6}$ and (b) $\mathrm{Cs}_{2} \mathrm{Tel}_{6}$. The $\mathrm{HSE}+\mathrm{SOC}$ band structure is shown by black lines, with the HSE only band structure shown via dashed red lines. The valence band maxmimum of the $\mathrm{HSE}+\mathrm{SOC}$ band structure is set to $0 \mathrm{eV}$. 


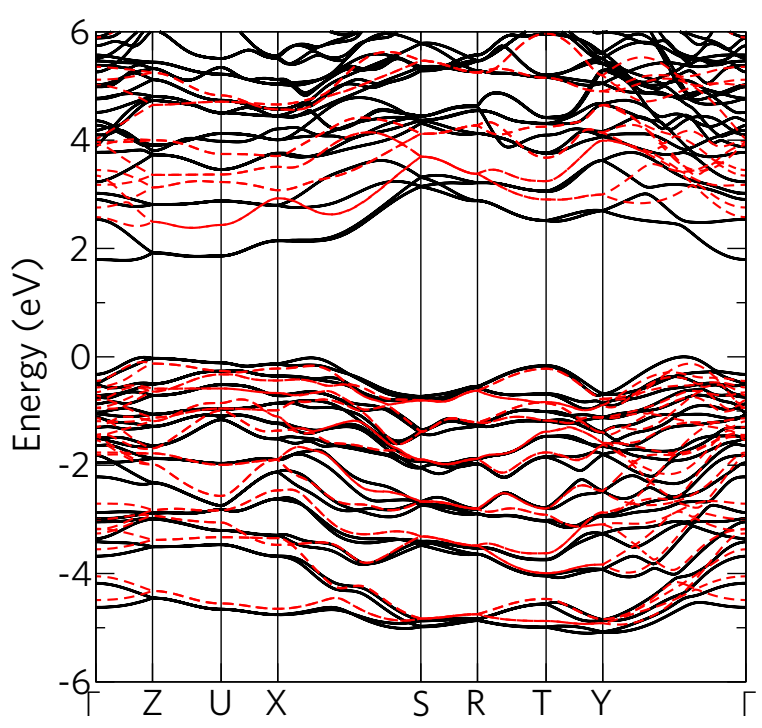

(a)

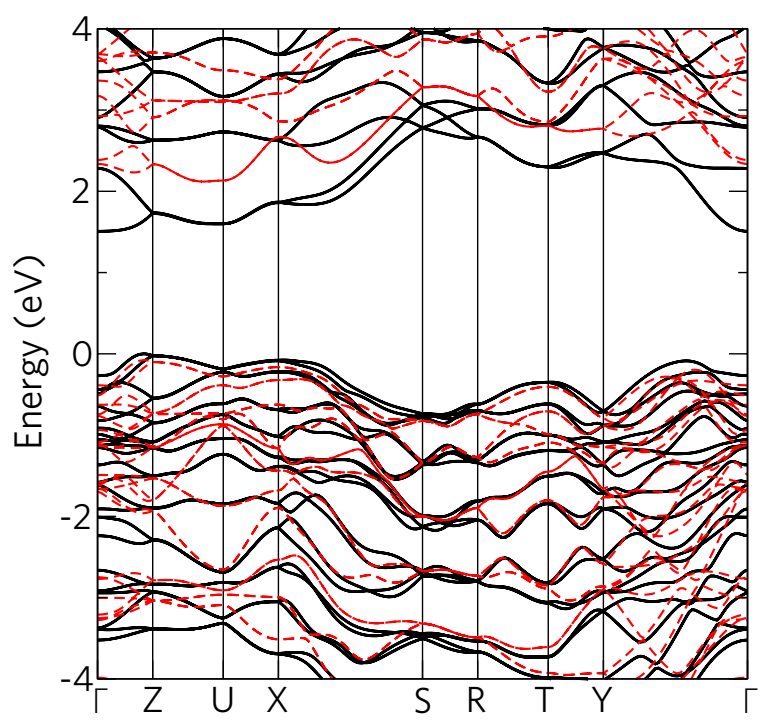

(b)

FIGURE C.1: Effect of spin-orbit coupling on the band structures of (a) BiSI and (b) BiSel. The $\mathrm{HSE}+\mathrm{SOC}$ band structure is shown by black lines, with the HSE only band structure shown via dashed red lines. The valence band maxmimum of the $\mathrm{HSE}+\mathrm{SOC}$ band structure is set to $0 \mathrm{eV}$. 

Indexes and bibliography 



\section{LIST OF FIGURES}

1.1 Simplified representation of a $p-n$ junction $\ldots \ldots \ldots \ldots$

1.2 Schematic of a $p-n$ junction solar cell $\ldots \ldots \ldots \ldots$

1.3 Example $J-V$ curve for a solar cell under illumination $\ldots \ldots \ldots \ldots$

1.4 Example plot of external quantum efficiency against photon wavelength . . . . 8

1.5 Common electron-hole recombination mechanisms in photovoltaic devices . . 9

1.6 Optimal range of photovoltaic absorber band gaps . . . . . . . . . . . . 10

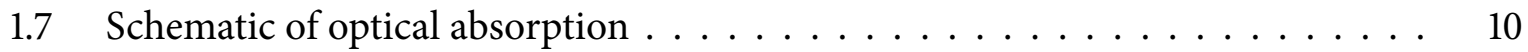

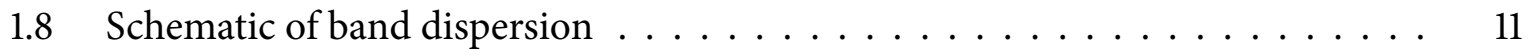

1.9 Importance of bonding structure on defect tolerance $\ldots \ldots \ldots \ldots \ldots$

1.10 Absorption and recombination in Rashba and Dresselhaus spin-split systems . . 12

1.11 Schematic of band alignment in a heterojunction solar cell . . . . . . . . . . 12

1.12 Comparison of elemental cost and abundance in the earth's crust . . . . . . . . 13

1.13 Efficiency improvements for several photovoltaic absorbers . . . . . . . . . 15

2.1 Representation of primitive and conventional cells . . . . . . . . . . . . 27

2.2 Comparison of all electron and pseudopotential wavefunction and potential . . 30

3.1 Example transition level diagrams $\ldots \ldots \ldots \ldots \ldots$

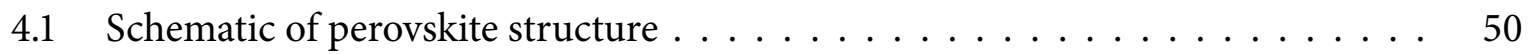

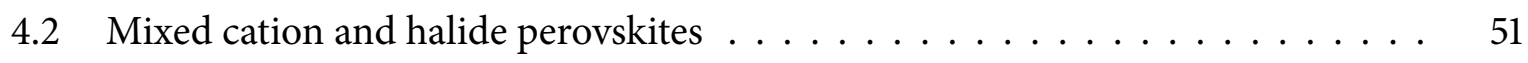

4.3 Relationship between three-dimensional and layered perovskites . . . . . . . 53

$4.4 J-V$ curves for pyrazine containing $\mathrm{FASnI}_{3}$ devices $\ldots \ldots \ldots \ldots$

5.1 Crystal structure of $\left(\mathrm{CH}_{3} \mathrm{NH}_{3}\right)_{2} \mathrm{~Pb}(\mathrm{SCN})_{2} \mathrm{I}_{2} \ldots \ldots \ldots \ldots \ldots \ldots \ldots$

5.2 Brillouin zone of the $P n m 2_{1}$ space group $\ldots \ldots \ldots \ldots \ldots \ldots \ldots$

5.3 Electronic structures of $\left(\mathrm{CH}_{3} \mathrm{NH}_{3}\right)_{2} \mathrm{~Pb}(\mathrm{SCN})_{2} \mathrm{I}_{2}$ and $\mathrm{CH}_{3} \mathrm{NH}_{3} \mathrm{PbI}_{3} \ldots \ldots . \ldots 3$

5.4 Charge density isosurfaces of $\left(\mathrm{CH}_{3} \mathrm{NH}_{3}\right)_{2} \mathrm{~Pb}(\mathrm{SCN})_{2} \mathrm{I}_{2} \ldots \ldots \ldots \ldots$

5.5 Charge-state transition level diagram for $\left(\mathrm{CH}_{3} \mathrm{NH}_{3}\right)_{2} \mathrm{~Pb}(\mathrm{SCN})_{2} \mathrm{I}_{2} \ldots \ldots \ldots$

5.6 Band structure of MAPSI-structured cyanate compounds . . . . . . . . 71

5.7 Band structure of MAPSI-structured bromide and iodide compounds . . . . . 72

5.8 Density of states for MAPSI-structured analogues . . . . . . . . . . . 73

5.9 Band alignments of MAPSI-structured analogues . . . . . . . . . . . . 75 
5.10 Charge-state transition level diagrams for MAPSI analogues . . . . . . . . . 77

6.1 Crystal structure of $\mathrm{Cs}_{2} \mathrm{SnI}_{6} \ldots \ldots \ldots \ldots \ldots \ldots \ldots \ldots$

6.2 Structural data for the solid-solution series, $\mathrm{Cs}_{2} \mathrm{Sn}_{1-x} \mathrm{Te}_{x} \mathrm{I}_{6} \ldots \ldots \ldots \ldots$

6.3 Optoelectronic properties for the series $\mathrm{Cs}_{2} \mathrm{Sn}_{1-x} \mathrm{Te}_{x} \mathrm{I}_{6} \ldots \ldots \ldots \ldots \ldots$

6.4 Brillouin zone of the $F m \overline{3} m$ space group . . . . . . . . . . . . 83

6.5 Orbital projected band structures of $\mathrm{Cs}_{2} \mathrm{SnI}_{6}$ and $\mathrm{Cs}_{2} \mathrm{TeI}_{6} \ldots \ldots \ldots \ldots$

6.6 Crystal orbital Hamilton population analysis of $\mathrm{Cs}_{2} \mathrm{SnI}_{6}$ and $\mathrm{Cs}_{2} \mathrm{TeI}_{6} \ldots \ldots$. . . 86

6.7 Simplified molecular orbital picture of $\mathrm{CsSnI}_{3}, \mathrm{Cs}_{2} \mathrm{SnI}_{6}$, and $\mathrm{Cs}_{2} \mathrm{TeI}_{6} \ldots \ldots$

6.8 Band structure of $\mathrm{Cs}_{2} \mathrm{SnI}_{6} \ldots \ldots \ldots \ldots \ldots \ldots \ldots$

6.9 Analysis of antibonding states in $\mathrm{Cs}_{2} \mathrm{SnI}_{6} \ldots \ldots \ldots \ldots \ldots \ldots$

6.10 Analysis of bonding states in $\mathrm{Cs}_{2} \mathrm{SnI}_{6} \ldots \ldots \ldots \ldots \ldots$

6.11 Band alignment of $\mathrm{Cs}_{2} \mathrm{SnI}_{6}$ and $\mathrm{Cs}_{2} \mathrm{TeI}_{6} \ldots \ldots \ldots \ldots \ldots$

6.12 Accessible chemical potential ranges of $\mathrm{Cs}_{2} \mathrm{SnI}_{6}$ and $\mathrm{Cs}_{2} \mathrm{TeI}_{6} \ldots \ldots \ldots$

6.13 Defect formation energies for iodine vacancies in $\mathrm{Cs}_{2} \mathrm{SnI}_{6}$ and $\mathrm{Cs}_{2} \mathrm{TeI}_{6} \ldots \ldots$

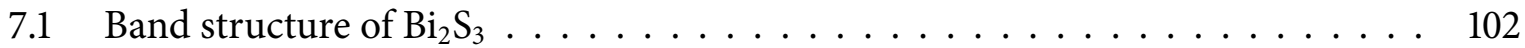

7.2 Crystal structure and band structure of $\mathrm{BiI}_{3} \ldots \ldots \ldots \ldots \ldots \ldots \ldots \ldots \ldots$

7.3 Crystal structure and band structure of $\mathrm{Rb}_{3} \mathrm{Bi}_{2} \mathrm{I}_{9}$ and $\mathrm{Cs}_{3} \mathrm{Bi}_{2} \mathrm{I}_{9} \ldots \ldots \ldots \ldots$

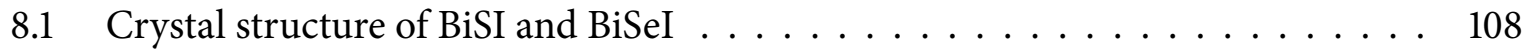

8.2 Brillouin zone of the Pnma space group . . . . . . . . . . . . . . . . . . . 109

8.3 Band structure and density of states of BiSI and BiSeI . . . . . . . . . . . 111

8.4 Crystal orbital Hamilton population analysis of BiSI and BiSeI . . . . . . 113

8.5 Charge density isosurfaces of BiSeI . . . . . . . . . . . . . . . . . . 114

8.6 Band alignment of BiSI and BiSeI . . . . . . . . . . . . . . 116

8.7 Accessible chemical potentials for BiSI and BiSeI . . . . . . . . . . . . 117

8.8 Interstitial sites in BiSI crystal structure . . . . . . . . . . . . . . 118

8.9 Defect formation energies of BiSI under $p$-type conditions . . . . . . . . . 118

8.10 Defect formation energies of BiSI under $n$-type conditions . . . . . . . . . . . 119

8.11 Charge densities of charged sulfur vacancy defects in BiSI . . . . . . . . . 120

8.12 Defect formation energies of BiSeI under $p$-type conditions $\ldots \ldots \ldots \ldots \ldots$

8.13 Defect formation energies of BiSeI under $n$-type conditions . . . . . . . . . 122

8.14 Shockley-Read-Hall recombination in BiSI and BiSeI . . . . . . . . . . . . 124

A.1 Effect of spin-orbit coupling on $\left(\mathrm{CH}_{3} \mathrm{NH}_{3}\right)_{2} \mathrm{~Pb}(\mathrm{SCN})_{2} \mathrm{I}_{2} \ldots \ldots \ldots \ldots$ 
A.2 Band structures of lead-based MAPSI-structured analogues $\ldots \ldots \ldots \ldots \ldots$

A.3 Band structures of tin-based MAPSI-structured analogues . . . . . . . . . 136

A.4 Charge-state transition level diagrams for MAPSI-structured analogues . . . . 137

B.1 Effect of spin-orbit coupling on $\mathrm{Cs}_{2} \mathrm{SnI}_{6}$ and $\mathrm{Cs}_{2} \mathrm{TeI}_{6} \ldots \ldots \ldots \ldots \ldots$

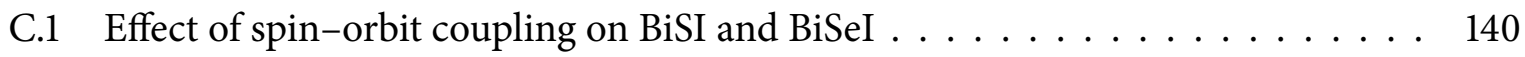




\section{BIBLIOGRAPHY}

[1] Khan, M. A.; Khan, M. Z.; Zaman, K.; Naz, L. Global estimates of energy consumption and greenhouse gas emissions. Renewable Sustainable Energy Rev. 2014, 29, 336-344.

[2] Martens, P. Health and climate change: modelling the impacts of global warming and ozone depletion; Routledge, 2014.

[3] World Health Organization, 7 million premature deaths annually linked to air pollution. 2014; http://www. who.int/phe/health_topics/outdoorair/databases/FINAL_ HAP_AAP_BoD_24March2014.pdf.

[4] Jäger-Waldau, A. Photovoltaics and renewable energies in Europe. Renew. Sust. Energ. Rev. 2007, 11, 1414-1437.

[5] Lewis, N. S.; Nocera, D. G. Powering the planet: Chemical challenges in solar energy utilization. Proc. Natl. Acad. Sci. 2006, 103, 15729-15735.

[6] Hermann, W. A. Quantifying global exergy resources. Energy 2006, 31, 1685-1702.

[7] Nelson, J. The physics of solar cells; World Scientific, 2003; Vol. 57.

[8] Ondraczek, J.; Komendantova, N.; Patt, A. WACC the Dog: The effect of financing costs on the levelized cost of solar PV power. Renew. Energy 2015, 75, 888-898.

[9] Brandt, R. E.; Stevanović, V.; Ginley, D. S.; Buonassisi, T. Identifying Defect-Tolerant Semiconductors with High Minority-Carrier Lifetimes: Beyond Hybrid Lead Halide Perovskites. MRS Commun. 2015, 5, 1-11.

[10] Shockley, W.; Queisser, H. J. Detailed Balance Limit of Efficiency of $p-n$ Junction Solar Cells. J. Appl. Phys. 1961, 32, 510-519.

[11] De Wolf, S.; Holovsky, J.; Moon, S.-J.; Löper, P.; Niesen, B.; Ledinsky, M.; Haug, F.-J.; Yum, J.-H.; Ballif, C. Organometallic halide perovskites: sharp optical absorption edge and its relation to photovoltaic performance. J. Phys. Chem. Lett. 2014, 5, 1035-1039.

[12] Yu, L.; Zunger, A. Identification of Potential Photovoltaic Absorbers Based on FirstPrinciples Spectroscopic Screening of Materials. Phys. Rev. Lett. 2012, 108, 068701. 
[13] Kehoe, A. B.; Scanlon, D. O.; Watson, G. W. Nature of the band gap of $\mathrm{Tl}_{2} \mathrm{O}_{3}$. Phys. Rev. B 2011, 83, 3-6.

[14] Ganose, A. M.; Scanlon, D. O. Band gap and work function tailoring of $\mathrm{SnO}_{2}$ for improved transparent conducting ability in photovoltaics. J. Mater. Chem. C 2016, 4, 1467-1475.

[15] Mattheis, J.; Werner, J. H.; Rau, U. Finite mobility effects on the radiative efficiency limit of $p-n$ junction solar cells. Phys. Rev. B 2008, 77, 085203.

[16] Jaramillo, R.; Sher, M.-J.; Ofori-Okai, B. K.; Steinmann, V.; Yang, C.; Hartman, K.; Nelson, K. A.; Lindenberg, A. M.; Gordon, R. G.; Buonassisi, T. Transient terahertz photoconductivity measurements of minority-carrier lifetime in tin sulfide thin films: Advanced metrology for an early stage photovoltaic material. J. Appl. Phys. 2016, 119, 035101.

[17] Maughan, A. E.; Ganose, A. M.; Bordelon, M. M.; Miller, E. M.; Scanlon, D. O.; Neilson, J. R. Defect tolerance to intolerance in the vacancy-ordered double perovskite semiconductors $\mathrm{Cs}_{2} \mathrm{SnI}_{6}$ and $\mathrm{Cs}_{2} \mathrm{TeI}_{6}$. J. Am. Chem. Soc. 2016, 138, 8453-8464.

[18] Zakutayev, A.; Caskey, C. M.; Fioretti, A. N.; Ginley, D. S.; Vidal, J.; Stevanovic, V.; Tea, E.; Lany, S. Defect Tolerant Semiconductors for Solar Energy Conversion. J. Phys. Chem. Lett. 2014, 5, 1117-1125.

[19] Bube, R. H. Photoelectronic Properties of Semiconductors; Cambridge University Press, 1992.

[20] Kutes, Y.; Ye, L.; Zhou, Y.; Pang, S.; Huey, B. D.; Padtures, N. P. Direct Observation of Ferroelectric Domains in Solution-Processed $\mathrm{CH}_{3} \mathrm{NH}_{3} \mathrm{PbI}_{3}$ Perovskite Thin Films. J. Phys. Chem. Lett. 2014, 5, 3335-3339.

[21] Liu, S.; Zheng, F.; Koocher, N. Z.; Takenaka, H.; Wang, F.; Rappe, A. M. Ferroelectric Domain Wall Induced Band Gap Reduction and Charge Separation in Organometal Halide Perovskites. J. Phys. Chem. Lett. 2015, 6, 693-699.

[22] Frost, J. M.; Butler, K. T.; Brivio, F; Hendon, C. H.; Van Schilfgaarde, M.; Walsh, A. Atomistic Origins of High-Performance in Hybrid Halide Perovskite Solar Cells. Nano Lett 2014, 14, 2584-2590.

[23] Butler, K. T.; Frost, J. M.; Walsh, A. Band Alignment of the Hybrid Halide Perovskites $\mathrm{CH}_{3} \mathrm{NH}_{3} \mathrm{PbCl}_{3}, \mathrm{CH}_{3} \mathrm{NH}_{3} \mathrm{PbBr}_{3}$ and $\mathrm{CH}_{3} \mathrm{NH}_{3} \mathrm{PbI}_{3}$. Mater. Horiz. 2015, 2, 228-231. 
[24] Shao, Y.; Xiao, Z.; Bi, C.; Yuan, Y.; Huang, J. Origin and Elimination of Photocurrent Hysteresis by Fullerene Passivation in $\mathrm{CH}_{3} \mathrm{NH}_{3} \mathrm{PbI}_{3}$ Planar Heterojunction Solar Cells. Nat. Commun. 2014, 5, 5784.

[25] Kepenekian, M.; Robles, R.; Katan, C.; Sapori, D.; Pedesseau, L.; Even, J. Rashba and Dresselhaus Effects in Hybrid Organic-Inorganic Perovskites: From Basics to Devices. ACS Nano 2015, 9, 11557-11567.

[26] Azarhoosh, P.; Frost, J. M.; McKechnie, S.; Walsh, A.; van Schilfgaarde, M. Relativistic Origin of slow electron-hole recombination in hybrid halide perovskite solar cells. APL Mater. 2016, 4, 091501.

[27] Hutter, E. M.; Gélvez-Rueda, M. C.; Osherov, A.; Bulović, V.; Grozema, F. C.; Stranks, S. D.; Savenije, T. J. Direct-indirect character of the bandgap in methylammonium lead iodide perovskite. Nat. Mater. 2016, 16, In press.

[28] Etienne, T.; Mosconi, E.; Angelis, F. D. Dynamical Origin of the Rashba Effect in Organohalide Lead Perovskites: A Key to Suppressed Carrier Recombination in Perovskite Solar Cells? J. Phys. Chem. Lett. 2016, 7, 1638-1645.

[29] Yoshikawa, K.; Kawasaki, H.; Yoshida, W.; Irie, T.; Konishi, K.; Nakano, K.; Uto, T.; Adachi, D.; Kanematsu, M.; Uzu, H.; Yamamoto, K. Silicon heterojunction solar cell with interdigitated back contacts for a photoconversion efficiency over 26\%. Nature Energy 2017, 2, 17032.

[30] Peng, J.; Lu, L.; Yang, H. Review On Life Cycle Assessment of Energy Payback and Greenhouse Gas Emission of Solar Photovoltaic Systems. Renew. Sust. Energ. Rev. 2013, 19, 255274.

[31] Lacerda, J. S.; van den Bergh, J. C. Diversity in solar photovoltaic energy: Implications for innovation and policy. Renewable Sustainable Energy Rev. 2016, 54, 331-340.

[32] Rubin, E. S.; Azevedo, I. M.; Jaramillo, P.; Yeh, S. A review of learning rates for electricity supply technologies. Energy Policy 2015, 86, 198-218.

[33] Wadia, C.; Alivisatos, A. P.; Kammen, D. M. Materials availability expands the opportunity for large-scale photovoltaics deployment. Environ. Sci. Technol. 2009, 43, 2072-2077.

[34] Dhere, N. G. Toward GW/year of CIGS production within the next decade. Sol. Energy Mater. Sol. Cells 2007, 91, 1376-1382. 
[35] Green, M. A.; Ho-Baillie, A.; Snaith, H. J. The Emergence of Perovskite Solar Cells. Nat. Photon. 2014, 8, 506-514.

[36] Grätzel, M. The Light and Shade of Perovskite Solar Cells. Nat. Mater. 2014, 13, 838-842.

[37] A., G. M.; Yoshihiro, H.; D., D. E.; H., L. D.; Jochen, H.-E.; W.Y., H.-B. A. Solar cell efficiency tables (version 51). Prog. Photovoltaics Res. Appl. 2017, 26, 3-12.

[38] Baikie, T.; Fang, Y.; Kadro, J. M.; Schreyer, M.; Wei, F.; Mhaisalkar, S. G.; Graetzel, M.; White, T. J. Synthesis and Crystal Chemistry of the Hybrid Perovskite $\left(\mathrm{CH}_{3} \mathrm{NH}_{3}\right) \mathrm{PbI}_{3}$ for Solid-State Sensitised Solar Cell Applications. J. Mater. Chem. A 2013, 1, 5628-5641.

[39] D’Innocenzo, V.; Grancini, G.; Alcocer, M. J.; Kandada, A. R. S.; Stranks, S. D.; Lee, M. M.; Lanzani, G.; Snaith, H. J.; Petrozza, A. Excitons Versus Free Charges in Organo-Lead TriHalide Perovskites. Nat. Commun. 2014, 5, 3586.

[40] Walsh, A.; Scanlon, D. O.; Chen, S.; Gong, X.; Wei, S.-H. Self-Regulation Mechanism for Charged Point Defects in Hybrid Halide Perovskites. Angew. Chem. 2015, 127, 1811-1814.

[41] Deng, Y.; Peng, E.; Shao, Y.; Xiao, Z.; Dong, Q.; Huang, J. Scalable Fabrication of Efficient Organolead Trihalide Perovskite Solar Cells with Doctor-Bladed Active Layers. Energy Environ. Sci. 2015, 8, 1544-1550.

[42] Xing, G.; Mathews, N.; Sun, S.; Lim, S. S.; Lam, Y. M.; Gratzel, M.; Mhaisalkar, S.; Sum, T. C. Long-Range Balanced Electron- and Hole-Transport Lengths in OrganicInorganic $\mathrm{CH}_{3} \mathrm{NH}_{3} \mathrm{PbI}_{3}$. Science 2013, 342, 344-347.

[43] Wehrenfennig, C.; Eperon, G. E.; Johnston, M. B.; Snaith, H. J.; Herz, L. M. High Charge Carrier Mobilities and Lifetimes in Organolead Trihalide Perovskites. Adv. Mater. 2014, $26,1584-1589$.

[44] Zhao, Y.; Zhu, K. Charge Transport and Recombination in Perovskite $\left(\mathrm{CH}_{3} \mathrm{NH}_{3}\right) \mathrm{PbI}_{3}$ Sensitized $\mathrm{TiO}_{2}$ Solar Cells. J. Phys. Chem. Lett. 2013, 4, 2880-2884.

[45] Barrows, A. T.; Pearson, A. J.; Kwak, C. K.; Dunbar, A. D. F.; Buckley, A. R.; Lidzey, D. G. Efficient Planar Heterojunction Mixed-halide Perovskite Solar Cells Deposited Via SprayDeposition. Energy Environ. Sci. 2014, 7, 2944. 
[46] Bhachu, D. S.; Scanlon, D. O.; Saban, E. J.; Bronstein, H.; Parkin, I. P.; Carmalt, C. J.; Palgrave, R. G. Scalable Route to $\mathrm{CH}_{3} \mathrm{NH}_{3} \mathrm{PbI}_{3}$ Perovskite Thin Films by Aerosol Assisted Chemical Vapour Deposition. J. Mater. Chem. A 2015, 3, 9071-9073.

[47] Hodes, G.; Cahen, D. Photovoltaics Perovskite Cells Roll Forward. Nat. Photon. 2014, 8, $87-88$.

[48] Christians, J. A.; Manser, J. S.; Kamat, P. V. Best Practices in Perovskite Solar Cell Efficiency Measurements. Avoiding the Error of Making Bad Cells Look Good. J. Phys. Chem. Lett. $2015,6,852-857$.

[49] Guarnera, S.; Abate, A.; Zhang, W.; Foster, J. M.; Richardson, G.; Petrozza, A.; Snaith, H. J. Improving the Long-Term Stability of Perovskite Solar Cells with a Porous $\mathrm{Al}_{2} \mathrm{O}_{3}$ Buffer Layer. J. Phys. Chem. Lett. 2015, 6, 432-437.

[50] Niu, G.; Guo, X.; Wang, L. Review of Recent Progress in Chemical Stability of Perovskite Solar Cells. J. Mater. Chem. A 2015, 3, 8970-8980.

[51] Schrödinger, E. An undulatory theory of the mechanics of atoms and molecules. Phys. Rev. $1926,28,1049$.

[52] Schrödinger, E. Quantisierung als eigenwertproblem. Annalen der physik 1926, 385, 437490.

[53] Fock, V. Näherungsmethode zur Lösung des quantenmechanischen Mehrkörperproblems. Zeitschrift für Physik 1930, 61, 126-148.

[54] Kohn, W. Nobel Lecture: Electronic structure of matter-wave functions and density functionals. Rev. Mod. Phys. 1999, 71, 1253.

[55] Slater, J. C. The theory of complex spectra. Phys. Rev. 1929, 34, 1293.

[56] Löwdin, P.-O. Quantum theory of many-particle systems. I. Physical interpretations by means of density matrices, natural spin-orbitals, and convergence problems in the method of configurational interaction. Phys. Rev. 1955, 97, 1474.

[57] Hohenberg, P.; Kohn, W. Inhomogeneous electron gas. Phys. Rev. 1964, 136, B864.

[58] Bloch, F. Über die quantenmechanik der elektronen in kristallgittern. Zeitschrift für physik $1929,52,555-600$. 
[59] Dirac, P. A. Note on exchange phenomena in the Thomas atom. Mathematical Proceedings of the Cambridge Philosophical Society. 1930; pp 376-385.

[60] Ceperley, D. M.; Alder, B. Ground state of the electron gas by a stochastic method. Phys. Rev. Lett. 1980, 45, 566.

[61] Vosko, S.; Wilk, L.; Nusair, M. Accurate spin-dependent electron liquid correlation energies for local spin density calculations: a critical analysis. Can. J. Phys. 1980, 58, 1200-1211.

[62] Perdew, J. P.; Yue, W. Accurate and simple density functional for the electronic exchange energy: Generalized gradient approximation. Phys. Rev. B 1986, 33, 8800.

[63] Perdew, J.; Burke, K.; Ernzerhof, M. Generalized Gradient Approximation Made Simple. Phys. Rev. Lett. 1996, 77, 3865-3868.

[64] Perdew, J. P.; Ruzsinszky, A.; Csonka, G. I.; Vydrov, O. A.; Scuseria, G. E.; Constantin, L. A.; Zhou, X.; Burke, K. Restoring the density-gradient expansion for exchange in solids and surfaces. Phys. Rev. Lett. 2008, 100, 136406.

[65] Csonka, G. I.; Perdew, J. P.; Ruzsinszky, A.; Philipsen, P. H.; Lebègue, S.; Paier, J.; Vydrov, O. A.; Ángyán, J. G. Assessing the performance of recent density functionals for bulk solids. Phys. Rev. B 2009, 79, 155107.

[66] Allen, J. P.; Scanlon, D. O; Watson, G. W. Electronic structure of mixed-valence silver oxide AgO from hybrid density-functional theory. Phys. Rev. B 2010, 81, 161103.

[67] Godby, R.; Schlüter, M.; Sham, L. Accurate exchange-correlation potential for silicon and its discontinuity on addition of an electron. Phys. Rev. Lett. 1986, 56, 2415.

[68] Adamo, C.; Barone, V. Toward reliable density functional methods without adjustable parameters: The PBE0 model. J. Chem. Phys. 1999, 110, 6158.

[69] Perdew, J. P.; Ernzerhof, M.; Burke, K. Rationale for mixing exact exchange with density functional approximations. J. Chem. Phys. 1996, 105, 9982-9985.

[70] Heyd, J.; Scuseria, G. E.; Ernzerhof, M. Hybrid functionals based on a screened Coulomb potential. J. Chem. Phys. 2003, 118, 8207-8215.

[71] Heyd, J.; Scuseria, G. E.; Ernzerhof, M. Erratum: "Hybrid functionals based on a screened Coulomb potential"[J. Chem. Phys. 118, 8207 (2003)]. J. Chem. Phys. 2006, 124, 219906. 
[72] Heyd, J.; Scuseria, G. E. Efficient hybrid density functional calculations in solids: Assessment of the Heyd-Scuseria-Ernzerhof screened Coulomb hybrid functional. J. Chem. Phys. 2004, 121, 1187-1192.

[73] Grimme, S. Density functional theory with London dispersion corrections. WIREs Comput. Mol. Sci. 2011, 1, 211-228.

[74] Grimme, S. Semiempirical GGA-type density functional constructed with a long-range dispersion correction. J. Comput. Chem. 2006, 27, 1787-1799.

[75] Tkatchenko, A.; Scheffler, M. Accurate molecular van der Waals interactions from groundstate electron density and free-atom reference data. Phys. Rev. Lett. 2009, 102, 073005.

[76] Grimme, S.; Antony, J.; Ehrlich, S.; Krieg, H. A consistent and accurate ab initio parametrization of density functional dispersion correction (DFT-D) for the 94 elements H-Pu. J. Chem. Phys. 2010, 132, 154104.

[77] Tkatchenko, A.; DiStasio Jr, R. A.; Car, R.; Scheffler, M. Accurate and efficient method for many-body van der Waals interactions. Phys. Rev. Lett. 2012, 108, 236402.

[78] Ambrosetti, A.; Reilly, A. M.; DiStasio Jr, R. A.; Tkatchenko, A. Long-range correlation energy calculated from coupled atomic response functions. J. Chem. Phys. 2014, 140, 18A508.

[79] Wimmer, E.; Freeman, A.; Weinert, M.; Krakauer, H.; Hiskes, J.; Karo, A. Cesiation of W (001): Work function lowering by multiple dipole formation. Phys. Rev. Lett. 1982, 48, 1128.

[80] King-Smith, R.; Vanderbilt, D. Theory of polarization of crystalline solids. Phys. Rev. B 1993, 47, 1651.

[81] Blöchl, P. E.; Jepsen, O.; Andersen, O. K. Improved tetrahedron method for Brillouin-zone integrations. Phys. Rev. B 1994, 49, 16223.

[82] Lejaeghere, K.; Van Speybroeck, V.; Van Oost, G.; Cottenier, S. Error estimates for solidstate density-functional theory predictions: an overview by means of the ground-state elemental crystals. Crit. Rev. Solid State Mater. Sci. 2014, 39, 1-24.

[83] Feynman, R. P. Forces in molecules. Phys. Rev. 1939, 56, 340.

[84] Pulay, P. Convergence acceleration of iterative sequences. The case of SCF iteration. Chem. Phys. Lett. 1980, 73, 393-398. 
[85] Pulay, P. Ab initio calculation of force constants and equilibrium geometries in polyatomic molecules: I. Theory. Mol. Phys. 1969, 17, 197-204.

[86] https://github.com/SMTG-UCL/sumo, Accessed: 2018-03-14.

[87] Bradley, C.; Cracknell, A. P. The Mathematical Theory of Symmetry in Solids: Representation Theory for Points Groups and Space Groups; Clarendon Press, 1972.

[88] Yang, Z.; Chueh, C.-C.; Zuo, F.; Kim, J. H.; Liang, P.-W.; Jen, A. K.-Y. High-Performance Fully Printable Perovskite Solar Cells via Blade-Coating Technique under the Ambient Condition. Adv. Energy Mater. 2015, 5, 1500328.

[89] Walsh, A.; Butler, K. T. Prediction of Electron Energies in Metal Oxides. Acc. Chem. Res. 2013, 47, 364-372.

[90] Burton, L. A.; Walsh, A. Band Alignment in SnS Thin-Film Solar Cells: Possible Origin of the Low Conversion Efficiency. Appl. Phys. Lett. 2013, 102, 132111.

[91] https://github.com/WMD-group/MacroDensity, Accessed: 2018-03-14.

[92] Dronskowski, R.; Blöchl, P. E. Crystal Orbital Hamilton Populations (COHP): EnergyResolved Visualization of Chemical Bonding in Solids Based on Density-Functional Calculations. J. Phys. Chem. 1993, 97, 8617-8624.

[93] Deringer, V. L.; Tchougréeff, A. L.; Dronskowski, R. Crystal orbital Hamilton population (COHP) analysis as projected from plane-wave basis sets. J. Phys. Chem. A 2011, 115, 54615466.

[94] Sanchez-Portal, D.; Artacho, E.; Soler, J. M. Projection of plane-wave calculations into atomic orbitals. Solid State Commun. 1995, 95, 685-690.

[95] Maintz, S.; Deringer, V. L.; Tchougréeff, A. L.; Dronskowski, R. Analytic Projection from Plane-Wave and Paw Wavefunctions and Application to Chemical-Bonding Analysis in Solids. J. Comput. Chem. 2013, 34, 2557-2567.

[96] Maintz, S.; Deringer, V. L.; Tchougréeff, A. L.; Dronskowski, R. LOBSTER: A tool to extract chemical bonding from plane-wave based DFT. J. Comput. Chem. 2016, 37, 1030-1035.

[97] Jepsen, O.; Andersen, O. No error in the tetrahedron integration scheme. Phys. Rev. B 1984, 29, 5965. 
[98] Bunge, C. F.; Barrientos, J. A.; Bunge, A. V. Roothaan-Hartree-Fock ground-state atomic wave functions: Slater-type orbital expansions and expectation values for $\mathrm{Z}=2-54$. At. Data Nucl. Data Tables 1993, 53, 113-162.

[99] Koga, T.; Kanayama, K.; Watanabe, S.; Thakkar, A. J. Analytical Hartree-Fock wave functions subject to cusp and asymptotic constraints: $\mathrm{He}$ to $\mathrm{Xe}, \mathrm{Li}^{+}$to $\mathrm{Cs}^{+}, \mathrm{H}^{-}$to $\mathrm{I}^{-}$. Int. J. Quantum Chem. 1999, 71, 491-497.

[100] Gajdoš, M.; Hummer, K.; Kresse, G.; Furthmüller, J.; Bechstedt, F. Linear optical properties in the projector-augmented wave methodology. Phys. Rev. B 2006, 73, 045112.

[101] Sham, L.; Rice, T. Many-particle derivation of the effective-mass equation for the Wannier exciton. Phys. Rev. 1966, 144, 708.

[102] Birkett, M.; Savory, C. N.; Fioretti, A. N.; Thompson, P.; Muryn, C. A.; Weerakkody, A.; Mitrovic, I.; Hall, S.; Treharne, R.; Dhanak, V. R.; Scanlon, D.; Zakutayev, A.; TD, V. Atypically small temperature-dependence of the direct band gap in the metastable semiconductor copper nitride $\mathrm{Cu}_{3}$ N. Phys. Rev. B 2017, 95, 115201.

[103] Baroni, S.; Resta, R. Ab initio calculation of the macroscopic dielectric constant in silicon. Phys. Rev. B 1986, 33, 7017.

[104] Gonze, X.; Lee, C. Dynamical matrices, Born effective charges, dielectric permittivity tensors, and interatomic force constants from density-functional perturbation theory. Phys. Rev. B 1997, 55, 10355.

[105] Nunes, R.; Gonze, X. Berry-phase treatment of the homogeneous electric field perturbation in insulators. Phys. Rev. B 2001, 63, 155107.

[106] Lany, S.; Zunger, A. Assessment of correction methods for the band-gap problem and for finite-size effects in supercell defect calculations: Case studies for $\mathrm{ZnO}$ and GaAs. Phys. Rev. B 2008, 78, 235104.

[107] Freysoldt, C.; Neugebauer, J.; Van de Walle, C. G. Fully ab initio finite-size corrections for charged-defect supercell calculations. Phys. Rev. Lett. 2009, 102, 016402.

[108] Makov, G.; Payne, M. Periodic boundary conditions in ab initio calculations. Phys. Rev. B 1995, 51, 4014. 
[109] Lany, S.; Zunger, A. Accurate prediction of defect properties in density functional supercell calculations. Modell. Simul. Mater. Sci. Eng. 2009, 17, 084002.

[110] Murphy, S. T.; Hine, N. D. Anisotropic charge screening and supercell size convergence of defect formation energies. Phys. Rev. B 2013, 87, 094111.

[111] Buckeridge, J.; Scanlon, D.; Walsh, A.; Catlow, C. R. A. Automated procedure to determine the thermodynamic stability of a material and the range of chemical potentials necessary for its formation relative to competing phases and compounds. Comput. Phys. Commun. 2014, 185, 330-338.

[112] Kittel, C.; Kroemer, H.; Scott, H. Thermal physics 2nd ed. Freeman 1980, Chap. 13.

[113] Taylor, F. H.; Buckeridge, J.; Catlow, C. R. A. Defects and oxide ion migration in the solid oxide fuel cell cathode material $\mathrm{LaFeO}_{3}$. Chem. Mater. 2016, 28, 8210-8220.

[114] Buckeridge, J.; Jevdokimovs, D.; Catlow, C.; Sokol, A. Nonstoichiometry and Weyl fermionic behavior in TaAs. Phys. Rev. B 2016, 94, 180101.

[115] https://github.com/jbuckeridge/sc-fermi, Accessed: 2018-03-14.

[116] Shockley, W.; Read Jr, W. Statistics of the recombinations of holes and electrons. Phys. Rev. 1952, 87, 835.

[117] Kresse, G.; Hafner, J. Ab initio molecular dynamics for liquid metals. Phys. Rev. B 1993, 47, 558-561.

[118] Kresse, G.; Hafner, J. Ab initio molecular-dynamics simulation of the liquid-metal amorphous-semiconductor transition in germanium. Phys. Rev. B 1994, 49, 14251-14269.

[119] Kresse, G.; Furthmüller, J. Efficient iterative schemes for ab initio total-energy calculations using a plane-wave basis set. Phys. Rev. B 1996, 54, 11169-11186.

[120] Kresse, G.; Furthmüller, J. Efficiency of ab initio total energy calculations for metals and semiconductors using a plane wave basis set. Comput. Mater. Sci. 1996, 6, 15.

[121] Kresse, G.; Joubert, D. From ultrasoft pseudopotentials to the projector augmented-wave method. Phys. Rev. B 1999, 59, 1758.

[122] Momma, K.; Izumi, F. VESTA: a three-dimensional visualization system for electronic and structural analysis. J. Appl. Crystallogr. 2008, 41, 653-658. 
[123] Kim, H.-S.; Park, N.-G. Parameters Affecting I-V Hysteresis of $\mathrm{CH}_{3} \mathrm{NH}_{3} \mathrm{PbI}_{3}$ Perovskite Solar Cells: Effects of Perovskite Crystal Size and Mesoporous $\mathrm{TiO}_{2}$ Layer. J. Phys. Chem. Lett. 2014, 5, 2927-2934.

[124] Beilsten-Edmands, J.; Eperon, G. E.; Johnson, R. D.; Snaith, H. J.; Radaelli, P. G. Nonferroelectric nature of the conductance hysteresis in $\mathrm{CH}_{3} \mathrm{NH}_{3} \mathrm{PbI}_{3}$ perovskite-based photovoltaic devices. Appl. Phys. Lett. 2015, 106, 173502.

[125] Frost, J. M.; Walsh, A. What is moving in hybrid halide perovskite solar cells? Acc. Chem. Res. 2016, 49, 528-535.

[126] Azpiroz, J. M.; Mosconi, E.; Bisquert, J.; De Angelis, F. Defect migration in methylammonium lead iodide and its role in perovskite solar cell operation. Energy Environ. Sci. 2015, $8,2118-2127$.

[127] Eames, C.; Frost, J. M.; Barnes, P. R.; O’regan, B. C.; Walsh, A.; Islam, M. S. Ionic transport in hybrid lead iodide perovskite solar cells. Nat. Commun. 2015, 6, 7497.

[128] Tidhar, Y.; Edri, E.; Weissman, H.; Zohar, D.; Hodes, G.; Cahen, D.; Rybtchinski, B.; Kirmayer, S. Crystallization of methyl ammonium lead halide perovskites: implications for photovoltaic applications. J. Am. Chem. Soc. 2014, 136, 13249-56.

[129] Jeon, N. J.; Noh, J. H.; Kim, Y. C.; Yang, W. S.; Ryu, S.; Seok, S. I. Solvent engineering for high-performance inorganic-organic hybrid perovskite solar cells. Nat. Mater. 2014, 13, 897-903.

[130] Leo, K. Perovskite photovoltaics: Signs of stability. Nat. Nanotechnol. 2015, 10, 574-575.

[131] Hailegnaw, B.; Kirmayer, S.; Edri, E.; Hodes, G.; Cahen, D. Rain on Methyl-AmmoniumLead-Iodide Based Perovskites: Possible Environmental Effects of Perovskite Solar Cells. J. Phys. Chem. Lett. 2015, 6, 1543-1547.

[132] Bass, K. K.; McAnally, R. E.; Zhou, S.; Djurovich, P. I.; Thompson, M. E.; Melot, B. C. Influence of moisture on the preparation, crystal structure, and photophysical properties of organohalide perovskites. Chem. Commun. 2014, 50, 15819-15822.

[133] Mosconi, E.; Azpiroz, J. M.; De Angelis, F. Ab Initio Molecular Dynamics Simulations of Methylammonium Lead Iodide Perovskite Degradation by Water. Chem. Mater. 2015, 27, 4885-4892. 
[134] Christians, J. A.; Manser, J. S.; Kamat, P. V. Multifaceted Excited State of $\mathrm{CH}_{3} \mathrm{NH}_{3} \mathrm{PbI}_{3}$. Charge Separation, Recombination, and Trapping. J. Phys. Chem. Lett. 2015, 6, 2086-2095.

[135] Bryant, D.; Aristidou, N.; Pont, S.; Sanchez-Molina, I.; Chotchunangatchaval, T.; Wheeler, S.; Durrant, J. R.; Haque, S. A. Light and oxygen induced degradation limits the operational stability of methylammonium lead triiodide perovskite solar cells. Energy Environ. Sci. 2016, 9, 1655-1660.

[136] Conings, B.; Drijkoningen, J.; Gauquelin, N.; Babayigit, A.; D’Haen, J.; D’Olieslaeger, L.; Ethirajan, A.; Verbeeck, J.; Manca, J.; Mosconi, E.; Angelis, F. D.; Boyen, H.-G. Intrinsic Thermal Instability of Methylammonium Lead Trihalide Perovskite. Adv. Energy Mater. $2015,5,1500477$.

[137] Juarez-Perez, E. J.; Hawash, Z.; Raga, S. R.; Ono, L. K.; Qi, Y. Thermal degradation of $\mathrm{CH}_{3} \mathrm{NH}_{3} \mathrm{PbI}_{3}$ perovskite into $\mathrm{NH}_{3}$ and $\mathrm{CH}_{3} \mathrm{I}$ gases observed by coupled thermogravimetry-mass spectrometry analysis. Energy Environ. Sci. 2016, 9, 3406-3410.

[138] Niu, G.; Li, W.; Meng, F.; Wang, L.; Dong, H.; Qiu, Y. Study on the Stability of $\mathrm{CH}_{3} \mathrm{NH}_{3} \mathrm{PbI}_{3}$ Films and the Effect of Post-Modification by Aluminum Oxide in All-Solid-State Hybrid Solar Cells. J. Mater. Chem. A 2014, 2, 705-710.

[139] Pisoni, A.; Jaćimović, J.; Barišić, O. S.; Spina, M.; Gaál, R.; Forró, L.; Horváth, E. Ultra-Low Thermal Conductivity in Organic-Inorganic Hybrid Perovskite $\mathrm{CH}_{3} \mathrm{NH}_{3} \mathrm{PbI}_{3}$. J. Phys. Chem. Lett. 2014, 5, 2488-2492.

[140] Zhang, Y.-Y.; Chen, S.; Xu, P.; Xiang, H.; Gong, X.-G.; Walsh, A.; Wei, S.-H. Intrinsic instability of the hybrid halide perovskite semiconductor $\mathrm{CH}_{3} \mathrm{NH}_{3} \mathrm{PbI}_{3}$. Chinese Physics Letters 2018, 35, 036104.

[141] Ganose, A. M.; Savory, C. N.; Scanlon, D. O. $\left(\mathrm{CH}_{3} \mathrm{NH}_{3}\right)_{2} \mathrm{~Pb}(\mathrm{SCN})_{2} \mathrm{I}_{2}$ : A More Stable Structural Motif for Hybrid Halide Photovoltaics? J. Phys. Chem. Lett. 2015, 6, 4594-4598.

[142] Habisreutinger, S. N.; McMeekin, D. P.; Snaith, H. J.; Nicholas, R. J. Research Update: Strategies for Improving the Stability of Perovskite Solar Cells. APL Mater. 2016, 4, 091503.

[143] Mitzi, D. B. Templating and Structural Engineering in Organic-Inorganic Perovskites. J. Chem. Soc., Dalton Trans. 2001, 1-12.

[144] Boix, P. P.; Agarwala, S.; Koh, T. M.; Mathews, N.; Mhaisalkar, S. G. Perovskite Solar Cells: Beyond Methylammonium Lead Iodide. J. Phys. Chem. Lett. 2015, 6, 898-907. 
[145] Saparov, B.; Mitzi, D. B. Organic-Inorganic Perovskites: Structural Versatility for Functional Materials Design. Chem. Rev. 2016, 116, 4558-4596.

[146] Amat, A.; Mosconi, E.; Ronca, E.; Quarti, C.; Umari, P.; Nazeeruddin, M. K.; Grätzel, M.; De Angelis, F. Cation-induced band-gap tuning in organohalide perovskites: Interplay of spin-orbit coupling and octahedra tilting. Nano Lett. 2014, 14, 3608-3616.

[147] Eperon, G. E.; Stranks, S. D.; Menelaou, C.; Johnston, M. B.; Herz, L. M.; Snaith, H. J. Formamidinium lead trihalide: a broadly tunable perovskite for efficient planar heterojunction solar cells. Energy Environ. Sci. 2014, 7, 982-988.

[148] Stoumpos, C. C.; Malliakas, C. D.; Kanatzidis, M. G. Semiconducting tin and lead iodide perovskites with organic cations: Phase transitions, high mobilities, and near-infrared photoluminescent properties. Inorg. Chem. 2013, 52, 9019-9038.

[149] Weller, M. T.; Weber, O. J.; Frost, J. M.; Walsh, A. Cubic Perovskite Structure of Black Formamidinium Lead Iodide, $\alpha$-[HC( $\left.\left(\mathrm{NH}_{2}\right)_{2}\right] \mathrm{PbI}_{3}$, at 298 K. J. Phys. Chem. Lett. 2015, 6, 3209-3212.

[150] Binek, A.; Hanusch, F. C.; Docampo, P.; Bein, T. Stabilization of the trigonal hightemperature phase of formamidinium lead iodide. J. Phys. Chem. Lett. 2015, 6, 1249-1253.

[151] Pellet, N.; Gao, P.; Gregori, G.; Yang, T.-Y.; Nazeeruddin, M. K.; Maier, J.; Grätzel, M. Mixed-Organic-Cation Perovskite Photovoltaics for Enhanced Solar-Light Harvesting. Angew. Chem. Int. Ed. 2014, 53, 3151-3157.

[152] Brgoch, J.; Lehner, A.; Chabinyc, M.; Seshadri, R. Ab Initio Calculations of Band Gaps and Absolute Band Positions of Polymorphs of $\mathrm{RbPbI}_{3}$ and $\mathrm{CsPbI}_{3}$ : Implications for MainGroup Halide Perovskite Photovoltaics. J. Phys. Chem. C 2014, 118, 27721-27727.

[153] Sutton, R. J.; Eperon, G. E.; Miranda, L.; Parrott, E. S.; Kamino, B. A.; Patel, J. B.; Hörantner, M. T.; Johnston, M. B.; Haghighirad, A. A.; Moore, D. T.; Snaith, H. J. BandgapTunable Cesium Lead Halide Perovskites with High Thermal Stability for Efficient Solar Cells. Adv. Energy Mater. 2016, 6, 1502458.

[154] Eperon, G. E.; Paterno, G. M.; Sutton, R. J.; Zampetti, A.; Haghighirad, A. A.; Cacialli, F.; Snaith, H. J. Inorganic caesium lead iodide perovskite solar cells. J. Mater. Chem. A 2015, 3, 19688-19695. 
[155] Protesescu, L.; Yakunin, S.; Bodnarchuk, M. I.; Krieg, F.; Caputo, R.; Hendon, C. H.; Yang, R. X.; Walsh, A.; Kovalenko, M. V. Nanocrystals of cesium lead halide perovskites $(\mathrm{CsPbX}, \mathrm{X}=\mathrm{Cl}, \mathrm{Br}$, and $\mathrm{I})$ : novel optoelectronic materials showing bright emission with wide color gamut. Nano letters 2015, 15, 3692-3696.

[156] Stranks, S. D.; Eperon, G. E.; Grancini, G.; Menelaou, C.; Alcocer, M. J. P.; Leijtens, T.; Herz, L. M.; Petrozza, A.; Snaith, H. J. Electron-Hole Diffusion Lengths Exceeding 1 Micrometer in an Organometal Trihalide Perovskite Absorber. Science 2013, 342, 341-344.

[157] Yantara, N.; Fang, Y.; Chen, S.; Dewi, H. A.; Boix, P. P.; Mhaisalkar, S. G.; Mathews, N. Unravelling the Effects of $\mathrm{Cl}$ Addition in Single Step $\mathrm{CH}_{3} \mathrm{NH}_{3} \mathrm{PbI}_{3}$ Perovskite Solar Cells. Chem. Mater, 2015, 27, 2309-2314.

[158] Noh, J. H.; Im, S. H.; Heo, J. H.; Mandal, T. N.; Seok, S. I. Chemical Management for Colorful, Efficient, and Stable Inorganic-Organic Hybrid Nanostructured Solar Cells. Nano Lett. 2013, 13, 1764-1769.

[159] Rehman, W.; Milot, R. L.; Eperon, G. E.; Wehrenfennig, C.; Boland, J. L.; Snaith, H. J.; Johnston, M. B.; Herz, L. M. Charge-Carrier Dynamics and Mobilities in Formamidinium Lead Mixed-Halide Perovskites. Adv. Mater. 2015, 27, 7938-7944.

[160] Brivio, F.; Caetano, C.; Walsh, A. Thermodynamic Origin of Photoinstability in the $\mathrm{CH}_{3} \mathrm{NH}_{3} \mathrm{~Pb}\left(\mathrm{I}_{1-x} \mathrm{Br}_{x}\right)_{3}$ Hybrid Halide Perovskite Alloy. J. Phys. Chem. Lett. 2016, 7, 10831087.

[161] McMeekin, D. P.; Sadoughi, G.; Rehman, W.; Eperon, G. E.; Saliba, M.; Hörantner, M. T.; Haghighirad, A.; Sakai, N.; Korte, L.; Rech, B.; Johnston, M. B.; Herz, L. M.; Snaith, H. J. A mixed-cation lead mixed-halide perovskite absorber for tandem solar cells. Science 2016, 351, 151-155.

[162] Duong, T. et al. Rubidium Multication Perovskite with Optimized Bandgap for PerovskiteSilicon Tandem with over 26\% Efficiency. Adv. Energy Mater. 2017, 7, 1700228.

[163] Koh, T. M.; Thirumal, K.; Soo, H. S.; Mathews, N. Multidimensional Perovskites: A Mixed Cation Approach Towards Ambient Stable and Tunable Perovskite Photovoltaics. ChemSusChem 2016, 9, 2541-2558. 
[164] Ganose, A. M.; Savory, C. N.; Scanlon, D. O. Beyond methylammonium lead iodide: prospects for the emergent field of $n s^{2}$ containing solar absorbers. Chem. Commun. 2017, $53,20-44$.

[165] Gregor, K.; Shijing, S.; Anthony, K. C. Solid-State Principles Applied to Organic-Inorganic Perovskites: New Tricks for an Old Dog. Chem. Sci. 2014, 5, 4712-4715.

[166] Kieslich, G.; Sun, S.; Cheetham, T. An Extended Tolerance Factor Approach for OrganicInorganic Perovskites. Chem. Sci. 2015, 6, 3430-3433.

[167] Mitzi, D. B.; Dimitrakopoulos, C. D.; Kosbar, L. L. Structurally Tailored OrganicInorganic Perovskites: Optical Properties and Solution-Processed Channel Materials for Thin-Film Transistors. Chem. Mater. 2001, 13, 3728-3740.

[168] Mitzi, D. B.; Chondroudis, K.; Kagan, C. R. Organic-inorganic electronics. IBM J. Res. Dev. 2001, 45, 29-45.

[169] Mitzi, D. B.; Medeiros, D. R.; Malenfant, P. R. L. Intercalated Organic-Inorganic Perovskites Stabilized by Fluoroaryl - Aryl Interactions. Inorg. Chem. 2002, 41, 2134-2145.

[170] Quan, L. N.; Yuan, M.; Comin, R.; Voznyy, O.; Beauregard, E. M.; Hoogland, S.; Buin, A.; Kirmani, A. R.; Zhao, K.; Amassian, A.; Kim, D. H.; Sargent, E. H. Ligand-Stabilized Reduced-Dimensionality Perovskites. J. Am. Chem. Soc. 2016, 138, 2649-2655.

[171] Jiang, W.; Ying, J.; Zhou, W.; Shen, K.; Liu, X.; Gao, X.; Guo, F.; Gao, Y.; Yang, T. A New Layered Nano Hybrid Perovskite Film with Enhanced Resistance to Moisture-Induced Degradation. Chem. Phys. Lett. 2016, 658, 71-75.

[172] Mitzi, D. B.; Feild, C. A.; Harrison, W. T. A.; Guloy, A. M. Conducting Tin Halides with a Layered Organic-Based Perovskite Structure. Nature 1994, 369, 467-469.

[173] Mitzi, D. B. In Progress in Inorganic Chemistry, Volume 48; Karlin, K. D., Ed.; John Wiley \& Sons Inc., 1999; Chapter 1, pp 1-121.

[174] Wang, S.; Mitzi, D. B.; Feild, C. A.; Guloys, A. Synthesis and Characterization of $\left[\mathrm{NH}_{2} \mathrm{C}(\mathrm{I})=\mathrm{NH}_{2}\right]_{3} \mathrm{MI}_{5}(\mathrm{M}=\mathrm{Sn}, \mathrm{Pb})$ : Stereochemical Activity in Divalent Tin and Lead Halides Containing Single (110) Perovskite Sheets. J. Am. Chem. Soc. 1995, 117, 5297-5302.

[175] Mitzi, D. B. Synthesis, Crystal Structure, and Optical and Thermal Properties of $\left(\mathrm{C}_{4} \mathrm{H}_{9} \mathrm{NH}_{3}\right)_{2} \mathrm{MI}_{4}(\mathrm{M}=\mathrm{Ge}, \mathrm{Sn}, \mathrm{Pb})$. Chem. Mater. 1996, 8, 791-800. 
[176] Chondroudis, K.; Mitzi, D. B. Electroluminescence from an Organic-Inorganic Perovskite Incorporating a Quaterthiophene Dye within Lead Halide Perovskite Layers. Chem. Mater. 1999, 11, 3028-3030.

[177] Mitzi, D. B. Organic-Inorganic Perovskites Containing Trivalent Metal Halide Layers: The Templating Influence of the Organic Cation Layer. Inorg. Chem. 2000, 39, 6107-6113.

[178] Mitzi, D. B. Thin-Film Deposition of Organic-Inorganic Hybrid Materials. Chem. Mater. 2001, 13, 3283-3298.

[179] Kitazawa, N.; Watanabe, Y. Optical properties of natural quantum-well compounds $\left(\mathrm{C}_{6} \mathrm{H}_{5}-\mathrm{C}_{n} \mathrm{H}_{2 n}-\mathrm{NH}_{3}\right)_{2} \mathrm{PbBr}_{4}(\mathrm{n}=1-4)$. J. Phys. Chem. Solids 2010, 71, 797-802.

[180] Ishihara, T.; Takahashi, J.; Goto, T. Optical properties due to electronic transitions in twodimensional semiconductors $\left(\mathrm{C}_{n} \mathrm{H}_{2 n+1} \mathrm{NH}_{3}\right)_{2} \mathrm{PbI}_{4}$. Phys. Rev. B 1990, 42, 11099-11107.

[181] Muljarov, E. A.; Tikhodeev, S. G.; Gippius, N. A.; Ishihara, T. Excitons in self-organized semiconductor/insulator superlattices: PbI-based perovskite compounds. Phys. Rev. B 1995, 51, 14370-14378.

[182] Smith, I. C.; Hoke, E. T.; Solis-Ibarra, D.; McGehee, M. D.; Karunadasa, H. I. A Layered Hybrid Perovskite Solar-Cell Absorber with Enhanced Moisture Stability. Angew. Chem. Int. Ed. 2014, 53, 11232-11235.

[183] Cao, D. H.; Stoumpos, C. C.; Farha, O. K.; Hupp, J. T.; Kanatzidis, M. G. 2D Homologous Perovskites as Light-Absorbing Materials for Solar Cell Applications. J. Am. Chem. Soc. 2015, 137, 7843-7850.

[184] Dualeh, A.; Gao, P.; Seok, S. I.; Nazeeruddin, M. K.; Grätzel, M. Thermal Behavior of Methylammonium Lead-Trihalide Perovskite Photovoltaic Light Harvesters. Chem. Mater. 2014, 26, 6160-6164.

[185] Christians, J. A.; Herrera, P. A. M.; Kamat, P. V. Transformation of the Excited State and Photovoltaic Efficiency Cells $\mathrm{NH}_{3} \mathrm{PbI}_{3}$ Perovskite upon Controlled Exposure to Humidified Air. J. Am. Chem. Soc. 2015, 137, 1530-1538.

[186] Directive 2011/65/EU of the European Parliament and of the Council of 8 June 2011 on the restriction of the use of certain hazardous substances in electrical and electronic equipment (recast). http://eur-lex. europa.eu/legal-content/en/TXT/?uri=celex : 32011L0065, 2011. 
[187] Winship, K. Toxicity of tin and its compounds. Adverse Drug React. Acute. Poisoning Rev. 1987, 7, 19-38.

[188] Umari, P.; Mosconi, E.; De Angelis, F. Relativistic GW calculations on $\mathrm{CH}_{3} \mathrm{NH}_{3} \mathrm{PbI}_{3}$ and $\mathrm{CH}_{3} \mathrm{NH}_{3} \mathrm{SnI}_{3}$ perovskites for solar cell applications. Sci. Rep. 2014, 4, 4467.

[189] Brenner, T. M.; Egger, D. A.; Kronik, L.; Hodes, G.; Cahen, D. Hybrid organic-inorganic perovskites: low-cost semiconductors with intriguing charge-transport properties. Nat. Rev. Mater. 2016, 1, 15007.

[190] Koh, T. M.; Krishnamoorthy, T.; Yantara, N.; Shi, C.; Leong, W. L.; Boix, P. P.; Grimsdale, A. C.; Mhaisalkar, S. G.; Mathews, N. Formamidinium tin-based perovskite with low $E_{\mathrm{g}}$ for photovoltaic applications. J. Mater. Chem. A 2015, 3, 14996-15000.

[191] Koh, T. M.; Fu, K.; Fang, Y.; Chen, S.; Sum, T.; Mathews, N.; Mhaisalkar, S. G.; Boix, P. P.; Baikie, T. Formamidinium-containing metal-halide: an alternative material for near-IR absorption perovskite solar cells. J. Mater. Chem. C 2013, 118, 16458-16462.

[192] Lee, S. J.; Shin, S. S.; Kim, Y. C.; Kim, D.; Ahn, T. K.; Noh, J. H.; Seo, J.; Seok, S. I. Fabrication of Efficient Formamidinium Tin Iodide Perovskite Solar Cells through $\mathrm{SnF}_{2}-$ Pyrazine Complex. J. Am. Chem. Soc. 2016, 138, 3974-3977.

[193] Shao, S.; Liu, J.; Portale, G.; Fang, H.-H.; Blake, G. R.; ten Brink, G. H.; Koster, L. J. A.; Loi, M. A. Highly Reproducible Sn-Based Hybrid Perovskite Solar Cells with 9\% Efficiency. Adv. Energy Mater. 2018, 8, 1702019.

[194] Chung, I.; Lee, B.; He, J.; Chang, R. P.; Kanatzidis, M. G. All-solid-state dye-sensitized solar cells with high efficiency. Nature 2012, 485, 486-489.

[195] Chung, I.; Song, J.-H.; Im, J.; Androulakis, J.; Malliakas, C. D.; Li, H.; Freeman, A. J.; Kenney, J. T.; Kanatzidis, M. G. CsSnI 3 : semiconductor or metal? High electrical conductivity and strong near-infrared photoluminescence from a single material. High hole mobility and phase-transitions. J. Am. Chem. Soc. 2012, 134, 8579-8587.

[196] Zhou, Y.; Garces, H. F.; Senturk, B. S.; Ortiz, A. L.; Padture, N. P. Room temperature "one-pot" solution synthesis of nanoscale $\mathrm{CsSnI}_{3}$ orthorhombic perovskite thin films and particles. Mater. Lett. 2013, 110, 127-129.

[197] Chen, Z.; Wang, J. J.; Ren, Y.; Yu, C.; Shum, K. Schottky solar cells based on CsSnI 3 thinfilms. Appl. Phys. Lett. 2012, 101, 093901. 
[198] Sabba, D.; Mulmudi, H. K.; Prabhakar, R. R.; Krishnamoorthy, T.; Baikie, T.; Boix, P. P.; Mhaisalkar, S.; Mathews, N. Impact of Anionic $\mathrm{Br}^{-}$Substitution on Open Circuit Voltage in Lead Free Perovskite (CsSnI ${ }_{3-x} \mathrm{Br}_{x}$ ) Solar Cells. J. Phys. Chem. C 2015, 119, 1763-1767.

[199] Kumar, M. H.; Dharani, S.; Leong, W. L.; Boix, P. P.; Prabhakar, R. R.; Baikie, T.; Shi, C.; Ding, H.; Ramesh, R.; Asta, M.; Graetzel, M.; Mhaisalkar, S. G.; Mathews, N. Lead-free halide perovskite solar cells with high photocurrents realized through vacancy modulation. Adv. Mater. 2014, 26, 7122-7127.

[200] Dharani, S.; Mulmudi, H. K.; Yantara, N.; Trang, P. T. T.; Park, N. G.; Graetzel, M.; Mhaisalkar, S.; Mathews, N.; Boix, P. P. High efficiency electrospun $\mathrm{TiO}_{2}$ nanofiber based hybrid organic-inorganic perovskite solar cell. Nanoscale 2014, 6, 1675-1679.

[201] Kumar, M. H.; Yantara, N.; Dharani, S.; Graetzel, M.; Mhaisalkar, S.; Boix, P. P.; Mathews, N. Flexible, low-temperature, solution processed $\mathrm{ZnO}$-based perovskite solid state solar cells. Chem. Commun. 2013, 49, 11089-11091.

[202] Yu, C.; Ren, Y.; Chen, Z.; Shum, K. First-principles study of structural phase transitions in $\mathrm{CsSnI}_{3}$. J. Appl. Phys. 2013, 114, 163505.

[203] da Silva, E. L.; Skelton, J. M.; Parker, S. C.; Walsh, A.; Silva, E. L.; Skelton, J. M.; Parker, S. C.; Walsh, A. Phase stability and transformations in the halide perovskite $\mathrm{CsSnI}_{3}$. Phys. Rev. B 2015, 91, 1-12.

[204] Cao, D. H.; Stoumpos, C. C.; Yokoyama, T.; Logsdon, J. L.; Song, T.-B.; Farha, O. K.; Wasielewski, M. R.; Hupp, J. T.; Kanatzidis, M. G. Thin Films and Solar Cells Based on Semiconducting Two-Dimensional Ruddlesden-Popper $\left(\mathrm{CH}_{3}\left(\mathrm{CH}_{2}\right)_{3} \mathrm{NH}_{3}\right)_{2}\left(\mathrm{CH}_{3} \mathrm{NH}_{3}\right)_{n-1} \mathrm{Sn}_{n} \mathrm{I}_{3 n+1}$ Perovskites. ACS Energy Lett. 2017, 2, 982-990.

[205] Liao, Y.; Liu, H.; Zhou, W.; Yang, D.; Shang, Y.; Shi, Z.; Li, B.; Jiang, X.; Zhang, L.; Quan, L. N.; Quintero-Bermudez, R.; Sutherland, B. R.; Mi, Q.; Sargent, E. H.; Ning, Z. Highly Oriented Low-Dimensional Tin Halide Perovskites with Enhanced Stability and Photovoltaic Performance. J. Am. Chem. Soc. 2017, 139, 6693-6699.

[206] Chen, C.; Li, W.; Zhou, Y.; Chen, C.; Luo, M.; Liu, X.; Zeng, K.; Yang, B.; Zhang, C.; Han, J.; Tang, J. Optical properties of amorphous and polycrystalline $\mathrm{Sb}_{2} \mathrm{Se}_{3}$ thin films prepared by thermal evaporation. Appl. Phys. Lett. 2015, 107, 043905. 
[207] Halder, A.; Chulliyil, R.; Subbiah, A. S.; Khan, T.; Chattoraj, S.; Chowdhury, A.; Sarkar, S. K. Pseudohalide ( $\left.\mathrm{SCN}^{-}\right)$-Doped $\mathrm{MAPbI}_{3}$ Perovskites: A Few Surprises. J. Phys. Chem. Lett. 2015, 6, 3483-3489.

[208] Jiang, Q.; Rebollar, D.; Gong, J.; Piacentino, E. L.; Zheng, C.; Xu, T. Pseudohalide-Induced Moisture Tolerance in Perovskite $\mathrm{CH}_{3} \mathrm{NH}_{3} \mathrm{~Pb}(\mathrm{SCN})_{2} \mathrm{I}$ Thin Films. Angew. Chem. Int. Ed. $2015,54,7617-7620$.

[209] Daub, M.; Hillebrecht, H. Synthesis, Single-Crystal Structure and Characterization of $\left(\mathrm{CH}_{3} \mathrm{NH}_{3}\right)_{2} \mathrm{~Pb}(\mathrm{SCN})_{2} \mathrm{I}_{2}$. Angew. Chem. 2015, 127, 11168-11169.

[210] Iwadate, Y.; Kawamura, K.; Igarashi, K.; Mochinaga, J. Effective ionic radii of nitrite and thiocyanate estimated in terms of the Boettcher equation and the Lorentz-Lorenz equation. J. Phys. Chem. 1982, 86, 5205-5208.

[211] Snaith, H. J.; Abate, A.; Ball, J. M.; Eperon, G. E.; Leijtens, T.; Noel, N. K.; Stranks, S. D.; Wang, J. T.-W.; Wojciechowski, K.; Zhang, W. Anomalous Hysteresis in Perovskite Solar Cells. J. Phys. Chem. Lett. 2014, 5, 1511-1515.

[212] Chen, Y.; Li, B.; Huang, W.; Gao, D.; Liang, Z. Efficient and reproducible $\mathrm{CH}_{3} \mathrm{NH}_{3} \mathrm{PbI}_{3-x}(\mathrm{SCN})_{x}$ perovskite based planar solar cells. Chem. Commun. 2015, 51, 11997-11999.

[213] Xiao, Z.; Meng, W.; Saparov, B.; Duan, H.-S.; Wang, C.; Feng, C.; Liao, W.-Q.; Ke, W.; Zhao, D.; Wang, J.; Mitzi, D. B.; Yan, Y. Photovoltaic Properties of Two-Dimensional $\left(\mathrm{CH}_{3} \mathrm{NH}_{3}\right)_{2} \mathrm{~Pb}(\mathrm{SCN})_{2} \mathrm{I}_{2}$ Perovskite: A Combined Experimental and Density-Functional Theory Study. J. Phys. Chem. Lett. 2016, 7, 1213-1218.

[214] Umeyama, D.; Lin, Y.; Karunadasa, H. I. Red-to-Black Piezochromism in a Compressible $\mathrm{Pb}-\mathrm{I}-\mathrm{SCN}$ Layered Perovskite. Chem. Mater. 2016, 28, 3241-3244.

[215] Younts, R.; Duan, H.-s.; Gautam, B.; Saparov, B.; Liu, J.; Mongin, C.; Castellano, F. N.; Mitzi, D. B.; Gundogdu, K. Efficient Generation of Long-Lived Triplet Excitons in 2D Hybrid Perovskite. Adv. Mater. 2017, 1-7.

[216] Tanaka, K.; Kondo, T. Bandgap and exciton binding energies in lead-iodide-based natural quantum-well crystals. Sci. Technol. Adv. Mater. 2003, 4, 599-604.

[217] Wei, S.-H.; Zunger, A. Calculated natural band offsets of all II-VI and III-V semiconductors: Chemical trends and the role of cation d orbitals. Appl. Phys. Lett. 1998, 72, 2011-2013. 
[218] Wei, S.-H.; Zunger, A. Role of Metal d States in II-VI Semiconductors. Phys. Rev. B 1988, 37, 8958.

[219] Williams, D. J.; Daemen, L.; Vogel, S.; Proffen, T. Temperature Dependence of the Crystal Structure of $\alpha$-AgSCN by Powder Neutron Diffraction. J. Appl. Crystallogr. 2007, 40, 10391043.

[220] Hendon, C. H.; Yang, R. X.; Burton, L. A.; Walsh, A. Assessment of Polyanion (BF- And $\mathrm{PF}_{6}^{-}$) Substitutions in Hybrid Halide Perovskites. J. Mater. Chem. A 2015, 3, 9067-9070.

[221] Frost, J. M.; Butler, K. T.; Walsh, A. Molecular Ferroelectric Contributions to Anomalous Hysteresis in Hybrid Perovskite Solar Cells. APL Mater. 2014, 2, 081506.

[222] Fernandes, P.; Salomé, P.; Da Cunha, A. Precursors' Order Effect on the Properties of Sulfurized $\mathrm{Cu}_{2} \mathrm{ZnSnS}_{4}$ Thin Films. Semicond. Sci. Technol. 2009, 24, 105013.

[223] Mosconi, E.; Amat, A.; Nazeeruddin, M. K.; Grätzel, M.; De Angelis, F. First-Principles Modeling of Mixed Halide Organometal Perovskites for Photovoltaic Applications. J. Mater. Chem. C 2013, 117, 13902-13913.

[224] Butler, K. T.; Frost, J. M.; Walsh, A. Ferroelectric Materials for Solar Energy Conversion: Photoferroics Revisited. Energy Environ. Sci. 2015, 8, 838-848.

[225] Burschka, J.; Pellet, N.; Moon, S.-J.; Humphry-Baker, R.; Gao, P.; Nazeeruddin, M. K.; Grätzel, M. Sequential Deposition as a Route to High-Performance Perovskite-Sensitized Solar Cells. Nature 2013, 499, 316-319.

[226] Xiao, Z.; Meng, W.; Wang, J.; Yan, Y. Defect properties of the two-dimensional $\left(\mathrm{CH}_{3} \mathrm{NH}_{3}\right)_{2} \mathrm{~Pb}(\mathrm{SCN})_{2} \mathrm{I}_{2}$ perovskite: a density-functional theory study. Phys. Chem. Chem. Phys. 2016, 18, 25786-25790.

[227] Payne, D. J.; Egdell, R. G.; Walsh, A.; Watson, G. W.; Guo, J.; Glans, P. A.; Learmonth, T.; Smith, K. E. Electronic Origins of Structural Distortions in Post-Transition Metal Oxides: Experimental and Theoretical Evidence for a Revision of the Lone Pair Model. Phys. Rev. Lett. 2006, 96, 157403.

[228] Walsh, A.; Payne, D. J.; Egdell, R. G.; Watson, G. W. Stereochemistry of post-transition metal oxides: revision of the classical lone pair model. Chem. Soc. Rev. 2011, 40, 44554463. 
[229] others, et al. CRC handbook of chemistry and physics; CRC press, Boca raton FL, 1989; Vol. 1990.

[230] James, A. M.; Lord, M. P. Macmillan's chemical and physical data; Macmillan London, 1992.

[231] Liu, J.; Shi, J.; Li, D.; Zhang, F.; Li, X.; Xiao, Y.; Wang, S. Molecular design and photovoltaic performance of a novel thiocyanate-based layered organometal perovskite material. Synth. Met. 2016, 215, 56-63.

[232] Helander, M.; Greiner, M.; Wang, Z.; Tang, W.; Lu, Z. Work Function of Fluorine Doped Tin Oxide. J. Vac. Sci. Technol. A 2011, 29, 011019.

[233] https://github.com/SMTG-UCL/MAPSI, Accessed: 2014-09-27.

[234] Hao, F.; Stoumpos, C. C.; Cao, D. H.; Chang, R. P. H.; Kanatzidis, M. G. Lead-free solidstate organic-inorganic halide perovskite solar cells. Nature Photon. 2014, 8, 489-494.

[235] Noel, N. K.; Stranks, S. D.; Abate, A.; Wehrenfennig, C.; Guarnera, S.; Haghighirad, A.; Sadhanala, A.; Eperon, G. E.; Pathak, S. K.; Johnston, M. B.; Petrozza, A.; Herz, L.; Snaith, H. Lead-Free Organic-Inorganic Tin Halide Perovskites for Photovoltaic Applications. Energy Environ. Sci. 2014, 7, 3061-3068.

[236] Walsh, A. Principles of Chemical Bonding and Band Gap Engineering in Hybrid OrganicInorganic Halide Perovskites. J. Mater. Chem. C 2015, 119, 5755-5760.

[237] Anderson, M. T.; Greenwood, K. B.; Taylor, G. A.; Poeppelmeier, K. R. B-cation arrangements in double perovskites. Prog. Solid State Chem. 1993, 22, 197-233.

[238] McClure, E. T.; Ball, M. R.; Windl, W.; Woodward, P. M. $\mathrm{Cs}_{2} \operatorname{AgBiX}_{6}(\mathrm{X}=\mathrm{Br}, \mathrm{Cl})$ : new visible light absorbing, lead-free halide perovskite semiconductors. Chem. Mater. 2016, $28,1348-1354$.

[239] Slavney, A. H.; Hu, T.; Lindenberg, A. M.; Karunadasa, H. I. A bismuth-halide double perovskite with long carrier recombination lifetime for photovoltaic applications. J. Am. Chem. Soc. 2016, 138, 2138-2141.

[240] Savory, C. N.; Walsh, A.; Scanlon, D. O. Can Pb-free halide double perovskites support high-efficiency solar cells? ACS Energy Lett. 2016, 1, 949-955. 
[241] Lee, B.; Stoumpos, C. C.; Zhou, N.; Hao, F.; Malliakas, C.; Yeh, C.-Y.; Marks, T. J.; Kanatzidis, M. G.; Chang, R. P. Air-stable molecular semiconducting iodosalts for solar cell applications: $\mathrm{Cs}_{2} \mathrm{SnI}_{6}$ as a hole conductor. J. Am. Chem. Soc. 2014, 136, 15379-15385.

[242] Saparov, B.; Sun, J.-P.; Meng, W.; Xiao, Z.; Duan, H.-S.; Gunawan, O.; Shin, D.; Hill, I. G.; Yan, Y.; Mitzi, D. B. Thin-film deposition and characterization of a Sn-deficient perovskite derivative $\mathrm{Cs}_{2} \mathrm{SnI}_{6}$. Chem. Mater. 2016, 28, 2315-2322.

[243] Jiang, Y.; Zhang, H.; Qiu, X.; Cao, B. The air and thermal stabilities of lead-free perovskite variant $\mathrm{Cs}_{2} \mathrm{SnI}_{6}$ powder. Mater. Lett. 2017, 199, 50-52.

[244] Xiao, Z.; Zhou, Y.; Hosono, H.; Kamiya, T. Intrinsic defects in a photovoltaic perovskite variant $\mathrm{Cs}_{2} \mathrm{SnI}_{6}$. Phys. Chem. Chem. Phys. 2015, 17, 18900-18903.

[245] Kaltzoglou, A.; Antoniadou, M.; Kontos, A. G.; Stoumpos, C. C.; Perganti, D.; Siranidi, E.; Raptis, V.; Trohidou, K.; Psycharis, V.; Kanatzidis, M. G.; Falaras, P. Optical-vibrational properties of the $\mathrm{Cs}_{2} \mathrm{SnX}_{6}(\mathrm{X}=\mathrm{Cl}, \mathrm{Br}, \mathrm{I})$ defect perovskites and hole-transport efficiency in dye-sensitized solar cells. J. Phys. Chem. C 2016, 120, 11777-11785.

[246] Qiu, X.; Cao, B.; Yuan, S.; Chen, X.; Qiu, Z.; Jiang, Y.; Ye, Q.; Wang, H.; Zeng, H.; Liu, J.; Kanatzidis, M. From unstable $\mathrm{CsSnI}_{3}$ to air-stable $\mathrm{Cs}_{2} \mathrm{SnI}_{6}$ : A lead-free perovskite solar cell light absorber with bandgap of $1.48 \mathrm{eV}$ and high absorption coefficient. Sol. Energy Mater. Sol. Cells 2017, 159, 227-234.

[247] Qiu, X.; Jiang, Y.; Zhang, H.; Qiu, Z.; Yuan, S.; Wang, P.; Cao, B. Lead-free mesoscopic $\mathrm{Cs}_{2} \mathrm{SnI}_{6}$ perovskite solar cells using different nanostructured $\mathrm{ZnO}$ nanorods as electron transport layers. Phys. Status Solidi RRL 2016, 10, 587-591.

[248] Lee, B.; Krenselewski, A.; Baik, S. I.; Seidman, D. N.; Chang, R. P. Solution processing of air-stable molecular semiconducting iodosalts, $\mathrm{Cs}_{2} \mathrm{SnI}_{6-x} \mathrm{Br}_{x}$, for potential solar cell applications. Sustain. Energy Fuels 2017, 1, 710-724.

[249] Dolzhnikov, D. S.; Wang, C.; Xu, Y.; Kanatzidis, M. G.; Weiss, E. A. Ligand-Free, QuantumConfined $\mathrm{Cs}_{2} \mathrm{SnI}_{6}$ Perovskite Nanocrystals. Chem. Mater. 2017, 29, 7901-7907.

[250] Xiao, Z.; Hosono, H.; Kamiya, T. Origin of Carrier Generation in Photovoltaic Perovskite Variant $\mathrm{Cs}_{2} \mathrm{SnI}_{6}$. Bull. Chem. Soc. Jpn. 2015, 88, 1250-1255.

[251] https://github.com/SMTG-UCL/CSI-CTI, Accessed: 2018-03-14. 
[252] Zhang, J.; Yu, C.; Wang, L.; Li, Y.; Ren, Y.; Shum, K. Energy barrier at the N719-dye/CsSnI 3 interface for photogenerated holes in dye-sensitized solar cells. Sci. Rep. 2014, 4, 6954.

[253] Peresh, E. Y.; Zubaka, O.; Sidei, V.; Barchii, I.; Kun, S.; Kun, A. Preparation, stability regions, and properties of $\mathrm{M}_{2} \mathrm{TeI}_{6}(\mathrm{M}=\mathrm{Rb}, \mathrm{Cs}, \mathrm{Tl})$ crystals. Inorg. Mater. 2002, 38, 859-863.

[254] Ranfagni, A.; Mugnai, D.; Bacci, M.; Viliani, G.; Fontana, M. The optical properties of thallium-like impurities in alkali-halide crystals. Adv. Phys. 1983, 32, 823-905.

[255] Blasse, G.; Dirksen, G.; Abriel, W. The influence of distortion of the Te (IV) coordination octahedron on its luminescence. Chem. Phys. Lett. 1987, 136, 460-464.

[256] Drummen, P.; Donker, H.; Smit, W.; Blasse, G. Jahn-Teller distortion in the excited state of tellurium (IV) in $\mathrm{Cs}_{2} \mathrm{MCl}_{6}(\mathrm{M}=\mathrm{Zr}, \mathrm{Sn})$. Chem. Phys. Lett. 1988, 144, 460-462.

[257] Donker, H.; Smit, W.; Blasse, G. On the luminescence of $\mathrm{Te}_{4+}$ in $\mathrm{A}_{2} \mathrm{ZrCl}_{6}(\mathrm{~A}=\mathrm{Cs}, \mathrm{Rb})$ and $\mathrm{A}_{2} \mathrm{ZrCl}_{6}(\mathrm{~A}=\mathrm{Cs}, \mathrm{Rb}, \mathrm{K})$. J. Phys. Chem. Solids 1989, 50, 603-609.

[258] Dalpian, G. M.; Liu, Q.; Stoumpos, C. C.; Douvalis, A. P.; Balasubramanian, M.; Kanatzidis, M. G.; Zunger, A. Changes in charge density vs changes in formal oxidation states: The case of Sn halide perovskites and their ordered vacancy analogues. Phys. Rev. Mater. 2017, 1, 025401.

[259] Raebiger, H.; Lany, S.; Zunger, A. Charge self-regulation upon changing the oxidation state of transition metals in insulators. Nature 2008, 453, 763.

[260] Walsh, A.; Da Silva, J. L.; Wei, S.-H.; Körber, C.; Klein, A.; Piper, L.; DeMasi, A.; Smith, K. E.; Panaccione, G.; Torelli, P. Nature of the band gap of $\operatorname{In}_{2} \mathrm{O}_{3}$ revealed by firstprinciples calculations and X-ray spectroscopy. Phys. Rev. Lett. 2008, 100, 167402.

[261] Godinho, K. G.; Carey, J. J.; Morgan, B. J.; Scanlon, D. O.; Watson, G. W. Understanding conductivity in $\mathrm{SrCu}_{2} \mathrm{O}_{2}$ : stability, geometry and electronic structure of intrinsic defects from first principles. J. Mater. Chem. 2010, 20, 1086-1096.

[262] Persson, C.; Zhao, Y.-J.; Lany, S.; Zunger, A. n-type doping of CuInSe ${ }_{2}$ and $\mathrm{CuGaSe}_{2}$. Phys. Rev. B 2005, 72, 035211.

[263] Scanlon, D. O. Defect engineering of $\mathrm{BaSnO}_{3}$ for high-performance transparent conducting oxide applications. Phys. Rev. B 2013, 87, 161201. 
[264] Xu, P.; Chen, S.; Xiang, H.-J.; Gong, X.-G.; Wei, S.-H. Influence of defects and synthesis conditions on the photovoltaic performance of perovskite semiconductor $\mathrm{CsSnI}_{3}$. Chem. Mater. 2014, 26, 6068-6072.

[265] Lehner, A. J.; Fabini, D. H.; Evans, H. A.; Hébert, C.-A.; Smock, S. R.; Hu, J.; Wang, H.; Zwanziger, J. W.; Chabinyc, M. L.; Seshadri, R. Crystal and Electronic Structures of Complex Bismuth Iodides $\mathrm{A}_{3} \mathrm{Bi}_{2} \mathrm{I}_{9}(\mathrm{~A}=\mathrm{K}, \mathrm{Rb}, \mathrm{Cs})$ Related to Perovskite: Aiding the Rational Design of Photovoltaics. Chem. Mater, 2015, 27, 7137-7148.

[266] Brandt, R. E.; Kurchin, R. C.; Hoye, R. L. Z.; Poindexter, J. R.; Wilson, M. W. B.; Sulekar, S.; Lenahan, F.; Yen, P. X. T.; Stevanović, V.; Nino, J. C.; Bawendi, M. G.; Buonassisi, T. Investigation of Bismuth Triiodide $\left(\mathrm{BiI}_{3}\right)$ for Photovoltaic Applications. J. Phys. Chem. Lett. 2015, 6, 4297-4302.

[267] Wu, L. M.; Wu, X. T.; Chen, L. Structural Overview and Structure-Property Relationships of Iodoplumbate and Iodobismuthate. Coord. Chem. Rev. 2009, 253, 2787-2804.

[268] Demartin, F.; Gramaccioli, C.; Campostrini, I. Demicheleite-(I), BiSI, a New Mineral from La Fossa Crater, Vulcano, Aeolian Islands, Italy. Mineral. Mag. 2010, 74, 141-145.

[269] Frit, B.; Mercurio, J. The Crystal Chemistry and Dielectric Properties of the Aurivillius Family of Complex Bismuth Oxides with Perovskite-Like Layered Structures. J. Alloys Compd. 1992, 188, 27-35.

[270] Brivio, F.; Butler, K. T.; Walsh, A.; Van Schilfgaarde, M. Relativistic Quasiparticle SelfConsistent Electronic Structure of Hybrid Halide Perovskite Photovoltaic Absorbers. Phys. Rev. B 2014, 89, 155204.

[271] Slikkerveer, A.; de Wolff, F. A. Pharmacokinetics and Toxicity of Bismuth Compounds. Med. Toxicol. Adverse Drug Exp. 1989, 4, 303-323.

[272] Serfontein, W.; Mekel, R. Bismuth Toxicity in Man II. Review of Bismuth Blood and Urine Levels in Patients After Administration of Therapeutic Bismuth Formulations in Relation to the Problem of Bismuth Toxicity in Man. Res. Commun. Chem. Pathol. Pharmacol. 1979, 26, 391-411.

[273] Leonard, N. M.; Wieland, L. C.; Mohan, R. S. Applications of bismuth(III) compounds in organic synthesis. Tetrahedron 2002, 58, 8373 - 8397. 
[274] Mohan, R. Green bismuth. Nat. Chem. 2010, 2, 336-336.

[275] Fabini, D. H.; Labram, J. G.; Lehner, A. J.; Bechtel, J. S.; Evans, H. A.; Van der Ven, A.; Wudl, F.; Chabinyc, M. L.; Seshadri, R. Main-group halide semiconductors derived from perovskite: distinguishing chemical, structural, and electronic aspects. Inorg. Chem. 2016, $56,11-25$.

[276] Moreno-García, H.; Nair, M. T. S.; Nair, P. K. Chemically deposited lead sulfide and bismuth sulfide thin films and $\mathrm{Bi}_{2} \mathrm{~S}_{3} / \mathrm{PbS}$ solar cells. Thin Solid Films 2011, 519, 2287 - 2295.

[277] ten Haaf, S.; Sträter, H.; Brüggemann, R.; Bauer, G. H.; Felser, C.; Jakob, G. Physical vapor deposition of $\mathrm{Bi}_{2} \mathrm{~S}_{3}$ as absorber material in thin film photovoltaics. Thin Solid Films 2013, $535,394-397$.

[278] Filip, M. R.; Patrick, C. E.; Giustino, F. GW quasiparticle band structures of stibnite, antimonselite, bismuthinite, and guanajuatite. Phys. Rev. B 2013, 87, 205125.

[279] Pramanik, P.; Bhattacharya, R. N. A Chemical Method for Deposition of Thin Film of $\mathrm{Bi}_{2} \mathrm{~S}_{3}$. J. Electrochem. Soc. 1980, 127, 2087.

[280] Bhattacharya, R. N.; Pramanik, P. Semiconductor Liquid Junction Solar Cell Based on Chemically Deposited $\mathrm{Bi}_{2} \mathrm{~S}_{3}$ Thin Film and Some Semiconducting Properties of Bismuth Chalcogenides. J. Electrochem. Soc. 1982, 129, 332-335.

[281] Calixto-Rodriguez, M.; García, H. M.; Nair, M. T. S.; Nair, P. K. Antimony Chalcogenide/Lead Selenide Thin Film Solar Cell with 2.5ECS J. Solid State Sci. Technol. 2013, 2, Q69-Q73.

[282] Tumelero, M. A.; Faccio, R.; Pasa, A. A. Unraveling the Native Conduction of Trichalcogenides and Its Ideal Band Alignment for New Photovoltaic Interfaces. J. Phys. Chem. C 2016, 120, 1390-1399.

[283] Rath, A. K.; Bernechea, M.; Martinez, L.; Konstantatos, G. Solution-Processed Heterojunction Solar Cells Based on $p$-type PbS Quantum Dots and $n$-type $\mathrm{Bi}_{2} \mathrm{~S}_{3}$ Nanocrystals. Adv. Mater. 2011, 23, 3712-3717.

[284] Rath, A. K.; Bernechea, M.; Martinez, L.; de Arquer, F. P. G.; Osmond, J.; Konstantatos, G. Solution-processed inorganic bulk nano-heterojunctions and their application to solar cells. Nat. Photon. 2012, 6, 529-534. 
[285] Martinez, L.; Bernechea, M.; de Arquer, F. P. G.; Konstantatos, G. Near IR-Sensitive, Nontoxic, Polymer/Nanocrystal Solar Cells Employing $\mathrm{Bi}_{2} \mathrm{~S}_{3}$ as the Electron Acceptor. Adv. Energy Mater. 2011, 1, 1029-1035.

[286] Martinez, L.; Stavrinadis, A.; Higuchi, S.; Diedenhofen, S. L.; Bernechea, M.; Tajima, K.; Konstantatos, G. Hybrid solution-processed bulk heterojunction solar cells based on bismuth sulfide nanocrystals. Phys. Chem. Chem. Phys. 2013, 15, 5482-5487.

[287] Whittaker-Brooks, L.; Gao, J.; Hailey, A. K.; Thomas, C. R.; Yao, N.; Loo, Y.-L. $\mathrm{Bi}_{2} \mathrm{~S}_{3}$ nanowire networks as electron acceptor layers in solution-processed hybrid solar cells. J. Mater. Chem. C 2015, 3, 2686-2692.

[288] Han, D.; Du, M.-H.; Dai, C.-M.; Sun, D.; Chen, S. Influence of defects and dopants on the photovoltaic performance of $\mathrm{Bi}_{2} \mathrm{~S}_{3}$ : first-principles insights. J. Mater. Chem.A 2017, 5, 6200-6210.

[289] Ruck, M. Darstellung und Kristallstruktur von fehlordnungfreiem Bismuttriiodid. Z. Kristallogr. 1995, 210, 650-655.

[290] Podraza, N. J.; Qiu, W.; Hinojosa, B. B.; Xu, H.; Motyka, M. A.; Phillpot, S. R.; Baciak, J. E.; Trolier-McKinstry, S.; Nino, J. C. Band gap and structure of single crystal $\mathrm{BiI}_{3}$ : Resolving discrepancies in literature. J. Appl. Phys. 2013, 114, 033110.

[291] Yorikawa, H.; Muramatsu, S. Theoretical study of crystal and electronic structures of $\mathrm{BiI}_{3}$. J. Phys. Condens. Matter 2008, 20, 325220.

[292] Lehner, A. J.; Wang, H.; Fabini, D. H.; Liman, C. D.; Hébert, C.-A.; Perry, E. E.; Wang, M.; Bazan, G. C.; Chabinyc, M. L.; Seshadri, R. Electronic structure and photovoltaic application of $\mathrm{BiI}_{3}$. Appl. Phys. Lett. 2015, 107, 131109.

[293] Hamdeh, U. H.; Nelson, R. D.; Ryan, B. J.; Bhattacharjee, U.; Petrich, J. W.; Panthani, M. G. Solution-processed $\mathrm{BiI}_{3}$ thin films for photovoltaic applications: Improved carrier collection via solvent annealing. Chem. Mater. 2016, 28, 6567-6574.

[294] Cho, S. B.; Gazquez, J.; Huang, X.; Myung, Y.; Banerjee, P.; Mishra, R. Intrinsic point defects and intergrowths in layered bismuth triiodide. Phys. Rev. Mater. 2018, 2, 064602.

[295] Geller, S.; Wernick, J. H. Ternary Semiconducting Compounds with Sodium ChlorideLike Structure: AgSbSe ${ }_{2}, \mathrm{AgSbTe}_{2}, \mathrm{AgBiS}_{2}, \mathrm{AgBiSe}_{2}$. Acta Crystallogr. 1959, 12, 46-54. 
[296] Huang, P.-C.; Yang, W.-C.; Lee, M.-W. AgBiS 2 Semiconductor-Sensitized Solar Cells. J. Phys. Chem. C 2013, 117, 18308-18314.

[297] Pejova, B.; Grozdanov, I.; Nesheva, D.; Petrova, A. Size-Dependent Properties of Sonochemically Synthesized Three-Dimensional Arrays of Close-Packed Semiconducting $\mathrm{AgBiS}_{2}$ Quantum Dots. Chem. Mater. 2008, 20, 2551-2565.

[298] Pejova, B.; Nesheva, D.; Aneva, Z.; Petrova, A. Photoconductivity and Relaxation Dynamics in Sonochemically Synthesized Assemblies of $\mathrm{AgBiS}_{2}$ Quantum Dots. J. Phys. Chem. C 2011, 115, 37-46.

[299] Chen, C.; Qiu, X.; Ji, S.; Jia, C.; Ye, C. The synthesis of monodispersed AgBiS 2 quantum dots with a giant dielectric constant. CrystEngComm 2013, 15, 7644-7648.

[300] Zhou, S.; Yang, J.; Li, W.; Jiang, Q.; Luo, Y.; Zhang, D.; Zhou, Z.; Li, X. Preparation and Photovoltaic Properties of Ternary AgBiS 2 Quantum Dots Sensitized $\mathrm{TiO}_{2}$ Nanorods Photoanodes by Electrochemical Atomic Layer Deposition. J. Electrochem. Soc. 2016, 163, D63-D67.

[301] Bernechea, M.; Miller, N. C.; Xercavins, G.; So, D.; Stavrinadis, A.; Konstantatos, G. Solution-processed solar cells based on environmentally friendly $\mathrm{AgBiS}_{2}$ nanocrystals. Nat. Photon. 2016, 10, 521-525.

[302] Fourcroy, P.; Palazzi, M.; Rivet, J.; Flahaut, J.; Céolin, R. Etude du systeme AgIBiI 3 . Mater. Res. Bull. 1979, 14, 325 - 328.

[303] Kim, Y.; Yang, Z.; Jain, A.; Voznyy, O.; Kim, G.-H.; Liu, M.; Quan, L. N.; García de Arquer, F. P.; Comin, R.; Fan, J. Z.; Sargent, E. H. Pure Cubic-Phase Hybrid Iodobismuthates $\mathrm{AgBi}_{7} \mathrm{I}_{7}$ for Thin-Film Photovoltaics. Angew. Chem. Int. Ed. 2016, 55, 9586.

[304] Xiao, Z.; Meng, W.; Mitzi, D. B.; Yan, Y. Crystal structure of $\mathrm{AgBi}_{2} \mathrm{I}_{7}$ thin films. J. Phys. Chem. Lett. 2016, 7, 3903-3907.

[305] Turkevych, I.; Kazaoui, S.; Ito, E.; Urano, T.; Yamada, K.; Tomiyasu, H.; Yamagishi, H.; Kondo, M.; Aramaki, S. Photovoltaic Rudorffites: Lead-Free Silver Bismuth Halides Alternative to Hybrid Lead Halide Perovskites. ChemSusChem 2017, 10, 3754-3759.

[306] Saparov, B.; Hong, F.; Sun, J.-P.; Duan, H.-S.; Meng, W.; Cameron, S.; Hill, I. G.; Yan, Y.; Mitzi, D. B. Thin-Film Preparation and Characterization of $\mathrm{Cs}_{3} \mathrm{Sb}_{2} \mathrm{I}_{9}$ : A Lead-Free Layered Perovskite Semiconductor. Chem. Mater. 2015, 27, 5622-5632. 
[307] Lindquist, O. Crystal Structure of Caesium Bismuth Iodide $\mathrm{Cs}_{3} \mathrm{Bi}_{2} \mathrm{I}_{9}$. Acta Chem. Scand. 1968, 22, 2943-2952.

[308] Chabot, B.; Parthé, E. $\mathrm{Cs}_{3} \mathrm{Sb}_{2} \mathrm{I}_{9}$ and $\mathrm{Cs}_{3} \mathrm{Bi}_{2} \mathrm{I}_{9}$ with the hexagonal $\mathrm{Cs}_{3} \mathrm{Cr}_{2} \mathrm{Cl}_{9}$ structure type. Acta Crystallogr., Sect. B: Struct. Sci. 1978, 34, 645-648.

[309] Eckhardt, K.; Bon, V.; Getzschmann, J.; Grothe, J.; Wisser, F. M.; Kaskel, S. Crystallographic insights into $\left(\mathrm{CH}_{3} \mathrm{NH}_{3}\right)_{3}\left(\mathrm{Bi}_{2} \mathrm{I}_{9}\right)$ : a new lead-free hybrid organic-inorganic material as a potential absorber for photovoltaics. Chem. Commun. 2016, 52, 3058-3060.

[310] Hoye, R. L. Z.; Brandt, R. E.; Osherov, A.; Stevanović, V.; Stranks, S. D.; Wilson, M. W. B.; Kim, H.; Akey, A. J.; Perkins, J. D.; Kurchin, R. C.; Poindexter, J. R.; Wang, E. N.; Bawendi, M. G.; Bulović, V.; Buonassisi, T. Methylammonium Bismuth Iodide as a LeadFree, Stable Hybrid Organic-Inorganic Solar Absorber. Chem. Eur. J. 2016, 22, 2605-2610.

[311] Kawai, T.; Shimanuki, S. Optical Studies of $\left(\mathrm{CH}_{3} \mathrm{NH}_{3}\right)_{3} \mathrm{Bi}_{2} \mathrm{I}_{9}$ Single Crystals. Phys. Status Solidi B 1993, 177, K43-K45.

[312] Park, B.-W.; Philippe, B.; Zhang, X.; Rensmo, H.; Boschloo, G.; Johansson, E. M. J. Bismuth Based Hybrid Perovskites $\mathrm{A}_{3} \mathrm{Bi}_{2} \mathrm{I}_{9}$ (A: Methylammonium or Cesium) for Solar Cell Application. Adv. Mater. 2015, 27, 6806-6813.

[313] Lyu, M.; Yun, J.-H.; Cai, M.; Jiao, Y.; Bernhardt, P. V.; Zhang, M.; Wang, Q.; Du, A.; Wang, H.; Liu, G.; Wang, L. Organic-inorganic bismuth (III)-based material: A lead-free, air-stable and solution-processable light-absorber beyond organolead perovskites. Nano Res. 2016, 9, 692-702.

[314] Öz, S.; Hebig, J.-C.; Jung, E.; Singh, T.; Lepcha, A.; Olthof, S.; Jan, F.; Gao, Y.; German, R.; van Loosdrecht, P. H.; Meerholz, K.; Kirchartz, T.; Mathur, S. Zero-dimensional $\left(\mathrm{CH}_{3} \mathrm{NH}_{3}\right)_{3} \mathrm{Bi}_{2} \mathrm{I}_{9}$ perovskite for optoelectronic applications. Sol. Energy Mater. Sol. Cells 2016, 158, 195-201.

[315] Fabian, D. M.; Ardo, S. Hybrid organic-inorganic solar cells based on bismuth iodide and 1,6-hexanediammonium dication. J. Mater. Chem. A 2016, 4, 6837-6841.

[316] Braun, T. P.; DiSalvo, F. J. Bismuth Selenide Iodide. Acta Crystallogr., Sect. C: Cryst. Struct. Commun. 2000, 56, 1-2. 
[317] Zhou, Y.; Wang, L.; Chen, S.; Qin, S.; Liu, X.; Chen, J.; Xue, D.-J.; Luo, M.; Cao, Y.; Cheng, Y. Thin-Film $\mathrm{Sb}_{2} \mathrm{Se}_{3}$ Photovoltaics with Oriented One-Dimensional Ribbons and Benign Grain Boundaries. Nat. Photonics 2015, 9, 409-415.

[318] Hahn, N. T.; Rettie, A. J.; Beal, S. K.; Fullon, R. R.; Mullins, C. B. n-BiSI Thin Films: Selenium Doping and Solar Cell Behavior. J. Mater. Chem. C 2012, 116, 24878-24886.

[319] Hahn, N. T.; Self, J. L.; Mullins, C. B. BiSI Micro-Rod Thin Films: Efficient Solar Absorber Electrodes? J. Phys. Chem. Lett. 2012, 3, 1571-1576.

[320] Kunioku, H.; Higashi, M.; Abe, R. Low-Temperature Synthesis of Bismuth Chalcohalides: Candidate Photovoltaic Materials with Easily, Continuously Controllable Band gap. Sci. Rep. 2016, 6, 32664.

[321] https://github.com/SMTG-UCL/BiChI, Accessed: 2015-11-12.

[322] Chepur, D.; Bercha, D.; Turyanitsa, I.; Slivka, V. Y. Peculiarities of the Energy Spectrum and Edge Absorption in the Chain Compounds $\mathrm{A}^{\mathrm{V}} \mathrm{B}^{\mathrm{VI}} \mathrm{C}^{\mathrm{VII}}$. Phys. Status Solidi B 1968, 30, 461-468.

[323] Shin, D.-W.; Hyun, S.-C.; Park, S.-a.; Kim, Y.-G.; Kim, C.-d.; Kim, W.-T. Optical properties of undoped and Ni-doped VA-VI $\mathrm{V}^{\mathrm{A}}-\mathrm{VII}{ }^{\mathrm{A}}$ single crystals. J. Phys. Chem. Solids 1994, 55, 825-830.

[324] Park, S.-A.; Kim, M.-Y.; Lim, J.-Y.; Park, B.-S.; Koh, J.-D.; Kim, W.-T. Optical Properties of Undoped and V-Doped VA-VI ${ }^{\mathrm{A}}-$ VII ${ }^{\mathrm{A}}$ Single Crystals. Phys. Status Solidi B 1995, 187, 253-260.

[325] Hyun, S.-C.; Kim, Y.-G.; Kim, M.-Y.; Koh, J.-D.; Park, B.-S.; Kim, W.-T. Optical Properties of Undoped and Chromium-DopedVA-VI ${ }^{\mathrm{A}}-\mathrm{VII}^{\mathrm{A}}$ Single Crystals. J. Mater. Sci. 1995, 30, 6113-6117.

[326] Dunitz, J.; Orgel, L. Stereochemistry of Ionic Solids. Adv. Inorg. Chem. Radiochem. 1960, $2,1-60$.

[327] Orgel, L. 769. The Stereochemistry of B Subgroup Metals. Part II. The Inert Pair. J. Chem. Soc. 1959, 3815-3819.

[328] Walsh, A.; Watson, G. W. The Origin of the Stereochemically Active Pb (II) Lone Pair: DFT Calculations on PbO and PbS. J. Solid State Chem. 2005, 178, 1422-1428. 
[329] Temple, D. J.; Kehoe, A. B.; Allen, J. P.; Watson, G. W.; Scanlon, D. O. Geometry, Electronic Structure, and Bonding in $\mathrm{CuMCh}_{2}(\mathrm{M}=\mathrm{Sb}, \mathrm{Bi} ; \mathrm{Ch}=\mathrm{S}$, Se): Alternative Solar Cell Absorber Materials? J. Mater. Chem. C 2012, 116, 7334-7340.

[330] Zhang, S.; Wei, S.-H.; Zunger, A.; Katayama-Yoshida, H. Defect Physics of the CuInSe 2 Chalcopyrite Semiconductor. Phys. Rev. B 1998, 57, 9642.

[331] Walsh, A.; Watson, G. W. Influence of the Anion On Lone Pair Formation in Sn (II) Monochalcogenides: A DFT Study. J. Phys. Chem. B 2005, 109, 18868-18875.

[332] Walsh, A.; Watson, G. W.; Payne, D. J.; Edgell, R. G.; Guo, J.; Glans, P.-A.; Learmonth, T.; Smith, K. E. Electronic Structure of the $\alpha$ and $\delta$ Phases of $\mathrm{Bi}_{2} \mathrm{O}_{3}$ : A Combined Ab Initio and X-ray Spectroscopy Study. Phys. Rev. B 2006, 73, 235104.

[333] Waghmare, U.; Spaldin, N.; Kandpal, H.; Seshadri, R. First-Principles Indicators of Metallicity and Cation Off-Centricity in the IV-VI Rocksalt Chalcogenides of Divalent Ge, Sn, and Pb. Phys. Rev. B 2003, 67, 125111.

[334] Walsh, A.; Watson, G. W. Polymorphism in Bismuth Stannate: A First-Principles Study. Chem. Mater. 2007, 19, 5158-5164.

[335] Svensson, C. The Crystal Structure of Orthorhombic Antimony Trioxide, $\mathrm{Sb}_{2} \mathrm{O}_{3}$. Acta Crystallogr., Sect. B: Struct. Sci 1974, 30, 458-461.

[336] Guo, D.; Hu, C.; Zhang, C. First-Principles Study on Doping and Temperature Dependence of Thermoelectric Property of $\mathrm{Bi}_{2} \mathrm{~S}_{3}$ Thermoelectric Material. Mater. Res. Bull. 2013, 48, 1984-1988.

[337] Cardona, M.; Peter, Y. Y. Fundamentals of Semiconductors; Springer, 2005.

[338] Allan, G.; Delerue, C.; Lannoo, M.; Martin, E. Hydrogenic Impurity Levels, Dielectric Constant, and Coulomb Charging Effects in Silicon Crystallites. Phys. Rev. B 1995, 52, 11982.

[339] Jaegermann, W.; Klein, A.; Mayer, T. Interface Engineering of Inorganic Thin-Film Solar Cells-Materials-Science Challenges for Advanced Physical Concepts. Adv. Mater. 2009, $21,4196-4206$. 
[340] Chen, Z.; Peng, W.; Zhang, K.; Zhang, J.; Yang, X.; Numata, Y.; Han, L. Band Alignment By Ternary Crystalline Potential-Tuning Interlayer for Efficient Electron Injection in Quantum Dot-Sensitized Solar Cells. J. Mater. Chem. A 2014, 2, 7004-7014.

[341] MacDonald, B. I.; Martucci, A.; Rubanov, S.; Watkins, S. E.; Mulvaney, P.; Jasieniak, J. J. Layer-by-Layer Assembly of Sintered CdSe ${ }_{x} \mathrm{Te}_{1-x}$ Nanocrystal Solar Cells. ACS Nano 2012, 6, 5995-6004.

[342] Chen, S.; Walsh, A.; Yang, J.-H.; Gong, X.; Sun, L.; Yang, P.-X.; Chu, J.-H.; Wei, S.-H. Compositional Dependence of Structural and Electronic Properties Of $\mathrm{Cu}_{2} \mathrm{ZnSn}(\mathrm{S}, \mathrm{Se})_{4}$ Alloys for Thin Film Solar Cells. Phys. Rev. B 2011, 83, 125201.

[343] Boix, P. P.; Larramona, G.; Jacob, A.; Delatouche, B.; Mora-Seró, I.; Bisquert, J. Hole Transport and Recombination in All-Solid $\mathrm{Sb}_{2} \mathrm{~S}_{3}$-Sensitized $\mathrm{TiO}_{2}$ Solar Cells Using CuSCN As Hole Transporter. J. Mater. Chem. C 2011, 116, 1579-1587.

[344] Chao, X.; Lei, C.; Hongchun, Y. Study on the Synthesis, Characterization of p-CuSCN/n-Si Heterojunction. Open Materials Science Journal 2013, 7, 29-32.

[345] Inbasekaran, M.; Woo, E.; Wu, W.; Bernius, M.; Wujkowski, L. Fluorene Homopolymers and Copolymers. Synth. Met. 2000, 111, 397-401.

[346] Dong, C.; Yu, W.; Xu, M.; Cao, J.; Chen, C.; Yu, W.; Wang, Y. Valence Band Offset of $\mathrm{Cu}_{2} \mathrm{O} / \mathrm{In}_{2} \mathrm{O}_{3}$ Heterojunction Determined By X-Ray Photoelectron Spectroscopy. J. Appl. Phys. 2011, 110, 073712.

[347] Höffling, B.; Schleife, A.; Rödl, C.; Bechstedt, F. Band Discontinuities at Si-TCO Interfaces from Quasiparticle Calculations: Comparison of Two Alignment Approaches. Phys. Rev. B 2012, 85, 035305.

[348] Brandt, R. E.; Poindexter, J. R.; Gorai, P.; Kurchin, R. C.; Hoye, R. L.; Nienhaus, L.; Wilson, M. W.; Polizzotti, J. A.; Sereika, R.; Žaltauskas, R.; Lee, L. C.; MacManusDriscoll, J. L.; Bawendi,; Stevanović, V.; Buonassisi, T. Searching for "defect-tolerant" photovoltaic materials: combined theoretical and experimental screening. Chem. Mater. 2017, $29,4667-4674$.

[349] Alkauskas, A.; Dreyer, C. E.; Lyons, J. L.; Van de Walle, C. G. Role of excited states in Shockley-Read-Hall recombination in wide-band-gap semiconductors. Phys. Rev. B 2016, 93, 201304. 